Julio Cesar Saldaña Pumarica

\title{
Sistemas de detecção e classificação de impulsos elétricos de sinais neurais extracelulares
}

Tese apresentada à Escola Politécnica da Universidade de São Paulo para obtenção do Título de Doutor em Ciências, Programa de Engenharia Elétrica. 
Julio Cesar Saldaña Pumarica

\title{
Sistemas de detecção e classificação de impulsos elétricos de sinais neurais extracelulares
}

\author{
Tese apresentada à Escola Politécnica \\ da Universidade de São Paulo para \\ obtenção do Título de Doutor em \\ Ciências, Programa de Engenharia \\ Elétrica.
}

Área de concentração:

Microeletrônica

Orientador:

Prof. Dr. Livre-Docente

Emilio Del Moral Hernandez 
Este exemplar foi revisado e corrigido em relação à versão original, sob responsabilidade única do autor e com a anuência de seu orientador.

São Paulo, de de

Assinatura do autor:

Assinatura do orientador:

Catalogação-na-publicação

\section{Saldana Pumarica, Julio Cesar}

Sistemas de detecção e classificação de impulsos elétricos de sinais neurais extracelulares / J. C. Saldana Pumarica -- versão corr. -- São Paulo, 2016.

$137 \mathrm{p}$.

Tese (Doutorado) - Escola Politécnica da Universidade de São Paulo. Departamento de Engenharia de Sistemas Eletrônicos.

1.microeletrônica 2.cmos I.Universidade de São Paulo. Escola Politécnica. Departamento de Engenharia de Sistemas Eletrônicos II.t. 


\section{Resumo}

O registro de sinais neurais através de matrizes de microeletrodos implantáveis no meio extracelular do córtex cerebral tem-se tornado um paradigma experimental para a neurociência. Por outro lado, a pesquisa recente sobre neuropróteses motoras tem mostrado que é possível decodificar comandos motores a partir dos sinais registrados no meio extracelular do córtex cerebral. Em ambos os contextos, neurociência experimental e desenvolvimento de neuropróteses motoras, um dos aspectos encontrados no estado da arte é a utilização de circuitos integrados (chips) implantados no cérebro. Nesses chips, os sinais neurais medidos com os microeletrodos são amplificados, filtrados, processados e transmitidos a um computador externo mediante fios que atravessam o crânio. Existe o interesse em desenvolver chips implantáveis que transmitam os sinais ao computador externo sem a necessidade de fios que atravessem o crânio. Na pesquisa do estado da arte tem-se encontrado a utilização de tais chips implantáveis sem fio em ratos e macacos, porém até a data da elaboração deste texto não foram encontrados relatos da aplicação em humanos. Um dos aspectos que deve se levar em consideração no desenvolvimento de interfaces neurais implantáveis sem fio é a largura de banda do canal de comunicação. Quanto maior a quantidade de dados a serem transmitidos, maior a largura de banda necessária e maior o aquecimento do chip devido à dissipação de potência.

Esta tese aborda sistemas de processamento de sinais neurais extracelulares que tem como objetivo reduzir a quantidade de dados a serem transmitidos e assim viabilizar a transmissão sem fio. Para poder ser integrados dentro do chip implantável, esses sistemas de processamento devem estar otimizados em termos de área e consumo de potência. Dois processamentos encontrados na pesquisa de interfaces neurais implantáveis são a detecção de impulsos elétricos e a separação de impulsos elétricos (Spike Sorting). Nesta tese apresentam-se soluções para esses tipos de processamentos visando a implementação mediante tecnologia CMOS (Complementary Metal Oxide Semiconductor).

Para o caso da detecção de impulsos elétricos (spikes), nesta tese apresenta-se uma alternativa de implementação em hardware de um operador matemático conhecido como operador não linear de energia (NEO do inglês Nonlinear Energy Operator) ou operador Teager. Através da aplicação desse operador a um sinal neural evidenciase a presença de spikes e atenua-se o ruído. Uma das características inovadoras da implementação apresentada nesta tese é a utilização de um circuito elevador ao quadrado que consiste de apenas três transistores, como bloco funcional básico para a realização da operação NEO. O circuito NEO desenvolvido consome 300 pJ no processamento de um spike e foi caracterizado por simulação até em $30 \mathrm{kHz}$, frequência que é compatível com as taxas de amostragem encontradas na literatura.

O outro processamento abordado nesta tese, conhecido como separação de impulsos elétricos ou Spike Sorting, consiste no agrupamento dos impulsos elétricos re- 
gistrados por um eletrodo em categorias, de maneira que em uma categoria estejam os impulsos gerados por um mesmo neurônio. Em outras palavras, o objetivo é reconhecer quais dos impulsos elétricos medidos pelo eletrodo pertencem a um mesmo neurônio, sendo possível que vários neurônios influenciem na medida realizada por um único eletrodo. Uma solução para a separação de impulsos, apropriada no contexto de sistemas implantáveis, é o template matching. Essa técnica baseia-se na geração de modelos (templates) durante uma fase incial ao final da qual o número de templates gerados corresponde ao número de neurônios identificados pelo eletrodo. Numa fase seguinte, o sistema associa cada impulso elétrico detectado a um dos modelos inicialmente gerados. Nesta tese propõe-se um sistema de classificação que executa essa segunda fase do processo de spike sorting.

Nesta tese apresenta-se o projeto de um sistema de classificação de spikes baseado na técnica template matching, implementado com tecnologia CMOS. A implementação proposta nesta tese baseia-se na representação de amostras analógicas mediante o tempo. Esse tipo de representação de sinais analógicos mediante atrasos de pulsos digitais está sendo muito utilizado como alternativa à representação no domínio da tensão, da corrente ou da carga elétrica. A vantagem desse tipo de representação é que não se vê severamente afetada pela redução da tensão de alimentação dos circuitos integrados fabricados em tecnologias submicrométricas. A taxa de acerto na classificação do sistema desenvolvido é maior que $99 \%$ inclusive considerando um offset de até $20 \mathrm{mV}$ no comparador de saída.

Os circuitos apresentados neste trabalho foram projetados considerando dispositivos da tecnologia TSMC de 90nm.

Palavras-chave: Interfaces neurais implantáveis, detecção de impulsos elétricos, separação de impulsos elétricos. 


\section{Abstract}

Neural signals recording through implantable microelectrode arrays in cortex extracellular medium has become an experimental paradigm for neuroscience. Moreover, recent research about motor neuroprostheses has shown that it is possible to decode motor commands from the signals recorded in the cerebral cortex extracellular medium. In both situations, experimental neuroscience and motor neuroprostheses development, one of the issues encountered in the state-of-the-art is the use of integrated circuits (chips) implanted in the brain. In these chips, neural signals measured with microelectrodes are amplified, filtered, processed, and transmitted to an external computer through wires that run through the skull. There is interest in developing implantable chips that transmit signals to the external computer without the need for wires passing through the skull. In the survey of the state-of-the-art it has found the use of such implantable wireless chips in rats and monkeys, but until the date of this writing we have not found reports of application in humans. One of the aspects that must be taken into account in the development of wireless implantable neural interfaces is the bandwidth of the communication channel. The greater the amount of data to be transmitted, the greater the bandwidth required and higher chip heating due to power dissipation.

This thesis deals with extracellular neural signals processing systems that aim to reduce the amount of data to be transmitted and in this way to enable wireless transmission. In order to integrate them into an implantable chip, those processing systems must be optimized in terms of area and power consumption. Two processes found in the research of implantable neural interfaces are spike detection and spike sorting. In this thesis solutions for these types of processing are presented considering their implementation by CMOS (Complementary Metal Oxide Semiconductor).

For the case of spike detection in this thesis it is presented an alternative for the hardware implementation of a mathematical operator known as NEO (Nonlinear Energy Operator). Through the application of this operator to a neural signal the presence of spikes becomes evident and the noise is attenuated. One of the innovative characteristics of the implementation presented in this thesis is the use of a squarer circuit which consists of only three transistors, as a basic function block for performing operation of NEO. NEO circuit consumes $300 \mathrm{pJ}$ in processing a spike, and was characterized by simulation up to $30 \mathrm{kHz}$, frequency which is compatible with sampling rates found in the literature.

The other processing discussed in this thesis, known as Spike Sorting, is the grouping of electrical impulses recorded by an electrode into categories so that the spikes belonging to the same category were generated by a single neuron. In other words, the goal is to recognize which of the spikes measured by the electrode belong to the same neuron, given that it is possible that several neurons influence the measure performed by a single electrode. A solution for the Spike Sorting suitable in the context of 
implantable systems, is the template matching. This technique is based on generating templates during an initial phase at the end of which the number of generated templates corresponds to the number of neurons identified by the electrode. In the next phase, the system associates each detected spike to one of the templates generated initially. In this thesis it is proposed a classification systems which performs that second phase of the spike sorting process.

This thesis presents the design of a spike classification system based on template matching technique, implemented in CMOS technology. The processing proposed in this work is based on the time-based representation of the analog samples. This kind of representation of analog signals by delays of digital pulses is being widely used as an alternative to the classical representation of samples by voltage, current or electric charge. The advantage of this time-mode representation is that it is not severely affected by reduced supply voltage of integrated circuits manufactured in sub-micrometer technologies. The classification hit rate of the developed system is greater than $99 \%$ even when an offset of $20 \mathrm{mV}$ is assumed for the output comparator.

All the circuits presented in this work were designed using devices from TSMC $90 \mathrm{~nm}$ technology.

Keywords: Implantable neural interfaces, spike detection, spike sorting. 


\section{Lista de Figuras}

1.1 Principais fatos no desenvolvimento de neuropróteses motoras. Adaptado de (JACKSON, 2012) . . . . . . . . . . . . . . . . . 19

1.2 Matt Nagle, paciente tetraplégico, controlando o ponteiro do mouse através de uma interface implantável de registro neural. Experimento relatado em (HOCHBERG et al., 2006). Figura extraída de (ABBOTT, 2006)

1.3 Paciente tetraplégica bebendo de uma garrafa de café mediante um braço robô controlado pelos sinais neurais registrados no córtex motor. Imagem extraída de (HOCHBERG et al., 2012) . . . . . . . . . . . . . 21

1.4 Paciente tetraplégica comendo uma barra de chocolate mediante um braço robô controlado pelos sinais neurais registrados no córtex motor. Experimento relatado em (COLLINGER et al., 2013). Imagem extraída do site http://www.slashgear.com . . . . . . . . . . . .

1.5 Participante do experimento realizado pela equipe do professor Richard Andersen. Imagem extraída do site https://www.vis.caltech.edu/media .

1.6 Imagem extraída do site do pesquisador Benjamin I. Rapoport (benjaminirapoport.com) . . . . . . . . . . . . . . .

2.1 Representação simplificada de um canal de leitura, processamento e transmissão de sinais neurais extracelulares . . . . . . . . . . 38

2.2 Diagrama de blocos de uma interface neural implantável multicanal . . 38

2.3 Diagrama de blocos do sistema apresentado em (AKIN; NAJAFI; BRADLEY, 1998). Figura extraída do artigo (AKIN; NAJAFI; BRADLEY, 1998). . . . . . 40

2.4 Sonda ativa utilizada no sistema de Olsson e Wise. Imagem extraída de (OLSSON-III; WISE, 2005) . . . . . . . . . . . . . . . . . . . . 40

2.5 Diagrama de blocos do sistema apresentado em (OLSSON-III; WISE, 2005). Imagem extraída de (OLSSON-III; WISE, 2005) . . . . . . . . . . 41 
2.6 Diagrama de blocos internos e periféricos externos do sistema apresentado em junho 2007 pelo Center for Wireless Integrated MicroSystems (WIMS) da universidade de Michigan. Imagem extraída de (SODAGAR et al., 2007). NPU (Neural Processor Unit), BPF (Band Pass Filter) e POR(Power On Reset). . . . . . . . . . . . . . . . . . . . . . . .

2.7 Diagrama de blocos do sistema desenvolvido pela equipe do professor Wentai Liu da Universidade de Califórnia Santa Cruz. Imagem extraída do artigo (CHAE et al., 2009) . . . . . . . . . . . . . . . . . .

2.8 Ilustração do sistema apresentado em (ZOU et al., 2013). Imagem extraída do artigo (ZOU et al., 2013). . . . . . . . . . . . . . . . . . .

2.9 Diagrama de blocos do sistema apresentado em (ZOU et al., 2013). Imagem extraída do artigo (ZOU et al., 2013). . . . . . . . . . . . . . . .

2.10 Digrama de blocos e ilustração do sistema apresentado em (MULLER et al., 2014). Imagem extraída do artigo (MULLER et al., 2014). . . . . . . . .

2.11 llustração de um sistema multi-eletrodo de medida de sinais neurais extracelulares. Imagem extraída de (NG; XU, 2015). . . . . . . . . . . . .

3.1 Ilustração do método de detecção de spike por aplicação de limiar simples 52

3.2 Modos de detecção suportados pelo processador apresentado em (soDAGAR; WISE; NAJAFI, 2006). (a) Positivo (b) Negativo (c) Bifásico. Imagem extraída de (SODAGAR; WISE; NAJAFI, 2006). . . . . . . . . . . .

3.3 Estratégia para geração de limiar de comparação apresentada em (HARRISON, 2003). Imagem extraída de (HARRISON, 2003). . . . . . . . . . 53

3.4 Implementação analógica de detecção de spikes com estágio de préênfase. Imagem extraída de (HISENI et al., 2009). . . . . . . . . . . . .

3.5 Ilustração da ideia que a amplitude não é parâmetro suficiente para caracterizar um spike . . . . . . . . . . . . . . . . . .

3.6 Implementação analógica do NEO apresentada em (HOLLEMAN et al., 2008). Imagem extraída de (HOLLEMAN et al., 2008).

3.7 Implementação analógica do NEO apresentada em (LI; XU, 2011). Imagem extraída de $(\mathrm{LI} ; \mathrm{XU}, 2011) \ldots \ldots \ldots \ldots \ldots$ 
3.8 Implementação analógica do NEO. Imagem extraída do artigo (GOSSELIN; SAWAN, 2009).

3.9 Ilustração do processo de Spike Sorting. A partir do sinal registrado por um único eletrodo (figura superior) os impulsos elétricos são detectados mediante um processo de limiarização e, finalmente, separados. Figura adaptada de (QUIROGA; NADASDY; BEN-SHAUL, 2004) . . . . . . . . . .

3.10 llustração do processo deSpike Sorting criada por Karkare e Gibson (KARKARE; GIBSON; MARKOVIC, 2013). Imagem extraída de (KARKARE; GIBSON; MARKOVIC, 2013). . . . . . . . . . . . . . . . . .

3.11 Proposta para utilização dos sistemas desenvolvidos nesta tese dentro de um sistema de processamento de sinais neurais . . . . . . . . . . .

4.1 Diagrama de blocos do sistema de detecção baseado no operador NEO. O bloco denominado VTC é um conversor de tensão a corrente (Voltage to current converter) . . . . . . . . . . . . . . . . . . .

4.2 Resultado da aplicação do NEO implementado em Matlab aos sinais e aos valores absolutos dos sinais do banco de dados Waveclus. Sinais com ruídos de $5 \%, 10 \%, 15 \%$ e $20 \% \ldots \ldots \ldots$. . . . . . . .

4.3 Resultado da aplicação do NEO implementado em Matlab aos sinais e aos valores absolutos dos sinais do banco de dados Waveclus. Sinais com ruídos de $25 \%, 30 \%, 35 \%$ e $40 \%$. . . . . . . . . . . . . . . .

4.4 Circuito elevador ao quadrado. Considera-se entrada à corrente drenada do transistor M2 $\left(I_{i n}\right)$ e como saída, à corrente fornecida pelo transistor M3 $\left(I_{o u t}\right) \ldots \ldots \ldots \ldots \ldots \ldots$

4.5 Circuito empregado para simular o elevador ao quadrado e determinar um valor apropriado de largura de canal (W) . . . . . . . . . . .

4.6 Coeficiente de correlação linear entre a corrente de saída, obtida mediante a simulação do circuito da figura 4.5, e o quadrado da corrente de entrada em função do comprimento de canal dos transistores PMOS. O comprimento de canal foi fixado em $1 \mu \mathrm{m} . \ldots \ldots \ldots \ldots \ldots$

4.7 Esquema elétrico do circuito Operador NEO formado por elevadores ao quadrado do tipo mostrado na figura $4.4 . \ldots \ldots$. . . . . . . . 74

4.8 Princípio de operação do conversor de tensão a corrente $\ldots \ldots$. . . 76 
4.9 Estratégia para gerar valor absoluto da corrente Iout . . . . . . . . . . . 77

4.10 (a) Sinal sintético aplicado ao circuito NEO. O desvio padrão do ruído é igual ao $25 \%$ da amplitude máxima do sinal. (b) Resposta do circuito NEO à aplicação do sinal sintético escolhido. . . . . . . . . . . . . . . . . . . 78

4.11 Curva ROC para o sinal original (linha em azul) e para o sinal de saída do circuito NEO (linha em vermelho).

4.12 Resposta do circuito a um sinal real. O sinal de saída foi escalado de maneira que o pico máximo coincida com o pico máximo do sinal de entrada.

5.1 Diagrama de blocos simplificado do sistema de classificação de spikes desenvolvido. VTC é o conversor de tensão a tempo (Voltage-to-Time Converter), WTA é o circuito que determina qual das três entradas é menor (Winner Take All) . . . . . . . . . . . . . . . . . . . . . . . .

5.2 Representação de amostras baseada em tempo. Os atrasos dos pulsos em relação a $t_{r s t}$ são proporcionais às tensões de entrada dos VTC. . .

5.3 Representação de amostras baseada em tempo mediante um único VTC. 85

5.4 Conversor de tensão a tempo baseado em modulação PWM (Pulse Width Modulator. . . . . . . . . . . . . . . . . . . . . . . . . . . . . . . . 86

5.5 Conversor de tensão a tempo apresentado em (MOHAMAD et al., 2013). Neste caso uma das tensões de entrada do comparador é constante o qual facilita o seu projeto. . . . . . . . . . . . . . . . . . .

5.6 Circuito subtrator baseado em tempo. VTC: Voltage to Time Converter, DE: Delay Element. . . . . . . . . . . . . . . . . . . . . . . . . . . . . 89

5.7 Diagrama de tempos da operação do circuito subtrator . . . . . . . . . . 89

5.8 Circuito subtrator baseado em tempo com saída na forma de tensão. . 91

5.9 Programação do circuito subtrator para calcular diferenças em relação a $V_{y} \ldots \ldots \ldots \ldots$. . . . . . . . . . . . . . . . . . . . . . . . . . . . 91

5.10 Diagramas de tempos obtidos mediante a simulação do loop de programação do circuito subtrator para calcular diferenças em relação a

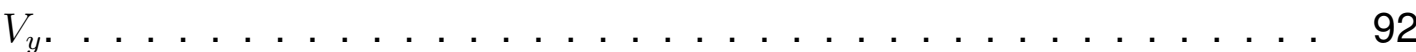


5.11 Esquema elétrico do elemento de atraso (DE da figura 5.9) utilizado neste trabalho.

5.12 Circuito que calcula a distância de Manhattan baseado no uso de uma fonte de corrente para cada canal. As correntes são integradas no capacitor de saída gerando uma tensão proporcional à distância de Manhattan. 95

5.13 Circuito que calcula a distância de Manhattan com uso compartilhado de VTC e de integrador de saída . . . . . . . . . . . . . . . . 96

5.14 Spikes de três neurônios diferentes, presentes no sinal utilizado na simulação do sistema de classificação. . . . . . . . . . . . . . . . . 97

5.15 Resultados de simulação da aplicação de um spike da classe 1 ao sistema.

5.16 Resultados de simulação da aplicação de um spike da classe 2 ao sistema.

5.17 Resultados de simulação da aplicação de um spike da classe 3 ao sistema.

5.18 Precisão da classificação em função da mínima tensão que o comparador de saída pode reconhecer . . . . . . . . . . . . . . . . . . 100

C.1 Esquema do circuito conversor de tensão a corrente. . . . . . . . . . . . 115

C.2 Esquema do circuito NEO . . . . . . . . . . . . . . . . . . . . 116

D.1 Layout do circuito conversor de tensão a corrente. . . . . . . . . . 117

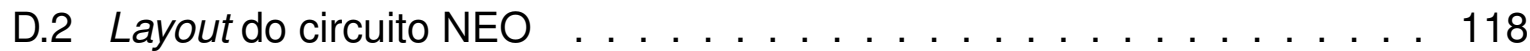




\section{Lista de Tabelas}

1.1 Lista de publicações recentes que abordam os problemas de detecção e separação de impulsos elétricos neurais em interfaces neurais implantáveis . . . . . . . . . . . . . . . . . . . . . . . . 29

1.2 Lista de bancos de dados de sinais neurais encontrados no estudo do estado da arte . . . . . . . . . . . . . . . . . 33

2.1 Conversores analógico-digital utilizados em sistemas implantáveis wireless de registro neural . . . . . . . . . . . . . . . . . . . . 49

4.1 Parâmetros de detecção: tpr (Itrue positive rate) e fpr (false positive rate) 68

4.2 Taxas de detecção para diferentes níveis de ruído. O limiar de comparação foi fixado em 8nA. . . . . . . . . . . . . . . . . . 80

4.3 Comparação com outros trabalhos . . . . . . . . . . . . . . . 81 


\section{Sumário}

1 Introdução e o estado da arte 16

1.1 Interfaces neurais implantáveis $\ldots \ldots \ldots \ldots \ldots \ldots$

1.1.1 Interfaces neurais implantáveis que realizam a leitura de sinais

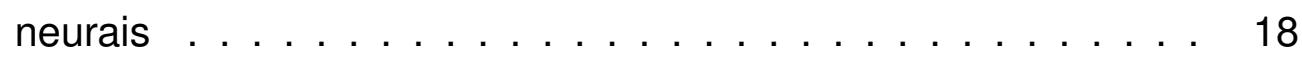

1.1.2 Interfaces que estimulam o sistema nervoso $\ldots \ldots \ldots .25$

1.1.3 Interfaces neurais bidirecionais . . . . . . . . . . . 26

1.1.4 Rumo da pesquisa em interfaces neurais implantáveis . . . . . 26

1.2 Objetivos do projeto de doutorado $\ldots \ldots \ldots \ldots \ldots \ldots$

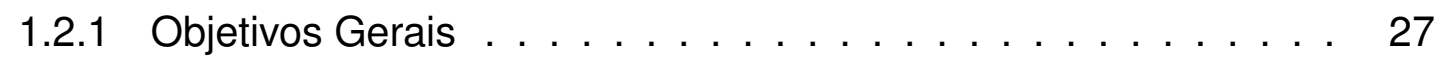

1.2 .2 Objetivos Específicos . . . . . . . . . . . . . . . . 29

1.3 Estratégia de avaliação dos sistemas desenvolvidos neste trabalho . . 31

1.3.1 Banco de dados escolhido para a avaliação dos sistemas desenvolvidos nesta tese . . . . . . . . . . . . . . . . 33

1.4 Estrutura da tese . . . . . . . . . . . . . . . . . 34

2 Sistemas implantáveis de leitura, processamento e transmissão sem fio de sinais neurais extracelulares: descrição e o estado da arte 37

2.1 Sistemas implantáveis de registro neural sem fio - o estado da arte . . . 39

2.2 Amplificação de sinais neurais extracelulares . . . . . . . . . . 47

2.3 Conversores analógico-digital de sinais neurais extracelulares _. . . 49

2.4 Processamento de sinais neurais extracelulares em circuitos integrados implantáveis . . . . . . . . . . . . . . . . 49 
3 Detecção e separação de impulsos elétricos neurais em circuitos integrados implantáveis - estado da arte

3.1 Detecção de impulsos elétricos neurais $\ldots \ldots \ldots \ldots \ldots$. . . . . 51

3.1.1 Aplicação de pré-ênfase ao sinal . . . . . . . . . . . . . . . 54

3.1.2 Operador não linear de energia . . . . . . . . . . . . . . . . 55

3.2 Separação de impulsos elétricos neurais $\ldots \ldots \ldots$. . . . . . . . 59

3.3 Proposta para utilização dos sistemas de detecção e classificação de spikes . . . . . . . . . . . . . . . . . . . . . . . 62

4 Desenvolvimento de um sistema de detecção de impulsos elétricos neurais para uma interface neural implantável

4.1 Análise da aplicação do operador Teager ao valor absoluto do sinal neural extracelular . . . . . . . . . . . . . . . . . . 65

4.2 Estratégia para realizar o operador Teager baseada no uso de circuitos elevadores ao quadrado . . . . . . . . . . . . . . . . . . . 69

4.3 Circuito elevador ao quadrado baseado em transistores MOS que operam na região sublimiar . . . . . . . . . . . . . . . . . . 70

4.4 Circuito que realiza o operador Teager em modo corrente . . . . . . . 74

4.5 Circuito que gera uma corrente proporcional ao valor absoluto do sinal de tensão de entrada . . . . . . . . . . . . . . . . . . 76

4.6 Simulações elétricas do sistema de detecção desenvolvido $\ldots$. . . . . 78

5 Sistema de classificação de impulsos elétricos neurais 82

5.1 Representação de sinais como atrasos entre pulsos digitais . . . . . . . 84

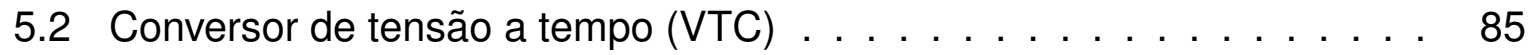

5.3 Cômputo da distância de Manhattan . . . . . . . . . . . . . . 88

5.3.1 Circuito subtrator baseado em tempo $\ldots \ldots \ldots$. . . . . 88

5.3.2 Programação do circuito subtrator . . . . . . . . . . . . . 91

5.4 Circuito que calcula a distância de Manhattan . . . . . . . . . . . . 95 
5.5 Simulação do sistema de classificação de impulsos elétricos desenvolvido nesta tese . . . . . . . . . . . . . . . . . . . . . . . . . 97

6 Conclusões e perspectivas 102

6.1 Sobre o estado da arte e o rumo da pesquisa em interfaces neurais implantáveis . . . . . . . . . . . . . . . . . . . . . . . . 102

6.2 Sobre o sistema de detecção de impulsos elétricos neurais desenvolvido nesta tese . . . . . . . . . . . . . . . . . . . . 103

6.3 Sobre o sistema de classificação de impulsos elétricos neurais desenvolvido nesta tese . . . . . . . . . . . . . . . . . . . . . 104

6.4 Propostas para trabalhos futuros . . . . . . . . . . . . . 106

$\begin{array}{ll}\text { Apêndice A - Programas em Matlab } & 107\end{array}$

A.1 Extração e filtrado de sinais do banco de dados Waveclus . . . . . . . 107

A.2 Extração de instantes de ocorrência de spikes em um sinal do banco de dados Waveclus . . . . . . . . . . . . . . . . . . 107

A.3 Geração de tabela de resultados do NEO implementado em matlab e aplicado a sinais do banco de dados Waveclus . . . . . . . . . . 107

A.4 Extração e filtrado de sinais do banco de dados Osort . . . . . . . . . . 108

A.5 Extração de instantes de ocorrência de spikes em um sinal do banco de dados Osort . . . . . . . . . . . . . . . . . . . . . . . . . 108

A.6 Construção de curva ROC . . . . . . . . . . . . . . . . . . . . 108

Apêndice B - Programas em OCEAN $\quad 110$

B.1 Programa para a explorar o espaço de projeto do circuito elevador ao quadrado . . . . . . . . . . . . . . . . . . . . . 110

B.2 Programa para o a simulação do circuito NEO em regimen DC . . . . 111

B.3 Programa para a obter a tensão de calibração dos elementos de atraso 111

B.4 Programa para obter os parâmetros de deteção com base nos resultados da simulação do detector de spikes . . . . . . . . . . . . . . . 112 


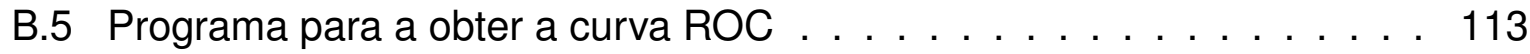

B.6 Programa para simulação do cômputo da distância entre dos vetores de grandezas analógicas . . . . . . . . . . . . . . . . . . . . 114

Apêndice C - Esquemas elétricos em Cadence $\quad 115$

C.1 Esquema do circuito conversor de tensão a corrente . . . . . . . . . 115

C.2 Esquema do circuito NEO . . . . . . . . . . . . . . . . . . . 116

$\begin{array}{ll}\text { Apêndice D - Layouts dos circuitos } & 117\end{array}$

D.1 Layout do circuito conversor de tensão a corrente . . . . . . . . . . 117

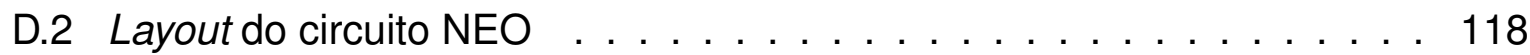

$\begin{array}{ll}\text { Apêndice E - Nestlists dos circuitos projetados } & 119\end{array}$

E.1 Netlist do circuito NEO . . . . . . . . . . . . . . . . . . . . . 119

E.2 Netlist do circuito bloco com 4 elementos de atrasso . . . . . . . . 122

E.3 Netlist da simulação do calculo de distância . . . . . . . . . . . . . . 123 


\section{Introdução e o estado da arte}

O presente capítulo foi elaborado com a finalidade de delimitar os alcances do trabalho de doutorado e salientar sua relevância científica.

Esta tese de doutorado aborda temas relacionados aos circuitos integrados implantáveis - denominados interfaces neurais implantáveis, segundo tradução do termo encontrado nos artigos em inglês: Implantable Neural Interfaces - que interagem com o sistema nervoso. Neste capítulo, apresenta-se uma revisão do estado da arte das interfaces neurais implantáveis, focando-se principalmente em aplicações desses sistemas na recuperação parcial ou total de funções sensoriais ou motoras em pacientes que apresentaram limitações relacionadas ao sistema nervoso ou que sofreram algum acidente que levou à perda de tais funções.

Após essa revisão do estado da arte, enunciam-se os objetivos do projeto de doutorado e demonstra-se sua relevância científica. Para tal fim, apresentam-se exemplos encontrados na revisão da literatura científica que evidencia o esforço realizado por vários centros de pesquisa no desenvolvimento de soluções para os problemas abordados nesta tese.

Posteriormente, discutem-se os procedimentos realizados para avaliar os sistemas desenvolvidos neste trabalho de doutorado, valendo-se de exemplos obtidos em trabalhos similares. Apresentam-se também as bases de dados de sinais neurais utilizadas para simular os sistemas desenvolvidos neste doutorado. A estrutura desses conjuntos de sinais bem como os procedimentos realizados para a sua geração serão objetos de análise.

Finaliza-se este capítulo com uma descrição da estrutura e organização da tese, deixando bem evidente quais partes correspondem à revisão teórica e do estado da arte e quais correspondem ao trabalho desenvolvido pelo autor como parte do projeto de doutorado. 


\subsection{Interfaces neurais implantáveis}

As interfaces neurais são sistemas que operam na intersecção entre o sistema nervoso e um dispositivo artificial interno ou externo. Quando utilizadas para prover uma extensão artificial que recupera ou aumenta alguma função do sistema nervoso, são chamadas de próteses neurais. No caso em que são projetadas para serem implantadas no corpo humano, emprega-se a denominação interfaces neurais implantáveis. $O$ estudo e o desenvolvimento desse tipo de sistemas pertencem à disciplina Engenharia Neural ou Neuroengenharia.

A Engenharia Neural é uma área multidisciplinar que trata do uso de ferramentas da engenharia para interagir com o sistema nervoso, desde o registro e processamento de sinais neurais até a restauração e melhora de funções. Esta área de pesquisa tem atraído grande interesse por parte da comunidade científica, fato comprovado pela existência de importantes revistas especializadas, tais como Journal of Neural Engineering, Journal of Neuroengineering and Rehabilitation e Journal of Neuroscience Methods.

Embora exista uma grande variedade de exemplos de aplicação das interfaces neurais implantáveis, reconhecem-se como objetivos principais dessa tecnologia a restauração de funções em pessoas que possuem limitações físicas e o entendimento do funcionamento do sistema nervoso humano (HE, 2013).

As interfaces neurais implantáveis são ferramentas de uma tecnologia mais abrangente conhecida como Brain-Computer Interfaces (BCI) (GRAIMANN; ALLISON; PFURTSCHELLER, 2010). As primeiras demonstrações da tecnologia $\mathrm{BCl}$ ocorreram na década de 1960. Na primeira, Grey Walter utilizou o eletroencefalograma (EEG) registrado no escalpo de uma pessoa para controlar um projetor de slides em 1964 (GRAIMANN; ALLISON; PFURTSCHELLER, 2010). Na segunda, Eberhard Fetz ensinou macacos - que receberam comida como recompensa - a controlar uma agulha de medida, mudando a taxa de disparos de um neurônio do córtex (FETZ, 1969).

Distinguem-se três tipos de interfaces neurais segundo seu fluxo de informação: as interfaces que realizam a leitura de impulsos elétricos do sistema nervoso, conhecidas como sistemas de registro neural (denominadas Neural Recording Interfaces nos artigos em inglês); as interfaces que aplicam estímulos ao sistema nervoso e, finalmente, as interfaces que registram e estimulam o sistema nervoso, conhecidas como interfaces neurais bidirecionais. Para qualquer dos três tipos, os propósitos das pesquisas 
recentes são a implantação das interfaces e a capacitação de comunicação com dispositivos externos através de canais de comunicação sem fio. A seguir, apresenta-se uma revisão do estado da arte nos três tipos de interfaces.

\subsubsection{Interfaces neurais implantáveis que realizam a leitura de si- nais neurais}

Conhecidas como interfaces de registro neural (nos artigo em inglês são denominadas como Neural Recording Interfaces), estes sistemas têm como objetivo medir sinais neurais através de matrizes de microeletrodos e, após alguns tipos de processamento, enviar os resultados a um computador externo.

O registro crônico de sinais neurais através de matrizes de microeletrodos tem se tornado, por um lado, um importante paradigma experimental nos estudos sobre o funcionamento do cérebro. A leitura de sinais neurais extracelulares de vários canais é utilizada, em experimentos de neurociência, para se estudar redes neurais complexas em animais com liberdade de movimento (RUTISHAUSER; SCHUMAN; MAMELAK, 2006), (SANTHANAM et al., 2007), (SCHWARTZ et al., 2014), (MESTAIS et al., 2015). Por outro lado, experimentos com interfaces cérebro-máquina que utilizam sistemas de registro neural têm provado que é possível antecipar os movimentos dos braços analisando-se registros simultâneos de vários neurônios. Portanto, os sistemas de registro neural são importantes tanto para pesquisas na área de neurociência quanto para o desenvolvimento de neuropróteses motoras.

As interfaces neurais implantáveis que realizam leitura de sinais são utilizadas em duas grandes áreas de pesquisa: o estudo de redes neurais biológicas em animais e o desenvolvimento de neuropróteses motoras.

Geralmente, interfaces que permitem registros de atividade neural possibilitam o controle de algum dispositivo externo por meio de comandos cerebrais. Este é exatamente o caso do controle de braços artificiais que vem obtendo resultados impressionantes. Na figura 1.1, adaptada do artigo (JACKSON, 2012), apresentam-se alguns dos principais fatos que promoveram o desenvolvimento de neuropróteses motoras.

A seguir, apontam-se alguns exemplos notáveis de experimentos envolvendo o uso de neuropróteses motoras em humanos. Nesses experimentos, utilizam-se matrizes de microeletrodos implantados no córtex. Após a amplificação, filtragem e conversão ao domínio digital, os sinais são transmitidos através de cabos que atravessam o crânio até um computador externo. 


\section{6}

Registro de neurônios

do córtex motor

em macacos acordados
1982

Descobre-se que neurônios do córtex motor de macacos são sintonizados para a direção de movimentos
1970

Decodificação de parâmetros

de movimento a partir da atividade de conjuntos de neurônios corticais
2000

Controle Motor Malha Aberta

em macacos
2006
Braingate

Demonstração

em humanos
1966 1970 1974

1978 1982 1986

$\prod_{1994}^{1090}$

Registros neurais com tecnologia de eletrodos em silício

2012

Controle de braço robô para

alcançar e agarrar objetos por pacientes com paralisia

Pesquisa em mecanismos neurais que controlam os movimentos dos braços

Pesquisa em desenvolvimento de eletrodos

Interfaces neurais em macacos

Interfaces neurais em humanos

Figura 1.1: Principais fatos no desenvolvimento de neuropróteses motoras. Adaptado de (JACKSON, 2012) 


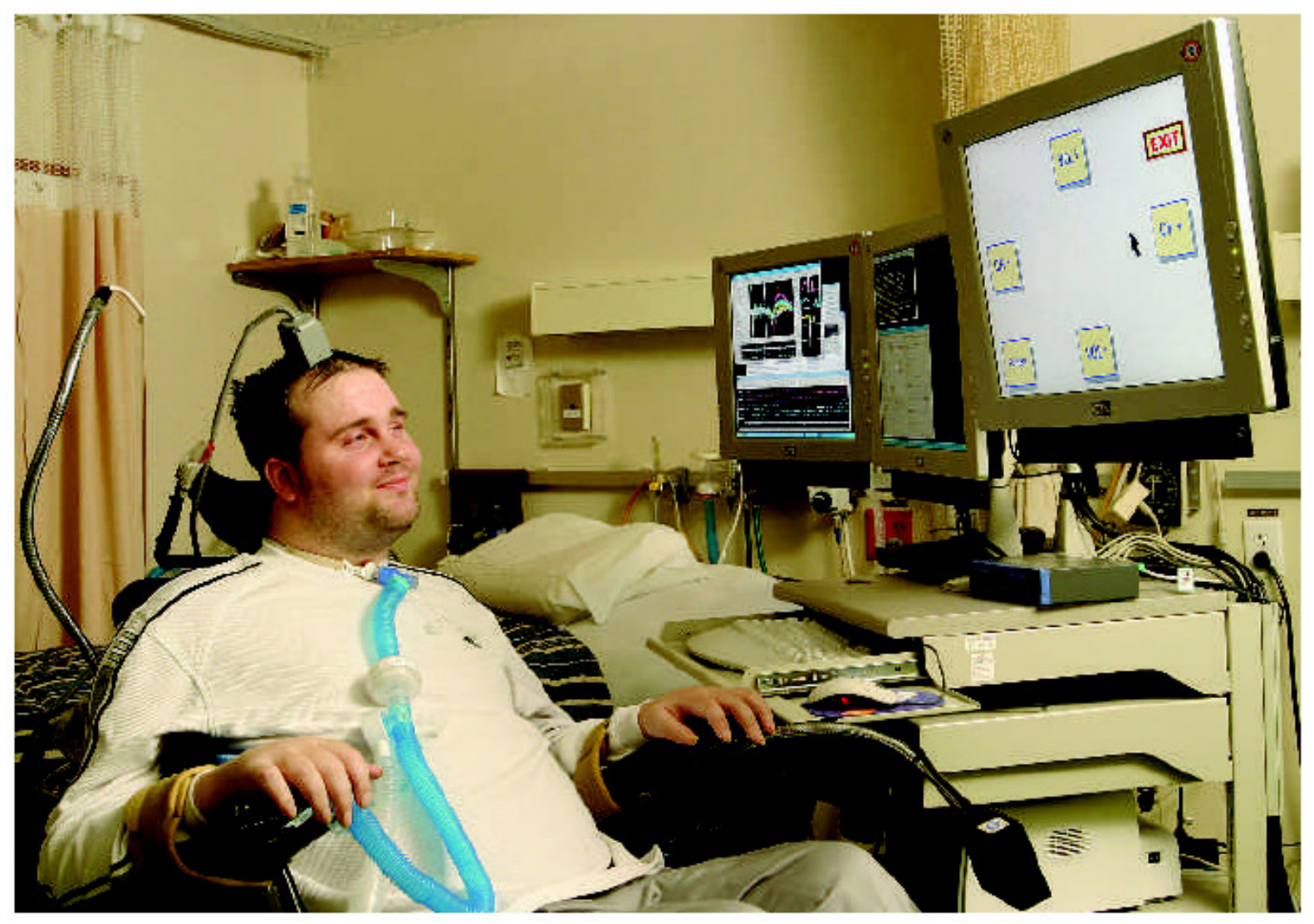

Figura 1.2: Matt Nagle, paciente tetraplégico, controlando o ponteiro do mouse através de uma interface implantável de registro neural. Experimento relatado em (HOCHBERG et al., 2006). Figura extraída de (ABBOTT, 2006)

Em 2006, uma equipe constituída por pesquisadores da Universidade de Brown e da empresa Cyberkinetics Neurotechnology Systems, liderada pelo professor John Donogue, implantou 96 eletrodos no córtex motor de Matt Nagle, um paciente tetraplégico. Demonstrou-se, através de tal experimento, que o paciente fora capaz de controlar um ponteiro de mouse através do pensamento (HOCHBERG et al., 2006).

A matriz de 96 eletrodos foi implantada no córtex motor primário. O paciente tinha sofrido uma lesão na medula espinhal dorsal três anos antes do experimento. Concluiu-se que as intenções de movimento geram padrões de atividade neural que podem ser decodificados para executá-las. 


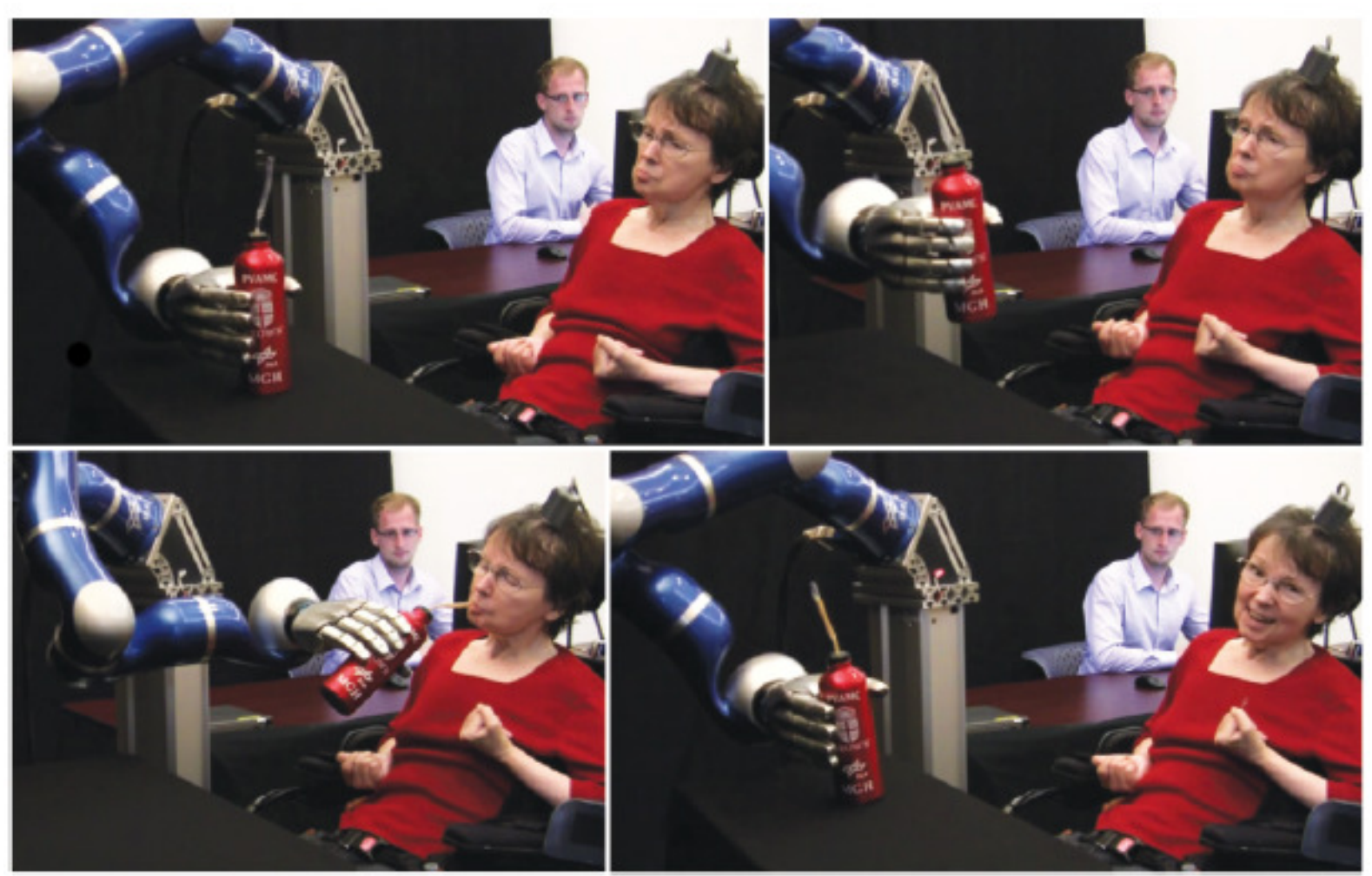

Figura 1.3: Paciente tetraplégica bebendo de uma garrafa de café mediante um braço robô controlado pelos sinais neurais registrados no córtex motor. Imagem extraída de (HOCHBERG et al., 2012)

A equipe do professor Donogue apresentou em 2012 resultados de outro experimento envolvendo seres humanos (HOCHBERG et al., 2012). As pacientes foram mulheres de 58 anos e 66 anos identificadas no artigo como S3 e T2, respectivamente. Ambas tinham sofrido acidente vascular cerebral que, como consequência, resultou em tetraplegia e anartria. O projeto foi denominado BrainGate2 e representou a continuação do BrainGate1, comentado na página anterior.

Os sinais foram coletados mediante uma matriz de microeletrodos de $4 \mathrm{~mm} \times 4 \mathrm{~mm}$ contendo 96 canais. Essa matriz foi implantada na área do córtex motor, identificada como Ml, que corresponde aos comandos de movimento das mãos. Os sinais foram transmitidos através de fios que atravessavam o crânio até um computador externo (ver figura 1.3).

As pacientes puderam, inicialmente, realizar controle do ponteiro de um mouse. Após 6 anos de uso do implante, elas conseguiram controlar um braço robô. 


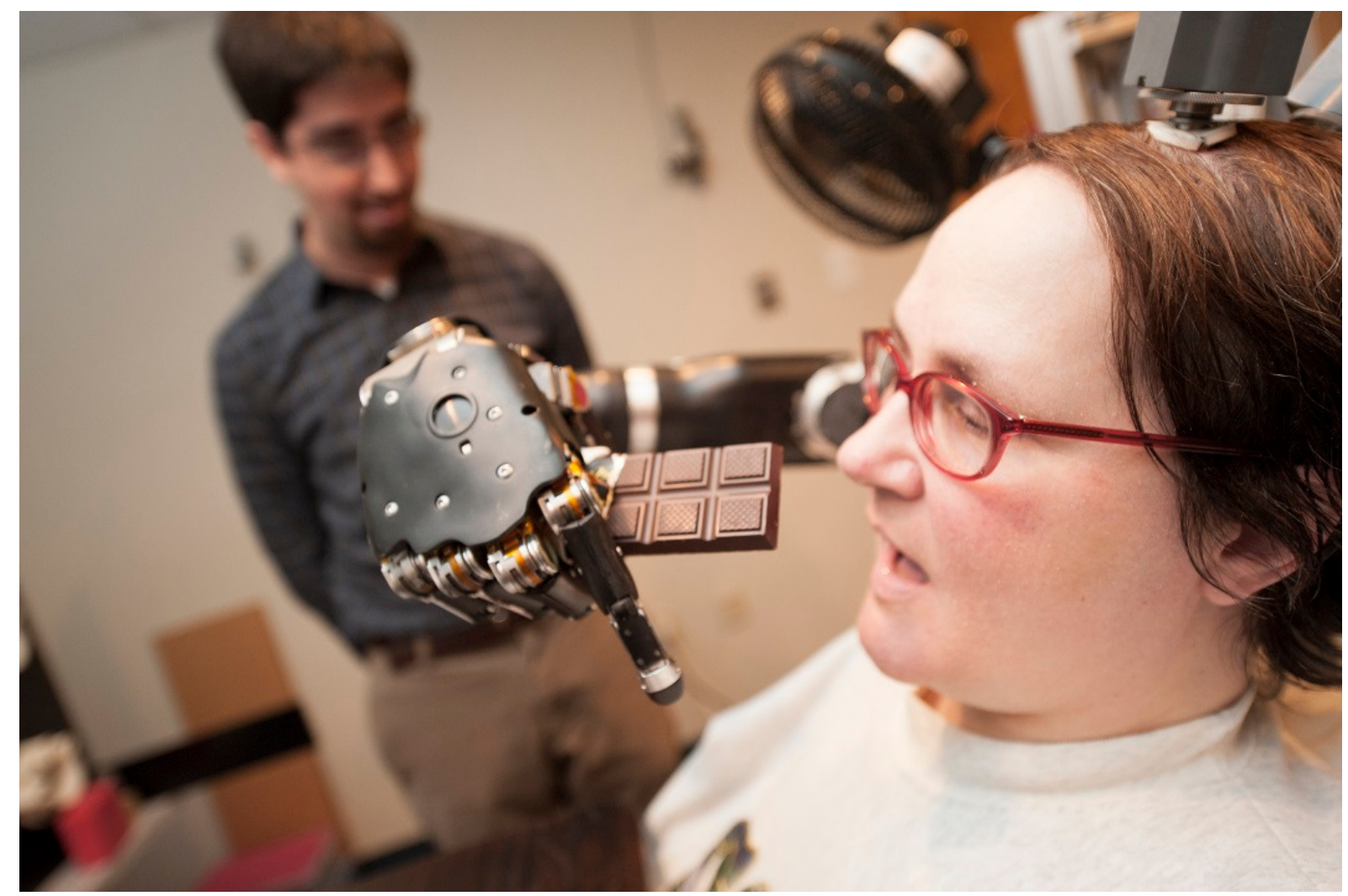

Figura 1.4: Paciente tetraplégica comendo uma barra de chocolate mediante um braço robô controlado pelos sinais neurais registrados no córtex motor. Experimento relatado em (COLLINGER et al., 2013). Imagem extraída do site http://www.slashgear.com

Em 2013, foi publicado um artigo que relatava um experimento onde uma mulher tetraplégica conseguira comer uma barra de chocolate com o auxílio de um braço robô controlado pelos sinais neurais coletados no córtex motor (COLLINGER et al., 2013) (ver figura 1.4).

Apesar do surpreendente resultado obtido, os cientistas não se conformaram e foram em busca de um objetivo mais ambicioso: o desenvolvimento de interfaces cérebro-máquina que operassem em malha fechada. $O$ elemento que fecharia a maIha seria o equivalente artificial do sentido de propriocepção, isto é, a capacidade de determinar a posição relativa dos membros superiores e inferiores sem a necessidade de observá-los. Conceitualmente, a propriocepção artificial pode ser obtida através de sensores que coletam informações sobre pressão, velocidade, aceleração, temperatura, entre outras. Essas informações poderiam ser transmitidas ao sistema nervoso e, portanto, o controle da prótese artificial seria mais preciso. Na prática, porém, a retroalimentação do estado da prótese é muito difícil de ser obtida, como foi discutido em (KWOK, 2013). 


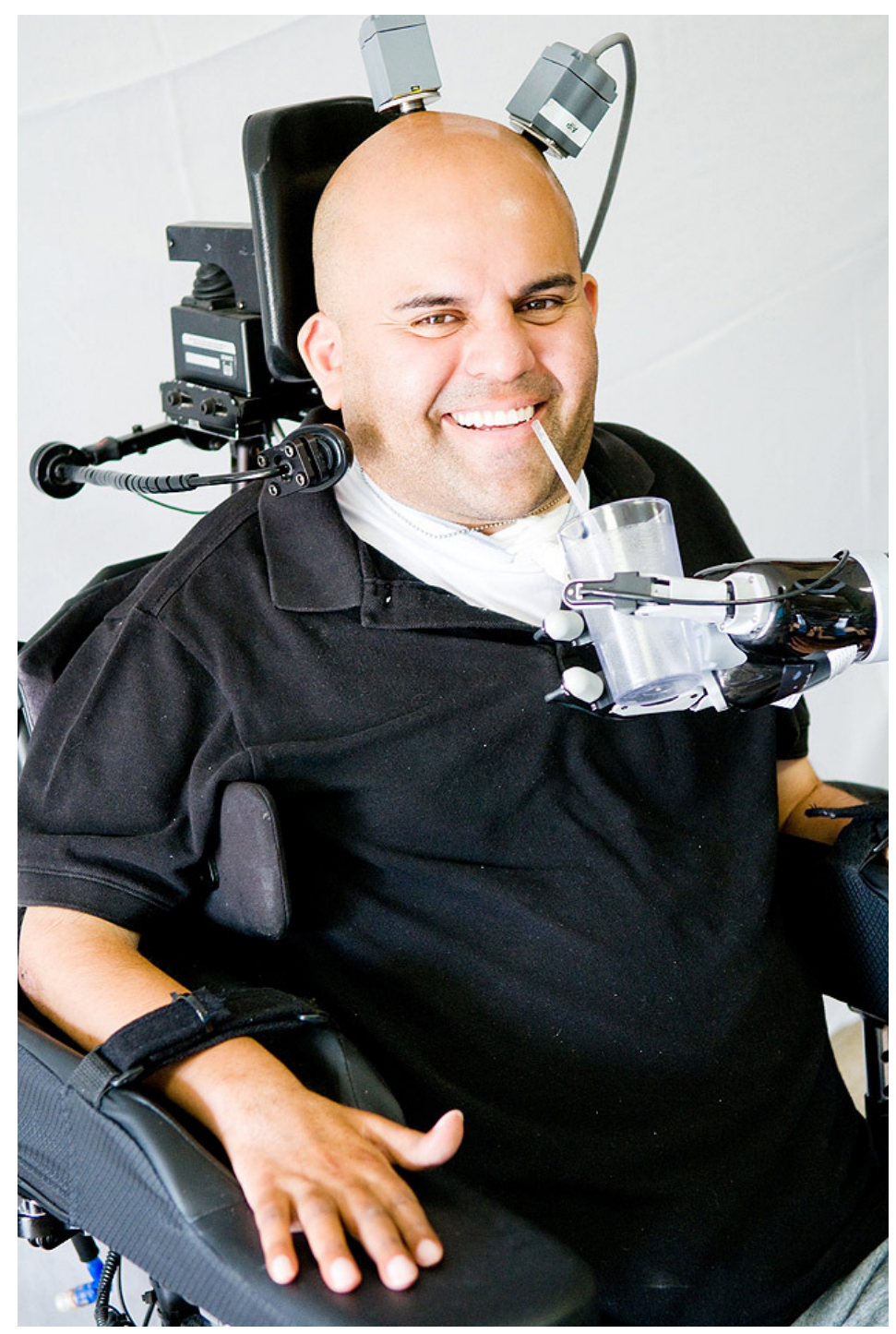

Figura 1.5: Participante do experimento realizado pela equipe do professor Richard Andersen. Imagem extraída do site https://www.vis.caltech.edu/media

Um estudo recente realizado por uma equipe liderada pelo professor Richard Andersen do Instituto de Tecnologia da Califórnia (Calltech) revelou progresso, em relação a experiências anteriores, no controle de um braço robô por um indivíduo tetraplégico (AFLALO et al., 2015). A novidade desse experimento é que ao invés de analisar sinais do córtex motor primário - como é feito na maioria dos casos - os eletrodos foram implantados no córtex parietal posterior. Esta área do cérebro, conhecida como PPC (Posterior Parietal Cortex), é geralmente relacionada ao planejamento do movimento, enquanto o córtex motor primário está associado à execução do movimento. 
Em todos os experimentos que acabamos de apresentar, os sinais registrados no córtex foram transmitidos através de fios que atravessam o crânio até um computador externo. Isso cria, certamente, uma limitação de movimentos ao paciente e promove a aparição de infecções. Atualmente, existe interesse no desenvolvimento de interfaces que não precisem de fios para a transmissão de sinais. Com esse tipo de abordagem, um chip ficaria implantado e ligado à matriz de microeletrodos que estaria em contato com o córtex cerebral. Esse conceito está ilustrado na figura 1.6.

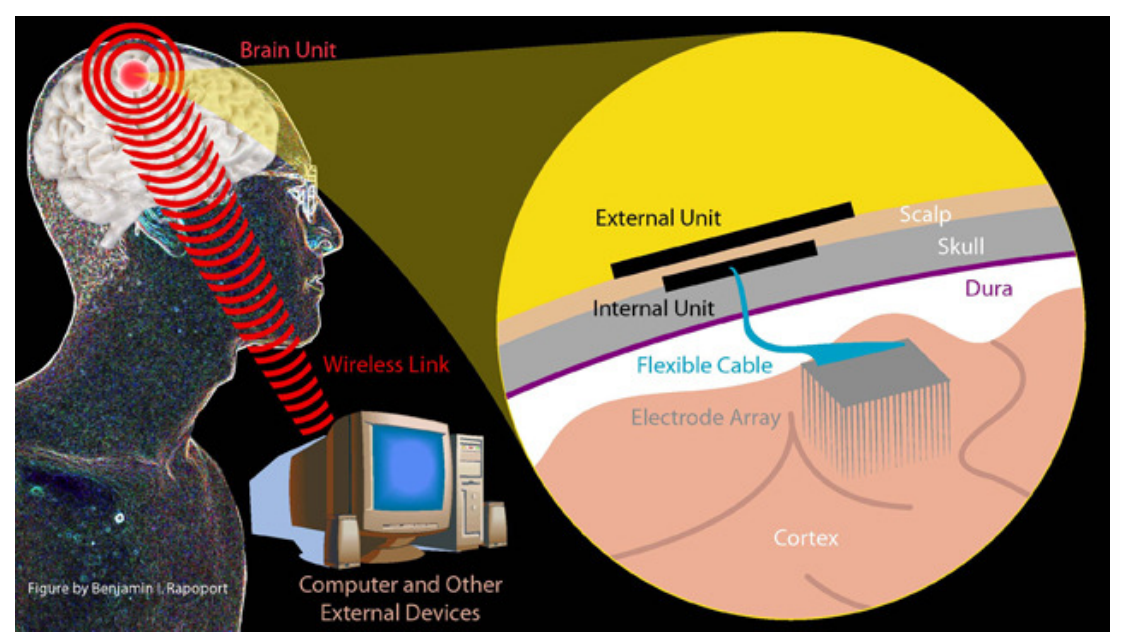

Figura 1.6: Imagem extraída do site do pesquisador Benjamin I. Rapoport (benjaminirapoport.com) 


\subsubsection{Interfaces que estimulam o sistema nervoso}

A ideia de empregar tecnologia para compensar deficiências ou doenças neurológicas é antiga. No entanto, foi o desenvolvimento da microeletrônica que permitiu a concretização de algumas aplicações consideradas bem sucedidas, atualmente. O princípio básico desses sistemas é a manipulação artificial do sistema nervoso biológico, utilizando-se sinais elétricos induzidos externamente, com o objetivo de emular funções sensoriais, motoras ou cognitivas.

A primeira e mais bem sucedida aplicação dessa ideia, de que se tem registro, é o implante coclear que teve início com o trabalho dos cientistas William House e Jack Urban nos anos 1960 (HOUSE; URBAN, 1973). Os modernos implantes cocleares multicanal converteram-se, atualmente, em um método clinicamente aceito para recuperação da audição.

Os implantes visuais apresentam uma complexidade muito maior do que aquela dos implantes cocleares, sobretudo porque requerem mais canais de estimulação. Enquanto os implantes cocleares funcionam adequadamente com 20 canais, os implantes visuais necessitam de centenas a milhares de canais. No âmbito da pesquisa, por exemplo, em 2013 foi anunciada a existência de um implante retinal de 512 canais (MONGE et al., 2013).

$\mathrm{Na}$ configuração mais encontrada na literatura de implantes visuais, uma câmera colocada sobre óculos é conectada a um processador de imagens. Como resultado do processamento das imagens, são enviados dados através de uma conexão sem fio para um chip implantado dentro do paciente. Este chip decodifica os sinais recebidos do processador de imagens e gera pulsos de corrente que são aplicados em eletrodos colocados na superfície da retina.

A companhia Second Sight, localizada na Califórnia, produz o implante retinal Argus que visa à melhora da visão em pacientes que sofrem de uma doença hereditária denominada retinite pigmentosa. Em março de 2011, a versão Argus II desse sistema recebeu aprovação para uso clínico e comercial na União Europeia. Em 2013, recebeu autorização da Food and Drug Administration (FDA) para uso clínico nos Estados Unidos. O sistema possui 60 canais de estimulação e custa aproximadamente US\$ 100.000 . 


\subsubsection{Interfaces neurais bidirecionais}

Dentre os exemplos de interfaces neurais bidirecionais, destacam-se os sistemas que permitem tratar a epilepsia. Mediante o registro de sinais neurais, pode-se antecipar a ocorrência de um evento epiléptico, o qual pode ser evitado através da aplicação de estímulos elétricos no sistema nervoso (SALAM; NGUYEN; SAWAN, 2010). Progressos em direção ao desenvolvimento de sistemas com essa dupla capacidade têm sido obtidos no contexto da neurociência experimental (BLUM et al., 2007), (BROWN et al., 2008).

Próteses cognitivas existem somente no âmbito de pesquisa. Um exemplo importante desse tipo de implante neural é o trabalho do professor Theodore Berger da Universidade do Sul da Califórnia (BERGER et al., 2012). O professor Berger e sua equipe desenvolveram uma prótese cognitiva projetada para recuperar a capacidade de formação de memórias de longo prazo, a qual é tipicamente perdida após um dano no hipocampo.

\subsubsection{Rumo da pesquisa em interfaces neurais implantáveis}

Através da revisão do estado da arte identificaram-se os seguintes interesses que norteiam o rumo da pesquisa em interfaces neurais implantáveis:

- Interesse em aumentar o número de eletrodos. Exemplo: Miguel Nicolelis relatou um experimento utilizando 768 micro eletrodos implantados em um macaco (Revista Science Translation Medicine, Novembro de 2013) (IFFT et al., 2013). Em 2014, o próprio Miguel Nicolelis relatou o implante de 1792 eletrodos (SCHWARTZ et al., 2014).

- O aumento do número de eletrodos traz como consequência o aumento na quantidade de informação a ser transmitida. Exemplo: 768 micro eletrodos $\times 25 \mathrm{~K}$ amostras $/ \mathrm{s} \times 10$ bits/amostras $=192 \mathrm{Mbits} / \mathrm{s}$.

- Quanto maior a taxa de transmissão torna-se mais difícil o projeto dos tranceptores considerando-se a limitação de dissipação de potência imposta pela integridade dos tecidos neurais: $227 \mu \mathrm{W} / \mathrm{mm}^{2}$ (KIM et al., 2007). Exemplo: em Outubro de 2013 Karim Abdelhalim, relatou o projeto de uma interface neural que pode 
transmitir até $10 \mathrm{Mbits} / \mathrm{s}$ (ABDELHALIM et al., 2013).

- Faz-se necessária a inclusão do processamento para redução da quantidade de informação a ser transmitida.

\subsection{Objetivos do projeto de doutorado}

$\mathrm{Na}$ revisão do estado da arte em interfaces neurais implantáveis, comprovou-se a existência de tendência a provê-las de capacidade de comunicação sem fio. Um dos fatores que limita a aplicação dessas interfaces sem fio em seres humanos é a elevada dissipação de potência causada por um requerimento de largura de banda na transmissão de grandes quantidades de informações. Além da dificuldade de se alimentar o circuito implantado a partir de uma unidade externa, existe um limite de dissipação de potência que não deve ser ultrapassado a fim de evitar danos aos tecidos neurais. Verificou-se também que um dos caminhos escolhidos para reduzir a potência dissipada é a adição de tarefas de pré-processamento dentro do circuito implantável. A inclusão desses estágios permite diminuir a taxa de transmissão necessária pela interface de comunicação sem fio e, dessa maneira, reduz-se a dissipação de potência. Os pré-processamentos que apresentam maior impacto na redução de potência são a detecção de impulsos elétricos e a separação de impulsos elétricos (spike sorting). Dependendo da aplicação, podem ser suficientes apenas um deles ou ambos.

Dado esse panorama sobre o rumo da pesquisa, escolheu-se, como projeto de doutorado, o desenvolvimento de um sistema de pré-processamento de impulsos elétricos neurais (spikes) que pode ser integrado dentro de uma interface neural implantável com capacidade de comunicação sem fio. Enunciam-se, a seguir, os objetivos gerais desta tese.

\subsubsection{Objetivos Gerais}

- Desenvolver um sistema de detecção de impulsos elétricos neurais em tecnologia CMOS a ser integrado em uma interface neural implantável sem fio;

- Desenvolver um sistema de classificação de impulsos elétricos neurais em tecnologia CMOS a ser integrado em uma interface neural implantável sem fio. 
Para facilitar a avaliação da relevância científica dos objetivos deste doutorado, elaborou-se a tabela 1.1 onde se encontra uma lista de publicações recentes que demonstram o esforço realizado pela comunidade científica em abordar problemas similares aos tratados nesta tese. É necessário salientar que essa lista é parcial e que a quantidade de artigos relacionados, encontrados na revisão do estado da arte, é muito maior; como se pode verificar na lista de referências bibliográficas no final deste texto.

Os artigos citados nessa tabela correspondem aos seguintes periódicos e congressos:

- IEEE Journal of Solid-State Circuits (JSSC)

- IEEE International Solid-State Circuits Conference (ISSCC)

- IEEE Transactions on Neural Systems and Rehabilitation Engineering (TNSRE)

- IEEE International Symposium on Circuits and Systems (ISCAS)

- IEEE Biomedical Circuits and Systems Conference (BioCAS) 
Tabela 1.1: Lista de publicações recentes que abordam os problemas de detecção e separação de impulsos elétricos neurais em interfaces neurais implantáveis

\begin{tabular}{|l|c|l|}
\hline Referência & $\begin{array}{c}\text { Periódico/ } \\
\text { Congresso }\end{array}$ & Descrição \\
\hline (CHAE et al., 2008) & JSSC & $\begin{array}{l}\text { Sistema de registro neural com extração } \\
\text { de características e spike sorting. }\end{array}$ \\
\hline (SODAGAR et al., 2009) & $\begin{array}{l}\text { Sistema de registro neural com deteção } \\
\text { de impulsos elétricos. }\end{array}$ \\
\hline (KARKARE; GIBSON; MARKOVIC, 2013) & JSSC & $\begin{array}{l}\text { Processador digital que realiza o Spike } \\
\text { Sorting }\end{array}$ \\
\hline (DATTA-CHAUDHURI et al., 2014) & BioCAS & $\begin{array}{l}\text { Sistema de detecção de spikes baseado } \\
\text { no operador não linear de energia (NEO). }\end{array}$ \\
\hline (YANG; MASON, 2014) & ISCAS & $\begin{array}{l}\text { Sistema de detecção de spikes baseado } \\
\text { no operador não linear de energia (NEO). }\end{array}$ \\
\hline (ZAMANI; DEMOSTHENOUS, 2014) & TNSRE & $\begin{array}{l}\text { Sistema de extração de características } \\
\text { baseadas nas derivadas discretas do si- } \\
\text { nal. }\end{array}$ \\
\hline (DO; YEO, 2014) & TSCAS & $\begin{array}{l}\text { Sistema de detecção de spikes baseado } \\
\text { no operador não linear de energia (NEO). }\end{array}$ \\
\hline (DRAGAS et al., 2015) & $\begin{array}{l}\text { Otimização do uso de recursos de hard- } \\
\text { ware na implementação do algoritmo de } \\
\text { template matching aplicado ao spike sor- } \\
\text { ting. }\end{array}$ \\
\hline (ZEINOLABEDIN et al., 2015) & ISCAS & $\begin{array}{l}\text { Sistema híbrido de detecção baseado no } \\
\text { operador NEO e no método de aplicação } \\
\text { simples de limiar. }\end{array}$ \\
\hline
\end{tabular}

\subsubsection{Objetivos Específicos}

$\mathrm{Na}$ subseção anterior, tornou-se claro que o presente trabalho de doutorado aborda os problemas de detecção e separação de impulsos elétricos neurais em interfaces neurais implantáveis sem fio. Na presente subseção, mencionam-se, de maneira mais específica, os caminhos escolhidos para abordar os problemas de processamento de sinais neurais.

No caso da detecção de impulsos elétricos neurais, um dos aspectos do estado da arte é a aplicação de um processamento de pré- ênfase ao sinal neural. Esse processamento tem como propósito enfatizar a diferença entre impulsos elétricos neurais e o ruído de fundo. Como mostra a tabela 1.1, as publicações mais recentes relatam o uso do operador não linear de energia (NEO) como opção para a pré- ênfase do sinal em sistemas de detecção de impulsos elétricos neurais. Isso se deve à simplicidade desse operador que permite idealizar implementações com menos custo de hardware e energia. Dado esse panorama, propuseram-se os seguintes objetivos específicos 
para o desenvolvimento do sistema de detecção de impulsos elétricos neurais:

- Desenvolver um processador que executa a operação NEO;

- Projetar um comparador que processe a saída do circuito NEO;

- Empregar processamento analógico em modo corrente para simplificar o hardware necessário para implementar as operações.

No caso da classificação de impulsos elétricos, estabeleceram-se os seguintes objetivos específicos:

- Desenvolver um classificador de spikes baseado no formato dos mesmos;

- Implementar um circuito que calcule a distância de Manhattan entre dois conjuntos de amostras;

- Empregar uma estratégia de processamento em modo tempo que permita reduzir a complexidade em termos de hardware e consumo de potência. 


\subsection{Estratégia de avaliação dos sistemas desenvolvi- dos neste trabalho}

A presente tese discute problemas sobre processamento dos sinais neurais. Para avaliar os resultados desses processamentos, é necessário conhecer, a priori, as características dos sinais que são processados. Esse conhecimento prévio das características dos sinais é possível através do emprego de sinais sintéticos (GIBSON; JUDY; MARKOVIĆ, 2008). A fim de entender melhor essa ideia, apresenta-se, a seguir, uma discussão sobre a maneira como foram avaliados os sistemas desenvolvidos neste doutorado.

No caso do sistema de detecção de spikes, o desempenho pode ser avaliado se conhecermos os instantes em que, de fato, eles acontecem. Dessa maneira, podemos decidir se o instante de ocorrência de um spike, indicado por nosso sistema, corresponde a um spike verdadeiro (true positive), ou se corresponde a um evento de ruído que foi confundido com spike (false positive). Já para o caso do sistema de separação de spikes, além dos instantes de ocorrência, precisamos conhecer a procedência (neurônio gerador) de cada um deles. Existem várias maneiras de dispor de um sinal neural conhecendo os instantes de ocorrência de spikes e sua procedência.

Uma delas consiste em usar registros intracelulares e extracelulares simultâneos similares aos relatados em (HENZE et al., 2000). Porém, além da existência de poucos bancos de dados com essas características e do seu difícil acesso, vários pesquisadores concordam que o escopo de avaliação dos sistemas de processamento de sinais neurais através desses bancos de dados seria muito limitado (GIBSON; JUDY; MARKOVIĆ, 2008), (HARRIS et al., 2000), (PARASKEVOPOULOU; BARSAKCIOGLU; SABERI, 2013), (NAVAJAS et al., 2014). Além disso, para cada par de sinais intracelular/extracelular - apesar do registro de atividades de vários neurônios por um eletrodo extracelular - apenas um deles é confirmado pelo registro intracelular.

Outra maneira de se obter sinais neurais para testes dos sistemas é registrar os sinais extracelularmente e confiar a um especialista a tarefa de identificar os spikes por inspeção visual. Neste caso, levar-se-ia muito tempo para gerar um banco de dados suficientemente grande. Por outro lado, existe a desconfiança de alguns pesquisadores sobre a validade desses tipos de sinais para fins de avaliação dos sistemas de processamento de sinais neurais (HARRIS et al., 2000), (WOOD et al., 2004). Por exemplo, o estudo relatado em (HARRIS et al., 2000) mostrou casos nos quais o desem- 
penho de um operador humano foi inferior ao de uma ferramenta semiautomática de classificação.

Por último, existe a possibilidade de emprego de dados sintéticos. Neste caso, não há problemas de tamanho do banco de dados a ser utilizado. Ademais, é possível conhecer exatamente os instantes em que acontecem os spikes e sua procedência e introduzir níveis conhecidos de ruído. Por esse motivo, assim como na maioria dos trabalhos similares a este, recorreu-se aos dados sintéticos para avaliar o desempenho dos sistemas desenvolvidos nesta tese. Algumas simulações foram realizadas com sinais reais apenas como finalidade de ilustração. 


\subsubsection{Banco de dados escolhido para a avaliação dos sistemas desenvolvidos nesta tese}

A seguir, procede-se à apresentação do banco de dados, com descrição de sua estrutura e características, e comentam-se as razões pelas quais foi escolhido.

Através do estudo do estado da arte, encontraram-se alguns bancos de dados de sinais neurais sintéticos que são recorrentemente utilizados nas pesquisas relacionadas ao processamento de sinais neurais. A tabela 1.2 lista os bancos de dados que foram mais frequentemente encontrados nos artigos revisados.

Tabela 1.2: Lista de bancos de dados de sinais neurais encontrados no estudo do estado da arte

\begin{tabular}{|l|c|l|}
\hline $\begin{array}{l}\text { Referência } \\
\text { (QUIROGA; NADASDY; BEN-SHAUL, 2004) }\end{array}$ & $\begin{array}{c}\text { Tipo } \\
\text { Sintético }\end{array}$ & $\begin{array}{l}\text { Breve Descrição } \\
\text { Baseado em spikes reais. Ruído gerado a } \\
\text { partir da soma randômica de spikes reais. }\end{array}$ \\
\hline $\begin{array}{l}\text { (RUTISHAUSER; SCHUMAN; MAMELAK, 2006) } \\
\text { Sintético }\end{array}$ & $\begin{array}{l}\text { Baseado em spikes reais. Ruído gerado a } \\
\text { partir da soma randômica de spikes reais. }\end{array}$ \\
\hline
\end{tabular}

Entre esses bancos de dados, o primeiro (QUIROGA; NADASDY; BEN-SHAUL, 2004) foi encontrado mais vezes nos artigos que tratam de problemas similares aos abordados nesta tese (GIBSON; JUDY; MARKOVIĆ, 2008). Esse banco de dados está disponível junto a uma ferramenta de spike sorting denominada Waveclus. Seus sinais foram gerados a partir de um conjunto de 594 spikes reais.

É possível gerar novos sinais sintéticos por meio da ferramenta disponibilizada junto a esse banco de dados. Escolhendo-se o número de neurônios, a taxa de disparos e o desvio padrão do ruído; a ferramenta gera uma forma de onda com os spikes escolhidos, colocados randomicamente no tempo, e respeitando-se um período refratário e a taxa de disparos. O ruído é gerado mediante uma escolha aleatória de spikes (do conjunto de 594 spikes disponíveis). Essa maneira de gerar ruído é mais biologicamente plausível do que a simples inclusão de ruído branco Gaussiano. 


\subsection{Estrutura da tese}

A seguir detalha-se a estrutura da tese através de uma descrição do conteúdo de cada capítulo.

\section{Capítulo 2:}

- Explicação do funcionamento interno de uma interface neural implantável. Exemplos de sistemas desse tipo relatados na literatura estudada;

- Explicação do funcionamento de um amplificador neural e principais desafios: redução do consumo de potência, redução do ruído gerado pelos dispositivos, eliminação do offset introduzido pelos eletrodos. Soluções encontradas na literatura;

- Descrição das arquiteturas mais utilizadas nas interfaces neurais implantáveis. Características requeridas pela aplicação e resultados relatados nos artigos estudados: número de bits, frequência de amostragem, consumo de potência, área ocupada, tensão de alimentação;

- Exemplos de sistemas implantáveis onde, além de se fazerem a leitura e condicionamento dos sinais, realiza-se algum processamento antes de transmitir os resultados através da comunicação sem fio;

- Revisão teórica do funcionamento de um acoplamento indutivo. Considerações para o projeto de um enlace indutivo de uma interface neural implantável. Transmissão de informação através do acoplamento indutivo.

\section{Capítulo 3:}

- Revisão teórica sobre o processamento de detecção de impulsos elétricos neurais;

- Revisão do estado da arte em métodos de detecção empregados em circuitos integrados implantáveis;

- Revisão do estado da arte em métodos para seleção do limiar de comparação;

- Explicação da necessidade do estágio de pré-ênfase e revisão do estado da arte; 
- Revisão teórica sobre o operador não linear de energia (NEO) também chamado de operador Teager;

- Explicação da importância do spike sorting nos contextos de BCl (Brain Computer Interface) e neuropróteses motoras.

\section{Capítulo 4}

- Explicar como se obtém a expressão do NEO utilizando como operador básico um elevador ao quadrado;

- Mencionar a vantagem, em termos de implementação em hardware, de trabalhar com o valor absoluto das amostras analógicas. Mostrar as simulações, feitas em Matlab, das aplicações do operador NEO ao sinal original e ao valor absoluto do sinal;

- Explicar o funcionamento do circuito conversor tensão-corrente projetado;

- Projeto e análise do circuito elevador ao quadrado;

- Projeto e análise do circuito que realiza a operação NEO;

- Simulações com os sinais do banco de dados selecionado;

- Análise dos resultados de simulação e discussão final sobre o sistema projetado.

\section{Capítulo 5}

- Adaptação da estratégia apresentada em 2013 por Sara Gibson (KARKARE; GIBSON; MARKOVIC, 2013) a um esquema baseado em atrasos entre pulsos digitais;

- Explicação da estratégia proposta para colocar numa mesma janela de tempo os pulsos digitais representantes das amostras analógicas;

- Explicação do funcionamento do circuito conversor tensão-tempo. Justificativa da escolha da topologia do circuito;

- Explicação da estratégia proposta para o cômputo da distância de Manhattan baseada no uso de atrasos entre pulsos digitais. Apresentação de simulações mostrando o desempenho; 
- Simulações com os sinais do banco de dados selecionado;

- Análise dos resultados de simulação e discussão final sobre o sistema projetado.

\section{Capítulo 6}

Finalmente, no capítulo 6 apresentam-se as conclusões deste trabalho bem como propostas para trabalhos futuros. 


\section{Sistemas implantáveis de leitura, processamento e transmissão sem fio de sinais neurais extracelulares: descrição e o estado da arte}

Uma das conclusões do capítulo 1 foi que há interesse em dispensar o uso de cabos que atravessam o crânio. Neste capítulo veremos vários exemplos de tentativas de eliminar esses cabos através do desenvolvimento de interfaces neurais implantáveis sem fio. A motivação para esse interesse é tanto na área de desenvolvimento de próteses motoras quanto na área de estudo de redes neurais biológica em animais. Além de fornecer conforto e liberdade de movimento aos pacientes, os sistemas sem fios reduzem o risco de infecções. Para o caso de estudos em animais é interessante para os pesquisadores registrar os sinais neurais enquanto $O$ animal se movimenta livremente no seu ambiente natural (SANTHANAM et al., 2007).

Este capítulo aborda os sistemas implantáveis que realizam o registro de sinais neurais extracelulares mediante eletrodos implantados no cérebro, processam tais sinais e transmitem através de um canal de comunicação sem fio informação relevante para aplicações de $\mathrm{BCl}$ ou de neuropróteses motoras. O capítulo inicia com uma revisão de sistemas desse tipo encontrados nos artigos estudados. Posteriormente, descrevem-se com detalhe as diversas partes que compõem esses sistemas e se expõem exemplos de implementações.

$\mathrm{Na}$ figura 2.1 representa-se de maneira simplificada um canal de leitura, processamento e transmissão de sinais neurais extracelulares. O sinal medido pelo eletrodo implantado passa por um estágio de condicionamento que consiste na amplificação e filtragem no domínio da frequência. Posteriormente o sinal é convertido ao domínio 
digital para ser processado. Finalmente o sinal é modulado e transmitido através do canal de comunicação sem fio.

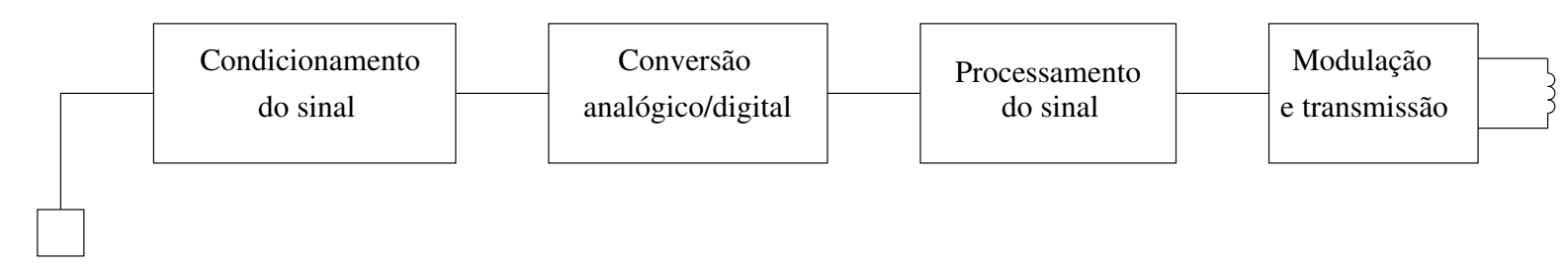

Eletrodo

Figura 2.1: Representação simplificada de um canal de leitura, processamento e transmissão de sinais neurais extracelulares

Na maioria dos casos as interfaces neurais implantáveis registram sinais não apenas de um eletrodo mas de vários deles. Então, para cada eletrodo precisar-se-ia de um canal como o representado na figura 2.1, porém alguns do componentes mostrados nesse diagrama simplificado podem ser compartilhados por vários canais. $\mathrm{Na}$ figura 2.2 representa-se um sistema multicanal onde alguns componentes são compartilhados.

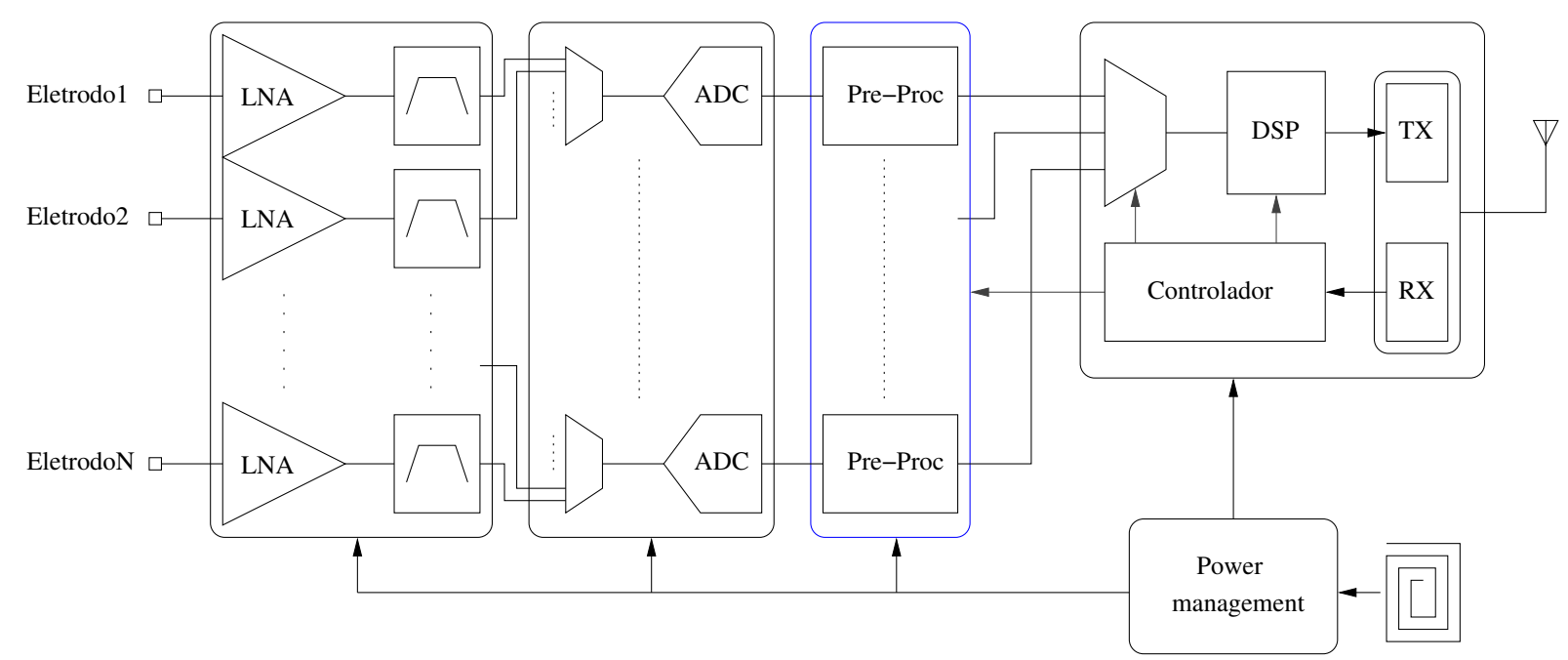

Figura 2.2: Diagrama de blocos de uma interface neural implantável multicanal

Como se observa na figura 2.2 um único conversor analógico-digital (ADC) pode ser compartilhado por vários canais através de um multiplexador analógico (MUX). Quanto maior o número de canais que compartilham um ADC menor a área. Porém, ao aumentar o número de canais por ADC aumenta a capacitância de saída do multiplexador e com isso aumenta a corrente necessária para estabelecer o sinal no nó de saída do MUX. Essa corrente é fornecida por um buffer prévio ao MUX. Por tanto existe um compromisso entre área e consumo de potência ao escolher o número de canais que compartilham um único ADC. Ao aumentar o número de canais por ADC também 
aumenta a frequência de operação de ADC o que aumenta o consumo de potência. Um estudo analítico apresentado em (CHAE; LIU; SIVAPRAKASAM, 2008) levou à conclusão que o número de canais por ADC que minimiza o produto área $\times$ consumo é 16. Vários trabalhos tem adotado esse número com referência para a implementação de sistemas implantáveis (CHAE et al., 2009), (TAO; RUSU, 2015).

\subsection{Sistemas implantáveis de registro neural sem fio - o estado da arte}

Atualmente existem vários grupos de pesquisa que tem desenvolvido protótipos de sistemas implantáveis de registro neural sem fio. A seguir apresenta-se uma série de protótipos que tem sido testados em animais. O objetivo da maioria desses grupos de pesquisa é desenvolver o sistema de maneira que possa ser implantado em um ser humano. Até a data de apresentação deste documento o autor não encontrou registros de aplicações de interfaces implantáveis sem fio em seres humanos. Nos exemplos que serão vistos nesta seção analisaremos cuidadosamente os detalhes da avaliação experimental dos sistemas.

Em 1998, pesquisadores da Universidade de Michigan, Ann Arbor, publicaram no Journal of Solid-State Circuits um artigo relatando o desenvolvimento de um sistema de registro neural wireless (AKIN; NAJAFI; BRADLEY, 1998). O sistema foi projetado para registrar sinais de axônios do sistema nervoso periférico através de eletrodos específicos conhecidos como Sieve Electrodes. Sinais de 32 pontos de registro são precondicionados mediante amplificadores que possuem faixa linear de entrada de $\pm 500 \mu \mathrm{V}$ e largura de banda entre $100 \mathrm{~Hz}$ e 3,1 KHz. Dos 32 sinais, 2 podem ser selecionados, multiplexados no domínio temporal e aplicados a um conversor analógico digital de 8 bits que consome menos de $2 \mathrm{~mW}$. O diagrama de blocos é apresentado na figura 2.3. O consumo total é de $90 \mathrm{~mW}$, dos quais $80 \mathrm{~mW}$ correspondem à interface RF. 


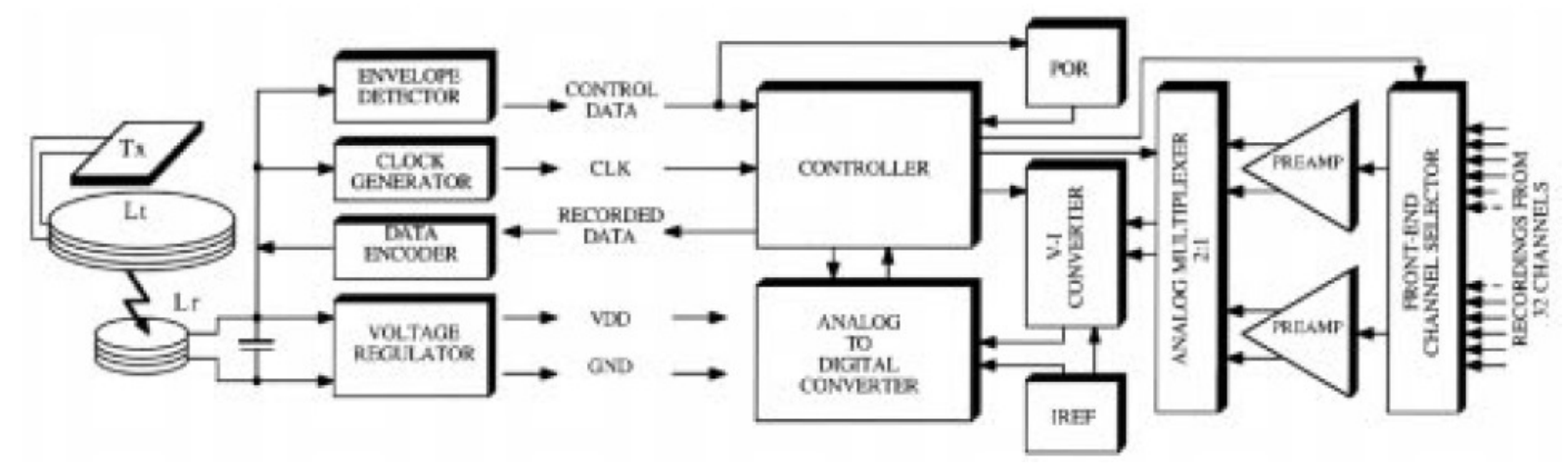

Figura 2.3: Diagrama de blocos do sistema apresentado em (AKIN; NAJAFI; BRADLEY, 1998). Figura extraída do artigo (AKIN; NAJAFI; BRADLEY, 1998).

Um dos grupos que vem trabalhando em versões sem fio de interfaces neurais implantáveis é o Grupo de Microssistemas Integrados Sem Fio da Universidade de Michigan, liderado pelo professor Kensall Wise. Antes de trabalhar em versões sem fio dos protótipos desenvolveram vários circuitos necessários numa interface neural. Um trabalho desse grupo que é muito referenciado na literatura sobre implantes neurais é o apresentado em (OLSSON-III; WISE, 2005). Trata-se de um sistema composto por 4 conjuntos de eletrodos e um ASIC (Application Specific Integrated Circuit) para compressão de dados. Cada um dos 4 conjuntos é denominado Active Neural Probe ou sonda neural ativa. Em cada da sonda existem 64 pontos de medição integrados num mesmo substrato junto com circuitos que permitem a amplificação, filtragem e multiplexação dos sinais analógicos. A figura 2.4, que foi extraída do artigo (OLSSONIII; WISE, 2005), ilustra uma dessas quatro sondas ativas.

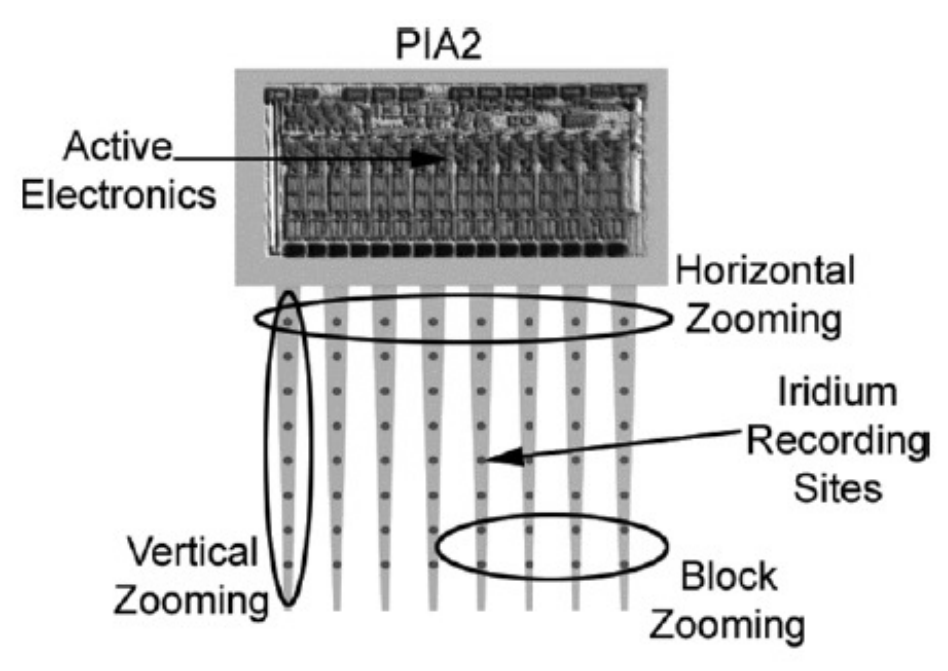

Figura 2.4: Sonda ativa utilizada no sistema de Olsson e Wise. Imagem extraída de (OLSSON-III; WISE, 2005) 
As saídas das sondas ativas contém sinais analógicos de 8 canais multiplexados no tempo. Os 8 canais são escolhidos entre os 64 disponíveis através de outros multiplexadores analógicos. Essas saídas são enviadas ao ASIC de compressão de dados, como mostrado no diagrama do sistema mostrado na figura 2.5.

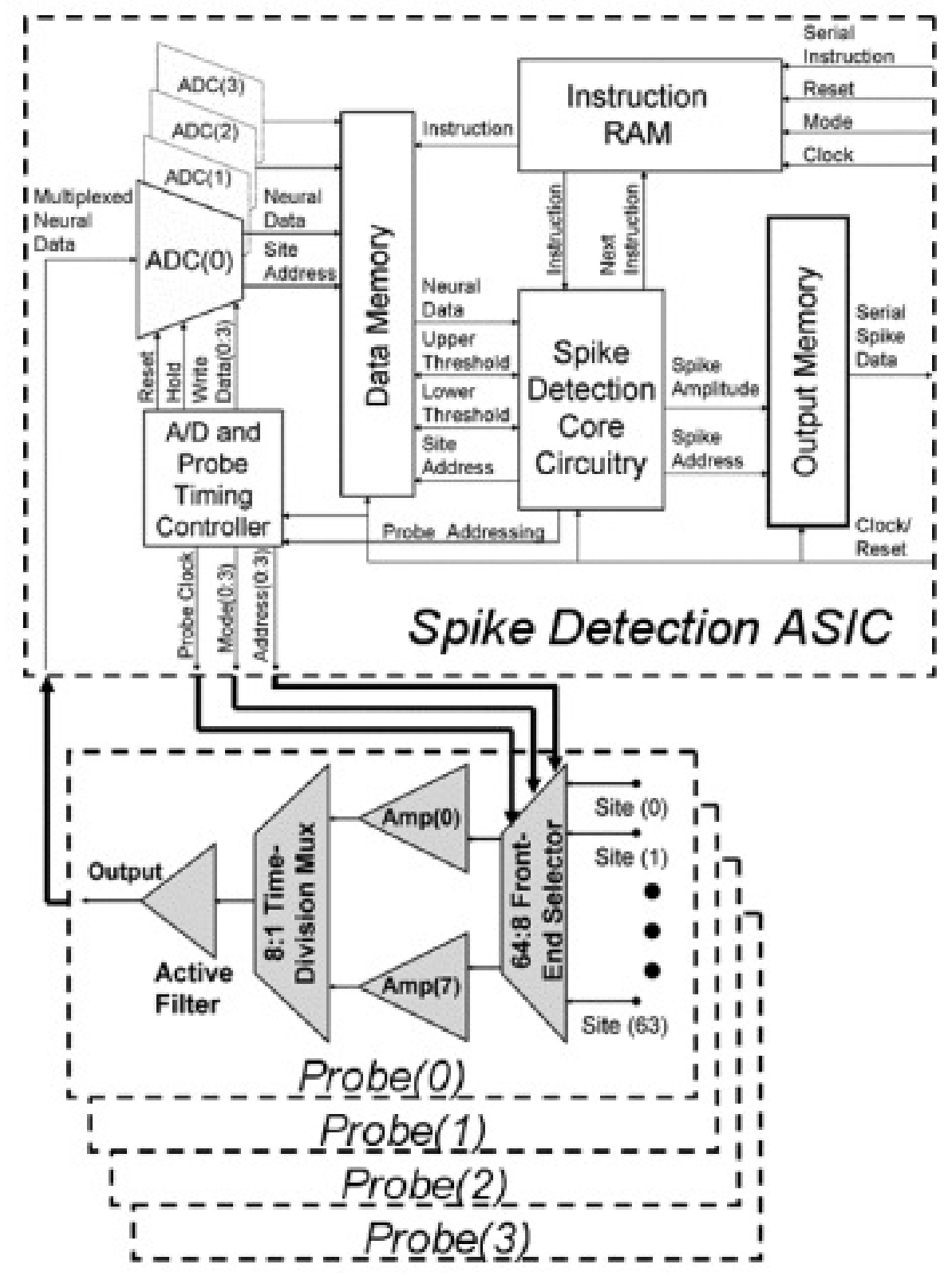

Figura 2.5: Diagrama de blocos do sistema apresentado em (OLSSON-III; WISE, 2005). Imagem extraída de (OLSSON-III; WISE, 2005).

É interessante que a estratégia de compressão utilizada por este sistema, a detecção de spikes, continua sendo hoje uma alternativa importante. Como mencionado anteriormente este sistema é um dos mais referenciados na literatura (ademais, o trabalho foi publicado num dos periódicos mais importantes na área da microeletrônica: Journal of Solid States Circuits). 
Um dos primeiros protótipos sem fio deste grupo foi exposto na IEEE Solid-State Sensors, Actuators and Mycrosystems Conference em Junho de 2007 (SODAGAR et al., 2007). Na figura 2.6 observa-se o diagrama de blocos desse sistema junto com os periféricos que o complementam. O sistema está composto por 7 chips: 4 de amplificadores e multiplexadores, 2 processadores de sinais neurais e 1 de telemetria bidirecional.

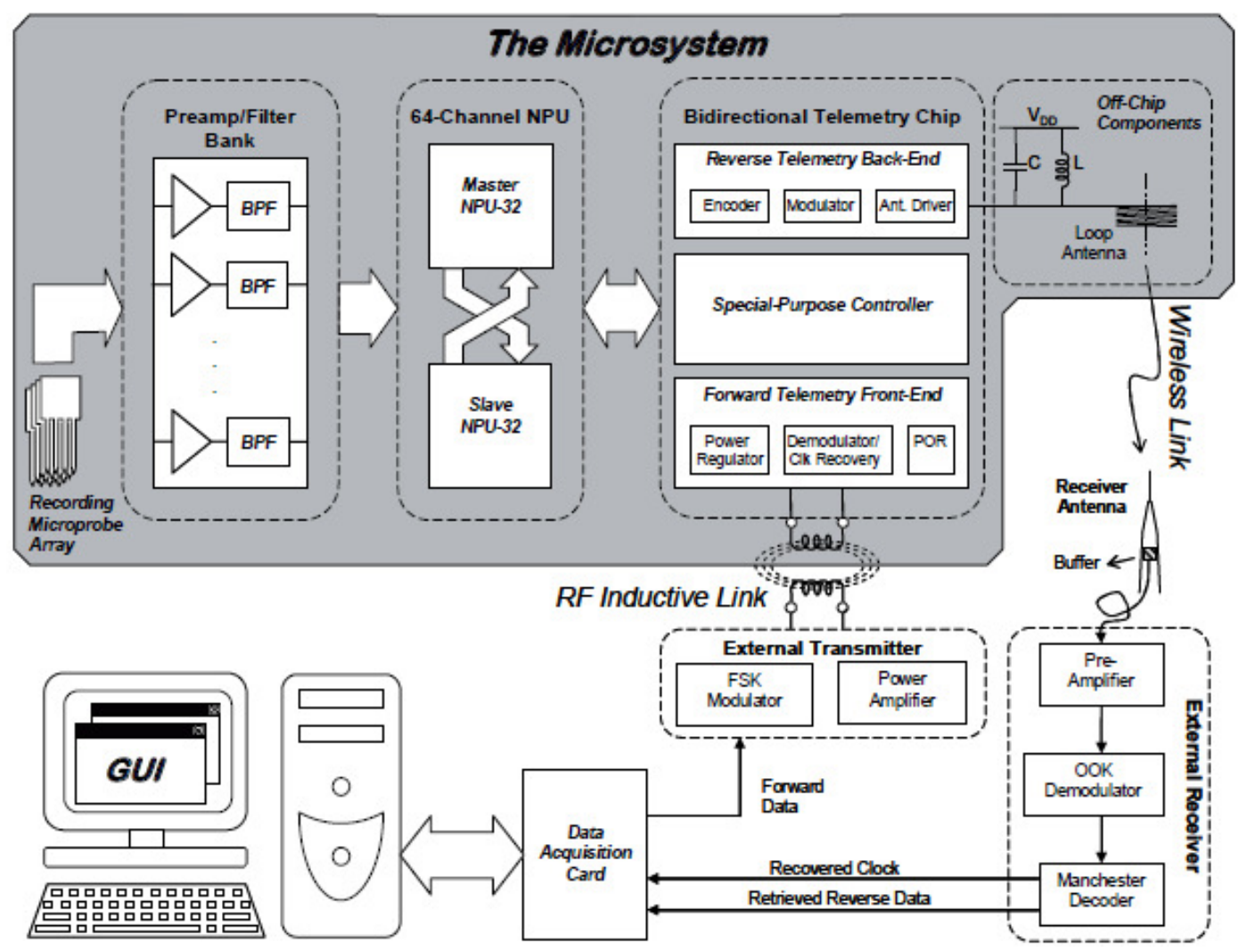

Figura 2.6: Diagrama de blocos internos e periféricos externos do sistema apresentado em junho 2007 pelo Center for Wireless Integrated MicroSystems (WIMS) da universidade de Michigan. Imagem extraída de (SODAGAR et al., 2007). NPU (Neural Processor Unit), BPF (Band Pass Filter) e POR(Power On Reset).

Esse sistema processa sinais de 64 eletrodos e possui duas interfaces sem fio, uma para recepção de comandos de configuração ( $R F$ Inductive Link) e outra para transmissão dos sinais neurais registrados (Wireless Link). O sistema contém um detector de spikes e comunica através do canal Wireless a ocorrência do spike e um identificador do canal que o registrou. Também existe a opção de selecionar um eletrodo, digitalizar o sinal medido em 8 bits e transmitir o resultado. Na modalidade de registro contínuo do sinal de um eletrodo, o sistema consome $14.4 \mathrm{~mW}$, sendo 62.5 KSamples/s a taxa de amostragem e 2 Mbps a taxa de transmissão. A capacidade de comunicação sem fio do sistema foi verificada mediante um monitoramento durante 
30 dias dos sinais medidos por dois eletrodos implantado no córtex auditivo de dois porcos. É importante salientar que apenas os eletrodos foram implantados, pois o chip ficou numa placa de circuito impresso localizada na parte externa do escalpo.

O processador neural (NPU) tinha sido apresentado previamente no IEEE Biomedical Circuits And Systems Conference de 2006 (SODAGAR; WISE; NAJAFI, 2006).

Na maioria de sistemas divulgados até 2007 pode-se transmitir simultaneamente de maneira sem fio os sinais de apenas um subconjunto dos canais disponíveis. Por exemplo no sistema de (SODAGAR; WISE; NAJAFI, 2006) mesmo tendo disponíveis 64 canais de registro é possível transmitir simultaneamente todas as amostras coletadas de apenas um eletrodo. Esse panorama começou a mudar a partir de 2008 quando por exemplo a equipe do professor Wentai Liu da Universidade de Califórnia Santa Cruz desenvolveu um sistema de 128 canais capaz de transmitir simultaneamente as amostras de todos os canais, para o qual a interface wireless precisou ter uma capacidade de transmissão de 90 Mbs (CHAE et al., 2008), (CHAE et al., 2009). O diagrama de blocos desse sistema está representado na figura 2.7 que foi extraída do artigo (CHAE et al., 2009).

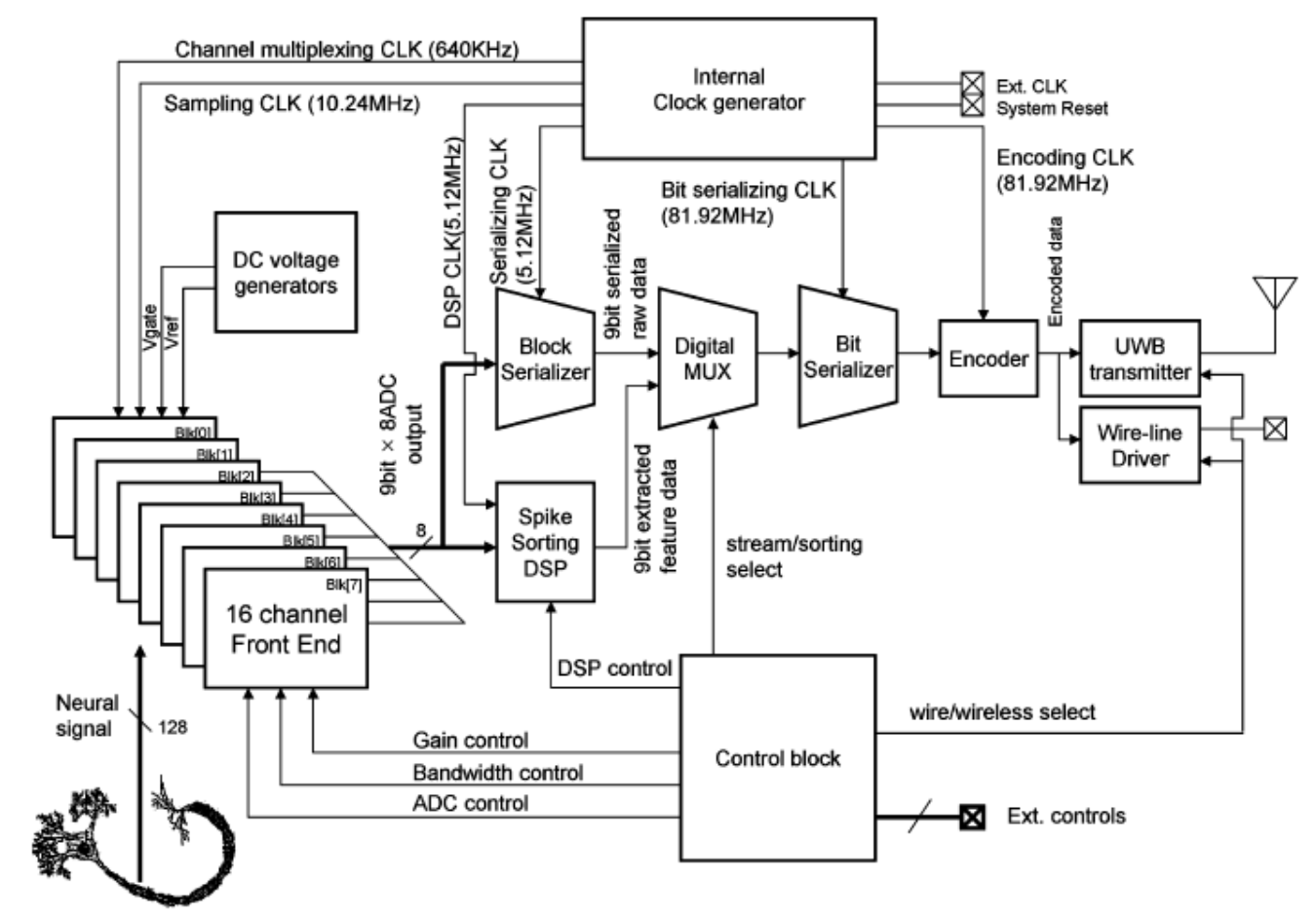

Figura 2.7: Diagrama de blocos do sistema desenvolvido pela equipe do professor Wentai Liu da Universidade de Califórnia Santa Cruz. Imagem extraída do artigo (CHAE et al., 2009) 
O consumo total desse sistema é $6 \mathrm{~mW}$ sendo que o transmissor consome 1.6 $\mathrm{mW}$. Uma estratégia para redução do consumo de potências utilizada neste sistema é a ativação sequencial de circuitos. Como as amostras são multiplexadas temporariamente não é necessário manter ativados todos os circuitos de todos os canais. Para atingir uma taxa de transmissão necessária para enviar sinais dos 128 canais o sistema possui um transmissor baseado em IR UWB (Impulse Radio Ultra Wide Band).

Esse sistema possui um detector de impulsos elétricos baseado no operador não linear de energia NEO e extrai características dos impulsos elétricos detectados. As três características escolhidas pelos desenvolvedores desse sistema são a amplitude máxima do spike, o valor mínimo da primeira derivada no intervalo do spike e o valor máximo dessa derivada.

Nota-se claramente que com o passar dos anos as características dos sistemas publicados nas conferências e periódicos vão melhorando em termos de quantidade de canais e consumo de potência.

Em Outubro 2013, no IEEE Transactions on Circuits and Systems-I: Regular Papers foi publicado um artigo que mostra o desenvolvimento de um sistema de 100 canais que consume $1.16 \mathrm{~mW}$ (ZOU et al., 2013). Na figura 2.8 ilustram-se os componentes implantáveis desse sistema. O circuito integrado IC1 realiza o condicionamento dos sinais (amplificação, filtragem no domínio da frequência e conversão analógicodigital), o circuito integrado IC2 realiza o processamento, modulação e transmissão dos sinais, a bobina (Coil) é usada para receber energia através de acoplamento indutivo e finalmente a antena é utilizada para a transferência de dados via comunicação RF. Essa divisão entre condicionamento dos sinais e processamento é similar à encontrada no trabalho de Olsson e Wise (OLSSON-III; WISE, 2005) que foi discutido anteriormente, e também se encontra em vários outros trabalhos estudados na literatura. Uma diferença entre o trabalho de Zou e o trabalho de Olsson é que no primeiro caso o condicionamento inclui também a conversão ao domínio digital. O número efetivo de bits (ENOB) é 8.5, similar a outro trabalhos. No trabalho de Olsson o conversor era um componente interno do ASIC de processamento.

A faixa de frequência do filtro pode ser configurada para poder registrar spikes ou para registrar atividade elétrica coletiva de baixa frequência (Field Potentials). Foram realizados experimentos em ratos inconscientes. 


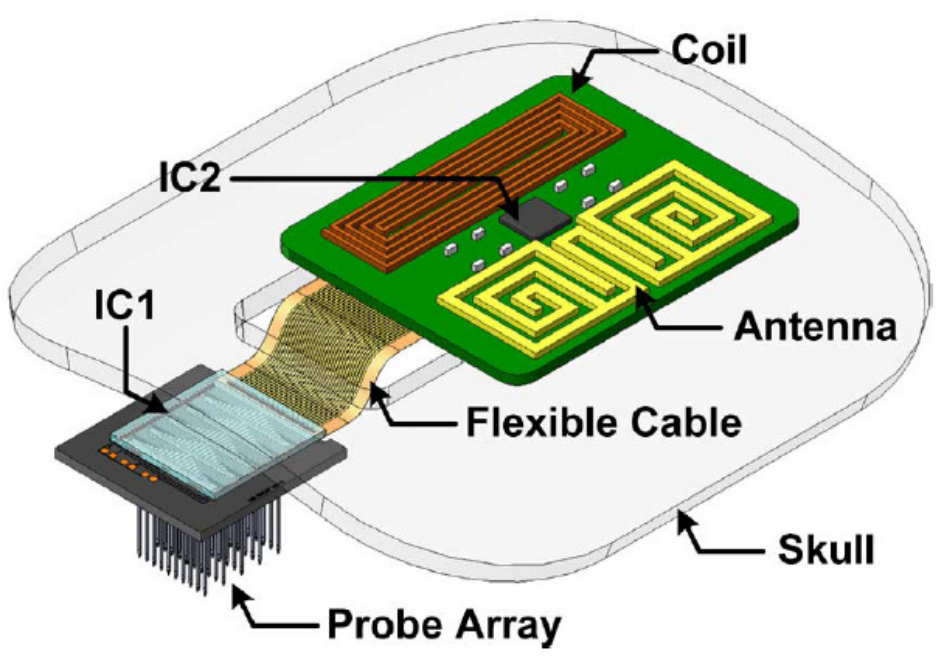

Figura 2.8: Ilustração do sistema apresentado em (ZOU et al., 2013). Imagem extraída do artigo (ZOU et al., 2013).

A figura 2.9 mostra o diagrama de blocos desse sistema. Notam-se algumas características em comum com outros sistemas discutidos neste capítulo como o compartilhamento de um conversor analógico-digital entre vários canais. Como mencionado anteriormente a carga no nó de saída do multiplexador devido às difusões dos transistores das chaves impõe restrições nos buffers que estão antes do multiplexador.

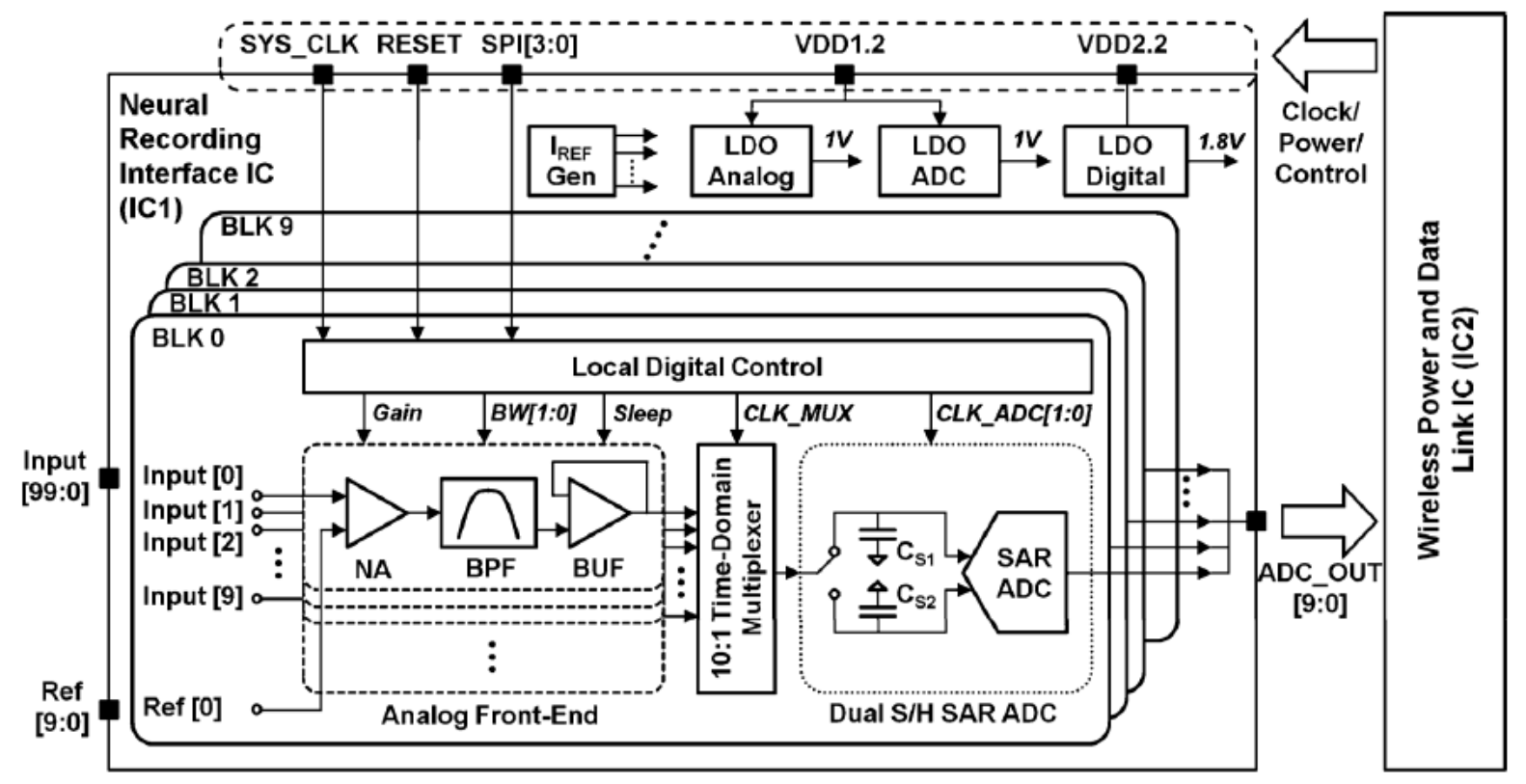

Figura 2.9: Diagrama de blocos do sistema apresentado em (ZOU et al., 2013). Imagem extraída do artigo (ZOU et al., 2013). 
Em 2014 uma equipe liderada pelo professor Jan Rabaey publicou um artigo na ISSCC (International Solid-State Circuits Conference), a conferência mais importante na área da microeletrônica, que aborda o desenvolvimento de um sistema de medição de sinais de eletrocorticografia (ECoG) (MULLER et al., 2014). Este tipo de sinais são registrados na superfície do cérebro o que representa um procedimento menos invasivo que aquele empregado para medir os sinais neurais extracelulares. Os autores apontam como desvantagem do registro com microeletrodos implantados que penetram o córtex que a cicatrização produzida muda a qualidade dos sinais com o passar do tempo. Esse mesmo argumento é encontrado na análise de Gerwin Schalk apresentada em (SCHALK, 2010).

$\mathrm{Na}$ figura 2.10 ilustra-se o sistema. Um total de 64 eletrodos podem ser monitorados consumindo apenas $225 \mu \mathrm{W}$. Notam-se como características inovadoras em relação aos trabalhos previamente discutidos o uso da técnica chopper para eliminação de offset e o uso da técnica de modulação de carga tipo Backscattering para transmissão sem fio de dados.

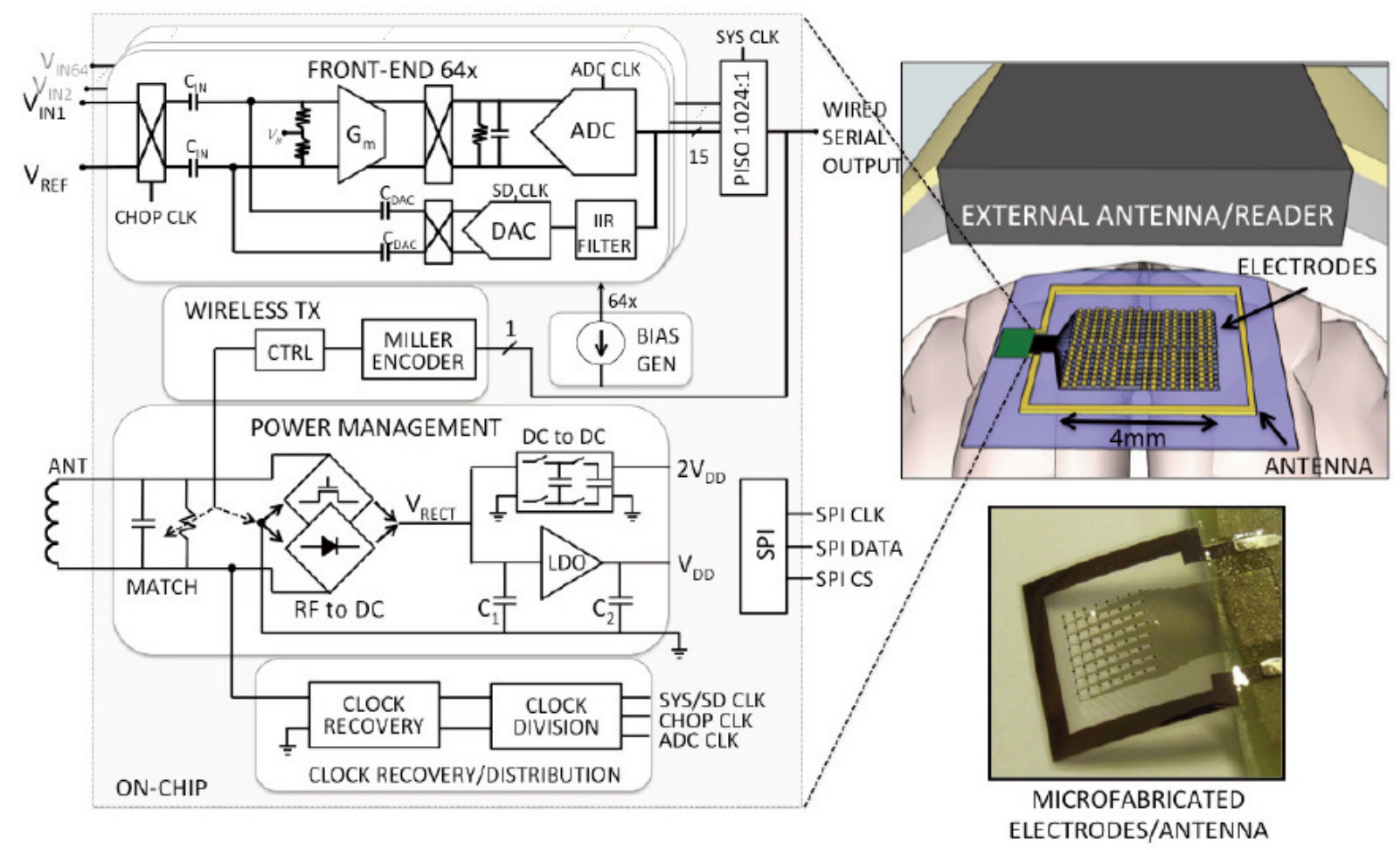

Figura 2.10: Digrama de blocos e ilustração do sistema apresentado em (MULLER et al., 2014). Imagem extraída do artigo (MULLER et al., 2014). 


\subsection{Amplificação de sinais neurais extracelulares}

Os sinais registrados com eletrodos implantados no córtex cerebral contém dois tipos de informação. O primeiro é relacionado à atividade elétrica de neurônios individuais e se evidencia pela presença de spikes os quais revelam a ocorrência de potenciais de ação. Esses spikes possuem uma duração que varia aproximadamente entre $300 \mu \mathrm{s}$ a $1 \mathrm{~ms}$ (GAO et al., 2012). O segundo tipo de informação corresponde à atividade média de vários neurônios relativamente afastados do ponto de registro e que disparam potenciais de ação de maneira mais ou menos síncrona. Esse segundo caso é manifestado através da presença de um onda de baixa frequência $(1 \mathrm{~Hz}$ a $200 \mathrm{~Hz})(\mathrm{GAO}$ et al., 2012).

Na maioria de aplicações de estudo de redes neurais biológicas em animais e de desenvolvimento de neuropróteses motoras, a informação empregada é a relacionada ao disparo de neurônios individuais. Os spike detectados extracelularmente possuem componentes de frequência na faixa de $100 \mathrm{~Hz}$ a $10 \mathrm{kHz}$ e amplitudes que variam na faixa de $50 \mu \mathrm{V}$ a $500 \mu \mathrm{V}$ sendo $100 \mu \mathrm{V}$ o valor típico (MOHSENO; NAJAFI, 2004). Devido às suas pequenas amplitudes, esses sinais precisam ser amplificados no estágio inicial dos sistemas de registro neural. Esse fato foi verificado na seção anterior onde foram apresentados vários exemplos de sistemas implantáveis de registro neural.

Um dos principais problemas relacionados ao projeto de amplificadores de sinais neurais extracelulares é a diferença de potencial DC que se estabelece na interface entre o eletrodo e o eletrolito. Esse potencial eletroquímico depende da concentração iônica ao redor da superfície do eletrodo e por tanto não pode ser conhecida a priori. Essa diferença de potencial pode chegar a valores na ordem de 50mV (WISE; ANGELL, 1975). Esse nível DC deve ser filtrado estabelecendo uma frequência de corte menor que $100 \mathrm{~Hz}$ para o caso de sinais neurais extracelulares, o qual requer uma constante de tempo elevada considerando as limitações de espaço em sistemas implantáveis o portáteis. Diversas estratégias tem sido adotadas ao longo dos anos pelos pesquisadores de amplificadores neurais para a eliminação dessa componente DC conhecida como offset de eletrodo. A necessidade de constantes de tempo elevadas implica na utilização de condensadores ou resistores de valores elevados que se traduz em um consumo elevado de área.

Um dos primeiros trabalhos nesta área propôs o uso de um diodo reversamente polarizado para gerar uma resistência elevada que permitisse a implementação da 
constante de tempo necessária para eliminar o offset de eletrodo sem atenuar o sinal de interesse (NAJAFI; WISE, 1985).

Recentemente foi apontado um problema relacionado à diferença de impedâncias entrada dos amplificadores neurais (NG; XU, 2015). Em sistemas multi-eletrodo, um dos eletrodos é utilizado para fornecer um sinal de referência comum a todos os amplificadores. Como observado na figura 2.11, a impedância vista por este eletrodo é muito menor que a vista pelos outros. Isso traz como consequência uma degradação da capacidade do sistema de rejeitar sinais de modo comum.

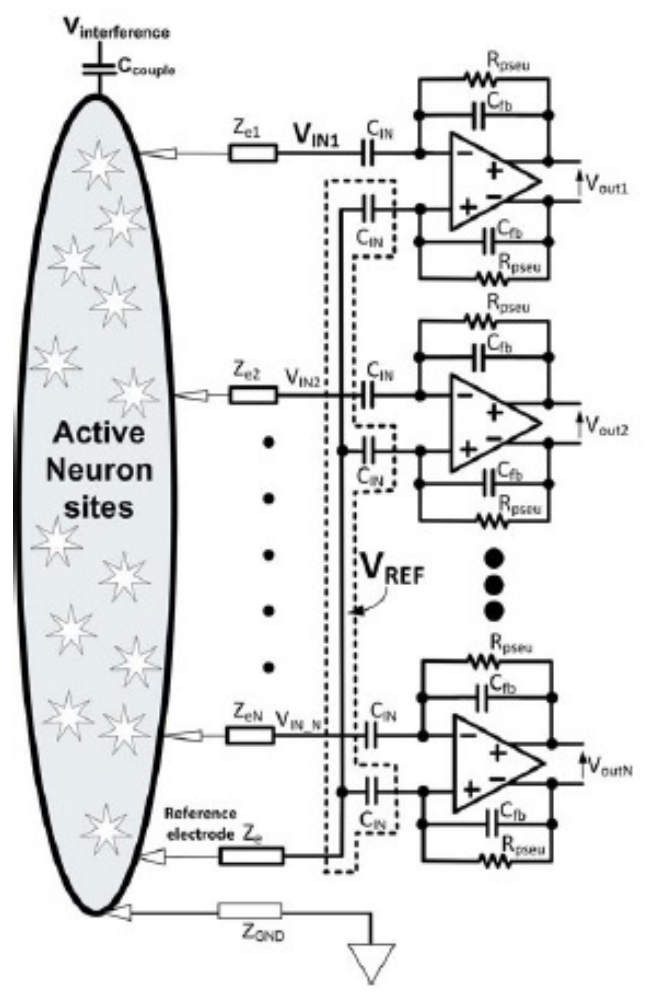

Figura 2.11: Ilustração de um sistema multi-eletrodo de medida de sinais neurais extracelulares. Imagem extraída de (NG; XU, 2015). 


\subsection{Conversores analógico-digital de sinais neurais extracelulares}

O ruído gerado nos eletrodos é considerado para determinar a resolução do ADC de maneira a prevenir um sobre-projeto (over-design) (CHAE; LIU; SIVAPRAKASAM, 2008).

Na tabela 2.1 destacam-se algumas das implementações relatadas recentemente na literatura.

Tabela 2.1: Conversores analógico-digital utilizados em sistemas implantáveis wireless de registro neural

\begin{tabular}{lcccc}
\hline Referência & $\begin{array}{c}\text { Canal de } \\
\text { Publicação }\end{array}$ & $\begin{array}{c}\text { Tensão de } \\
\text { Alimentação } \\
{[\mathrm{V}]}\end{array}$ & $\begin{array}{c}\text { Número } \\
\text { de bits }\end{array}$ & $\begin{array}{c}\text { Consumo } \\
{[\mu \mathrm{W}]}\end{array}$ \\
\hline (AKIN; NAJAFI; BRADLEY, 1998) & JSSC & 5 & 8 & 2000 \\
\hline (ZHENG et al., 2014) & ISCAS & 3.3 & 9 & 54 \\
\hline (TAO; RUSU, 2015) & TCAS & 0.8 & 10 & 9 \\
\hline
\end{tabular}

\subsection{Processamento de sinais neurais extracelulares em circuitos integrados implantáveis}

Entre os diversos tipos de processamentos encontrados em interfaces neurais implantáveis destacam-se:

- Detecção de impulsos elétricos

- Filtragem digital para distinguir entre Local Field Potential e Spikes

- Extração de características

- Separação de impulsos elétricos

Um dos processamentos mais encontrados na literatura é a detecção de impulsos elétricos ou Spike Detection. A detecção de impulsos consiste na indicação da ocorrência de um potencial de ação mediante a análise do sinal coletado por um eletrodo.

Para o caso específico de implantes neurais que transmitem sinais sem fio, a detecção de impulsos permite reduzir a quantidade de dados a serem transmitidos. 
Ao invés de transmitir todas as amostras digitalizadas do sinal registrado por um determinado eletrodo pode-se transmitir apenas os instantes de tempo em que acontecem impulsos elétricos; em algumas aplicações essa informação é suficiente. Por exemplo, mediante a análise da frequência de disparos de neurônios é possível decodificar alguns comandos e controlar neuropróteses ou interfaces cérebro máquina (BMI) (OBEID, 2007). Essa frequência de disparos pode ser calculada conhecendo os instantes de disparos indicado pelo detector de impulsos.

Outro caso é o do uso da detecção de impulsos como indicador do instante em que deve começar um processamento mais complexo. Dessa maneira o processamento mais complexo só é realizado quando acontecer um impulsos elétrico e assim se economiza potência. 


\section{Detecção e separação de impulsos elétricos neurais em circuitos integrados implantáveis - estado da arte}

Como mencionado no capítulo 2 o registro de sinais neurais mediante matrizes de eletrodos implantados no córtex cerebral é um método muito utilizado no âmbito da neurociência experimental e na pesquisa relacionada às neuropróteses. Em ambos casos os sinais registrados são processados para extrair informação relevante para a aplicação. No capítulo 2 foram mencionados vários tipos de processamento implementados dentro das interfaces neurais implantáveis. Podem-se identificar duas motivações para a inclusão desses processamentos nos chips implantáveis. A primeira é viabilizar aplicações de decodificação de sinais neurais e estimulação em tempo real. A outra é diminuir a taxa de transmissão necessária para a leitura de um determinado número de eletrodos.

Neste capítulo estudam-se estratégias para a implementação em hardware da detecção e da separação de impulsos elétricos neurais.

\subsection{Detecção de impulsos elétricos neurais}

O método mais simples para realizar a detecção de impulsos elétricos consiste em comparar a amplitude do sinal medido com um limiar. Devido à sua simplicidade esse método tem sido a escolha mais frequente nos processadores de sinais neurais implantáveis (RIZK; WOLF, 2009). A detecção de spikes pode ser realizada processando diretamente o sinal analógico ou processando as amostras digitalizadas. Um exemplo da detecção analógica é o encontrado no artigo (HARRISON et al., 2006). Nesse artigo descreve-se o uso de um comparador analógico cujo limiar de comparação é deter- 
minado através de um conversor digital-analógico de 7 bits. O LSB (Least Significant Bit) é de $4.8 \mathrm{mV}$ que considerando o ganho 1000 do amplificador que se encontra no estágio prévio ao detector equivale a uma resolução de $4.8 \mu \mathrm{V}$. Esses bits que determinam o limiar de comparação podem ser configurados desde um dispositivo externo através do acoplamento indutivo. O sistema de detecção apresentado em (HARRISON et al., 2006) pode ser representado esquematicamente como na figura 3.1.

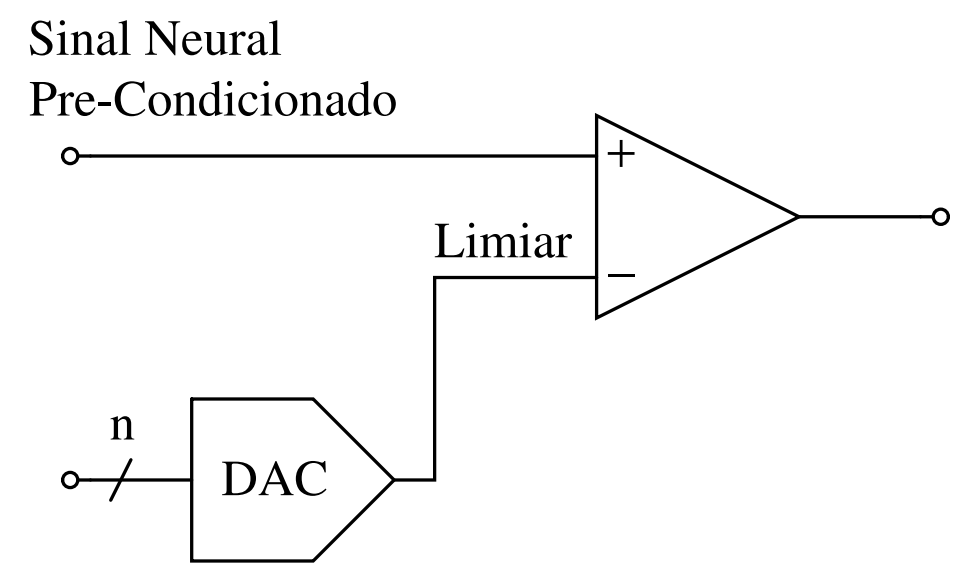

Figura 3.1: llustração do método de detecção de spike por aplicação de limiar simples

Nessa figura o sinal neural precondicionado é o resultado de amplificar e filtrar o sinal registrado por um eletrodo extracelular. O limiar é programado através dos $\mathrm{n}$ bits. Como será comprovado na descrição de outros trabalhos sobre detecção de spike para sistemas implantáveis, a programabilidade do limiar é uma característica comum. Embora existam tentativas de gerar o limiar automaticamente a partir da análise de sinal neural a alternativa programável continua sendo mais robusta. Para a definição do limiar programável o sinal neural deve ser transmitido a um computador externo onde é analisado sem limitações de recursos de computacionais. Como resultado dessa análise o limiar ótimo é obtido. O operador, então, programa esse limiar através dos recursos de comunicação disponíveis no sistema implantável.

Outro exemplo da comparação com um limiar programável é encontrado no artigo (SODAGAR; WISE; NAJAFI, 2006) apresentado na IEEE Biomedical Circuits and Systems Conference de 2006. Nesse trabalho apresenta-se um processador de sinais neurais implantável, que possui a capacidade de detecção de spikes mediante a aplicação de limiar. Esse limiar pode ser aplicado às amostras positivas, às negativas ou a ambas. Na figura 3.2, extraída de (SODAGAR; WISE; NAJAFI, 2006) apresenta-se uma ilustração dessas três possibilidades de aplicação de limiar. 


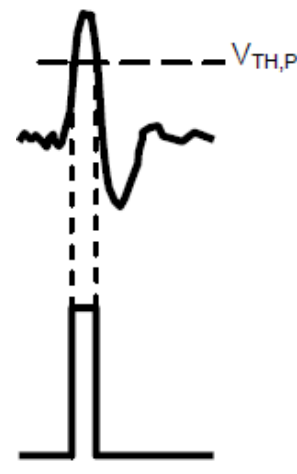

(a)

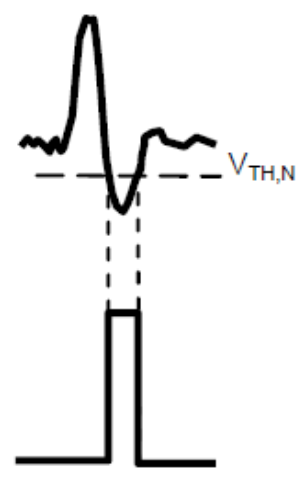

(b)

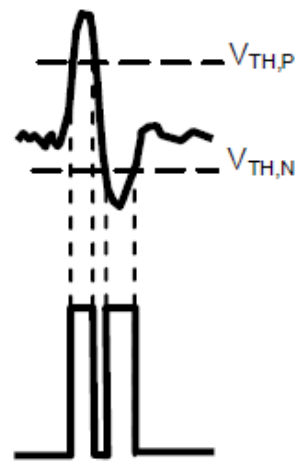

(c)

Figura 3.2: Modos de detecção suportados pelo processador apresentado em (SODAGAR; WISE; NAJAFI, 2006). (a) Positivo (b) Negativo (c) Bifásico. Imagem extraída de (SODAGAR; WISE; NAJAFI, 2006).

Também existem implementações onde o limiar é calculado automaticamente a partir da análise do sinal dentro do circuito integrado implantável. Um exemplo é o relatado por Reid Harrison em (HARRISON, 2003). A ideia da proposta de Harrison é gerar automaticamente um limiar que seja maior ao ruído de fundo. Na figura 3.3, extraída desse artigo, observa-se o esquema da proposta.

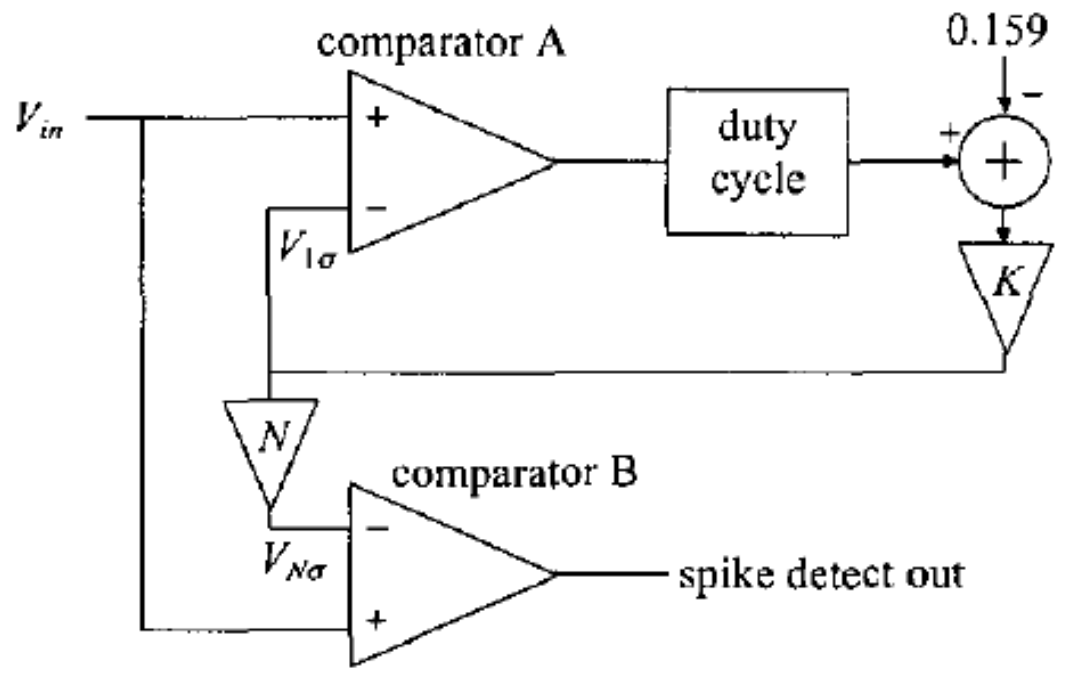

Figura 3.3: Estratégia para geração de limiar de comparação apresentada em (HARRISON, 2003). Imagem extraída de (HARRISON, 2003).

O objetivo desse circuito é estimar o desvio padrão do ruído de fundo e estabelecer com limiar de comparação um múltiplo $(5 \times)$ desse valor. A premissa adotada por Harrison foi que o sinal de entrada do detector é uma combinação linear de impulsos elétricos neurais de duração aproximada de $1 \mathrm{~ms}$ e ruído gaussiano limitado em banda 
de frequência com média 0 . Assume-se então que o sinal registrado pelo eletrodo é aplicado a um filtro passa banda, como costuma acontecer, e a saída desse filtro é aplicada ao detector.

\subsubsection{Aplicação de pré-ênfase ao sinal}

Para lidar com o problema do alto conteúdo de ruído, costuma-se adicionar um estágio de pré-ênfase ao processo de detecção de impulsos elétricos (GIBSON; JUDY; MARKOVIĆ, 2008), (MUKHOPADHYAY; RAY, 1998). Esse pré-processamento tem como objetivo enfatizar os spikes neurais e atenuar o ruído de fundo.

Um exemplo da aplicação de um pré-processamento para pré-ênfase do sinal é o encontrado no trabalho da equipe do professor Wouter Serdijn da Universidade Técnica de Delft (HISENI et al., 2009).

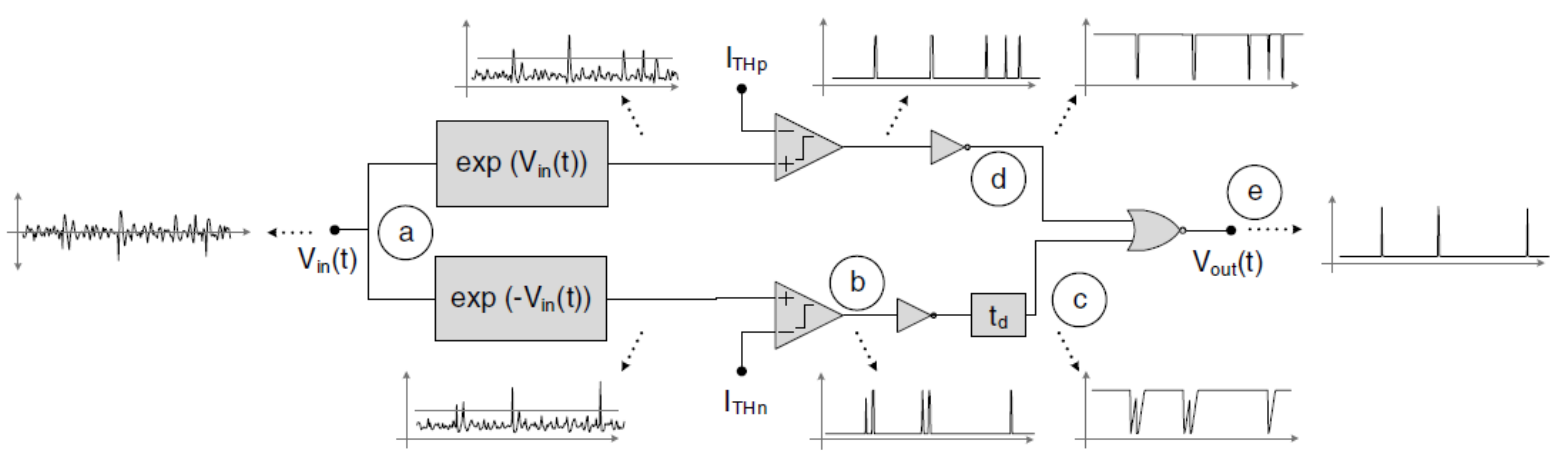

Blockdiagram of the proposed dual threshold AP detector.

Figura 3.4: Implementação analógica de detecção de spikes com estágio de pré-ênfase. Imagem extraída de (HISENI et al., 2009).

A estratégia adotada pela equipe do professor Serdijn foi a aplicação da função exponencial ao sinal de entrada, sob o argumento que este operador distancia os sinais de maior amplitude (spikes) dos de menor amplitude (ruído). Após esse afastamento de spikes e ruído fica mais fácil separá-los mediante um comparador. O operador exponencial é um circuito analógico que recebe como entrada a tensão e gera uma corrente proporcional ao exponencial da tensão de entrada. Essa corrente é comparada com um limiar aplicado externamente. O operador é aplicado tanto ao sinal quanto ao negativo do sinal para poder detectar picos positivos e negativos. Nesse aspecto o sistema adota um esquema de limiar bifásico, pois só aceita um spike quando tanto a parte positiva quanto a negativa são suficientemente altas. Tanto o limar positivo $\left(I_{T H P}\right)$ quanto o negativo $\left(I_{T H N}\right)$ são aplicados externamente nesse sistema. 
A estratégia de pré-ênfase mais encontrada na literatura, considerando implementações em hardware, é a aplicação do operador não linear de energia conhecido como NEO (não linear energy operator) ou TEO (Teager energy operator).

\subsubsection{Operador não linear de energia}

O operador não linear de energia (NEO) conhecido também como operador Teager tem duas versões, uma em tempo continuo definida como:

$$
\psi[x(t)]=\left[x^{\prime}(t)\right]^{2}-x(t) x^{\prime \prime}(t)
$$

e outra em tempo discreto definida como:

$$
\psi[x(n)]=[x(n)]^{2}-x(n-1) x(n+1)
$$

Ambas versões foram desenvolvidas por Teager no seu trabalho sobre modelagem não linear de voz (TEAGER; TEAGER, 1989) e estudado sistematicamente por Kaiser em (KAISER, 1990a) e (KAISER, 1990b). A aplicação desse operador como processamento de pré-ênfase para detecção de spikes foi proposta por Mukhopadhyay em (MUKHOPADHYAY; RAY, 1998). Embora Mukhopadhyay tenha apresentado uma análise aprofundada que prova a utilidade do operador NEO na pré-ênfase de spikes outros autores tem dado argumentos mais intuitivos. Por exemplo, Sarah Gibson em (GIBSON; JUDY; MARKOVIĆ, 2008) afirma que o resultado da versão discreta do operador NEO é um valor alto quando tanto a amplitude quanto a frequência do sinal são altos (condição necessária para a ocorrência de um spike) pois a amostra n seria grande e ademais maior que as duas amostras adjacentes $(n+1$ e $n-1)$. Ou seja, escolhendo um limiar suficientemente elevado na comparação da saída do operador NEO, garantimos que apenas a ocorrência do mencionado evento seja reconhecida como spike, pois estes são caracterizados por um aumento simultâneo da amplitude e da frequência.

Observar apenas amplitude não é suficiente para detectar um spike pois em muitos casos o ruído de fundo pode ter amplitudes similares às dos spikes. Esse fato é ilustrado na figura 3.5 .

As duas curvas dessa figura tem valor similar de $x(n)$ porém só a primeira tem características de variação rápida. O operador NEO fornecerá um valor muito maior para o caso da primeira curva. 

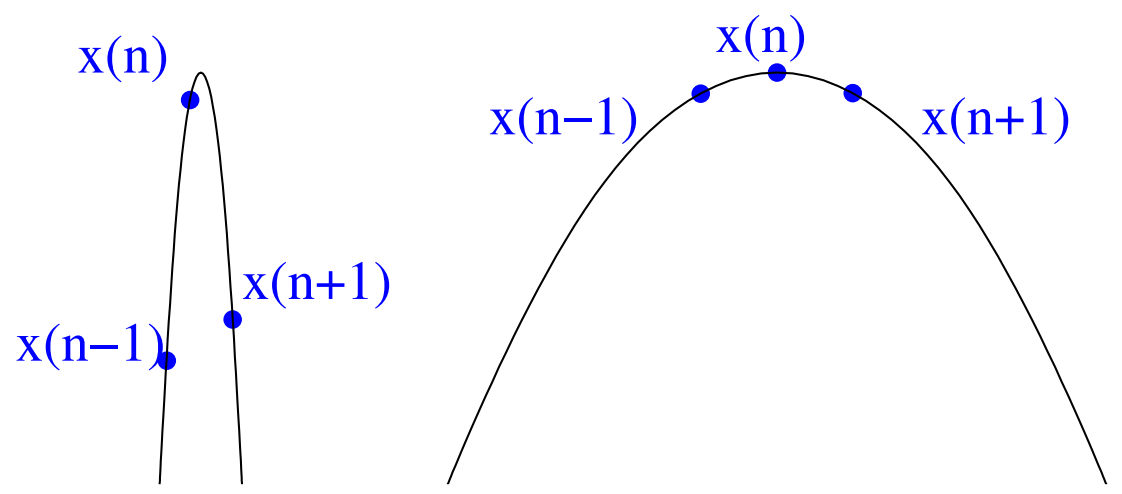

Figura 3.5: llustração da ideia que a amplitude não é parâmetro suficiente para caracterizar um spike

A frequência também não é um parâmetro suficiente para detectar os spikes porque o ruido de fundo composto pela atividade de muitos neurônios afastados do eletrodo tem o mesmo conteúdo de frequência. Então um filtro linear de frequência não seria suficiente para remover o ruído de fundo. É importante lembrar que a ideias que acabamos de comentar são argumentos intuitivos da utilidade do operador NEO. Uma prova formal da importância do NEO pode se encontrar no trabalho de Mukhopadhyay em (MUKHOPADHYAY; RAY, 1998).

Devido à sua simplicidade e eficácia na detecção em sinais ruidosos o operador NEO tem sido a escolha preferida dos pesquisadores para implementação de sistemas detecção de spikes para interfaces neurais implantáveis. A seguir apresentam-se alguns dos trabalhos nessa linha.

Em (HOLLEMAN et al., 2008) apresenta-se a implementação analógica do operador NEO em tempo contínuo. Na figura 3.6 observa-se o diagrama esquemático dessa implementação. De acordo com esse diagrama de blocos a saída tem a seguinte expressão:

$$
N E O=\left(\frac{\mathrm{d} V_{i n}(t)}{\mathrm{d} t}\right)^{2}-\left(\frac{\mathrm{d}^{2} V_{i n}(t)}{\mathrm{d} t^{2}}\right) \times V_{i n}(t)
$$

Porém, a implementação é em modo corrente e a saída é diferencial. A saída de corrente diferencial é proporcional à expressão dada em 3.3. 


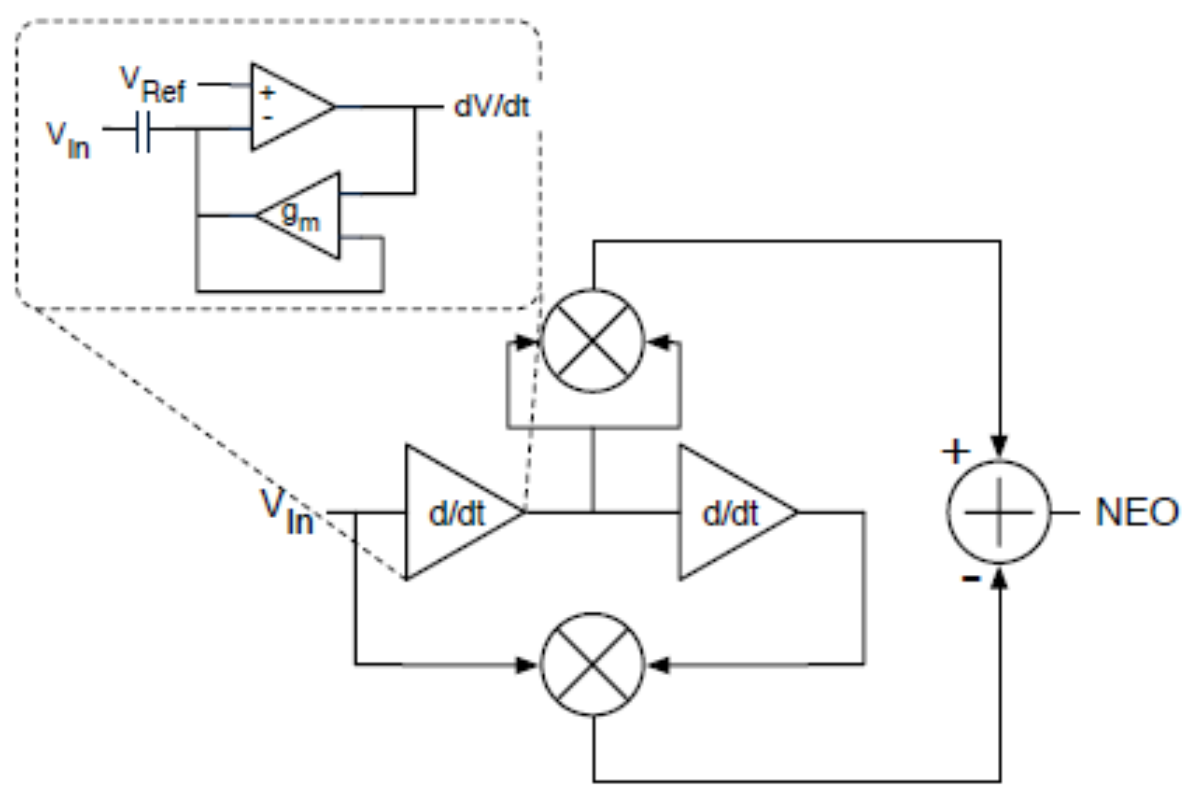

Figura 3.6: Implementação analógica do NEO apresentada em (HOLLEMAN et al., 2008). Imagem extraída de (HOLLEMAN et al., 2008).

Outra implementação analógica do NEO em tempo continuo foi publicada no periódico Electronics Letters da IET (LI; XU, 2011). Na figura 3.7 observam-se o diagrama de blocos, similar ao do trabalho anterior, e o multiplicador baseado no princípio translinear.

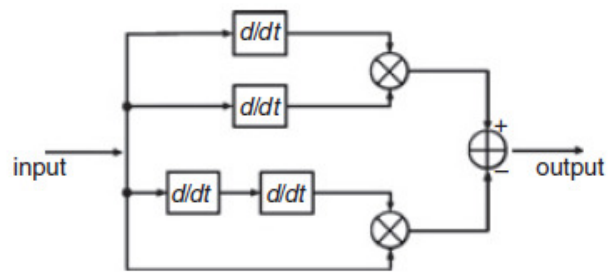

Diagram of $\mathrm{NEO}$ detector

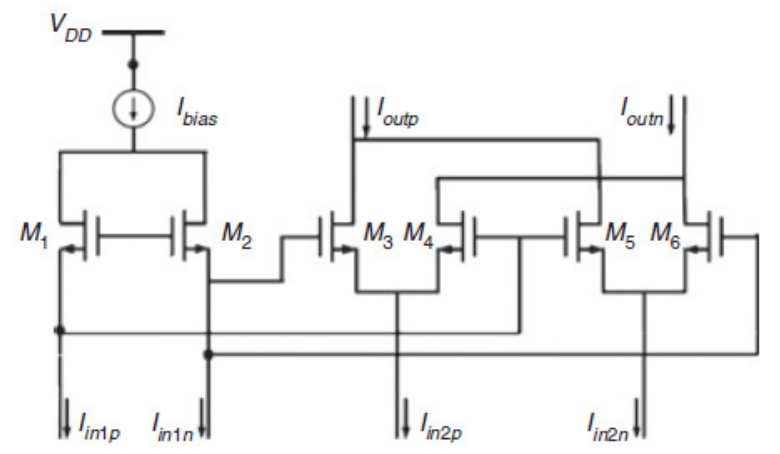

Schematic of multiplier

Figura 3.7: Implementação analógica do NEO apresentada em (LI; XU, 2011). Imagem extraída de (LI; XU, 2011). 
Em (GOSSELIN; SAWAN, 2009) os professores Gosselin e Mohamad Sawan da Escola Politécnica de Montreal apresentaram uma implementação analógica do NEO em tempo continuo. O diagrama de blocos dessa implementação é mostrado na figura 3.8. O bloco differentiator contém dois derivadores implementados com transcondutores.

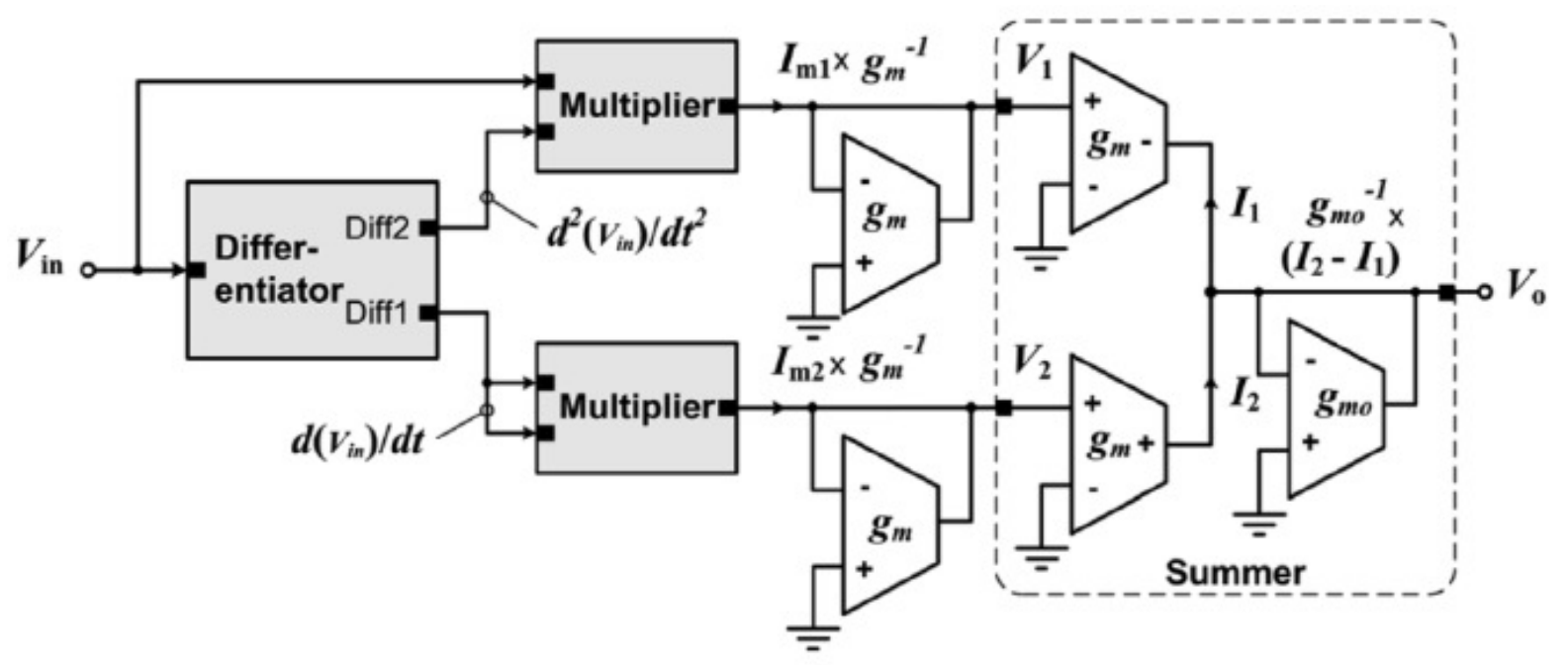

Figura 3.8: Implementação analógica do NEO. Imagem extraída do artigo (GOSSELIN; SAWAN, 2009). 


\subsection{Separação de impulsos elétricos neurais}

Como apontado nas seções anteriores, o registro de sinais neurais através de microeletrodos implantáveis é uma ferramenta importante para a neurociência. Também, verificou-se o grande potencial dessa ferramenta em tecnologias médicas para os tratamentos de paralisia, epilepsia e perda de memória. Nesta seção, descreve-se um tipo de processamento dos sinais registrados por cada eletrodo, o qual se denomina "Separação de Impulsos" ou "Spike Sorting".

Considerando-se cada eletrodo de forma separada, este recebe sinais elétricos de vários neurônios simultaneamente. Quando acontece um potencial de ação em um neurônio, o eletrodo situado no meio extracelular próximo a este neurônio registra um sinal elétrico conhecido como impulso elétrico ou spike. O processo de identificação de todos os impulsos elétricos que representam a atividade de um mesmo neurônio é denominado como "Separação de Impulsos" ou "Spike Sorting".

É importante salientar que o potencial de ação gerado em um neurônio é diferente do impulso elétrico (spike) registrado pelo eletrodo. O primeiro só pode ser medido intracelularmente, enquanto que o segundo, apenas extracelularmente. Porém, é possível obter o padrão temporal de disparos de potenciais de ação em um neurônio mediante o registro de spikes.

No artigo (RUTISHAUSER; SCHUMAN; MAMELAK, 2006) aponta-se que para um melhor entendimento das funções neurais é necessário conhecer de maneira individual as atividades elétricas de vários neurônios e portanto é muito importante atribuir com exatidão cada spike individual a um neurônio particular.

O spike sorting tem sido realizado majoritariamente em um computador externo mediante a análise do sinal registrado durante algum tempo. Ou seja, primeiro se coleta o sinal mediante a matriz multi-eletrodo, o sinal se transmite a um computador externo e neste dispositivo se executam complexos algoritmos, a maioria deles baseado em PCA (Principal Component Analysis), para separar os spikes. Essa metodologia é conhecida como Offline Sorting.

Na busca de sistemas implantáveis que operem em tempo real, recentemente tem aumentado a procura por soluções mais automáticas. A ideia consiste em separar cada impulso imediatamente depois que este acontece. Essa técnica é conhecida como Online Sorting. 
Na figura 3.9, ilustra-se o processo de Spike Sorting. O sinal neural registrado extracelularmente por um eletrodo contém impulsos elétricos que correspondem à atividade elétrica de vários neurônios. Os impulsos elétricos são detectados comumente através de um processo de limiarização. No exemplo apresentado nessa figura, os impulsos elétricos detectados correspondem à atividade elétrica de dois neurônios. Como se observa, o impulso elétrico provocado por um neurônio tem um formato diferente daquele provocado por outro. Por meio do processo de separação de impulsos, é possível identificar aqueles que correspondem a um ou a outro neurônio.
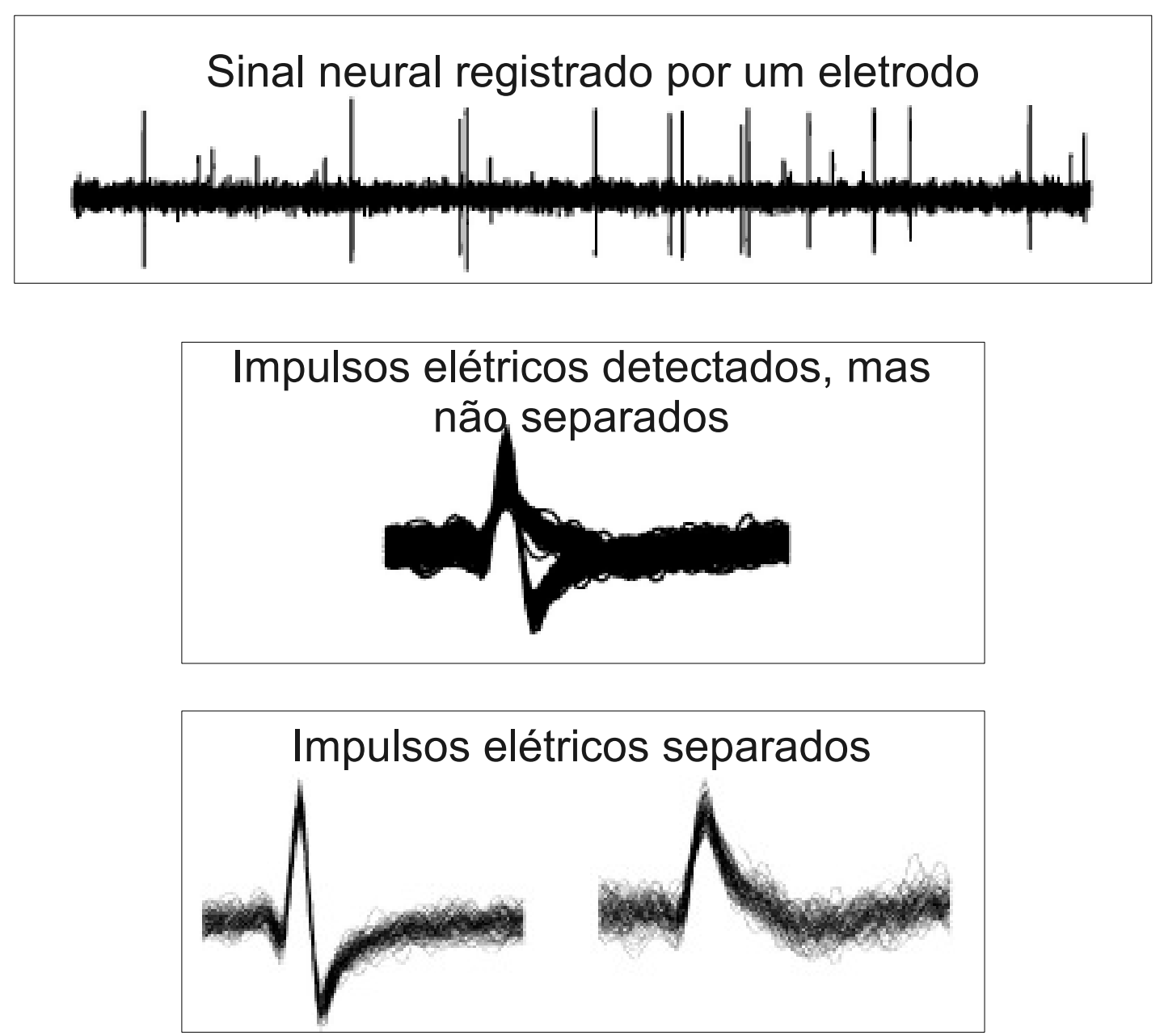

Figura 3.9: Ilustração do processo de Spike Sorting. A partir do sinal registrado por um único eletrodo (figura superior) os impulsos elétricos são detectados mediante um processo de limiarização e, finalmente, separados. Figura adaptada de (QUIROGA; NADASDY; BEN-SHAUL, 2004)

O formato dos impulsos elétricos permite realizar o processo de separação, porém sabe-se que para um mesmo neurônio o formato do sinal que este gera no eletrodo não contém informação nenhuma. Portanto após a fase de identificação dos impulsos elétricos, a única informação que é passada para o seguinte estágio de processa- 
mento é o conjunto dos instantes de disparos correspondentes a cada neurônio. Com isso se consegue uma redução importante da quantidade de dados a ser transmitida desde uma interface neural implantável.

Na figura 3.10 apresenta-se outra ilustração do processo de Spike Sorting.

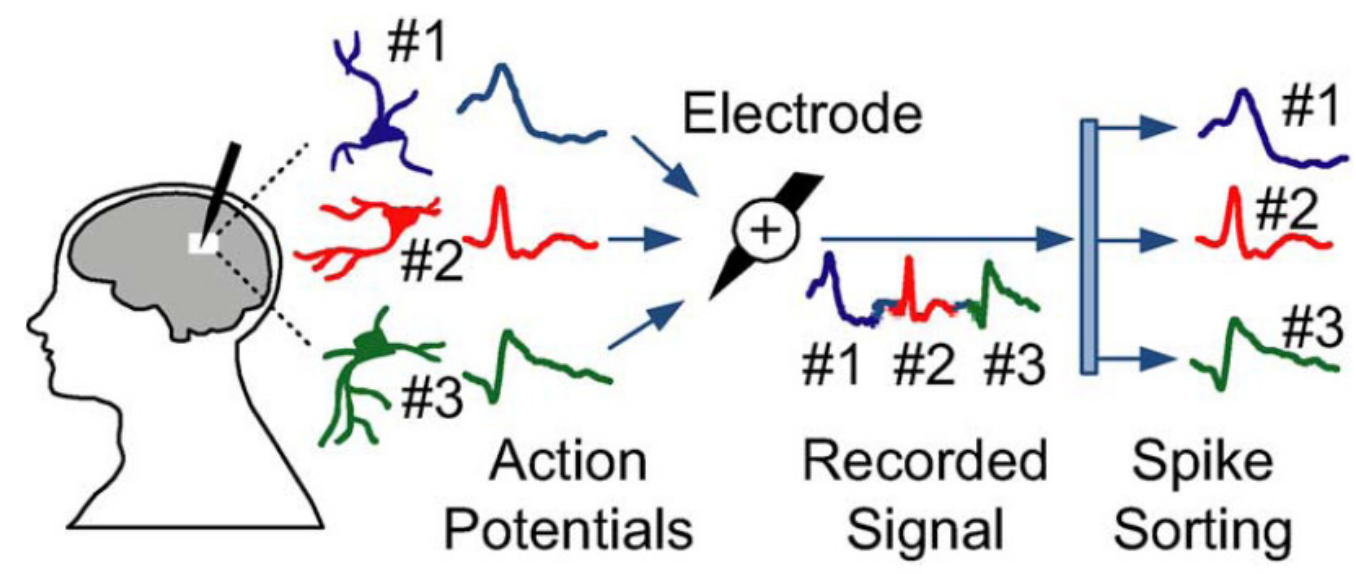

Figura 3.10: llustração do processo deSpike Sorting criada por Karkare e Gibson (KARKARE; GIBSON; MARKOVIC, 2013). Imagem extraída de (KARKARE; GIBSON; MARKOVIC, 2013).

Como apontado em (GIBSON; JUDY; MARKOVIĆ, 2008), o processo de separação de impulsos elétricos é importante por dois motivos. O primeiro, funcional, está relacionado ao fato de que mesmo que dois neurônios estejam muito próximos, eles podem codificar informações completamente diferentes. Os neurocientistas frequentemente precisam identificar quais spikes correspondem a quais neurônios com o intuito de entender a circuitaria neural. As interfaces cérebro máquina, por sua vez, dependem geralmente da atividade elétrica de neurônios individuais. O segundo motivo, de caráter prático, refere-se à redução da quantidade de dados a ser enviados ao exterior do chip implantado. Limitações de largura de banda para comunicação e de consumo de potência requerem uma redução de dados dentro do chip. Para tal efeito, recorre-se ao Spike Sorting. 


\subsection{Proposta para utilização dos sistemas de detecção e classificação de spikes}

Os sistemas de detecção e classificação desenvolvidos nesta tese podem ser inseridos num sistema de processamento de sinais neurais que pode ser representado como na figura 3.11, na qual os blocos ressaltados com cor celeste são desenvolvidos nesta tese. Nessa figura, $\mathrm{S}$ representa o sinal neural pré-condicionado, $\mathrm{T}_{\text {spk }}$ representa a informação sobre o instante em que ocorre cada spike detectado e $\mathrm{C}_{\mathrm{spk}}$ representa a classe à qual corresponde o spike detectado. Nessa figura, alguns sinais de controle foram omitidos.

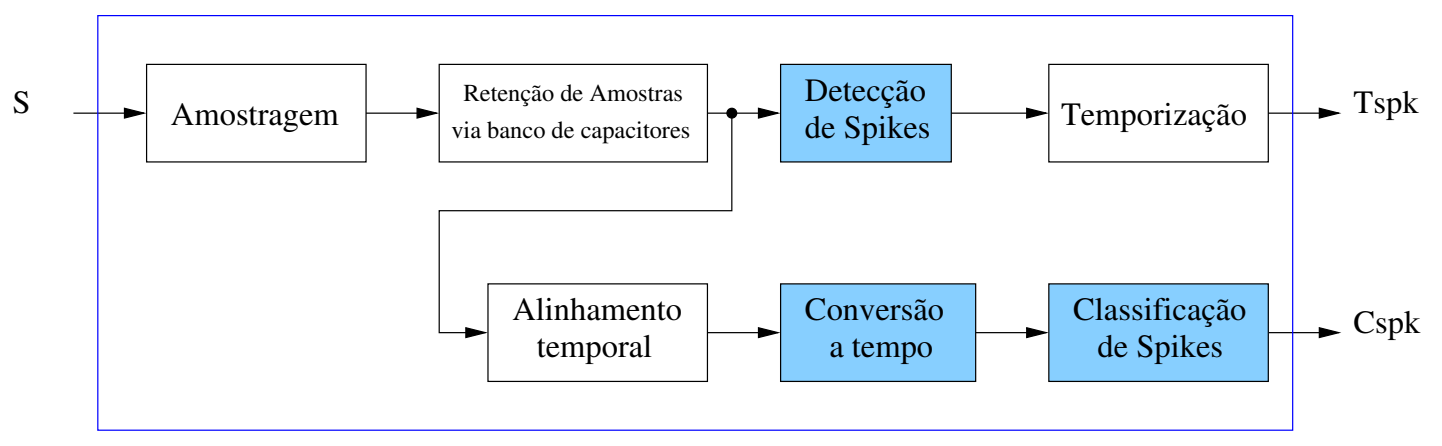

Figura 3.11: Proposta para utilização dos sistemas desenvolvidos nesta tese dentro de um sistema de processamento de sinais neurais

O sistema de detecção desenvolvido nesta tese e detalhado no capítulo 4, baseado no operador NEO, precisa de três amostras para determinar a ocorrência de um spike (conforme a figura 3.5). Uma abordagem para a retenção dessas amostras consiste no uso de um banco de capacitores. O banco de capacitores também pode armazenar as amostras necessárias para realizar o processo de classificação de spikes. Para viabilizar diversas aplicações, inclusive $\mathrm{BCl}$, é importante dispor de um estágio de temporização. Esse estágio permite fornecer informação relacionada ao instante de ocorrência dos spikes, como por exemplo o tempo entre spikes consecutivos.

O sistema de classificação desenvolvido nesta tese e apresentado no capítulo 5 é baseado na representação de amostras através atrasos entre pulsos digitais (representação baseada em tempo). Para obter essa representação é necessário utilizar um conversor de tensão a tempo (o qual também é detalhado no capítulo 5). A classificação opera com um conjunto de amostras das quais pretende-se extrair informação relacionada ao formato do spike. O sistema de classificação desenvolvido nesta tese baseia-se na comparação de formatos de spikes. Portanto, é importante 
que os conjuntos de amostras que representam esses formatos estejam alinhados temporalmente, tomando como referência alguma característica desse formato. $\mathrm{Na}$ revisão da literatura encontraram-se vários casos em que a amplitude máxima do sinal é utilizada como a característica de referência para o alinhamento temporal (PARASKEVOPOULOU; BARSAKCIOGLU; SABERI, 2013), (ZAMANI; DEMOSTHENOUS, 2014), (NAVAJAS et al., 2014); porém, outras características também são possíveis (ZVIAGINTSEV; PERELMAN; GINOSAR, 2006), (CHOI; JUNG; KIM, 2006).

É necessário lembrar que os sistemas que não são abordados nesta tese, como por exemplo o de alinhamento temporal, são necessários para garantir uma funcionalidade completa do sistema de processamento de sinais neurais. Esses outros sistemas devem ser considerados como propostas de trabalhos futuros. 


\section{Desenvolvimento de um sistema de detecção de impulsos elétricos neurais para uma interface neural implantável}

Este capítulo descreve o desenvolvimento de um sistema de detecção de impulsos elétricos neurais apropriado para a sua integração mediante tecnologia CMOS como parte de uma interface neural implantável. Como estudado no capítulo 3, este tipo de sistemas é composto por um estágio de pré-ênfase do sinal seguido de um estágio de comparação com um limiar. Também foi mencionado que, devido à sua simplicidade, a estratégia mais empregada para o pré-ênfase em interfaces neurais implantáveis é a aplicação do operador não linear de energia Teager (NEO).

O sistema apresentado neste capítulo processa diretamente amostras analógicas do sinal de entrada. Essas amostras de tensão são convertidas a correntes elétricas que são aplicadas a um circuito que realiza a operação NEO.

$\mathrm{Na}$ figura 4.1 apresenta-se o diagrama de blocos do sistema desenvolvido. Nessa figura, $V_{\text {in }}$ representa a tensão que é resultado da amplificação e filtragem do sinal medido por um eletrodo.

O sinal de entrada $\left(V_{i n}\right)$ é amostrado sucessivamente em três capacitores. O bloco VTC (Voltage to current converter), converte as amostras analógicas de tensão em correntes, as quais são aplicadas ao circuito que realiza a operação NEO. O resultado dessa operação é a corrente $I_{\text {neo }}$ que é comparada com uma corrente de referência que pode ser escolhida através do conversor digital-analógico.

Observam-se também chaves com três posições cuja necessidade será explicada a seguir. Na aplicação do operador NEO a ordem de ocorrência das amostras é importante: o produto da amostra seguinte com a amostra anterior deve ser subtraído 


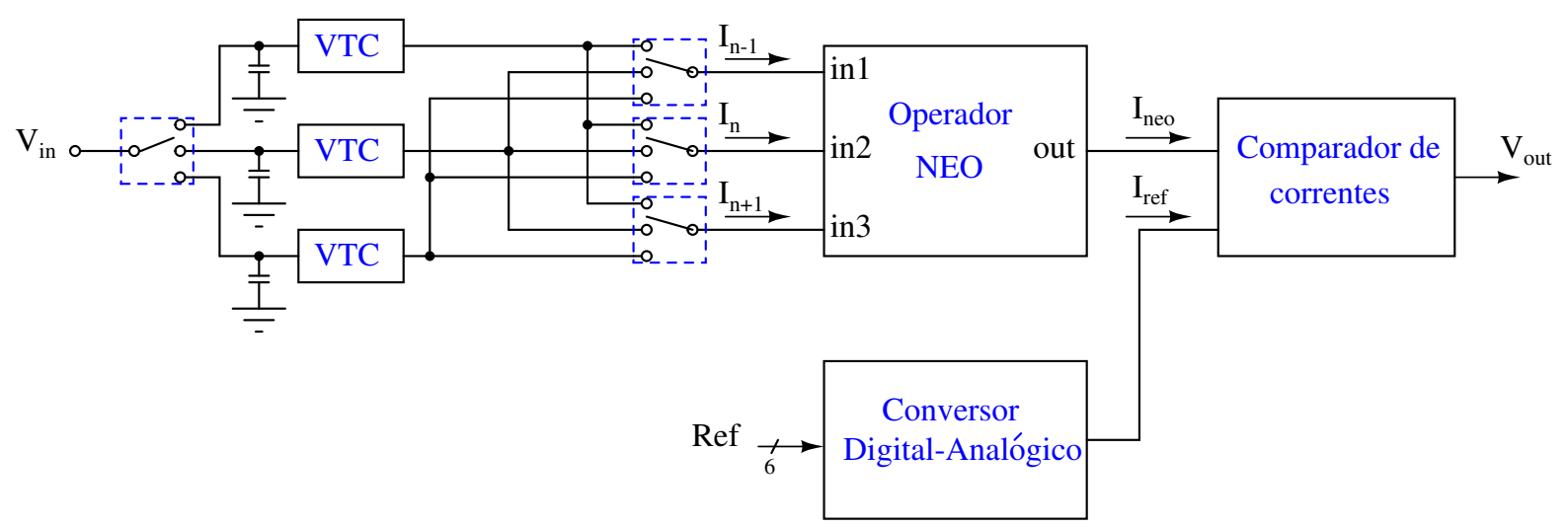

Figura 4.1: Diagrama de blocos do sistema de deteç̧ão baseado no operador NEO. O bloco denominado VTC é um conversor de tensão a corrente (Voltage to current converter)

do quadrado da amostra presente. As chaves que ligam as saídas dos circuitos VTC às entradas do circuito NEO devem ser controladas de maneira que essa ordem de aplicação das amostras seja garantida. Na figura 4.1, não se inclui o circuito que realiza esse controle das chaves analógicas, porém será explicado numa das seções deste capítulo.

\subsection{Análise da aplicação do operador Teager ao valor absoluto do sinal neural extracelular}

De acordo com a interpretação de (GIBSON; JUDY; MARKOVIĆ, 2008), o resultado do operador NEO é um valor alto só nos instantes em que o sinal apresenta um conteúdo alto de potência e ao mesmo tempo de frequência. O conteúdo de potência é alto quando o termo $x^{2}(n)$ é elevado e o de frequência é alto quando $x(n)$ é alto e ao mesmo tempo $x(n-1)$ e $x(n+1)$ tem valores pequenos. Sem importar se os valores das amostras são positivas ou negativas, o operador NEO fornece um resultado elevado na presença de um spike, o qual é coerente com o fato que os spikes podem ser positivos ou negativos.

Em relação à simplicidade em termos de hardware da implementação do operador NEO que será apresentada nas seções seguintes, é mais conveniente trabalhar apenas com os valores absolutos das amostras. Considerando a interpretação de (GIBSON; JUDY; MARKOVIĆ, 2008) mencionada no parágrafo anterior, a escolha de trabalhar apenas com os valores absolutos das amostras é válida. 
Para uma avaliação mais objetiva da influência da escolha adotada no resultado da detecção de spike realizaram-se simulações em Matlab. Mediante essas simulações compara-se a opção de trabalhar com os valores absolutos das amostrar e a opção original de considerar o sinal das amostras. Em ambos os casos o sinal é aplicado a um operador NEO implementado em Matlab e o sinais resultantes são comparados tanto visualmente quanto através de parâmetros de detecção. A seguir apresentamse comparações visuais de ambos métodos. Nas figuras 4.2 e 4.3 apresentam-se os resultados da aplicação do NEO aos sinais e aos valores absolutos dos sinais do banco de dados Waveclus. Observam-se $300 \mathrm{~ms}$ de cada sinal. Pode-se comprovar que a aplicação do NEO ao valor absoluto do sinal fornece os mesmos resultados, em termos de enfatizar a presença de spikes, que a aplicação do NEO ao sinal original.
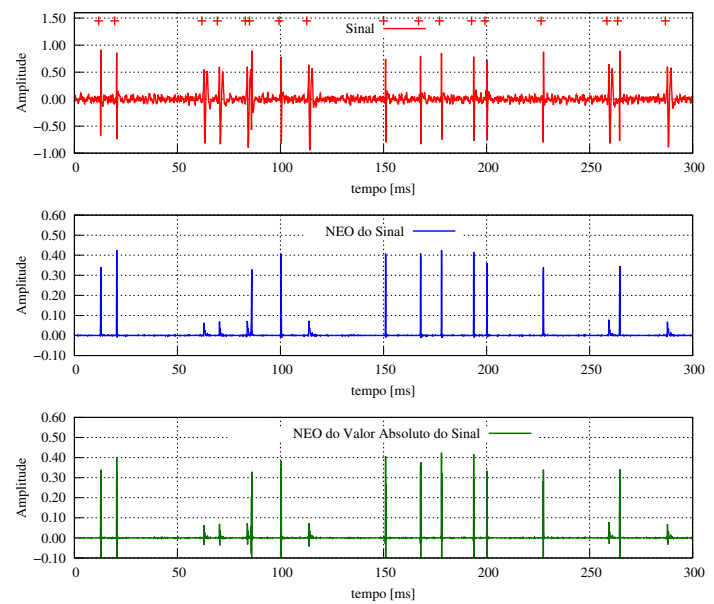

(a) Sinal com $5 \%$ de ruído
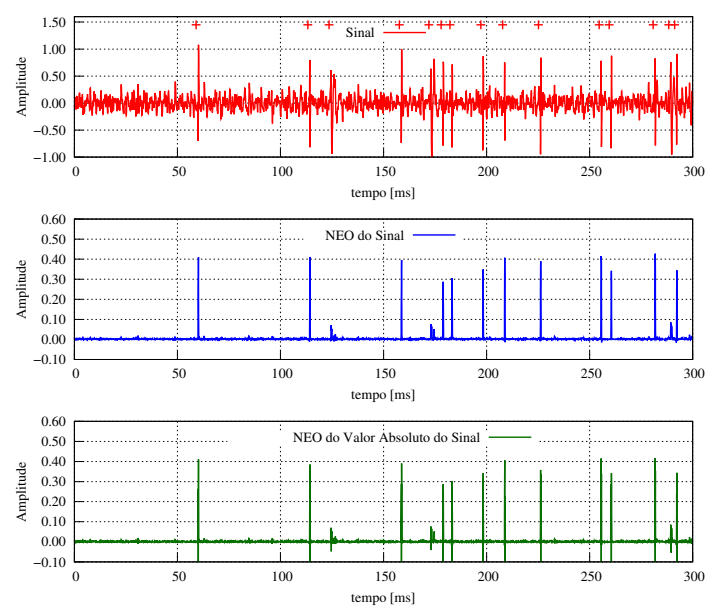

(c) Sinal com $15 \%$ de ruído
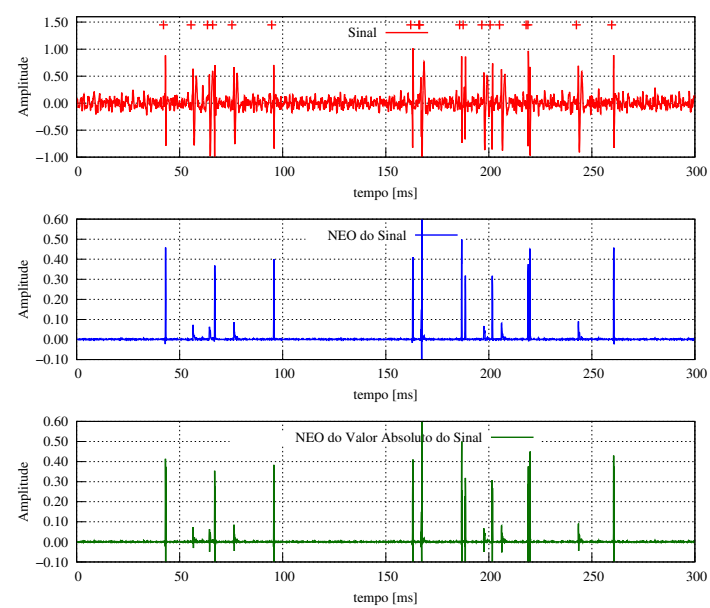

(b) Sinal com $10 \%$ de ruído
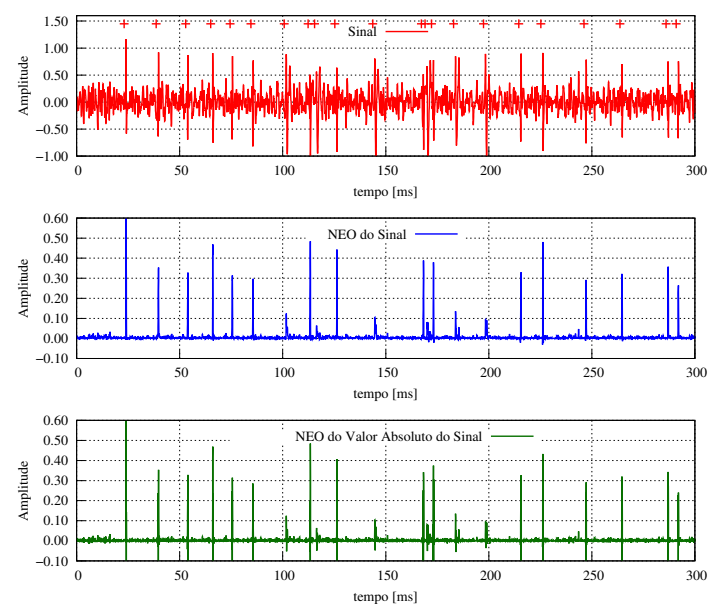

(d) Sinal com $20 \%$ de ruído

Figura 4.2: Resultado da aplicação do NEO implementado em Matlab aos sinais e aos valores absolutos dos sinais do banco de dados Waveclus. Sinais com ruídos de $5 \%, 10 \%, 15 \%$ e $20 \%$ 

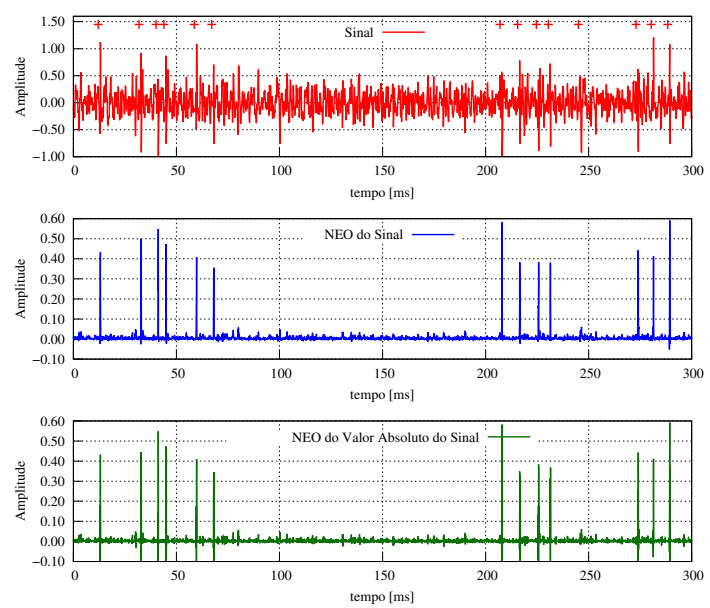

(a) Sinal com $25 \%$ de ruído
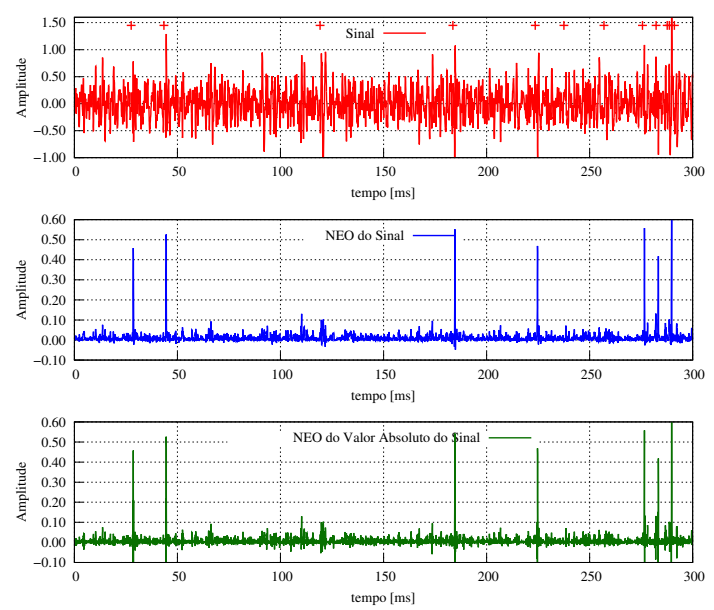

(c) Sinal com $35 \%$ de ruído
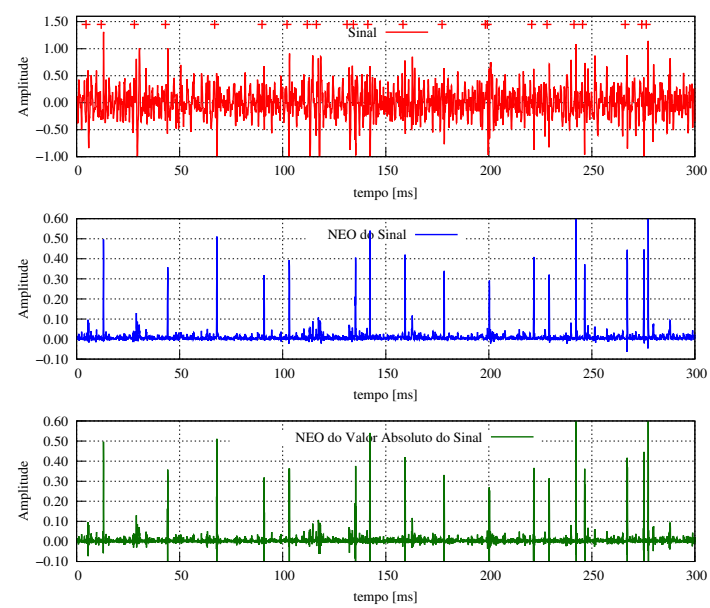

(b) Sinal com $30 \%$ de ruído
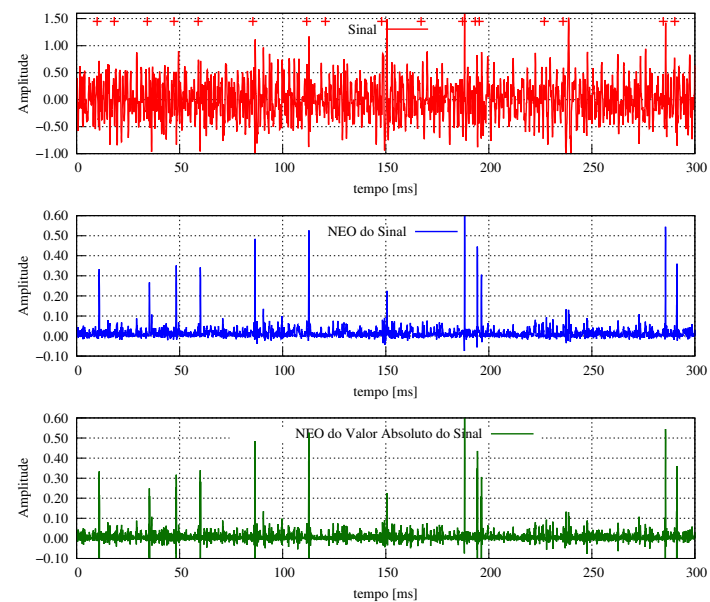

(d) Sinal com $40 \%$ de ruído

Figura 4.3: Resultado da aplicação do NEO implementado em Matlab aos sinais e aos valores absolutos dos sinais do banco de dados Waveclus. Sinais com ruídos de $25 \%, 30 \%, 35 \%$ e $40 \%$.

Embora a comparação visual evidencia a validade da hipótese sobre a equivalência entre usar o sinal ou o valor absoluto do sinal no estágio de pré-ênfase, é importante realizar medidas mais objetivas. Para tal propósito comparam-se parâmetros de detecção tais como a taxa de verdadeiros positivos e a taxa de falsos positivos. $\mathrm{O}$ algoritmo de detecção implementado em Matlab consiste em comparar as amostras da saída do estágio de pré-ênfase com um determinado limiar, e quando alguma ultrapassar o limiar registra-se a ocorrência de um spike. Considera como spike a uma janela que começa $1 \mathrm{~ms}$ antes do instante de ultrapassagem do limiar e termina $2 \mathrm{~ms}$ depois desse instante. Essa restrição a $3 \mathrm{~ms}$ é baseada no fato que é pouco provável que aconteçam spikes de maior duração e na necessidade de minimizar a probabilidade de agrupar 2 spikes numa mesma janela (GIBSON; JUDY; MARKOVIĆ, 2008), (GIBSON, 2010). 
Os parâmetros de detecção mencionados anteriormente dependem do limiar escolhido. Para o caso de detecção sem pré-ênfase escolheu-se como limiar:

$$
T h r=4 \cdot \sigma_{N}
$$

onde $\sigma_{N}$ é uma estimativa do desvio padrão do ruído, dada por:

$$
\sigma_{N}=\operatorname{mediana}\left\{\frac{|x(n)|}{0.6745}\right\}
$$

Tais escolhas de $T h r$ e $\sigma_{N}$ foram adotadas dos trabalhos de (QUIROGA; NADASDY; BEN-SHAUL, 2004) e Donoho1994.

Para o caso de detecção usando NEO como pré-ênfase escolheu-se o seguinte limiar:

$$
T h r_{N E O}=C \frac{1}{N} \sum_{n=1}^{N} \psi[x(n)]
$$

que representa um múltiplo da média do NEO do sinal. $\mathrm{N}$ é o número de amostras e C é um fator que para este caso foi 8 . Estas escolhas correspondem ao estudo de (GIBSON, 2010).

Na tabela 4.1 apresentam-se os parâmetros obtidos após a aplicação do algoritmo de detecção sobre os sinais originais do banco de dados Waveclus, sobre o NEO do

\begin{tabular}{|c|c|c|c|c|c|c|}
\hline $\begin{array}{c}\text { Ruído } \\
\text { no Sinal [\%] }\end{array}$ & \multicolumn{2}{|c|}{$\begin{array}{l}\text { Sinal Original } \\
\text { tpr [\%] } \quad \text { fpr [\%] }\end{array}$} & \multicolumn{2}{|c|}{$\begin{array}{l}\text { NEO do sinal } \\
\text { tpr [\%] } \quad \text { fpr [\%] }\end{array}$} & \multicolumn{2}{|c|}{$\begin{array}{l}\text { NEO do valor absoluto } \\
\operatorname{tpr}[\%] \quad \mathrm{fpr}[\%]\end{array}$} \\
\hline 5 & 98.92 & 8.163 & 97.50 & 0.023 & 97.47 & 0.023 \\
\hline 10 & 98.78 & 4.536 & 98.13 & 0.212 & 98.07 & 0.213 \\
\hline 15 & 97.93 & 3.224 & 97.35 & 0.603 & 97.33 & 0.603 \\
\hline 20 & 95.39 & 1.308 & 96.89 & 1.157 & 96.83 & 1.157 \\
\hline 25 & 77.68 & 0.603 & 95.15 & 2.595 & 95.09 & 2.599 \\
\hline 30 & 46.07 & 0.358 & 91.37 & 3.510 & 91.37 & 3.499 \\
\hline 35 & 26.12 & 0.215 & 87.95 & 4.923 & 87.92 & 4.923 \\
\hline 40 & 14.29 & 0.214 & 84.52 & 6.260 & 84.49 & 6.241 \\
\hline
\end{tabular}
sinal e sobre o NEO do valor absoluto do sinal.

Tabela 4.1: Parâmetros de detecção: tpr (Itrue positive rate) e fpr (false positive rate)

Estes resultados mostram que praticamente não há diferencia entre aplicar o NEO diretamente no sinal ou ao valor absoluto do sinal. 


\subsection{Estratégia para realizar o operador Teager baseada no uso de circuitos elevadores ao quadrado}

No capítulo 3 estudou-se o operador não linear de energia conhecido como Teager ou NEO, cuja fórmula é a seguinte:

$$
\psi\left[x_{n}\right]=x_{n}^{2}-x_{n+1} \times x_{n-1}
$$

Nesta seção apresenta-se uma estratégia para realizar essa operação sobre correntes elétricas $I_{n-1}, I_{n}$ e $I_{n+1}$ que resultam da conversão a corrente de amostras analógicas de um sinal de tensão. O objetivo é gerar uma corrente de saída $I_{\text {neo }}$ de acordo com a seguinte expressão:

$$
I_{\text {neo }}=\frac{I_{n}^{2}}{I_{0}}-\frac{I_{n-1} \times I_{n+1}}{I_{0}}
$$

onde $I_{0}$ é uma constante com unidades de corrente elétrica. Para o caso da implementação com transistores MOS que será apresentada nas seções posteriores essa constante é a corrente de saturação sublimiar de um transistor PMOS.

A estratégia aqui apresentada permite a implementação dessa operação utilizando circuitos elevadores ao quadrado. Observando o primeiro termo do lado direito da fórmula (4.5) é evidente a utilização de um circuito elevador ao quadrado. Já a implementação mediante elevadores ao quadrado do segundo termo do lado direito dessa fórmula fica evidente observando a identidade apresentada a seguir:

$$
\frac{I_{n-1} \times I_{n+1}}{I_{0}}=\frac{\left(I_{n-1}+I_{n+1}\right)^{2}-I_{n-1}^{2}-I_{n+1}^{2}}{I_{0}}
$$

Aplicando essa identidade na fórmula (4.5) obtém-se a fórmula:

$$
I_{n e o}=\frac{I_{n-1}^{2}+2 I_{n}^{2}+I_{n+1}^{2}-\left(I_{n-1}+I_{n+1}\right)^{2}}{I_{0}}
$$

a qual implica que o operador NEO pode ser implementado utilizando 4 instâncias de um elevador ao quadrado. As somas e subtrações de correntes são implementadas facilmente aproveitando a lei de correntes de Kirchhoff. 


\subsection{Circuito elevador ao quadrado baseado em transis- tores MOS que operam na região sublimiar}

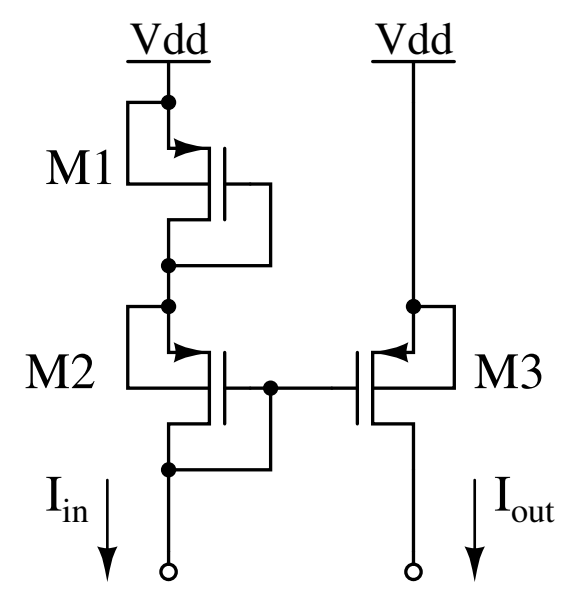

Figura 4.4: Circuito elevador ao quadrado. Considera-se entrada à corrente drenada do transistor M2 $\left(I_{\text {in }}\right)$ e como saída, à corrente fornecida pelo transistor M3 $\left(I_{\text {out }}\right)$

No circuito representado na figura 4.4, todos os transistores operam na região sublimiar na qual a corrente de dreno pode ser aproximada pela seguinte equação (MEAD, 1989), (TSIVIDIS, 1999), (LIU et al., 2002), (TSIVIDIS; MCANDREW, 2011):

$$
I_{d}=-I_{0} \exp \left(\frac{V_{s g}}{n \phi_{t}}\right)\left[1-\exp \left(-\frac{V_{s d}}{\phi_{t}}\right)\right]
$$

onde $I_{d}$ é a corrente que flui de dreno a fonte, $I_{0}$ é a corrente de saturação sublimiar, $n$ é fator de rampa e $\phi_{t}$ é a tensão térmica. Nessa equação as letras $\mathrm{g}, \mathrm{s}$ e d que aparecem nos subscritos representam os terminais de porta, fonte e dreno respectivamente. É importante lembrar que essa equação é válida quando os terminais de fonte e substrato estão interligados, como é o caso dos transistores representados na figura 4.4 .

Aplicando a fórmula (4.8) aos transistores M1, M2 e M3 da figura 4.4, os quais possuem as mesmas larguras de canal (W) e os mesmos comprimentos de canal (L), obtém-se: 


$$
\begin{gathered}
I_{\text {in }}=-I_{d 1}=I_{0} \exp \left(\frac{V_{s g 1}}{n \phi_{t}}\right)\left[1-\exp \left(-\frac{V_{s g 1}}{\phi_{t}}\right)\right] \\
I_{\text {in }}=-I_{d 2}=I_{0} \exp \left(\frac{V_{s g 2}}{n \phi_{t}}\right)\left[1-\exp \left(-\frac{V_{s g 2}}{\phi_{t}}\right)\right] \\
I_{\text {out }}=-I_{d 3}=I_{0} \exp \left(\frac{V_{s g 3}}{n \phi_{t}}\right)\left[1-\exp \left(-\frac{V_{s d 3}}{\phi_{t}}\right)\right]
\end{gathered}
$$

Para obter as equações (4.9) e (4.10) tem se aproveitado o fato de que nos transistores M1 e M2 as portas estão ligadas aos drenos. Observando as equações (4.9) e (4.10), conclui-se que $V_{s g 1}=V_{s g 2}$. Por outro lado, da figura 4.4 observa-se que $V_{s g 3}=V_{s g 1}+V_{s g 2}$. Combinando esses resultado com a equação (4.11), obtém-se:

$$
\begin{aligned}
I_{\text {out }} & =I_{0} \exp \left(\frac{2 V_{s g 1}}{n \phi_{t}}\right)\left[1-\exp \left(-\frac{V_{s d 3}}{\phi_{t}}\right)\right] \\
\Rightarrow I_{\text {out }} & =\frac{I_{\text {in }}^{2}}{I_{0}} \frac{\left[1-\exp \left(-\frac{V_{s d 3}}{\phi_{t}}\right)\right]}{\left[1-\exp \left(-\frac{V_{s g 1}}{\phi_{t}}\right)\right]^{2}}
\end{aligned}
$$

Para o caso em que $V_{s d 3}$ e $V_{s g 1}$ sejam muito maiores que $\phi_{t}$, o segundo fator da equação 4.13 é aproximadamente 1 e portanto:

$$
I_{\text {out }} \approx \frac{I_{\text {in }}^{2}}{I_{0}}
$$

Por exemplo, se $V_{s d 3}$ e $V_{s g 1}$ são maiores que $4 \phi_{t}$, o erro em se aproximar (4.13) por (4.14) é menor a $1 \%$.

Observando o resultado obtido (equação 4.14), deve-se notar que este circuito apresenta como desvantagem em relação ao elevador ao quadrado clássico baseado no princípio translinear a dependência do parâmetro de processo $I_{0}$. Porém para a aplicação que será dada a este circuito, essa dependência é facilmente compensada em nível de sistema. Por outro lado, o circuito aqui apresentado tem como principal vantagem o reduzido número de dispositivos. Além desses dois aspectos ambas soluções, aqui apresentada e a baseada no princípio translinear, compartilham outras virtudes e problemas. Por exemplo, em ambos casos existe susceptibilidade a variações locais de parâmetros que trazem como consequência um efeito conhecido como mismatch ou descasamento entre dispositivos. Posteriormente será analisado o problema de mismatch no contexto da aplicação dada ao elevador ao quadrado no 
sistema de detecção de impulsos elétricos.

De acordo com o desenvolvimento analítico apresentado, e deixando de lado por um momento o problema de mismatch, pode-se notar que a precisão do circuito no sentido de quão bem a saída se aproxima do quadrado da entrada depende de quão bem as correntes de dreno se aproximam de uma função exponencial da tensão portafonte. Por sua vez, essa dependência exponencial é mais exata quando o nível de inversão do canal do transistor é menor. Mediante um analise simplificada, sabe-se que para um dado valor de corrente de dreno e um dado valor de comprimento de canal (L) o nível de inversão diminui quando a largura de canal (W) aumenta. Então, em princípio, aumentando a largura de canal (W) dos transistores podemos melhorar a precisão do elevador ao quadrado. Porém, efeitos de segunda ordem tais como a presença de correntes de fuga impõem um limite a essa melhora.

Por meio de simulações elétricas pode-se determinar um valor apropriado de largura de canal (W). Para isso é necessário definir um parâmetro que meça a exatidão do elevador ao quadrado. Dado que no sistema de detecção de impulsos elétricos neurais o elevador ao quadrado processará valores de corrente dentro de uma determinada faixa (de $5 \mathrm{nA}$ a $20 \mathrm{nA}$ ), tal parâmetro deve ser calculado nessa faixa de corrente. Definimos como parâmetro de medida da precisão do elevador ao quadrado o coeficiente de correlação linear $(r)$ entre a corrente de saída obtida por simulação e o quadrado da corrente de entrada quando esta varia entre 5nA e 20nA.

Na figura 4.5 representa-se o diagrama esquemático utilizado para simular o circuito elevador ao quadrado e determinar a dependência do parâmetro de precisão escolhido $(r)$ e a largura de canal $(W)$. Nessa simulação, para cada valor de W escoIhido se aplicam uma serie de valores de corrente do intervalo [5nA, 20nA] e se medem as correspondentes correntes de saída. Com base nos vetores de corrente de entrada e saída, calcula-se o valor de ( $r$ ) correspondente ao valor de (W) escolhido. Esse procedimento se repete para outros valores de $(\mathrm{W})$ e finalmente pode se obter uma curva rvs. W.

Note-se que a tensão de saída é mantida fixa embora na prática a saída é ligada a um espelho de corrente de transistores NMOS através de um transistor PMOS configurado em base comum. Como será comprovado nas seções seguintes as variações da tensão de saída do elevador ao quadrado na configuração mencionada no parágrafo anterior são muito pequenas. 


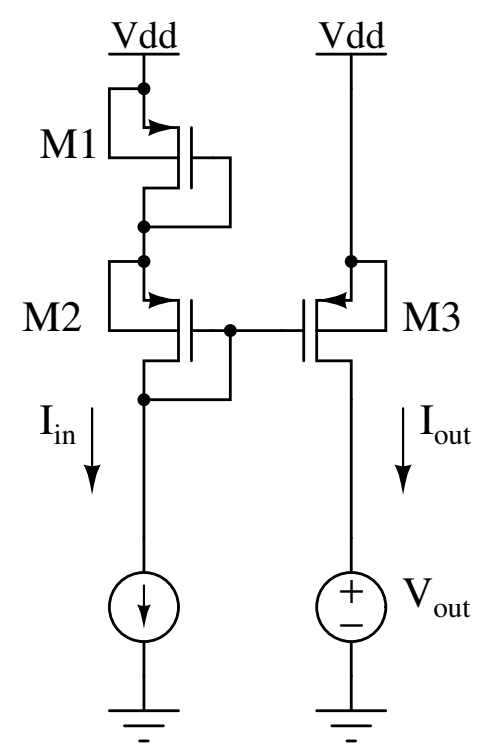

Figura 4.5: Circuito empregado para simular o elevador ao quadrado e determinar um valor apropriado de largura de canal (W)

Na figura 4.6 apresentam-se as curvas $r$ vs. W para três casos de conjunto de parâmetros de processo: typical, slow e fast. Como pode se observar nestes gráficos, a precisão do circuito não melhora de maneira significativa para valores de $\mathrm{W}$ maiores a $60 \mu \mathrm{m}$.

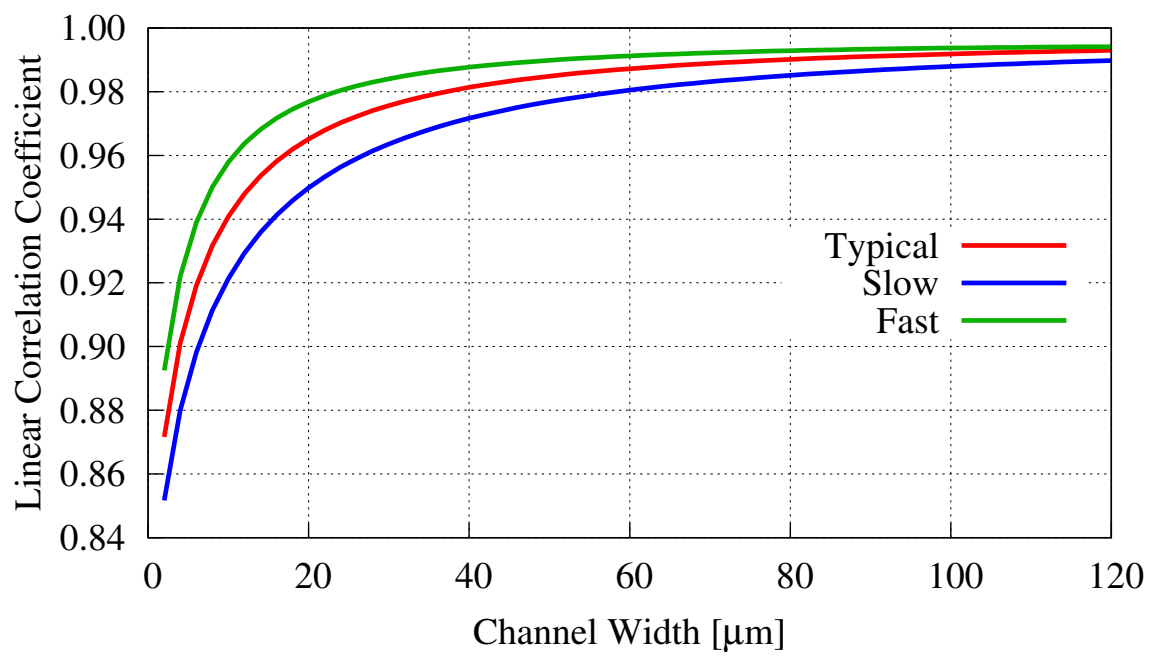

Figura 4.6: Coeficiente de correlação linear entre a corrente de saída, obtida mediante a simulação do circuito da figura 4.5, e o quadrado da corrente de entrada em função do comprimento de canal dos transistores PMOS. O comprimento de canal foi fixado em $1 \mu \mathrm{m}$. 


\subsection{Circuito que realiza o operador Teager em modo corrente}

O circuito proposto para a implementação do operador NEO é apresentado na figura 4.7. O circuito processa correntes de entrada representadas como $I_{n-1}, I_{n}$ e $I_{n+1}$ as quais são aplicadas a transistores NMOS. A saída do circuito é a corrente representada como $I_{\text {out }}$.

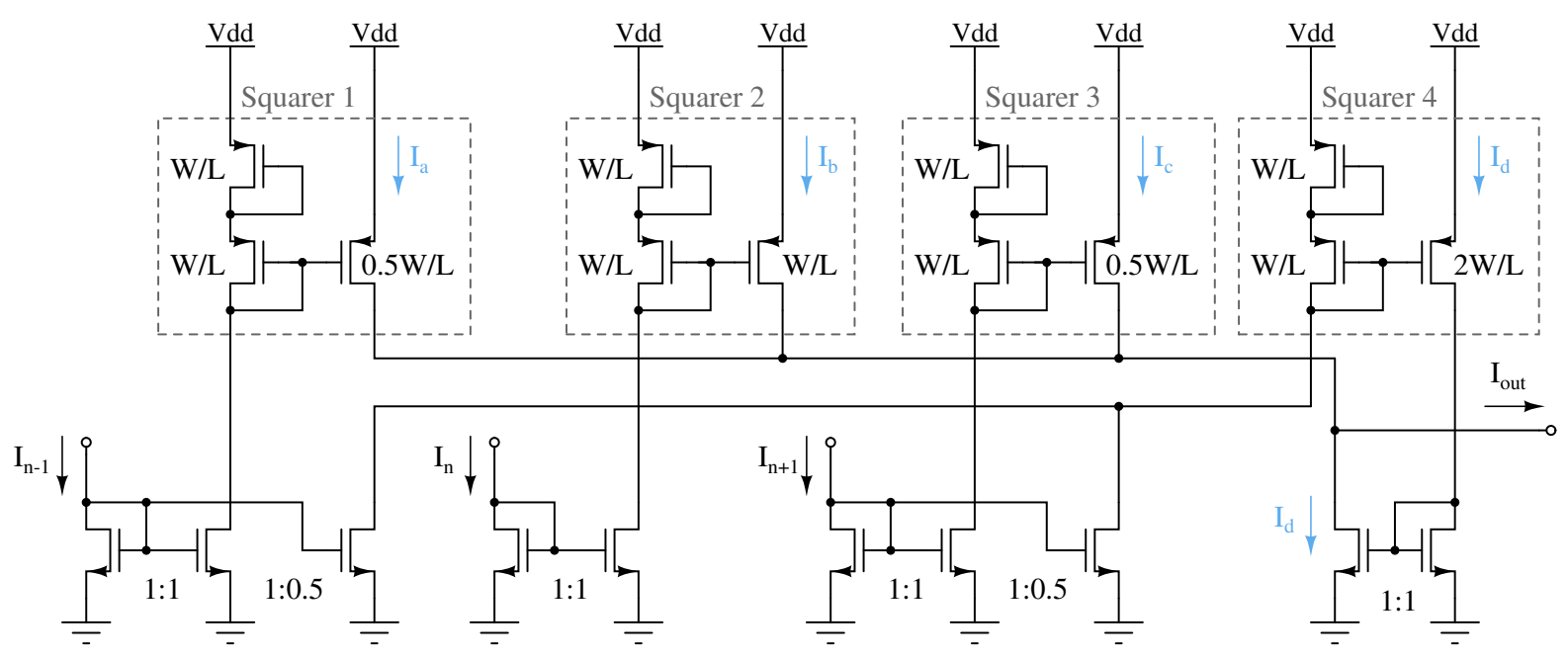

Figura 4.7: Esquema elétrico do circuito Operador NEO formado por elevadores ao quadrado do tipo mostrado na figura 4.4.

Para um correto funcionamento do circuito, as correntes de entrada devem ser aplicadas por circuitos com impedância de saída suficientemente maior que a impedância de entrada dos transistores NMOS que estão configurados como diodo. A impedância destes últimos é dada pelo inverso da sua transcondutancia. Os circuitos que aplicam as correntes são conversores de tensão a corrente iguais ao apresentado na seção 4.5. A impedância de saída do conversor tensão a corrente é da ordem da impedância de saída de uma fonte de corrente cascode, que é igual ao produto do ganho intrínseco de um transistor NMOS vezes a impedância de saída de outro transistor NMOS. Claramente a condição imposta no início deste parágrafo é cumprida (RAZAVI, 2001).

A seguir prova-se que o circuito da figura 4.7 aproxima a seguinte equação:

$$
I_{\text {out }}=\frac{I_{n}^{2}}{I_{0}}-\frac{I_{n-1} \times I_{n+1}}{I_{0}}
$$

Como mostrado na figura 4.7, o circuito está composto por 4 elevadores ao qua- 
drado. Aplicando a equação 4.14 aos três primeiros elevadores ao quadrado:

$$
\begin{aligned}
I_{a} & =\frac{I_{n-1}^{2}}{2 I_{0}} \\
I_{b} & =\frac{I_{n}^{2}}{I_{0}} \\
I_{c} & =\frac{I_{n+1}^{2}}{2 I_{0}}
\end{aligned}
$$

Ignorando o efeito de modulação de canal, a entrada do quarto elevador ao quadrado é $\frac{I_{n-1}}{2}+\frac{I_{n+1}}{2}$. O transistor de saída desse circuito possui o dobro de largura de canal que o transistor de entrada, portanto a corrente de saída é o dobro do quadrado da corrente de entrada dividido por $I_{0}$ :

$$
I_{d}=\frac{\left(I_{n-1}+I_{n+1}\right)^{2}}{2 I_{0}}
$$

Aplicando a lei de correntes de Kirchhoff ao nó de saída:

$$
I_{\text {out }}=I_{a}+I_{b}+I_{c}-I_{d}
$$

Substituindo (4.16), (4.17), (4.18) e (4.19) na equação (4.20) obtém-se a seguinte relação:

$$
I_{\text {out }}=\frac{I_{n}^{2}}{I_{0}}+\frac{I_{n-1}^{2}}{2 I_{0}}+\frac{I_{n+1}^{2}}{2 I_{0}}-\frac{\left(I_{n-1}+I_{n+1}\right)^{2}}{2 I_{0}}
$$

a qual, após algumas simplificações, fica igual à equação (4.15). 


\subsection{Circuito que gera uma corrente proporcional ao va- lor absoluto do sinal de tensão de entrada}

O circuito conversor de tensão a corrente desenvolvido nesta tese baseia-se no mesmo princípio que o circuito reportado em (LÓPEZ-MARTín et al., 2011) que consiste em aplicar a tensão de entrada a um dos terminais de um resistor cujo outro terminal está ligado a uma terra virtual gerada pela realimentação negativa de um amplificador operacional. A figura 4.8 representa esse princípio.

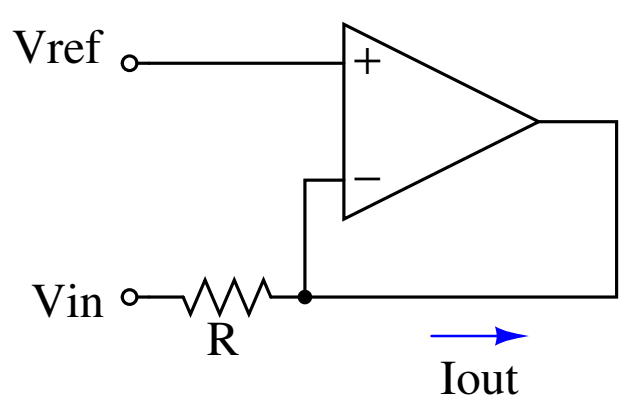

Figura 4.8: Princípio de operação do conversor de tensão a corrente

Nessa figura Vin é a tensão de entrada que representa a atividade neuronal. Essa tensão é o resultado da amplificação e filtragem que são os realizados nos estágios iniciais de um canal de registro neural. Esse sinal deve vir com um nível de referência igual a Vref. Adotou-se que a amplitude pico-pico máxima do sinal de entrada seja de $40 \mathrm{mV}$, embora o conversor de tensão a corrente projetado admita amplitudes maiores. A corrente de saída é:

$$
\text { Iout }=\frac{\text { Vin }-V r e f-v o s}{R}
$$

onde vos é a tensão de offset do amplificador operacional que é da ordem de $\mathrm{mV}$ e introduz um erro na corrente de saída. Para poder aplicar a saída desse conversor a um elevador ao quadrado é necessários transladá-la a um pino de saída de alta impedância. Além do mais, precisa-se entregar apenas o valor absoluto do sinal. Para conseguir isso se implementaram espelhos de corrente que medem a corrente no estágio de saída do amplificador operacional, como representado na figura 4.9. 
Estágio de saída

do opamp

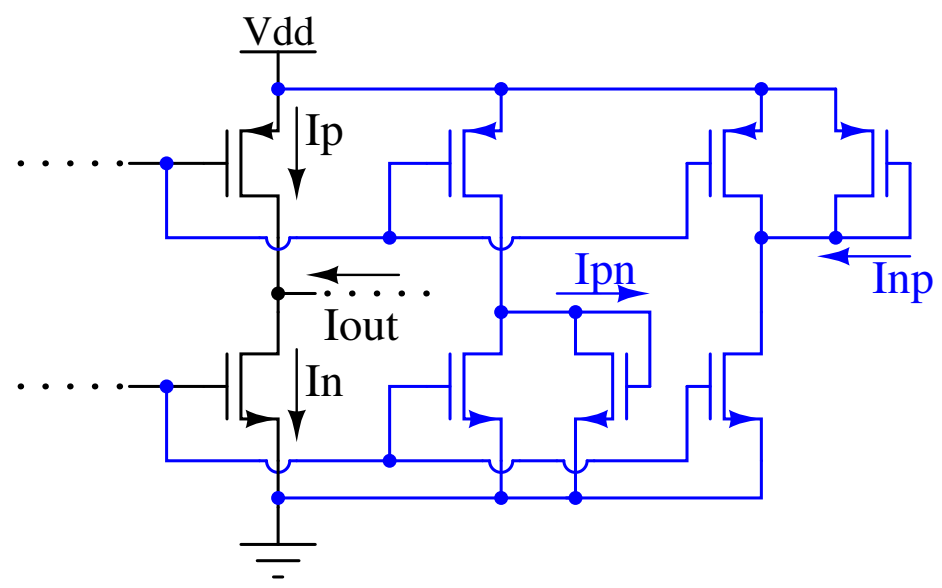

Figura 4.9: Estratégia para gerar valor absoluto da corrente Iout

Nessa figura os transistores em cor preta compõem o estágio de saída do amplificador operacional. Então a corrente de saída, no sentido especificado nas figuras 4.8 e 4.9 é igual a $I n-I p$.

O objetivo é gerar uma corrente igual a:

$$
\text { Iabs }=\mid \text { Iout }|=| \text { Ip }- \text { In } \mid
$$

Devido à copias realizadas pelos transistores em azul, a corrente Ipn será igual a Ip - In apenas quando Ip for maior que In, e em caso contrário será 0. Similarmente Inp será igual a In - Ip apenas quando In seja maior a Ip, e em caso contrário será igual a 0 . Em conclusão, o valor absoluto de corrente pode ser obtido somando essas componentes:

$$
I a b s=I p n+\operatorname{Inp}
$$

essa soma é obtida facilmente mediante espelhos de corrente. 


\subsection{Simulações elétricas do sistema de detecção de- senvolvido}

O operador não linear de energia (NEO) é uma alternativa para o estágio de préênfase em sistemas de detecção de impulsos elétricos neurais. Essa tarefa consiste em enfatizar a diferença entre os spikes neurais é o ruido de fundo. Para avaliar o desempenho do circuito em termos dessa capacidade de pré-ênfase, utilizamos os dado sintéticos apresentados no capítulo 1 (QUIROGA; NADASDY; BEN-SHAUL, 2004).

É necessário avaliar o circuito com dados sintéticos pois dessa maneira podemos conhecer exatamente em que instantes acontecem os spikes e assim verificar se o circuito os enfatiza em relação ao ruído.

Na figura 4.10(a) apresenta-se um intervalo de 200ms de um dos sinais disponíveis no banco de dados escolhido. Utiliza-se o símbolo '+' para identificar a presença de spikes. Nesse sinal o desvio padrão do ruído é igual ao $25 \%$ da amplitude máxima.

A resposta do circuito ao sinal escolhido é mostrado na figura 4.6(b). Visualmente é possível verificar que o circuito atenuou o ruído ao mesmo tempo que amplificou os spikes.

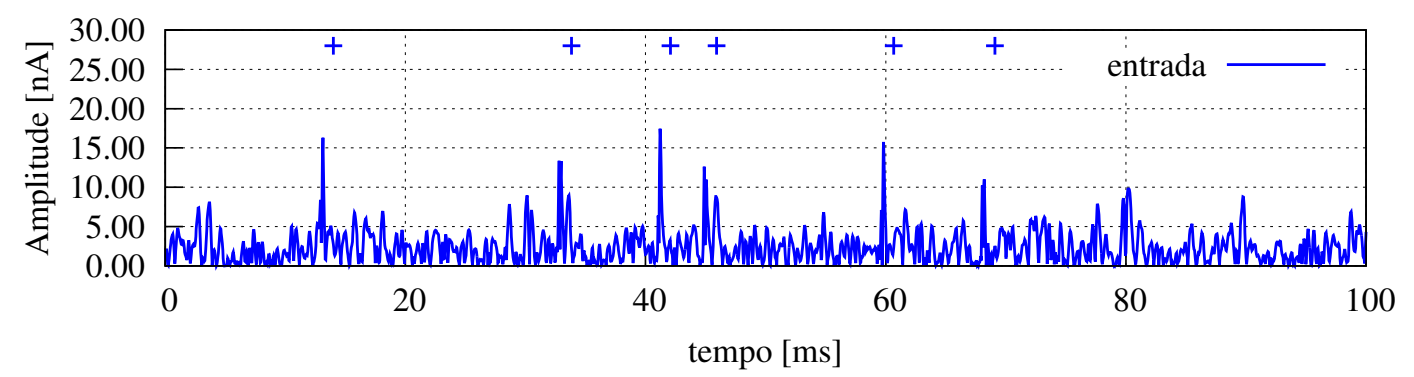

(a)

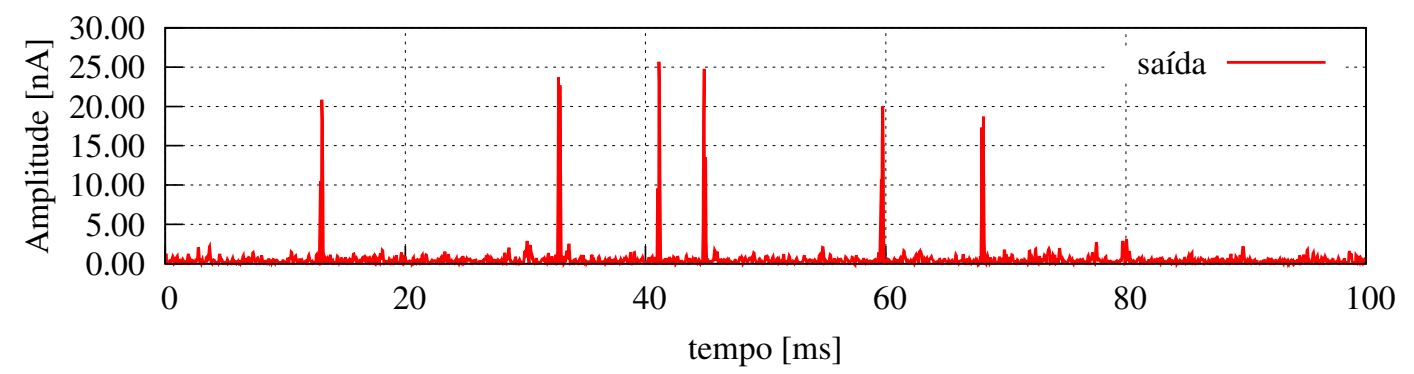

(b)

Figura 4.10: (a) Sinal sintético aplicado ao circuito NEO. O desvio padrão do ruído é igual ao $25 \%$ da amplitude máxima do sinal. (b) Resposta do circuito NEO à aplicação do sinal sintético escolhido. 
Apesar de ficar evidente por inspeção visual da figura 4.10, o desempenho do circuito precisa ser avaliado por métodos mais objetivos. Um desses métodos consiste na construção da curva ROC (Receiver Operating Curve).

$\mathrm{Na}$ figura 4.11 apresenta-se uma ROC que relaciona a taxa de verdadeiros positivos vs a taxa de falsos positivos dada uma variação do limiar de comparação. A taxa de de verdadeiros positivos foi calculada dividindo a quantidade de spikes corretamente detectados pela quantidade total de spikes. A taxa de falsos positivos foi calculada dividindo a quantidade de vezes que o ruido foi considerado como spike pela quantidade total de spikes que haveriam no tempo em que de fato não acontecem spikes. Para essa última parte da conta a duração de um spike foi considerada como $2 \mathrm{~ms}$.

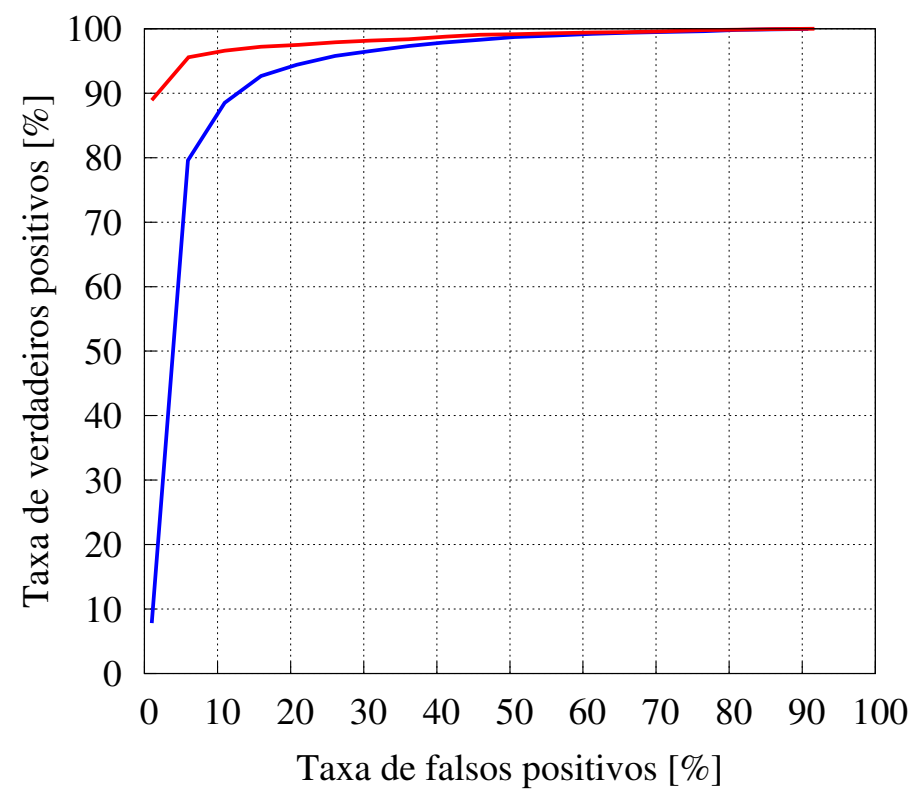

Figura 4.11: Curva ROC para o sinal original (linha em azul) e para o sinal de saída do circuito NEO (linha em vermelho).

Comparando a curva ROC do sinal original com a da saída do circuito NEO nota-se a capacidade do circuito NEO para enfatizar a presença de spikes. Essa capacidade permite uma melhor separação de spikes do ruído.

A tabela 4.2 lista parâmetros que quantificam a detecção de spikes no sinal original e no sinal de saída do circuito NEO. Os parâmetros apresentados são a taxa de verdadeiros positivos (TPR de True Positve Rate) e a taxa de falsos positivos (FPR de False Positive Rate). Quanto maior o nível de ruído a melhora na separabilidade dos spike através do circuito NEO mais evidente fica. 
Tabela 4.2: Taxas de detecção para diferentes níveis de ruído. O limiar de comparação foi fixado em $8 \mathrm{nA}$.

\begin{tabular}{|c|cc|cc|}
\hline \multirow{2}{*}{ Ruído } & \multicolumn{2}{|c|}{ Sinal Original } & \multicolumn{2}{c|}{ Saída do circuito NEO } \\
\cline { 2 - 5 } & TPR & FPR & TPR & FPR \\
\hline $5 \%$ & $88,2 \%$ & $0,27 \%$ & $100 \%$ & $0,45 \%$ \\
\hline $10 \%$ & $82,3 \%$ & $0,18 \%$ & $100 \%$ & $0,40 \%$ \\
\hline $15 \%$ & $80,0 \%$ & $0,15 \%$ & $99 \%$ & $0,40 \%$ \\
\hline $20 \%$ & $49,4 \%$ & $0,10 \%$ & $99,5 \%$ & $0,30 \%$ \\
\hline
\end{tabular}

O circuito foi avaliado também aplicando um sinal real humano. O sinal corresponde a 30 minutos de registro de um paciente epiléptico realizado no laboratório do professor Itzhak Fried da Universidade da Califórnia Los Angeles (UCLA), utilizando um sistema da empresa Neuralynx (QUIROGA; NADASDY; BEN-SHAUL, 2004). Na figura 4.12 a linha em cor azul é um segmento de $9 \mathrm{~ms}$ desse sinal, contendo um spike. A resposta do circuito NEO à aplicação desse sinal é representada mediante a linha em vermelho. O sinal de saída foi escalado de forma que os pico máximo coincida com o pico máximo de sinal de entrada. Observa-se claramente a melhora na qualidade do sinal.

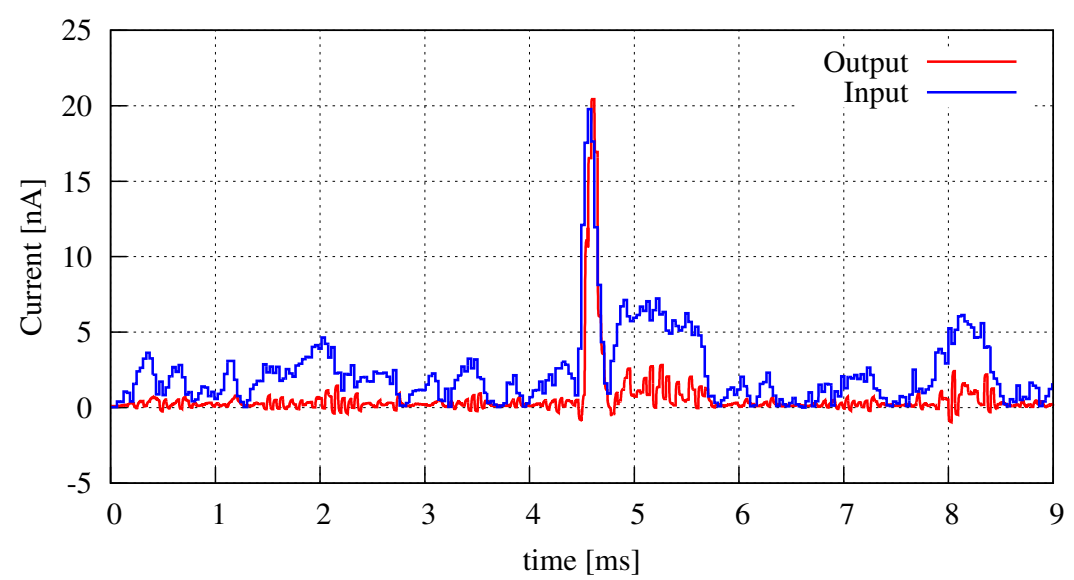

Figura 4.12: Resposta do circuito a um sinal real. O sinal de saída foi escalado de maneira que o pico máximo coincida com o pico máximo do sinal de entrada.

Mediante as simulações foi comprovado que o circuito NEO gasta 300 pJ para processar todas as amostras de um spike.

O trabalho apresentado neste capítulo deu como resultado as seguintes publicações (SALDAÑA-PUMARICA; DEL-MORAL-HERNANDEZ, 2016a), (SALDAÑAPUMARICA; DEL-MORAL-HERNANDEZ, 2016b). 
Na tabela 4.3 apresenta-se uma comparação deste trabalho com trabalhos similares encontrados na literatura.

Tabela 4.3: Comparação com outros trabalhos

\begin{tabular}{|c|c|c|c|c|c|c|c|}
\hline Referência & $\begin{array}{l}\text { Congresso } \\
\text { Periódico } \\
\end{array}$ & $\begin{array}{c}\text { Processo } \\
{[\mathrm{nm}]}\end{array}$ & $\begin{array}{c}\text { Área } \\
{\left[\mathrm{mm}^{2}\right]} \\
\end{array}$ & $\begin{array}{l}\text { VDD } \\
{[\mathrm{V}]} \\
\end{array}$ & $\begin{array}{c}\text { Potência } \\
{[\mu \mathrm{W}]} \\
\end{array}$ & método & $\begin{array}{c}\text { Estilo de } \\
\text { Implementação } \\
\end{array}$ \\
\hline (HOLLEMAN et al., 2008) & CICC 2008 & 130 & 0.044 & 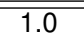 & 0.95 & NEO & Analógico \\
\hline $\begin{array}{l}\text { (KOUTSOS; PARASKEVOPOULOU; } \\
\text { CONSTANDINOU, 2013) }\end{array}$ & ISCAS 2013 & 180 & 0.030 & 1.8 & 1.50 & NEO & Analógico \\
\hline (LI et al., 2013) & ISCAS 2013 & 130 & - & 0.5 & 0.26 & fNEO & Analógico \\
\hline (RODRÍGUEZ-PÉREZ et al., 2012) & TBCAS 2012 & 130 & 0.160 & 1.2 & 0.20 & Limiar & Analógico \\
\hline (LAPOLLI; COPPA; HÉLIOT, 2013) & EMBC 2013 & 65 & 0.017 & - & 1.83 & NEO & Digital \\
\hline $\begin{array}{l}\text { Esta tese (SALDAÑA-PUMARICA; } \\
\text { DEL-MORAL-HERNANDEZ, 2016b) }\end{array}$ & EPUSP & 90 & 0.040 & 1.2 & 0.30 & NEO & Analógico \\
\hline
\end{tabular}

As informações faltantes nessa tabela (indicadas por "-") não são fornecidas nas respectivas referências, (LI et al., 2013) e (LAPOLLI; COPPA; HÉLIOT, 2013).

Ao comparar o consumo de potência é importante considerar o efeito da tensão de alimentação. 


\section{Sistema de classificação de impulsos elétricos neurais}

Este capítulo trata do desenvolvimento e simulação de um sistema de classificação de impulsos elétricos neurais spikes presentes nos sinais coletados por eletrodos implantados no córtex. O sistema foi projetado visando a sua integração numa interface implantável de registro e transmissão sem fio de sinais neurais extracelulares. Uma das características mais importantes do sistema, que constitui o principal aporte deste trabalho, é a representação de amostras de spikes mediante atrasos entre pulsos digitais. Essa representação é a adotada num tipo de processamento conhecido como processamento de sinais em modo tempo ou em inglês Time-Mode Signal Processing (RAVINUTHULA et al., 2009), (GUTTMAN; ROBERTS, 2009). O processamento de sinais em modo tempo vem sendo explorado por vários pesquisadores em diversos contextos com o propósito de atenuar as consequências adversas da redução da tensão de alimentação em tecnologias de fabricação modernas (TAILLEFER; ROBERTS, 2009), (DHANASEKARAN et al., 2011), (ZHU; YUAN; KHAN, 2013), (VIGRAHAM; KUPPAMBATTI; KINGET, 2014), (UNNIKRISHNAN; VESTERBACKA, 2014).

O sistema desenvolvido executa a classificação com base no formato dos spikes de uma maneira similar à como operam os sistemas de Template Matching. Como discutido nos capítulos iniciais a classificação de spikes com base nos formatos é uma das alternativas empregadas no processo de spike sorting. O sistema aqui apresentado foi desenvolvido considerando a sua aplicação específica em sistemas implantáveis de spike sorting. O alinhamento dos spikes de entrada é um procedimento importante dentro de um sistema de Spike Sorting. Neste trabalho não foi abordado esse procedimento, mas é uma proposta de trabalho futuro importante para complementar o sistema de classificação aqui apresentado.

O classificador desenvolvido recebe como entrada um conjunto de amostras de um spike e as compara com as amostras de protótipos previamente definidas. Um 
protótipo é um conjunto de amostras de um spike que considera-se representante de uma determinada classe (a classe de spikes gerados por um mesmo neurônio). A comparação consiste no cômputo da distância de Manhattan entre o conjunto de amostras de entrada e os conjuntos de amostras dos protótipos. A menor das distâncias corresponde à classe à qual pertence o spike de entrada. Para o cômputo da distância de Manhattan implementou-se uma estratégia baseada na representação de amostras como atrasos entre pulsos digitais.

Foram realizadas simulações considerando três protótipos, porém o sistema pode ser estendido para classificar spikes em mais de três classes. De maneira simplificada, o sistema pode ser representado como na figura 5.1 .

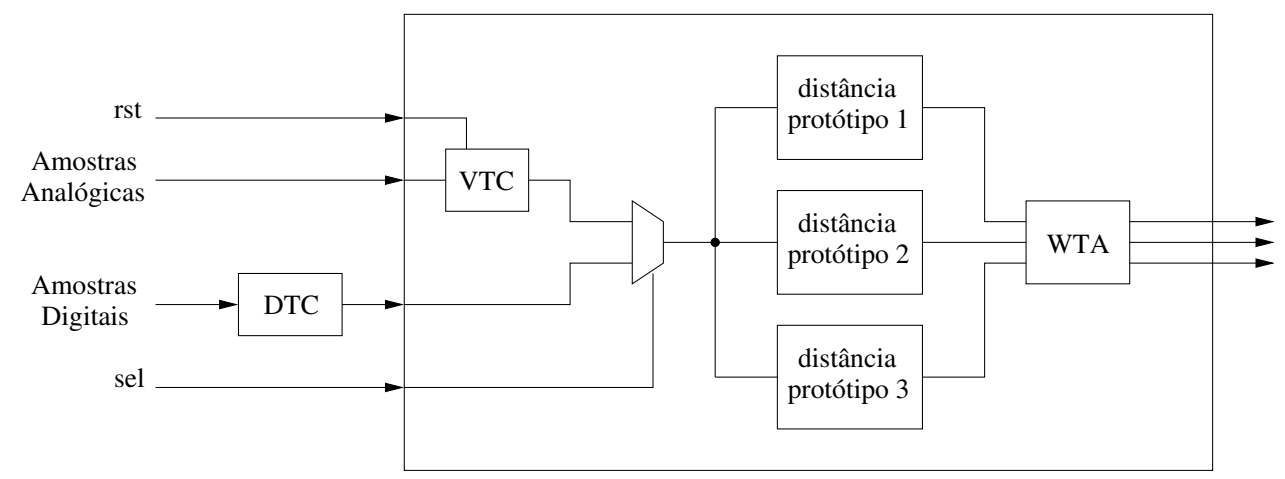

Figura 5.1: Diagrama de blocos simplificado do sistema de classificação de spikes desenvolvido. VTC é o conversor de tensão a tempo (Voltage-to-Time Converter), WTA é o circuito que determina qual das três entradas é menor (Winner Take All).

O sistema pode receber amostras analógicas de um spike de maneira serial. Internamente o sistema converte as amostras analógicas em pulsos digitais através de um conversor de tensão a tempo (VTC de Voltage-to-Time Converter). A informação está contida nos atrasos desses pulsos em relação a um sinal digital periódico de referência que deve ser aplicado na entrada rst. Esse sinal recebe esse nome (rst) porque serve para descarregar (resetar) um capacitor interno.

O sistema também pode operar em um contexto em que estejam disponíveis amostras digitais do spike que se deve classificar. Para isso é necessário um conversor DTC (Digital to Time Converter). Neste trabalho não foi abordado o projeto do circuito DTC.

Para determinar a qual classe pertence o spike de entrada, calcula-se a distância de Manhattan entre o spike e os protótipos que representam cada classe. A menor das distâncias determinará a qual classe pertence o spike. A distância de Manhattan 
é mais fácil de implementar em hardware que a distância euclidiana. Outra vantagem do uso da distância de Manhattan é o melhor desempenho na classificação quando aumenta o ruído; isto foi mostrado mediante simulações em (KARKARE; GIBSON; MARKOVIC, 2013). A saída do circuito que calcula a distância é uma tensão proporcional à distância.

O circuito WTA (Winner Take All) determina qual das três tensões que representam distâncias foi menor e ativa uma das três saídas digitais indicando qual foi a classe identificada.

Em (KARKARE; GIBSON; MARKOVIC, 2013), apresenta-se um sistema de spike sorting onde os estágios de agrupamento e classificação são baseados na distância de Manhattan. Trata-se de uma implementação completamente digital onde os consumos de área e de potência são dominados pelos registradores que armazenam os protótipos. No sistema de classificação apresentado nesta tese a informação relativa aos protótipos é representada nos atrasos introduzidos por uns circuitos chamados de Delay Elements (DE). O armazenamento temporário desses atrasos depende da tensão em capacitores. Como será explicado nas seções a seguir essa metodologia permite reduzir tanto a área como a potência necessária para operar com os protótipos.

\subsection{Representação de sinais como atrasos entre pul- sos digitais}

Como mencionado nos parágrafos iniciais deste capítulo, a representação baseada em tempo é uma das características mais importantes do sistema de classificação proposto. No sistema proposto, a conversão de tensão analógica a tempo é realizada por um circuito chamado VTC (Voltage to Time Converter). O sistema pode operar diretamente com informação representada em tempo o que possibilita a utilização de amostras digitais as quais devem ser convertidas a tempo através de um circuito chamado DTC(Digital to Time Converter). Nas explicações a seguir assume-se que se trabalha com amostras analógicas, porém, são válidas para o caso digital desde que se substitua VTC por DTC.

Na figura 5.2 ilustra-se a representação de amostras como atrasos de pulsos digitais em relação a um instante de referência $\left(t_{r s t}\right)$. 

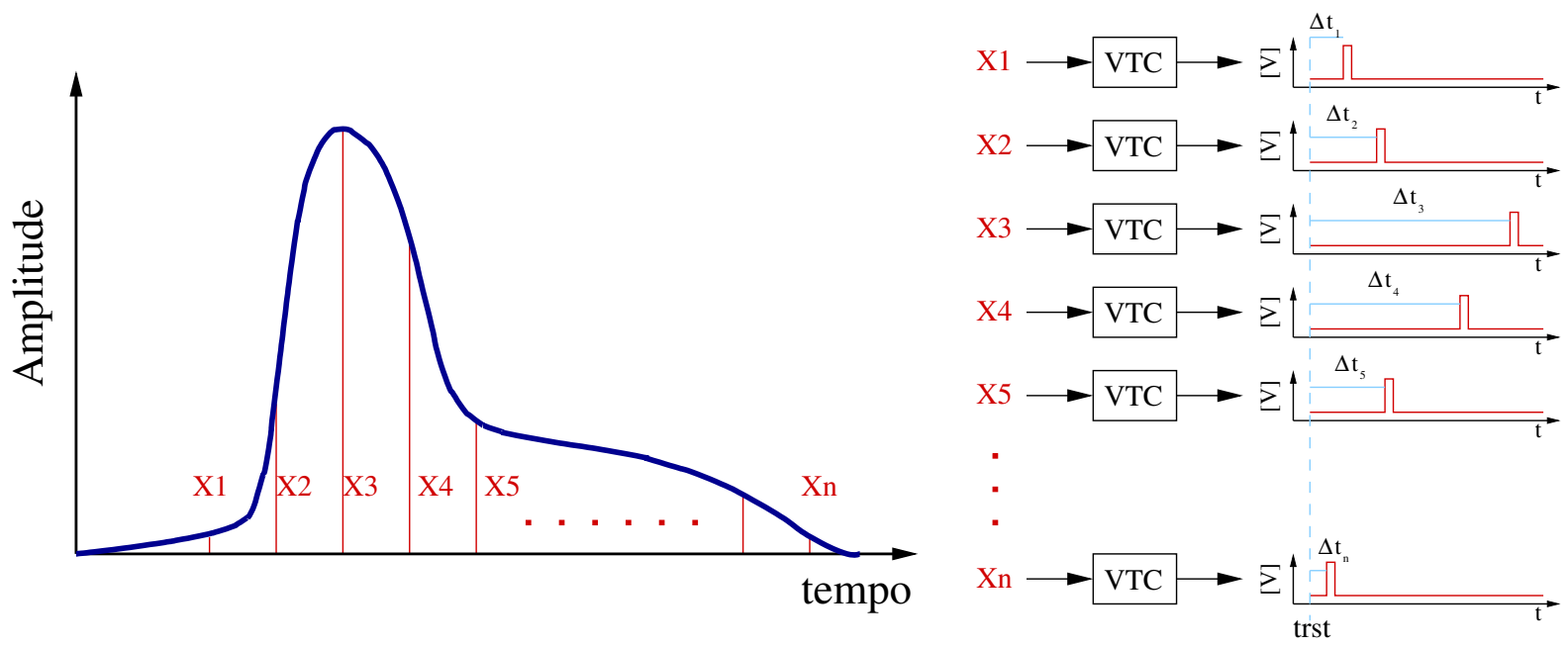

Figura 5.2: Representação de amostras baseada em tempo. Os atrasos dos pulsos em relação a $t_{r s t}$ são proporcionais às tensões de entrada dos VTC.

Nessa figura cada VTC converte uma amostra analógica de um spike em um pulsos digital atrasado em relação a $t_{r s t}$ um tempo proporcional à amostra. Na prática as amostras do spike são aplicadas sequencialmente portanto é suficiente utilizar um único VTC de maneira que dentro de cada período do sinal $t_{r s t}$ se terá um pulso com um atraso correspondente à a mostra analógica que está representando (ver figura 5.3.

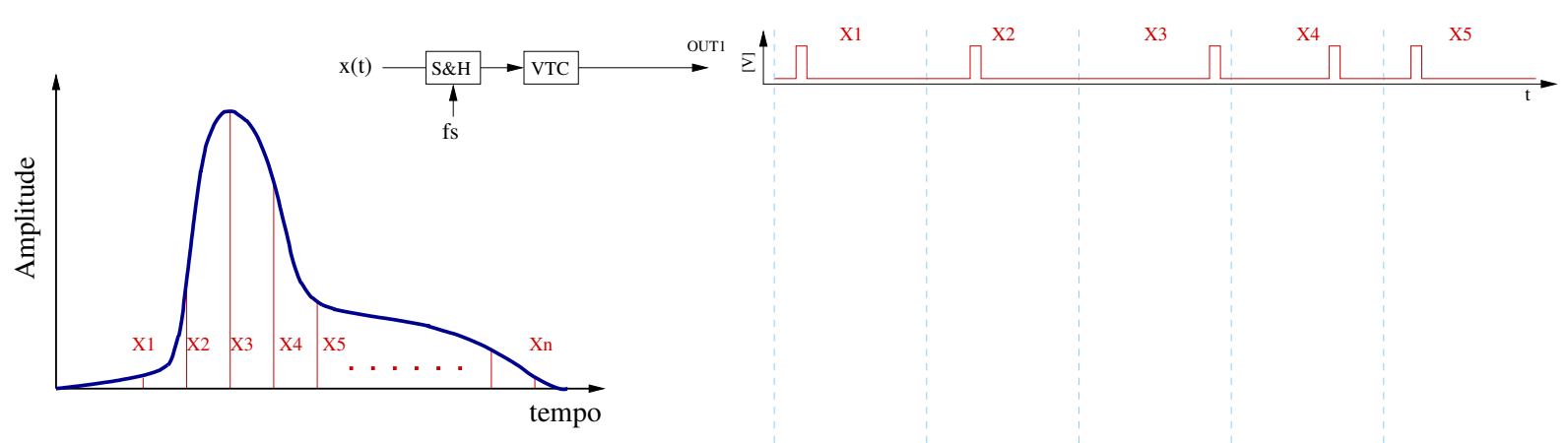

Figura 5.3: Representação de amostras baseada em tempo mediante um único VTC.

\subsection{Conversor de tensão a tempo (VTC)}

O circuito que realiza a conversão a tempo opera de maneira similar a um modulador tipo PWM (Pulse Width Modulator). Na figura 5.4 representa-se um circuito que realiza esse tipo de conversão. Esse é um dos circuitos mais clássicos para a conversão de tensão a tempo. 

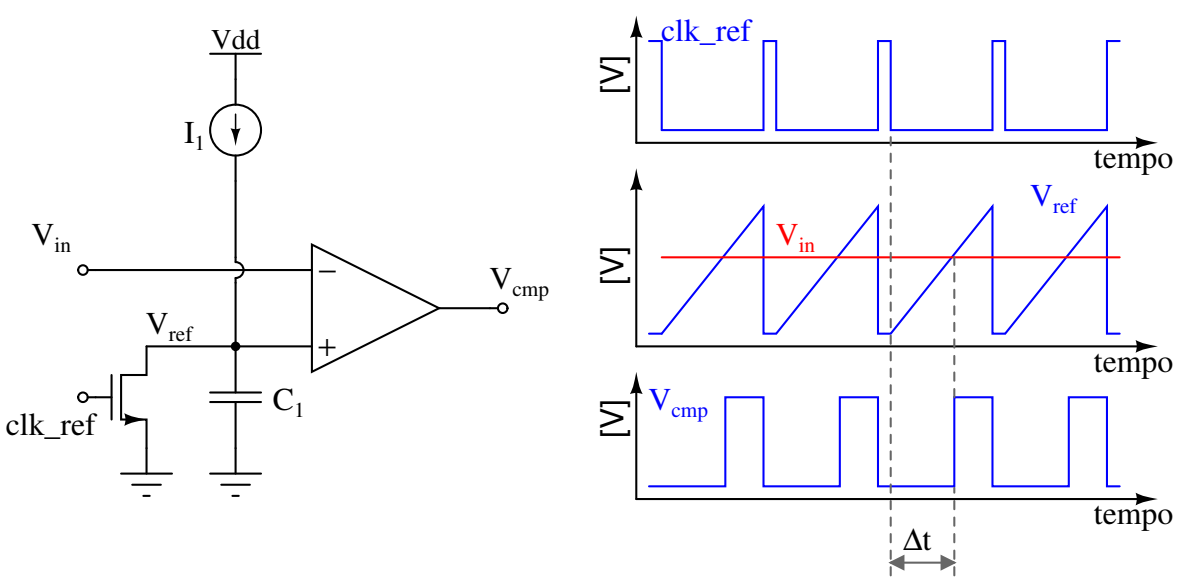

Figura 5.4: Conversor de tensão a tempo baseado em modulação PWM (Pulse Width Modulator.

Nessa figura $V_{i n}$ representa o valor amostrado do sinal que pretende-se processar. Embora a figura corresponda ao caso em que o sinal de entrada é constante, o resultado que será derivado a seguir é válido para um sinal variável que é amostrado utilizando o sinal $c l k \_r e f$. Após o capacitor ser resetado a saída do comparador demora um tempo $\Delta t$ para comutar:

$$
\Delta t=\frac{C_{1}}{I_{1}} \times V_{i n}
$$

Entre várias outras implementações encontradas na literatura escolheu-se uma que tem como vantagem em relação a alternativa clássica da figura 5.4 que uma das entradas do comparador é mantida fixa (MOHAMAD et al., 2013). O esquema de essa implementação é mostrado na figura 5.5.

Neste caso é válida a seguinte equação:

$$
\Delta t=\frac{C_{1}}{I_{1}} \times \Delta V
$$

Onde $\Delta V=V_{i n}-V_{\text {ref }}$. Um parâmetro que caracteriza ao circuito VTC é o fator de conversão que para o caso analisado é igual a:

$$
K_{V T C}=\frac{C_{1}}{I_{1}}
$$

É importante notar que existe uma relação entre o período de amostragem e a faixa de tensão que pode ser codificada. Para o cálculo da diferença entre amostras com representação de sinais baseada em tempo, necessário para a proposta 

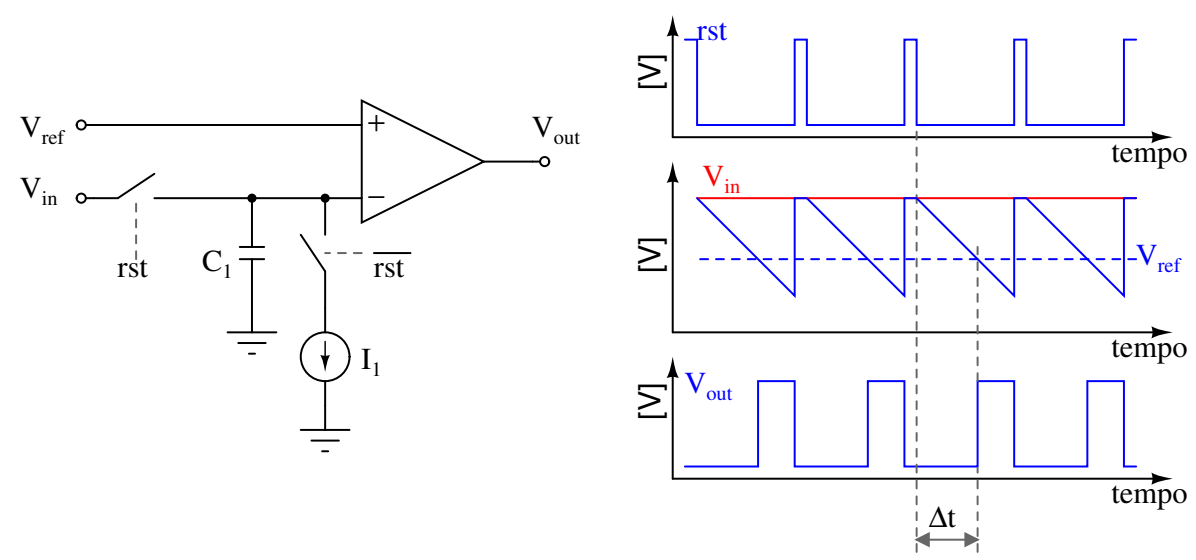

Figura 5.5: Conversor de tensão a tempo apresentado em (MOHAMAD et al., 2013). Neste caso uma das tensões de entrada do comparador é constante o qual facilita 0 seu projeto.

de cômputo da distância de Manhattan, parte do período de amostragem deve ser reservado para as operações. A sinais que serão utilizados nas simulações do sistema de classificação correspondem a uma taxa de amostragem de $24 \mathrm{kHz}$. Para as operações com representação baseada em tempo usaremos um terço do período para representação das amostras. Considerando uma corrente de descarga de 62,5nA e um capacitor de 2 pf teremos que a terceira parte do período de amostragens corresponde a:

$$
\begin{aligned}
\Delta V & =\frac{I}{C} \times \Delta t \\
& =\frac{I}{C} \times \frac{T_{\text {samp }}}{3} \\
& =\frac{I}{C} \times \frac{1}{3 f_{\text {samp }}} \\
& =\frac{62.5 n A}{2 p F} \times \frac{1}{3 \times 24 k H z} \\
& =434 m V
\end{aligned}
$$

Então nas simulações devemos considerar que a máxima amplitude pico-pico do sinal de entrada deve ser menor que $434 \mathrm{mV}$. Mediante simulações comprovou-se que o consumo médio do conversor é menor a 900 nW. 


\subsection{Cômputo da distância de Manhattan}

Nesta seção se detalha uma das propostas deste trabalho de doutorado. Trata-se da estratégia adotada para o cálculo de distância entre dois conjuntos de grandezas (padrões).

O sistema proposto recebe 30 amostras de tensão $\left\{x_{1}, x_{2}, \ldots\right.$ e $\left.x_{30}\right\}$, que chamaremos padrão de entrada e gera uma tensão de saída proporcional à distância de Manhattan entre esse conjunto de amostras e outro conjunto de amostras de tensão $\left\{y_{1}, y_{2}, \ldots\right.$ e $\left.y_{30}\right\}$ que chamaremos padrão de referência.

$$
D_{M}=K \sum_{i=1}^{30}\left|x_{i}-y_{i}\right|
$$

onde K é uma constante de proporcionalidade adimensional. As amostras podem ser aplicadas de maneira paralela ou de maneira sequencial, sendo a segunda alternativa a mais apropriada para o caso de sinais temporais como é o caso dos sinais neurais.

O elemento básico deste sistema é um circuito que gera uma tensão proporcional ao módulo da diferença entre um par de amostras $x_{i}$ e $y_{i}$ :

$$
d_{i}=K\left|x_{i}-y_{i}\right|
$$

Essa operação pode ser realizada de maneira simples adotando uma codificação baseada no tempo. O circuito que realiza essa operação será chamado de circuito subtrator baseado em tempo.

\subsubsection{Circuito subtrator baseado em tempo}

O esquema do circuito é mostrado na figura 5.6.

Nessa figura a amostra de entrada é $V_{x}$ e amostra de referência a $V_{y}$. O circuito opera em duas fases, na primeira fase o circuito é configurado para poder calcular diferenças em relação a uma determinado amostra de referência $V_{y}$. De certa maneira podemos considerar que na fase de configuração o circuito armazena ou aprende a amostra de referência $\left(V_{y}\right)$. Após essa fase o circuito está pronto para calcular a diferença entre qualquer amostra de entrada e amostra que foi armazenada ou apren- 


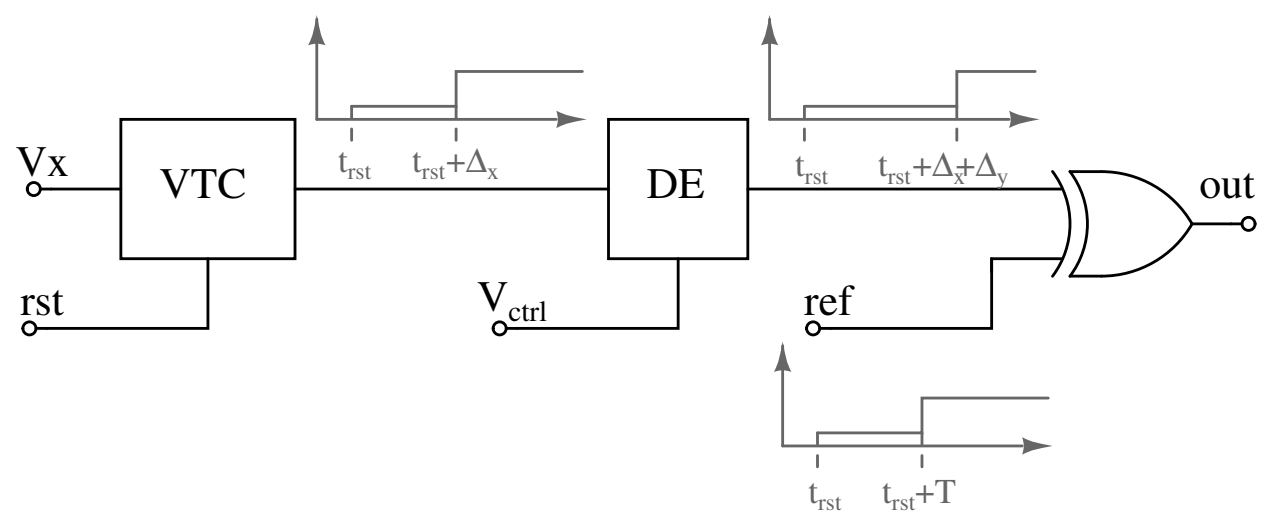

Figura 5.6: Circuito subtrator baseado em tempo. VTC: Voltage to Time Converter, DE: Delay Element.

dida. A segunda fase, então, é a fase de cálculo da diferença $\left(\left|V_{x}-V_{y}\right|\right)$.

A seguir se explica a fase de cálculo da diferença, e numa subsecção posterior será explicado como se realiza a configuração do circuito (aprendizado da amostra de referência $V_{y}$ ). A operação do circuito pode ser entendida com ajuda do diagrama de tempos da figura 5.7 .

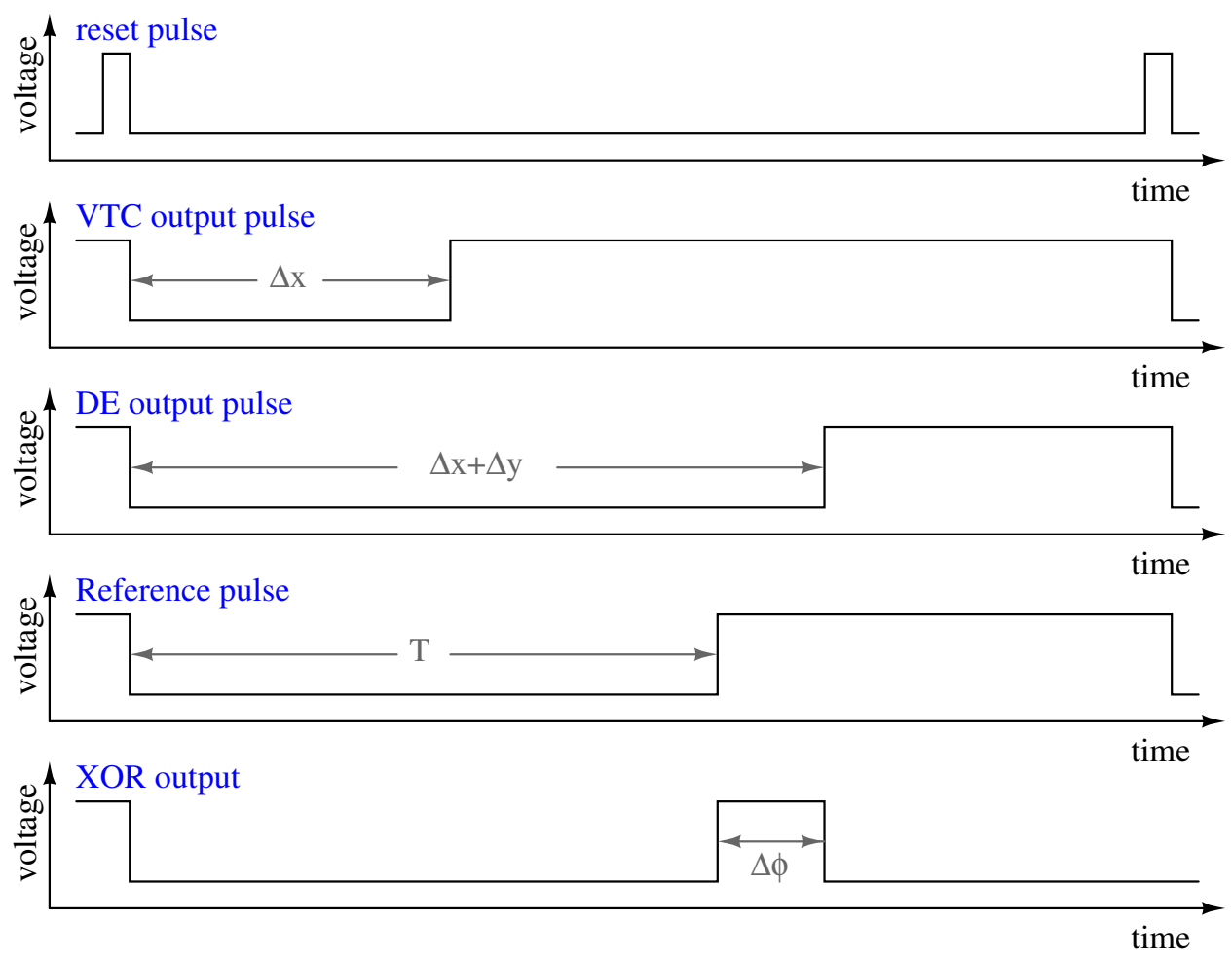

Figura 5.7: Diagrama de tempos da operação do circuito subtrator 
Quando acontece um pulso de reset inicia-se a conversão de tensão a tempo da amostra de entrada $\left(V_{x}\right)$. A saída do VTC comuta de nível baixo para nível alto um tempo $\Delta_{x}$ após a borda de descida do pulso de reset. De acordo com a explicação dada sobre o VTC se cumpre:

$$
\Delta_{x}=K_{V T C} \times V_{x}
$$

onde $K_{V T C}$ é o fator de conversão do VTC, dado pela equação 5.3.

O circuito DE (Delay Element) introduz um atraso $\Delta_{y}$ que depende da tensão de controle $V_{c t r l}$. A tensão de controle é definida mediante um loop de retroalimentação durante a fase de aprendizado de maneira que o atraso introduzido pelo DE seja igual a:

$$
\Delta_{y}=T-K_{V T C} \times V_{y}
$$

sendo T um tempo constante menor que o período de amostragem. Dessa maneira o atraso total observado na saída do DE é:

$$
\Delta_{x}+\Delta_{y}=T+K_{V T C} \times\left(V_{x}-V_{y}\right)
$$

Finalmente uma porta XOR recebe como entradas a saída do DE e um pulso de referência que está atrasado em relação à borda de descida do reset em T. Portanto, para o caso $V_{x}>V_{y}$ a largura do pulso na saída da XOR é:

$$
\begin{aligned}
\Delta_{\phi} & =\Delta_{x}+\Delta_{y}-T \\
\Delta_{\phi} & =K_{V T C} \times\left(V_{x}-V_{y}\right)
\end{aligned}
$$

Uma análise similar para o caso em que $V_{x}>V_{y}$ prova que a largura do pulso de saída seria $K_{V T C} \times\left(V_{y}-V_{x}\right)$. Então, o circuito gera um pulso digital cuja duração é proporcional ao valor absoluto da diferença entre $V_{x}$ e $V_{y}$.

O pulso de saída da porta XOR pode ser aplicado a um integrador para gerar uma tensão proporcional ao valor absoluto da diferença, como se observa na figura 5.8.

Neste caso a tensão de saída é:

$$
V_{\text {diff }}=K_{V T C} \times \frac{I}{C} \times\left|V_{x}-V_{y}\right|
$$




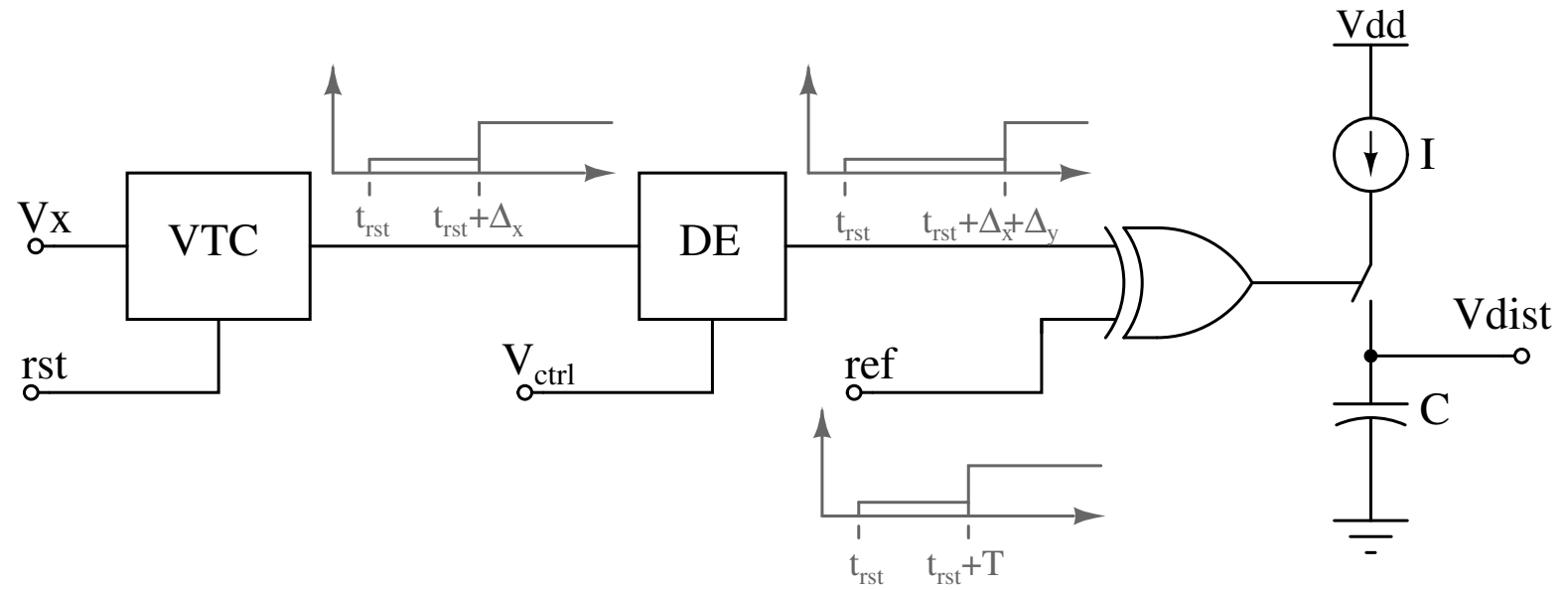

Figura 5.8: Circuito subtrator baseado em tempo com saída na forma de tensão.

\subsubsection{Programação do circuito subtrator}

Como mencionado anteriormente, antes de realizar a subtração o circuito subtrator deve ser programado ou configurado de maneira a aprender a amostra de referência $\left(V_{y}\right)$. Para esse fim é utilizado um loop de retroalimentação similar a um DLL (Delay Locked Loop). Esse loop é representado na figura 5.9.

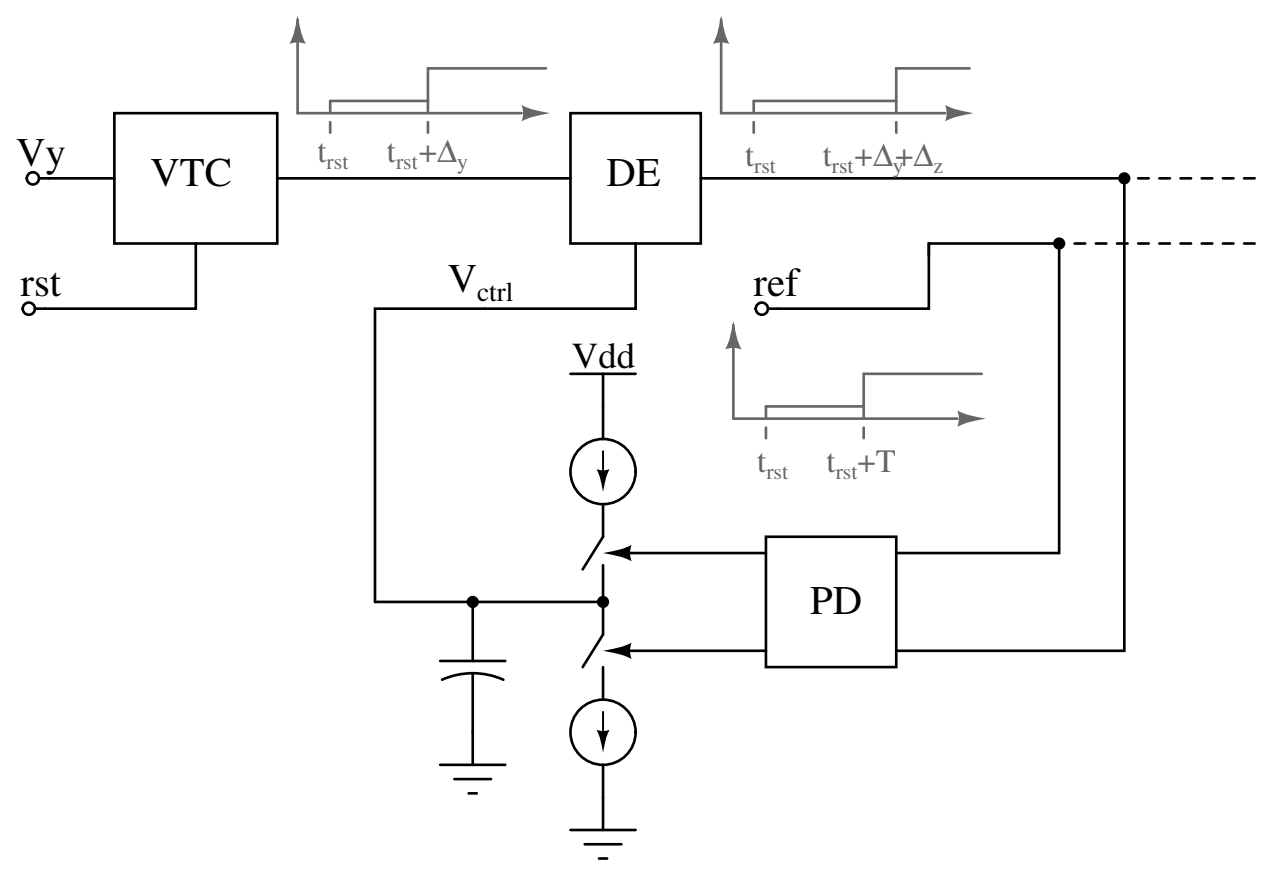

Figura 5.9: Programação do circuito subtrator para calcular diferenças em relação a $V_{y}$.

Durante a programação são aproveitados alguns elementos do circuito que se usam na operação normal. Estes elementos são o VTC e o DE. Além desses cir- 
cuitos são necessários adicionalmente um detector de fase e uma bomba de carga. $\mathrm{O}$ objetivo é estabelecer uma tensão de controle $\left(V_{c t r l}\right)$ em um capacitor que se encontra na saída da bomba de carga. Para isso a tensão que se deseja programar deve ser aplicada na entrada do VTC e o atraso da saída do DE é comparado com o mesmo pulso de referência usado na operação normal; ou seja um pulso atrasado em relação à borda de descida do reset em T. Para entender melhor o processo de programação do circuito subtrator é apropriado observar o diagrama de tempos da figura 5.10 que foi obtido mediante simulação elétrica.
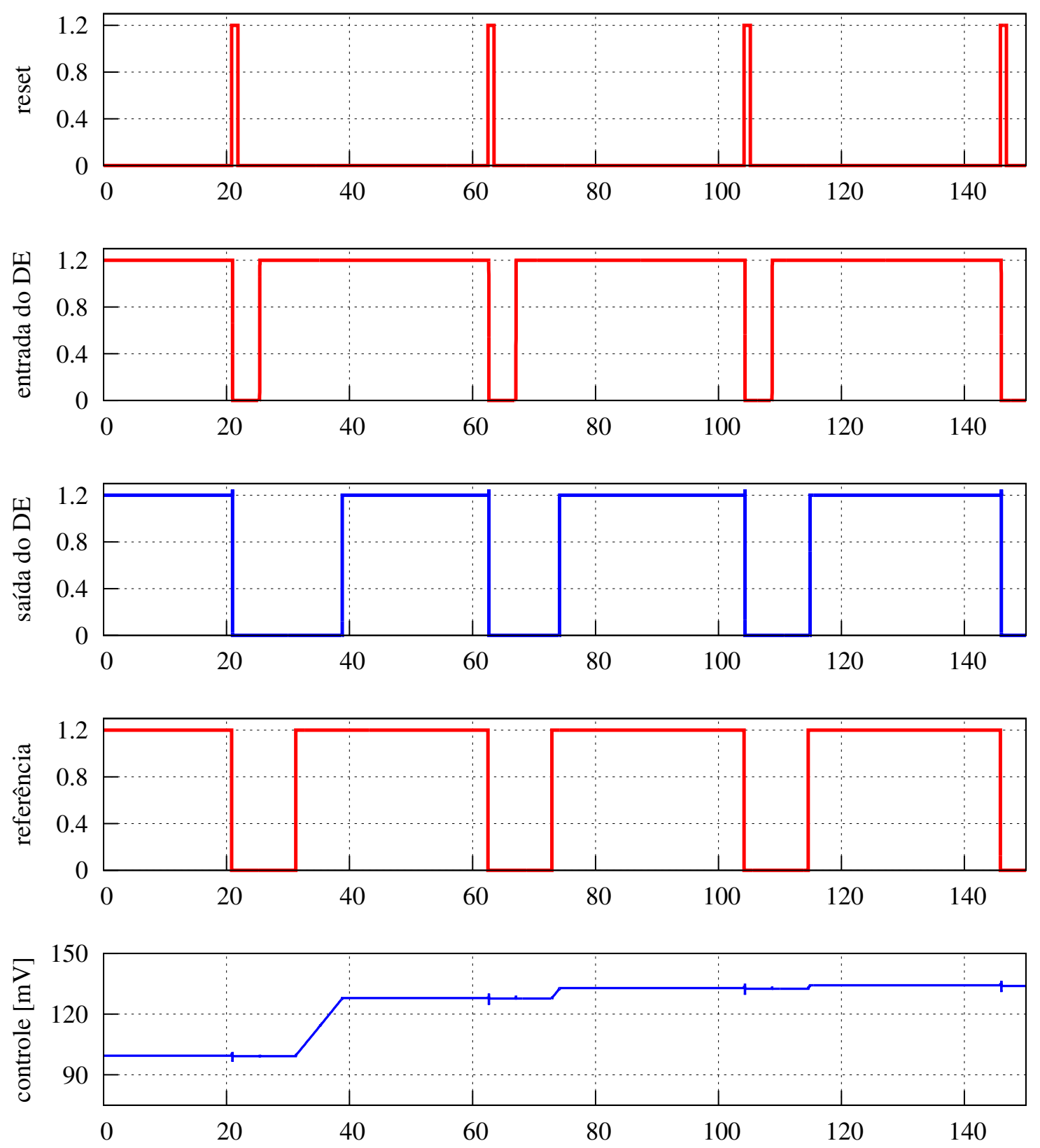

Figura 5.10: Diagramas de tempos obtidos mediante a simulação do loop de programação do circuito subtrator para calcular diferenças em relação a $V_{y}$. 
O objetivo do loop é obter uma tensão de controle de modo que o pulso de saída do DE esteja alinhado com o pulso de referência. Quando isso acontece se cumprem as seguintes relações:

$$
\begin{aligned}
T & =\Delta y+\Delta z \\
\Delta z & =T-\Delta y \\
\Delta z & =T-K_{V T C} \times V_{y}
\end{aligned}
$$

ou seja, o DE estará programado para introduzir o atraso necessário para realizar a subtração em relação a $V_{y}$ como estipulado na equação 5.12 .

O elemento de atraso (DE) utilizado neste sistema tem a estrutura mostrada na figura 5.11, a qual é similar ao do elemento de atraso apresentado na referência (NADEAU; PAIDIMARRI; CHANDRAKASAN, 2016).

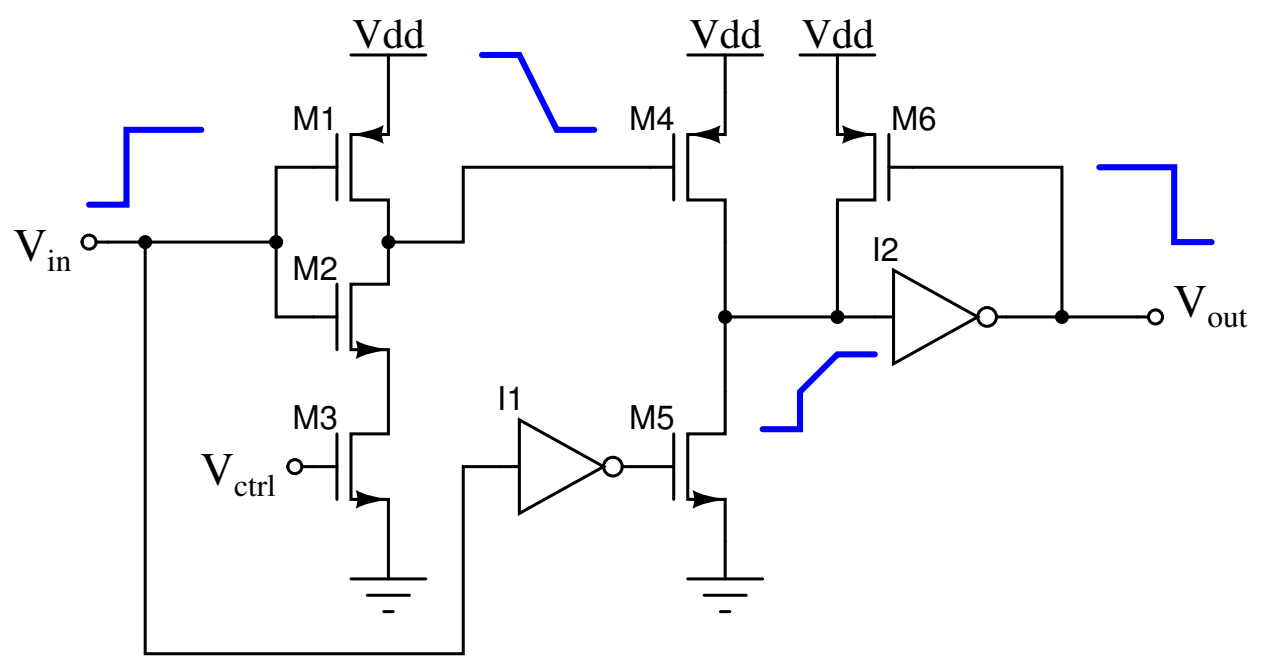

Figura 5.11: Esquema elétrico do elemento de atraso (DE da figura 5.9) utilizado neste trabalho.

Nessa figura, o pulso digital de entrada $\left(V_{\text {in }}\right)$ é aplicado a um inversor com corrente de descarga controlada formado pelos transistores M1, M2 e M3. Esse tipo de inversor é conhecido na literatura em inglês como Current Starved Inverter (DEJEMOUAI; SAWAN; SLAMANI, 1999), (PEKAU; YOUSIF; HASLETT, 2006). Em muitos dos elementos de atraso baseados no Current Starved Inverter, a saída deste inversor é aplicada a um inversor CMOS convencional. Dessa forma, o consumo de corrente do segundo inversor aumentaria devido ao fato que o sinal aplicado na entrada tem uma transição lenta. No elemento de atraso utilizado neste trabalho, mostrado na figura 5.11, o sinal não é aplicado diretamente a um inversor CMOS convencional, mas sim na porta do transistor 
M4. Quando o transistor M4 começa a conduzir, o transistor M5 é desligado, evitando assim a corrente de curto circuito e, portanto, reduzindo o consumo de potência. 


\subsection{Circuito que calcula a distância de Manhattan}

Empregando vários circuitos subtratores pode se implementar um circuito que calcula a distância de Manhattan. Uma implementação direta é representada na figura 5.12. Um único capacitor de saída integra as contribuições de cada subtrator de maneira que ao final da operação a tensão do capacitor é a soma das diferenças calculadas pelos subtratores.

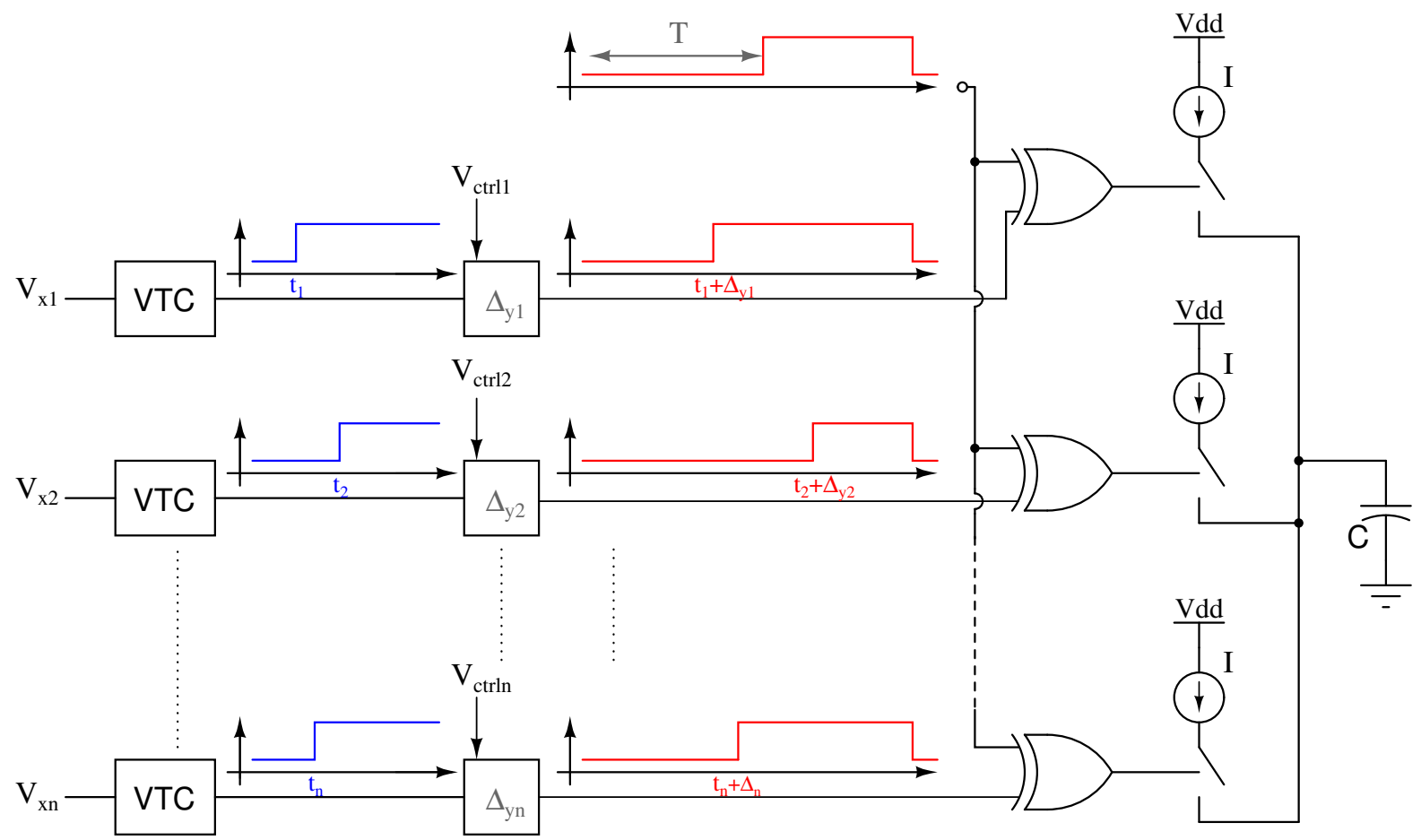

Figura 5.12: Circuito que calcula a distância de Manhattan baseado no uso de uma fonte de corrente para cada canal. As correntes são integradas no capacitor de saída gerando uma tensão proporcional à distância de Manhattan. 
Um problema associado à essa implementação é sua sensibilidade ao mismatch entre as fontes de corrente. Isso pode ser resolvido usando apenas uma fonte de corrente como mostrado na figura 5.13. Neste caso os subtratores não podem operar simultaneamente. Dada essa característica sequencial da operação do circuito é possível compartilhar um único VTC entre todos os subtratores.

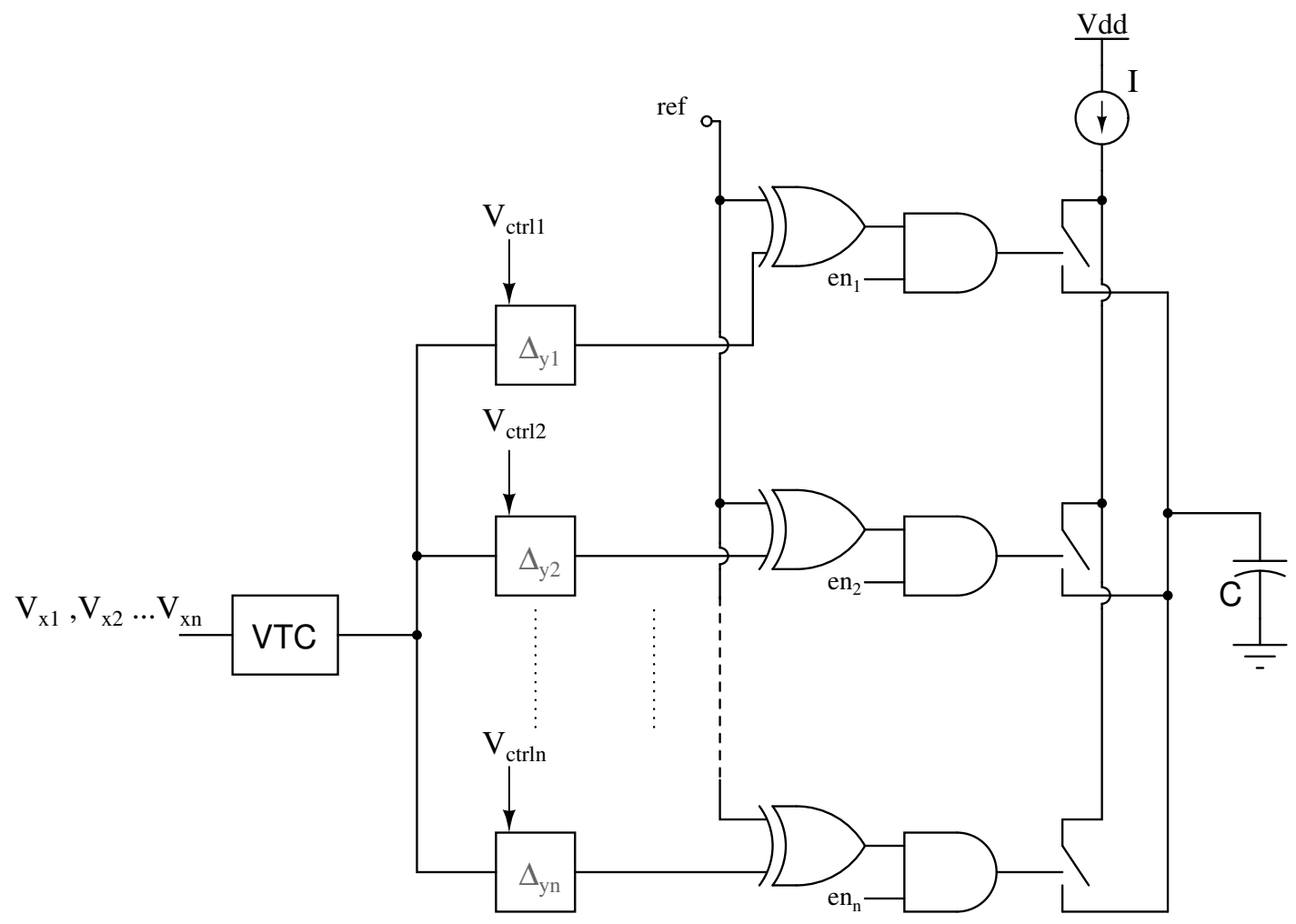

Figura 5.13: Circuito que calcula a distância de Manhattan com uso compartilhado de VTC e de integrador de saída

Nessa implementação as amostras devem ser aplicadas de maneira sequencial. A tensão do capacitor de saída é incrementada cada vez que uma operação de subtração é finalizada. Após o processamento de todas as amostras a tensão de saída será proporcional à distância deManhattan entre o padrão definido pelas amostras de entrada e o padrão definido pelas tensões configuradas nos circuitos DE.

$$
V_{d i s t}=K_{V T C} \times \frac{I}{C} \times \sum_{i=1}^{n}\left|V_{x i}-V_{y i}\right|
$$




\subsection{Simulação do sistema de classificação de impul- sos elétricos desenvolvido nesta tese}

Para avaliar o desempenho do sistema de classificação desenvolvido realizaram simulações elétricas aplicando um sinal construído a partir de spikes reais. O sinal contém spikes de três neurônios e ruído. O valor rms de ruído é igual ao $20 \%$ da amplitude máxima dos spikes. O sinal contém um total de 2586 spikes e com base no $20 \%$ dos spikes de cada neurônio construíram-se os protótipos que os representam. Na figura 5.14 apresentam-se os três protótipos:
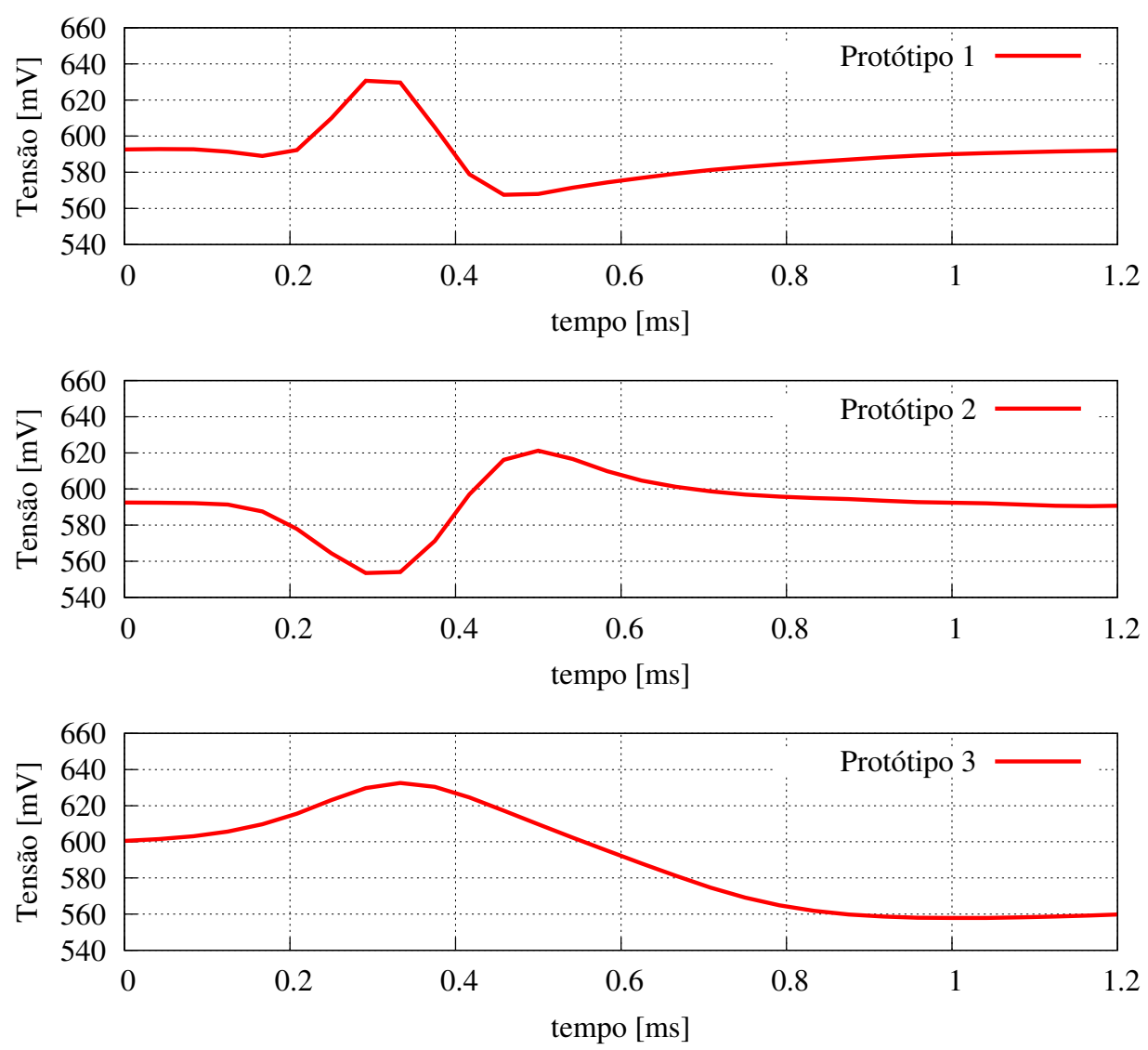

Figura 5.14: Spikes de três neurônios diferentes, presentes no sinal utilizado na simulação do sistema de classificação.

Cada spike é representado mediante 30 amostras adquiridas a uma frequência de $24 \mathrm{KS} / \mathrm{s}$. As amostras dos protótipos foram aplicadas ao sistema na fase de treinamento com o objetivo de configurar os subtratores baseados em tempo tal como foi explicado na subseção 5.3.2. Cada protótipo é processado por um circuito que calcula a distância de Manhattan.

Após a fase de configuração dos subtratores foram aplicados 1996 spikes ao sis- 
tema. Cada spike é processado pelos três circuitos que calculam a distância de Manhattan. Cada um desses circuitos calcula a distância entre o spike de entrada e o protótipo que foi configurado.

Na figura 5.15 observa-se o resultado da simulação em que foi aplicado um spike da classe 1 (mesma classe do protótipo 1). Na parte superior dessa figura estão três gráficos que permitem comparar visualmente ao spike aplicado com os três protótipos. Os resultados do cálculo da distância entre esse spike e os três protótipos estão representados nos três gráficos da parte inferior.
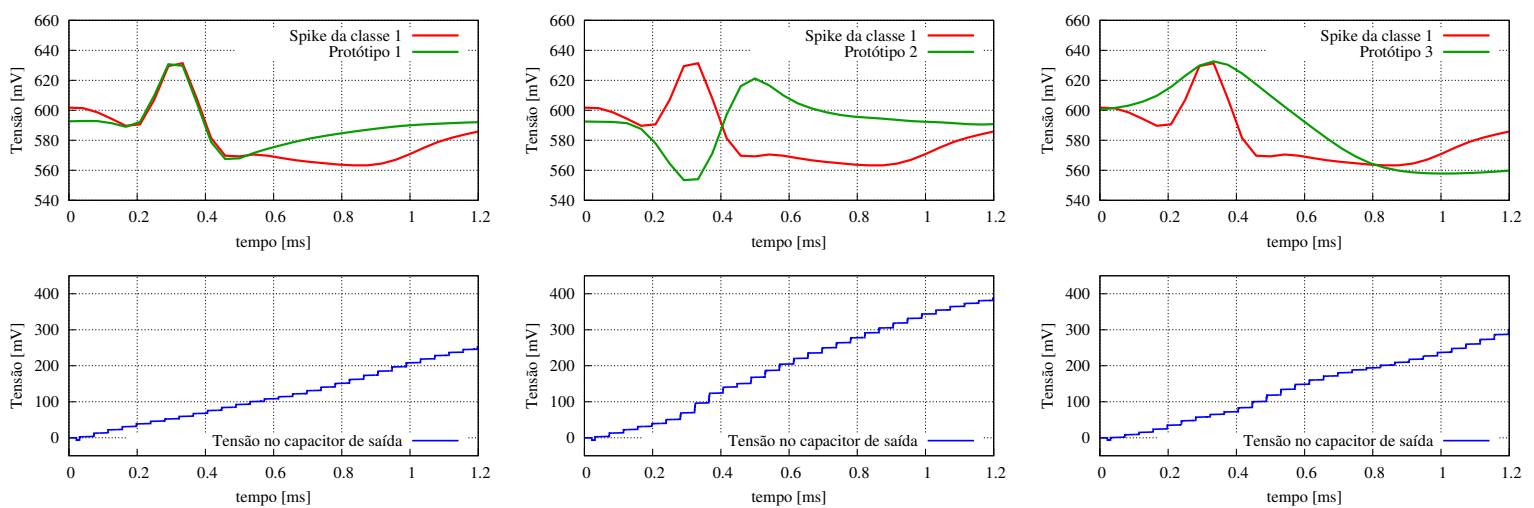

Figura 5.15: Resultados de simulação da aplicação de um spike da classe 1 ao sistema.

Observando as tensões no capacitor de de saída do circuito (três gráficos da parte inferior) nota-se que a menor distância corresponde à classe 1. A diferença entre a menor das tensões e a segunda menor tensão é aproximadamente $50 \mathrm{mV}$. Essa diferença de tensões é facilmente detectada por um comparador de tensão. O fato que a menor tensão corresponda à classe 1 significa que o sistema classificou corretamente ao spike de entrada. 
Na figura 5.16 observa-se o resultado para o caso da aplicação de um spike da classe 2.
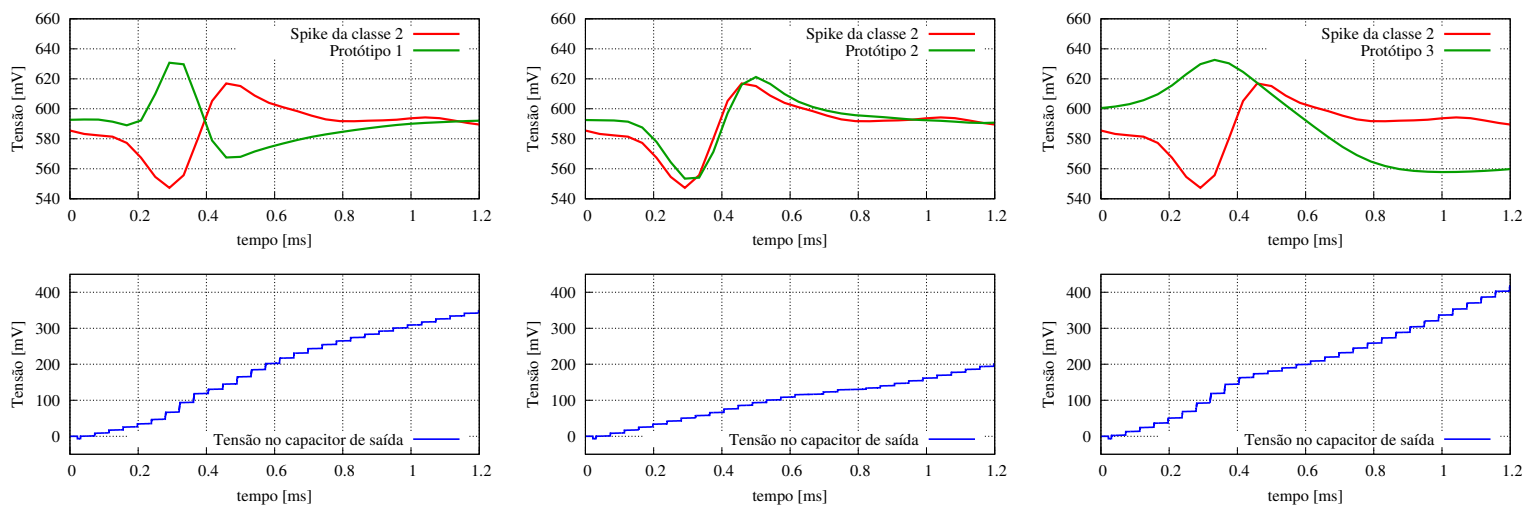

Figura 5.16: Resultados de simulação da aplicação de um spike da classe 2 ao sistema.

De novo, o sistema gera tensão mínima na comparação com o protótipo correto (neste caso, o protótipo 2).

Finalmente, na figura 5.17 observa-se o resultado para o caso da aplicação de um spike da classe 3.
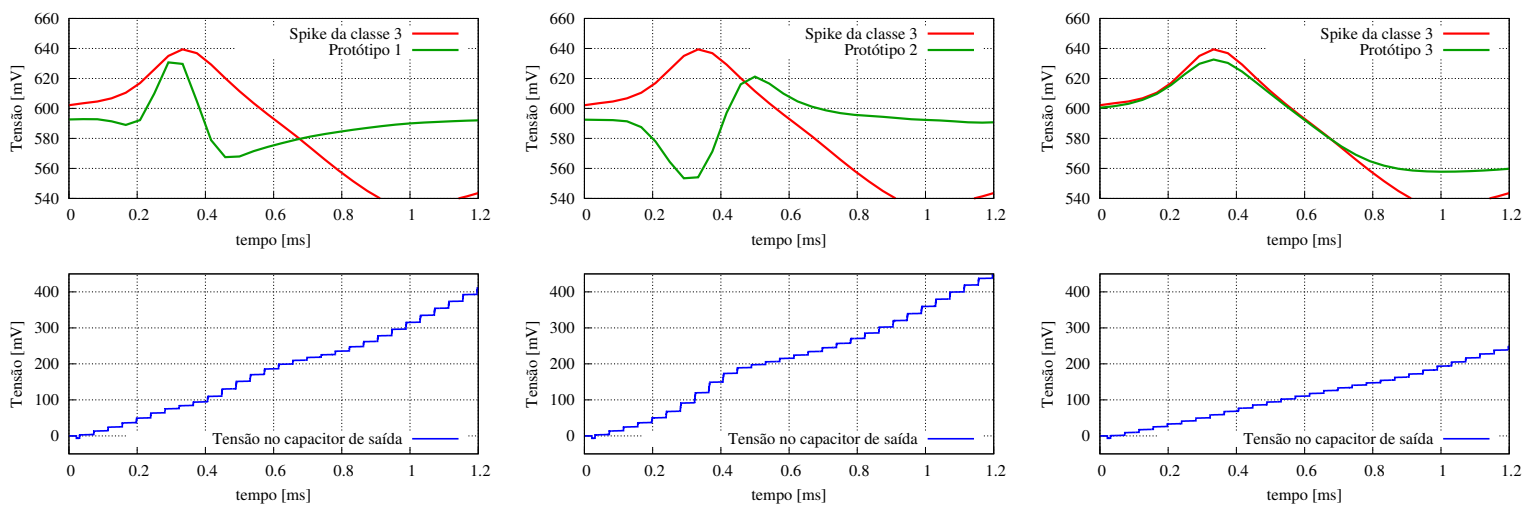

Figura 5.17: Resultados de simulação da aplicação de um spike da classe 3 ao sistema.

Observa-se que o sistema gera tensão mínima na comparação com o protótipo correto (neste caso, o protótipo 3).

Quando um spike de entrada é comparado, através do cálculo da distância de Manhattan, com os três protótipos, a menor das distâncias define o resultado da classificação. A menor das três distâncias indica a qual das três classes pertence o spike de entrada. Um erro do sistema de classificação consiste em indicar uma 
classe diferente à real. O comparador de saída, que identifica a menor das distâncias pode indicar um resultado incorreto se as tensões de saída são próximas. Por exemplo se a menor das distâncias é $300 \mathrm{mV}$ e a segunda menor distância é $301 \mathrm{mV}$ é muito provável que o comparador de saída indique uma classe errada devido ao offset. Embora seja possível aplicar técnicas de auto-zero para reduzir o offset é desejável que as diferenças entre distâncias sejam maiores ao valores típicos de offset. Quando os formatos dos protótipos sejam bem similares será praticamente impossível evitar que as distâncias sejam muito próximas e neste caso acontecerá um erro na classificação.

A precisão da classificação definida como a relação entre o número de spikes corretamente classificados e o número total de spikes depende da mínima diferença de tensão que o comparador de saída consiga resolver. Para avaliar essa precisão se aplicaram 200 spikes do banco de dados escolhido. Na figura 5.18 se observa a precisão da classificação em função da mínima tensão que pode ser reconhecida pelo comparador de saída.

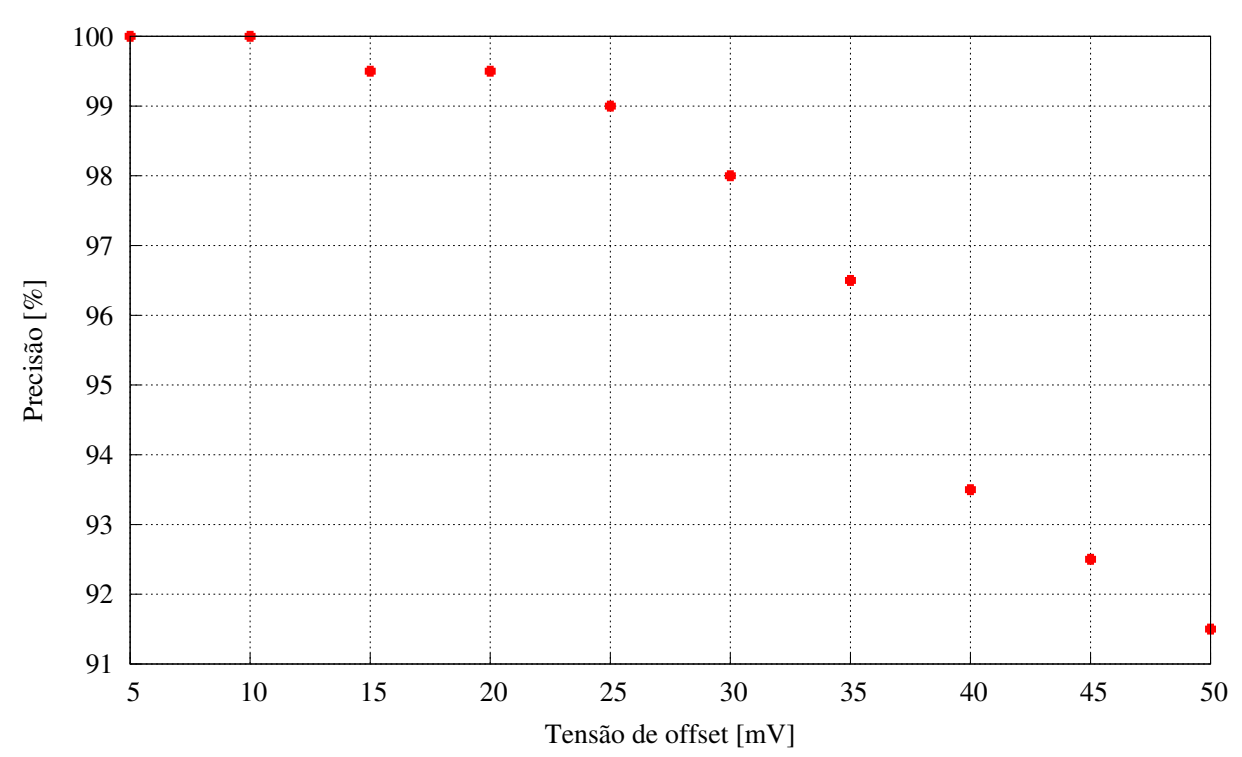

Figura 5.18: Precisão da classificação em função da mínima tensão que o comparador de saída pode reconhecer

Como foi mencionado, o sistema apresentado pode operar diretamente com amostras analógicas ou com amostras digitais. Os resultados apresentados correspondem ao caso de operação com amostras analógicas em que é necessário o uso de um conversor de tensão a tempo (VTC: Voltage-to-time converter) o qual consome $1.5 \mu \mathrm{W}$. Cada circuito que calcula a distância de Manhattan consome $1 \mu \mathrm{W}$ e o comparador de saída consome também $1 \mu \mathrm{W}$. Esse valores incluem consumos estáticos e dinâmicos. Com isso o consumo total por canal é de $4.5 \mu \mathrm{W}$ e por exemplo para o caso de 64 
canais o consumo total seria $298 \mu \mathrm{W}$. Para poder comparar este consumo com os de outros trabalhos, como por exemplo o de (KARKARE; GIBSON; MARKOVIC, 2013) que é 75 $\mu \mathrm{W}$ (sem considerar o consumo necessário para a conversão de amostras ao domínio digital), é importante lembrar que o sistema aqui apresentado opera com uma tensão de alimentação de $1.2 \mathrm{~V}$, e que por tratar-se de uma estratégia baseada em tempo, o consumo seria proporcionalmente menor se a tensão fosse menor. Por exemplo, para uma tensão de alimentação de 0.5V (como na referência (KARKARE; GIBSON; MARKOVIC, 2013)) estima-se, através de um cálculo conservador, que o consumo do sistema desenvolvido nesta tese seria próximo de $125 \mu \mathrm{W}$. Também é importante considerar que a operação direta com amostras analógicas, adotada nesta tese, dispensa o uso do conversor analógico digital, o qual é substituído pelo VTC. Sem considerar a conversão, o consumo para 64 canais com tensão de alimentação de $0.5 \mathrm{~V}$ seria aproximadamente $93 \mu \mathrm{W}$. 


\section{Conclusões e perspectivas}

A seguir são apresentadas as conclusões da pesquisa de doutorado relatada neste texto. As conclusões estão organizadas em três grupos, o primeiro trata da pesquisa sobre interfaces neurais implantáveis e os processamentos realizados nelas, o segundo sobre o projeto e simulação do sistema de detecção de spikes e o último sobre o sistema de classificação de spikes.

\subsection{Sobre o estado da arte e o rumo da pesquisa em interfaces neurais implantáveis}

As conclusões apresentadas a seguir, foram obtidas com base na pesquisa em artigos de congressos e periódicos.

- Na pesquisa em neurociência é relevante dispor de equipamentos que realizem o registro de sinais intracorticais com a possibilidade de identificar instantes de disparos de neurônios isolados.

- Na área de estudo de neuropróteses, é importante conhecer os instantes de disparos de neurônios isolados porque mesmo estando próximos, dois neurônios podem codificar informações completamente diferentes.

- Com a tecnologia atual, cada eletrodo implantado recebe sinais de vários neurônios simultaneamente.

- A tendência atual na área de neuropróteses é o aumento de número de eletrodos que realizam registro de sinais extracelulares. Essa tendência impõe desafios relacionados com o consumo de potência e largura de banda da informação a ser transmitida. 
- Existe interesse em estudar alternativas para a implementação em hardware do processo de spike detection e spike sorting, visando a redução do consumo de potência em interfaces neurais implantáveis.

\subsection{Sobre o sistema de detecção de impulsos elétricos neurais desenvolvido nesta tese}

- Foi realizado um estudo para determinar se é apropriado utilizar o valor absoluto de um sinal neural como entrada do operador NEO. Mediante a simulação dessa alternativa em sinais do banco de dados Waveclus com 8 diferentes níveis de ruído concluiu-se que é equivalente ao caso da aplicação direta do NEO ao sinal original. Essa conclusão foi obtida tanto através de comparação visual das ondas resultantes quanto mediante a comparação de parâmetros de detecção tpr (true positive rate) e fpr (false positive rate). Este tipo de análise pode contribuir, no futuro, ao desenvolvimento de novas estratégias de detecção que resultem em um hardware mais compacto. Notou-se, no estudo do estado da arte, que alguns pesquisadores optam por fazer pequenas modificações a algoritmos já bem estabelecidos e testados em software com o objetivo de obter soluções mais econômicas em termos de área e consumo de potência.

- Foi desenvolvido um circuito elevador ao quadrado baseado em três transistores PMOS que opera em modo corrente e que pode ser usado no sistema que calcula o NEO. Embora apresente uma dependência com parâmetros de processo e temperatura, essa dependência pode ser eliminada a nível do sistema de detecção de spikes, através de seleção apropriada do limiar de comparação. O circuito elevador ao quadrado e esta estratégia para eliminar a dependência de parâmetros de processo e temperatura têm o potencial de aproveitamento em outras implementações de detectores de spikes ou em outros tipos de processamentos não lineares. É interessante acrescentar que a otimização do conjunto das tarefas de conversão de tensão a corrente e de elevação ao quadrado pode ser útil para um grande conjunto de aplicações futuras. Algo nesse sentido foi ensaiado e publicado em (SALDAÑA-PUMARICA; DEL-MORAL-HERNANDEZ, 2016a).

- Para a simulação e caracterização do circuito elevador ao quadrado foi elaborado um programa em linguagem OCEAN (Open Command Environment for Analysis) compatível com as ferramentas da simulação de companhia CADENCE. 
Esse programa permite avaliar o quanto se aproxima a curva característica de saída do circuito em relação a uma função quadrática. Para isso empregou-se o coeficiente de correlação linear entre ambas as curvas. O programa permite realizar iterações mudando parâmetros de projeto, realizando simulações e observando tal coeficiente. Dessa maneira foi possível explorar o espaço de projeto do circuito elevador ao quadrado. Este tipo de programas contribui a uma tendência atual em usar, no contexto de projeto de circuito integrados, algoritmos de otimização e exploração do espaço das variáveis de projeto. Esse tipo de abordagem torna-se cada vez mais comum num cenário em que os modelos matemáticos dos dispositivos são cada vez mais complexos.

- Foi desenvolvida uma estratégia de implementação do NEO mediante o uso de 4 instâncias do circuito elevador ao quadrado mencionado no item anterior. 0 circuito obtido foi avaliado como estágio de pré-processamento na detecção de spikes e consumo, obtido mediante simulação, foi de 300 pJ para cada spike processado (30 amostras do sinal de entrada) (ver 4). A capacidade do circuito de salientar a presença de spikes foi comprovada mediante a simulação com sinais do banco de dados Waveclus (QUIROGA; NADASDY; BEN-SHAUL, 2004). Como trabalho futuro, propõe-se construir curvas de desempenho em função da área estimada considerando o efeito do descasamento de parâmetros entre dispositivos.

\subsection{Sobre o sistema de classificação de impulsos elétricos neurais desenvolvido nesta tese}

- Foi proposto um método para o cômputo da distância de Manhattan baseado na representação de sinais mediante atrasos entre pulsos digitais. O processamento com esse tipo de representação é conhecido na literatura como processamento em modo tempo (time-mode processing), processamento baseado em tempo (time-based processing) ou processamento no domínio do tempo (timedomain processing). A diminuição da máxima tensão de alimentação dos circuitos integrados é uma tendência conduzida pela necessidade de otimizar o desempenho dos circuitos digitais. Porém, para o projeto de circuitos analógicos essa diminuição da tensão de alimentação traz consequências adversas. Considerando os tipos clássicos de representação de grandezas analógicas (tensão, 
corrente e carga), as melhoras no processos de fabricação não se traduzem em melhoras em termos de desempenho dos circuitos analógicos. Nesse cenário, uma alternativa estudada atualmente é a representação baseada em tempo. Destaca-se, então, que o sistema de classificação de spikes projetado nesta tese contribui a esse aspecto do estado-da-arte.

- Foi projetado um circuito que implementa o método mencionado no item anterior. O circuito gera uma tensão de saída proporcional a distância de Manhattan entre dois conjuntos de grandezas analógicas ou digitais. A escolha entre a opção analógica ou digital deve ser acompanhada de um circuito de codificação apropriado. Neste trabalho apresentou-se o projeto do conversor de tensão a tempo (VTC de Voltage-to-Time Converter), necessário para a operação do sistema com grandezas analógicas. Como trabalho futuro recomenda-se implementar o conversor DTC (Digital-to-Time Converter) que permitirá explorar as possibilidades do sistema de operar com amostras digitais.

- Foi projetado um circuito que implementa a conversão de tensões analógicas de entrada em atrasos entre pulsos digitais de maneira tal que os atrasos são proporcionais às tensões de entrada. O consumo médio desse circuito é menor a $900 \mathrm{nW}$ como verificado na seção 5.2 .

- Foi projetado um sistema de classificação de spikes conformado por vários circuitos que calculam a distância de Manhattan. Como mostrado nas figuras 5.15, 5.16 e 5.17, que foram obtidas mediante simulação, o sistema consegue indicar a menor distância entre spike de entrada e templates para aquele template que representa a classe à qual o spike pertence. Os erros na classificação se devem, principalmente, à resolução do comparador de saída. Se a diferença entre a mínima distância e a segunda menor distância é menor que o offset do comparador de saída então pode acontecer um erro na classificação. Como mostrado na figura 5.18 o sistema consegue uma precisão de classificação maior a 99\% considerando um offset de $15 \mathrm{mV}$.

- Foi desenvolvido um programa na linguagem (OCEAN) que permite obter, através de um algoritmo de busca binária, as tensões de configuração dos elementos de atraso que definem o template que se deseja programar no sistema de classificação desenvolvido. Este programa facilitará, num projeto futuro, a avaliação do sistema operando com amostras digitais. 
- Foi desenvolvido um programa na linguagem (OCEAN) que permite aplicar de maneira sequencial as amostras de vários spikes ao circuito classificador e obter os resultados da classificação. Dessa maneira foram realizadas de maneira automática a simulação do sistema com mais de 400 spikes de entrada. Esse mesmo programa pode ser utilizado, no futuro, para avaliar o desempenho da operação sistema com amostras digitalizadas.

\subsection{Propostas para trabalhos futuros}

- Um ponto que deve ser estudado é a importância da taxa de acerto de detecção para a decodificação de comandos motores. Uma primeira atividade, além da pesquisa na literatura existente, é a realização de ensaios computacionais onde seja possível variar a taxa de acerto de detecção e observar o efeito no acerto da decodificação de comandos motores. Para isso seria necessário dispor de um banco de dados de sinais neurais coletados em algum experimento de BMI (Brain Machine Interface).

- No sistema de detecção considerou-se que o limiar pode ser programado. Porém, existem na literatura algumas tentativas do cálculo automático do limiar a partir da análise do sinal (HARRISON; CHARLES, 2003). Essas análises poderiam estender-se ao caso do sinal do saída do circuito NEO apresentado nesta tese.

- A utilização das estratégias de processamento analógico apresentadas neste trabalho também poderiam ser estendidas ao caso de sinais de Eletrocorticograma (ECoG), que se trata de um método menos invasivo (MULLER et al., 2015). Essa extensão pode resultar em trabalhos futuros de mestrado ou doutorado.

- Para o sistema de classificação, adotou-se a distância de Manhattam como parâmetro para comparar formatos de impulsos neurais. A representação baseada em tempo permitiu uma implementação simples para o cálculo dessa distância. Outras distâncias podem ser avaliadas em trabalhos futuros considerando dois aspectos: o primeiro é relacionado à sua influencia nos resultados de classificação e o segundo é a simplicidade da sua implementação em hardware. 


\section{Apêndice A - Programas em Matlab}

Este apêndice contém todos os programas em matlab utilizados nas simulações realizadas como parte deste trabalho de doutorado. Incluem-se programas relacionados à detecção de impulsos elétricos neurais, classificação de impulsos elétricos neurais e à análises de desempenho de alguns circuitos desenvolvidos neste trabalho. Desta maneira, facilita-se ao pesquisador interessado a reprodução dos resultados obtidos nesta tese (através de uma operação "colar e pegar").

\section{A.1 Extração e filtrado de sinais do banco de dados Waveclus}

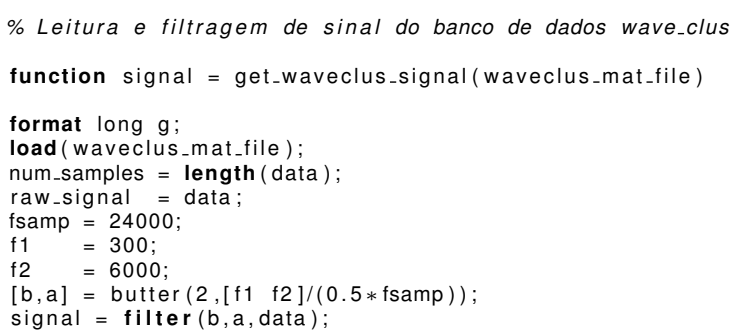

\section{A.2 Extração de instantes de ocorrência de spikes em um sinal do banco de dados Waveclus}

function times $=$ get_waveclus_times ( waveclus_mat_file)

format long $\mathrm{g}$;

load ( waveclus_mat_file);

times = cell2mat (spike_times $)$;

\section{A.3 Geração de tabela de resultados do NEO imple- mentado em matlab e aplicado a sinais do banco de dados Waveclus}




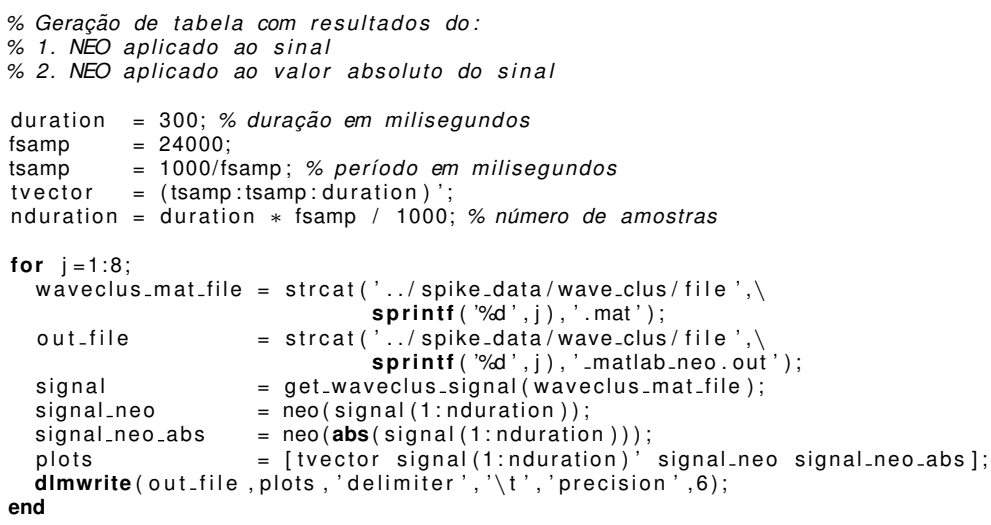

end

\section{A.4 Extração e filtrado de sinais do banco de dados Osort}

function signal = get_osort_signal (osort_sim_number, osort_noise_level)

format long $g$;

osort_mat_file $=\operatorname{strcat}($ ' .../spike_data/osort/sim', sprintf( '\%d', osort_sim_number), 1

load (osort_mat_file):

raw_signal = spiketrains (osort_noise_level)

fsamp = 25000;

signal $=$ cell2mat $($ raw_signal $)$

\section{A.5 Extração de instantes de ocorrência de spikes em um sinal do banco de dados Osort}

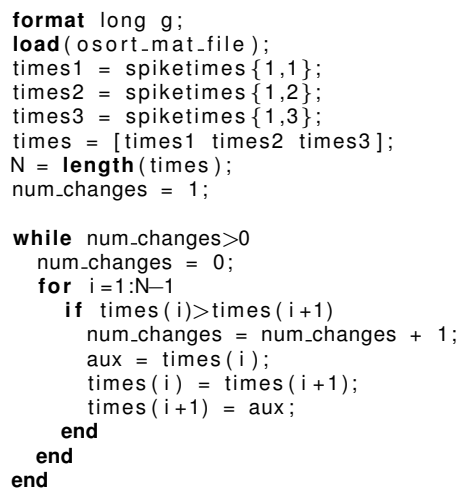

\section{A.6 Construção de curva ROC}

function roc=roc3 (signal, reference_times, fsamp, trefrac, kinf, ksup)

format long g;

num_samples = length ( signal);

$\%$ Number of samples of refractory period (ex 48)

nrefrac $=$ trefrac $*$ fsamp

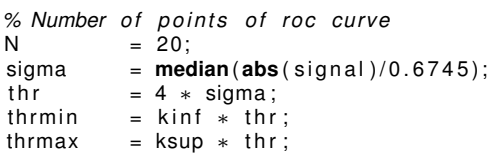


thrstep $=(\text { thrmax } / \text { thrmin })^{\wedge}(1 /(\mathrm{N}-1))$;

roc $=\operatorname{zeros}(N, 3)$;

for $i=1: N$

thri $=$ thrmin + thrmax - thrmax / thrstep ${ }^{\wedge}(i-1)$

detected times $=$ threshold2 (signal, thri, nrefrac) ;

[ntdi tpri nfdi fpri] = evaluate_detector (detected_times,

reference_times, num_samples, nrefrac ,fsamp);

end

pri ]; 


\section{Apêndice B - Programas em OCEAN}

As ferramentas de projeto e simulação da companhia CADENCE utilizadas no desenvolvimento desta tese podem ser programas através de uma linguagem chamada OCEAN (Open Command Environment for Analysis). A seguir apresentam-se os programas desenvolvidos nessa linguagem que foram empregados em várias das simulações realizadas neste trabalho.

\section{B.1 Programa para a explorar o espaço de projeto do circuito elevador ao quadrado}

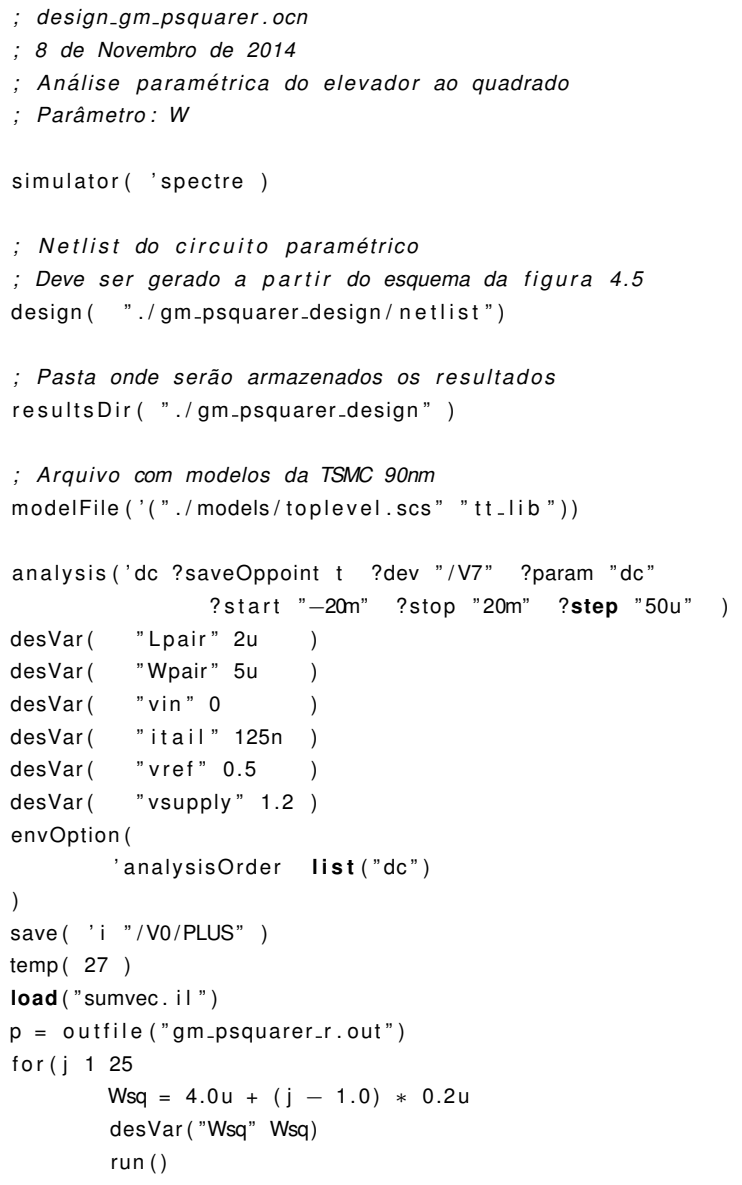




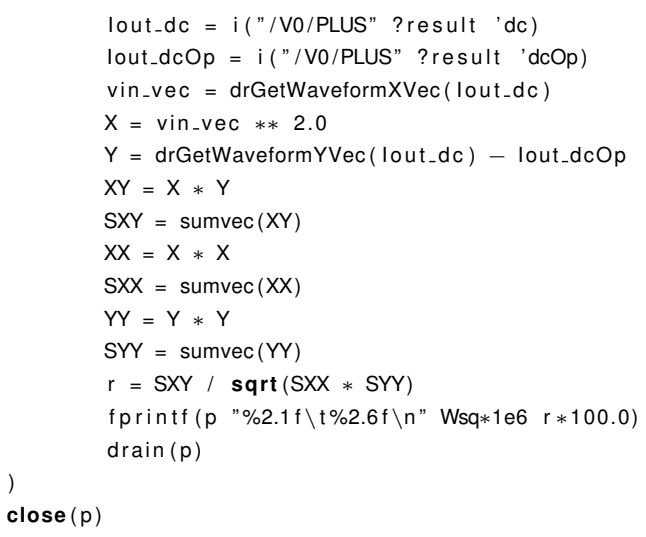

\section{B.2 Programa para o a simulação do circuito NEO em regimen DC}

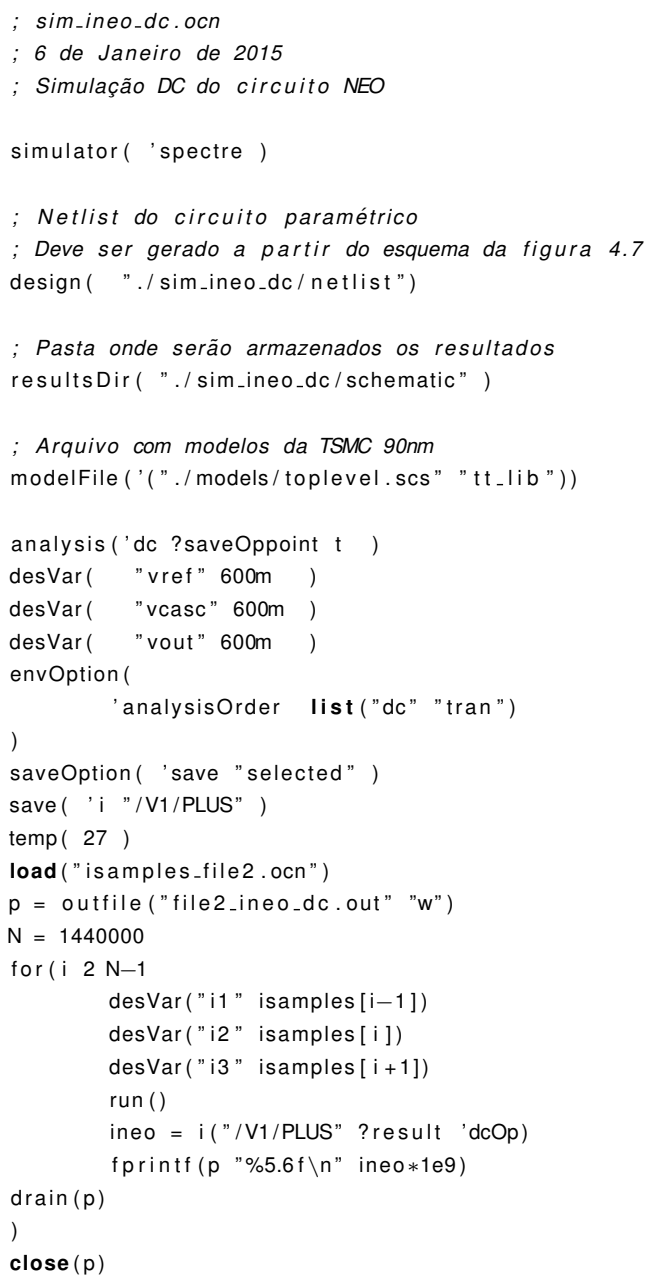

\section{B.3 Programa para a obter a tensão de calibração dos elementos de atraso}




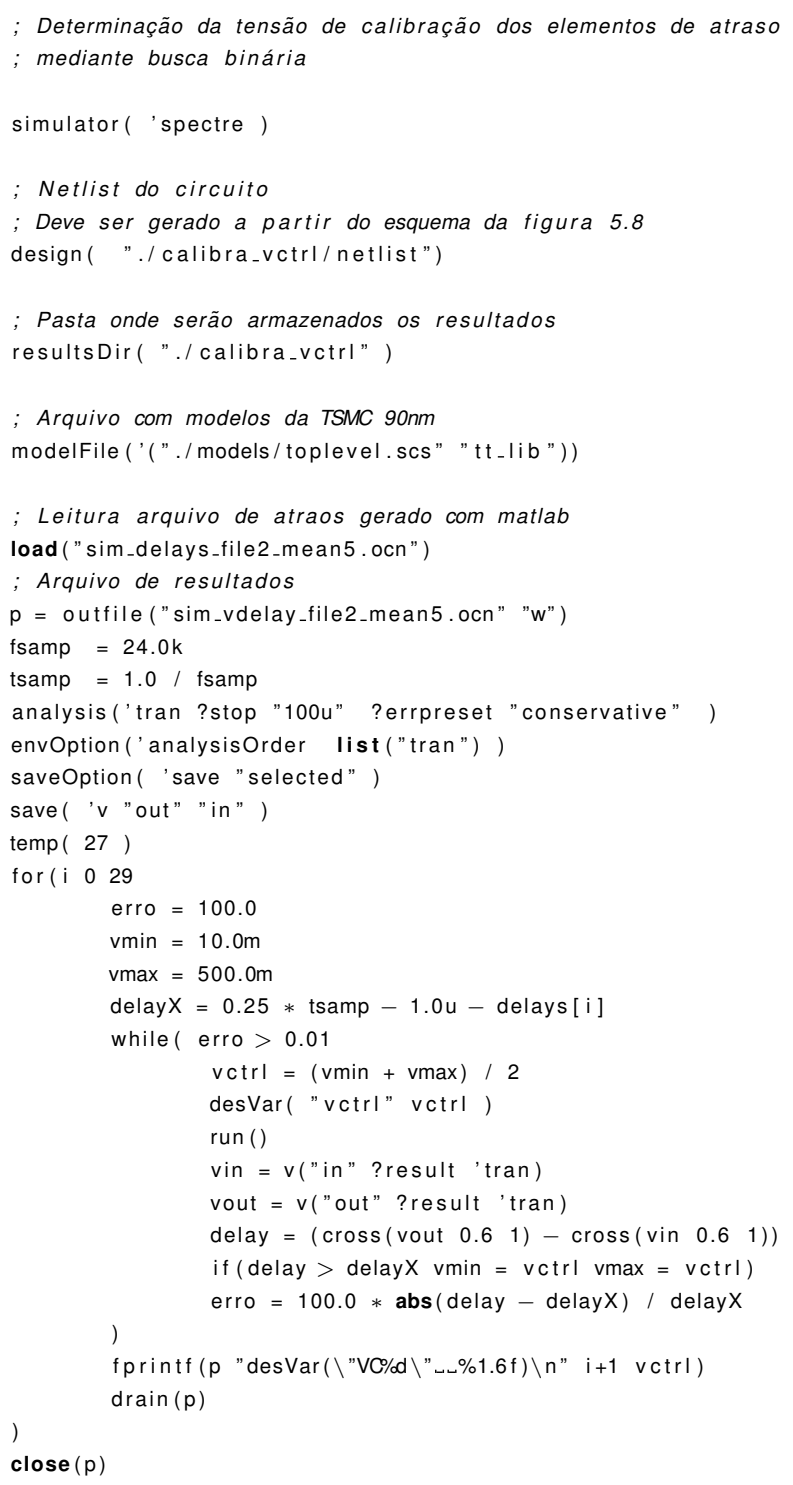

\section{B.4 Programa para obter os parâmetros de deteção com base nos resultados da simulação do detec- tor de spikes}

procedure ( evaluate_detector (detected_times,

reference_times num_samples spike_duration sampling_frequency)

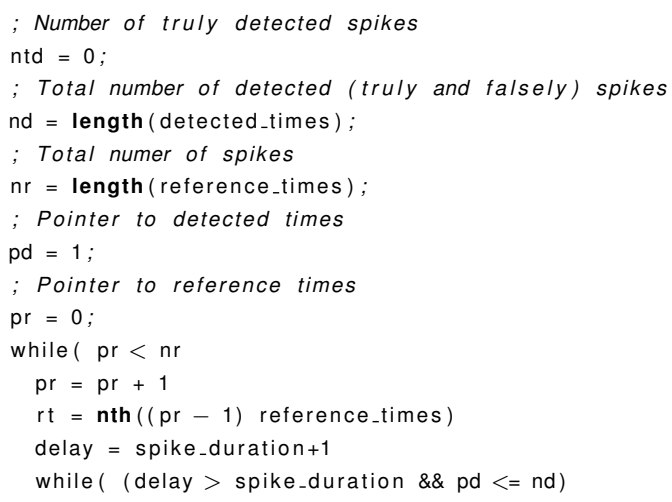




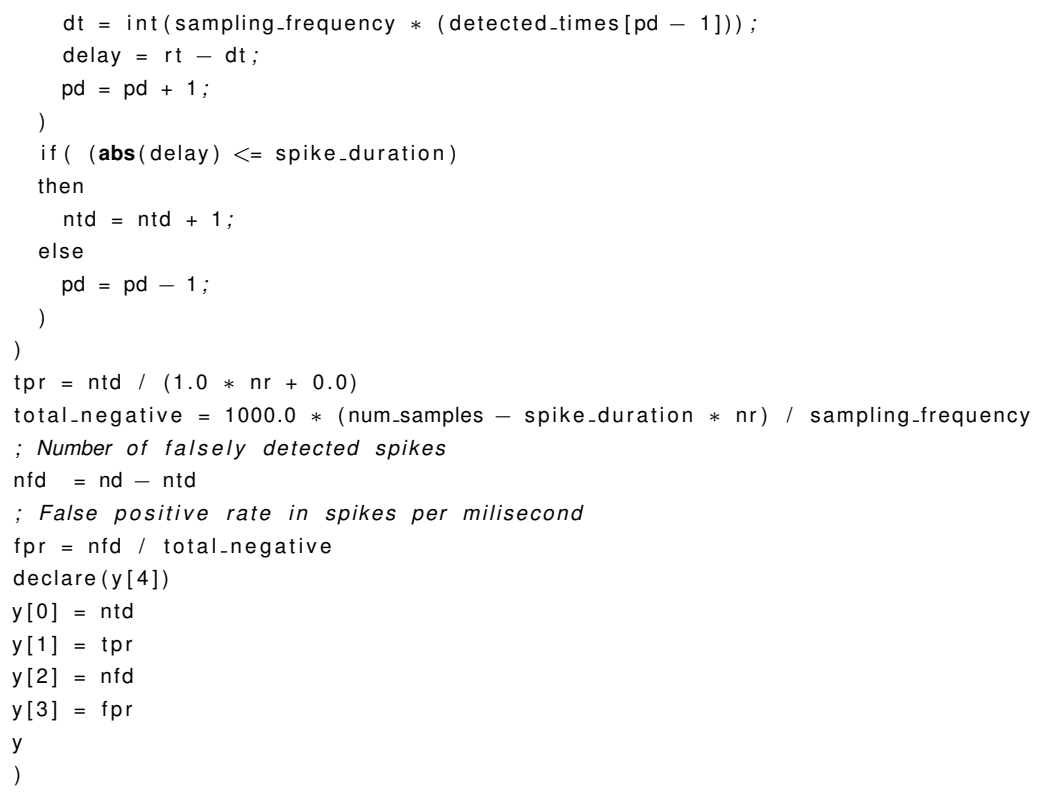

\section{B.5 Programa para a obter a curva ROC}

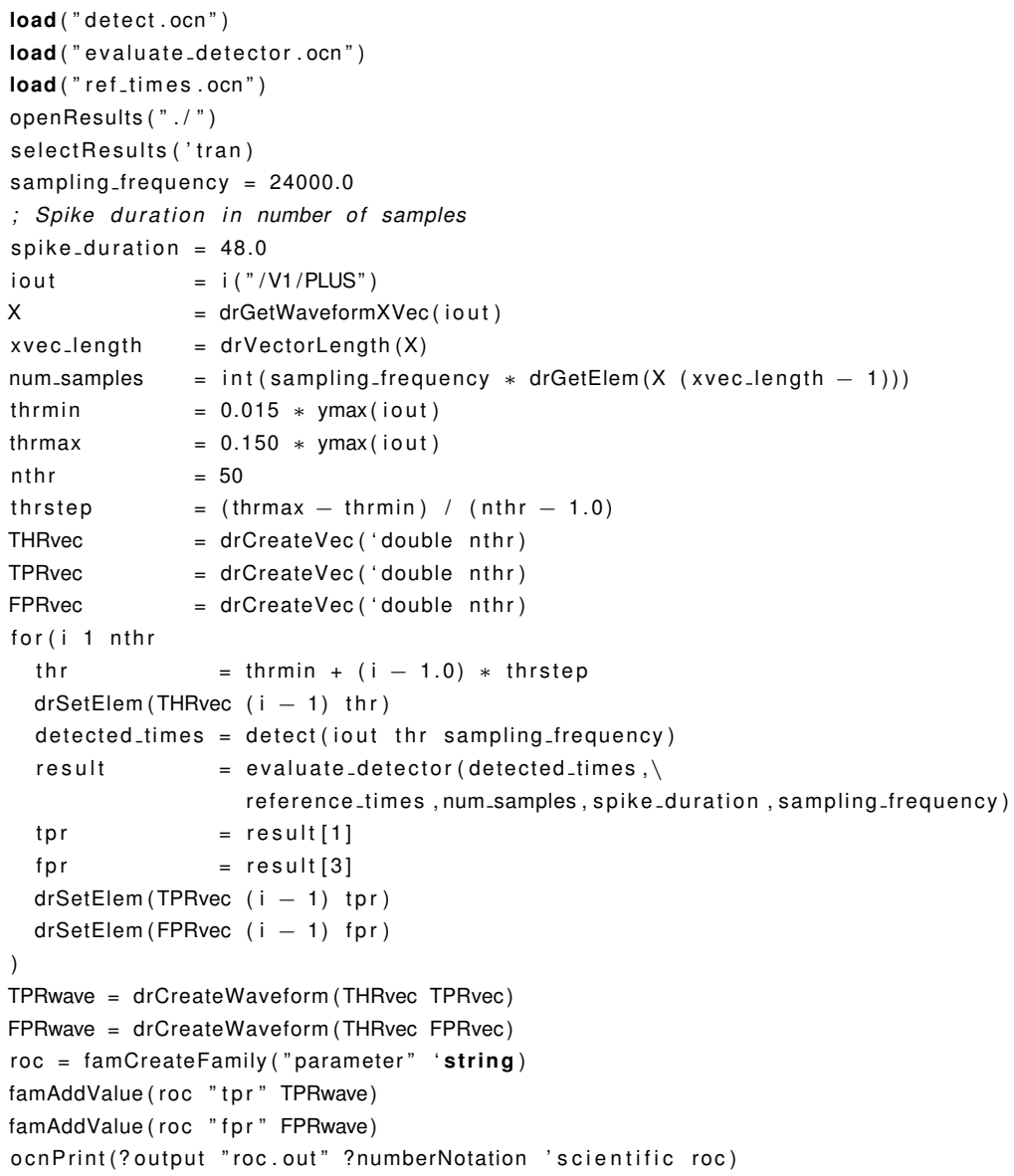




\section{B.6 Programa para simulação do cômputo da distância entre dos vetores de grandezas analógicas}

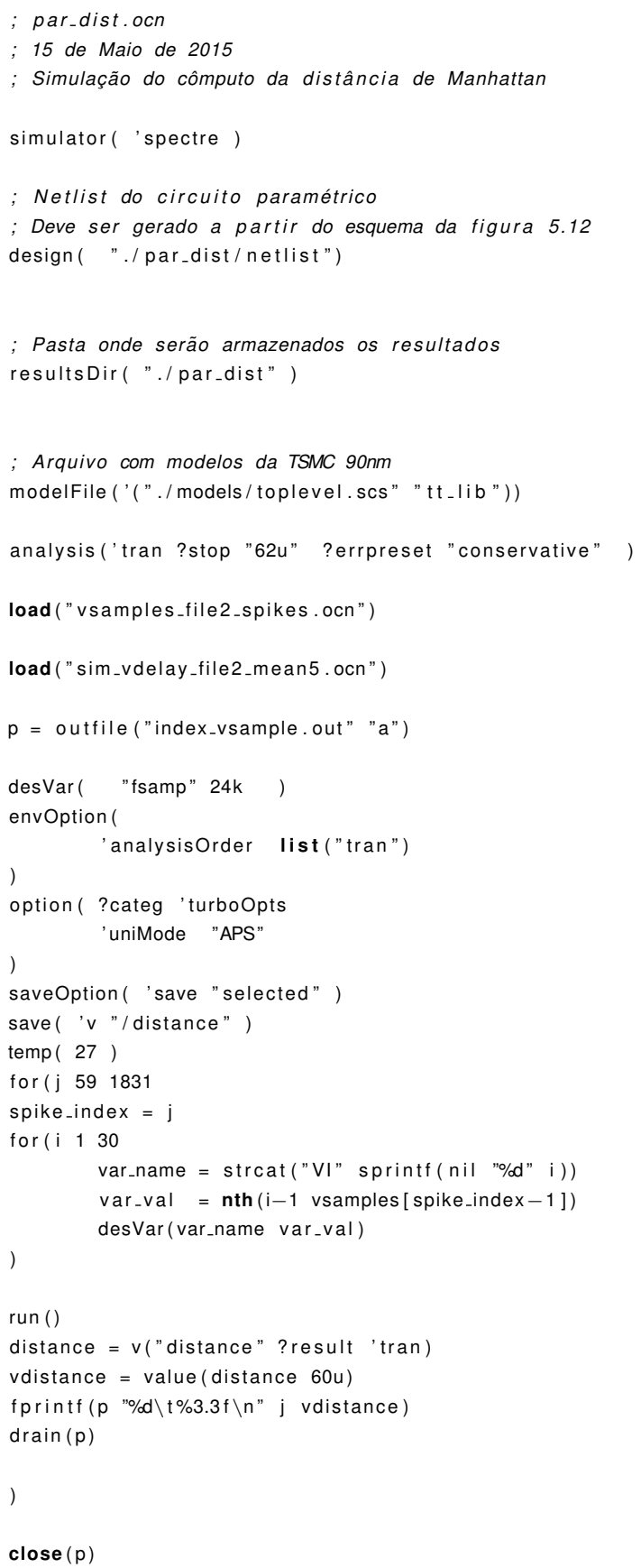




\section{Apêndice C - Esquemas elétricos em Cadence}

C.1 Esquema do circuito conversor de tensão a corrente

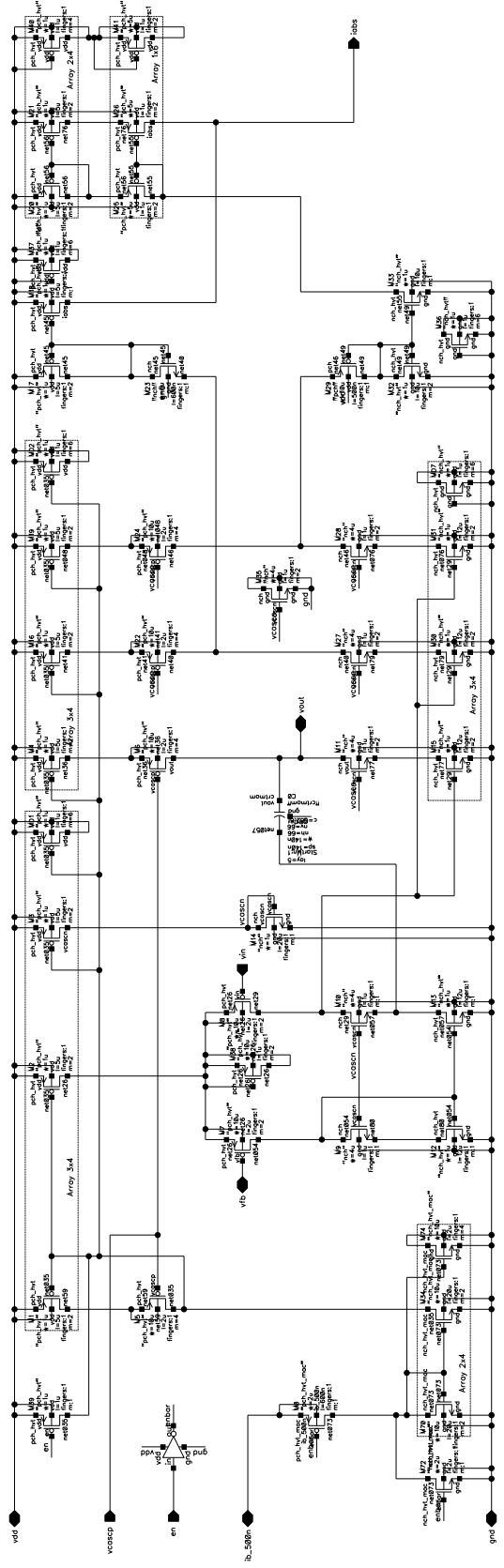

Figura C.1: Esquema do circuito conversor de tensão a corrente. 


\section{C.2 Esquema do circuito NEO}
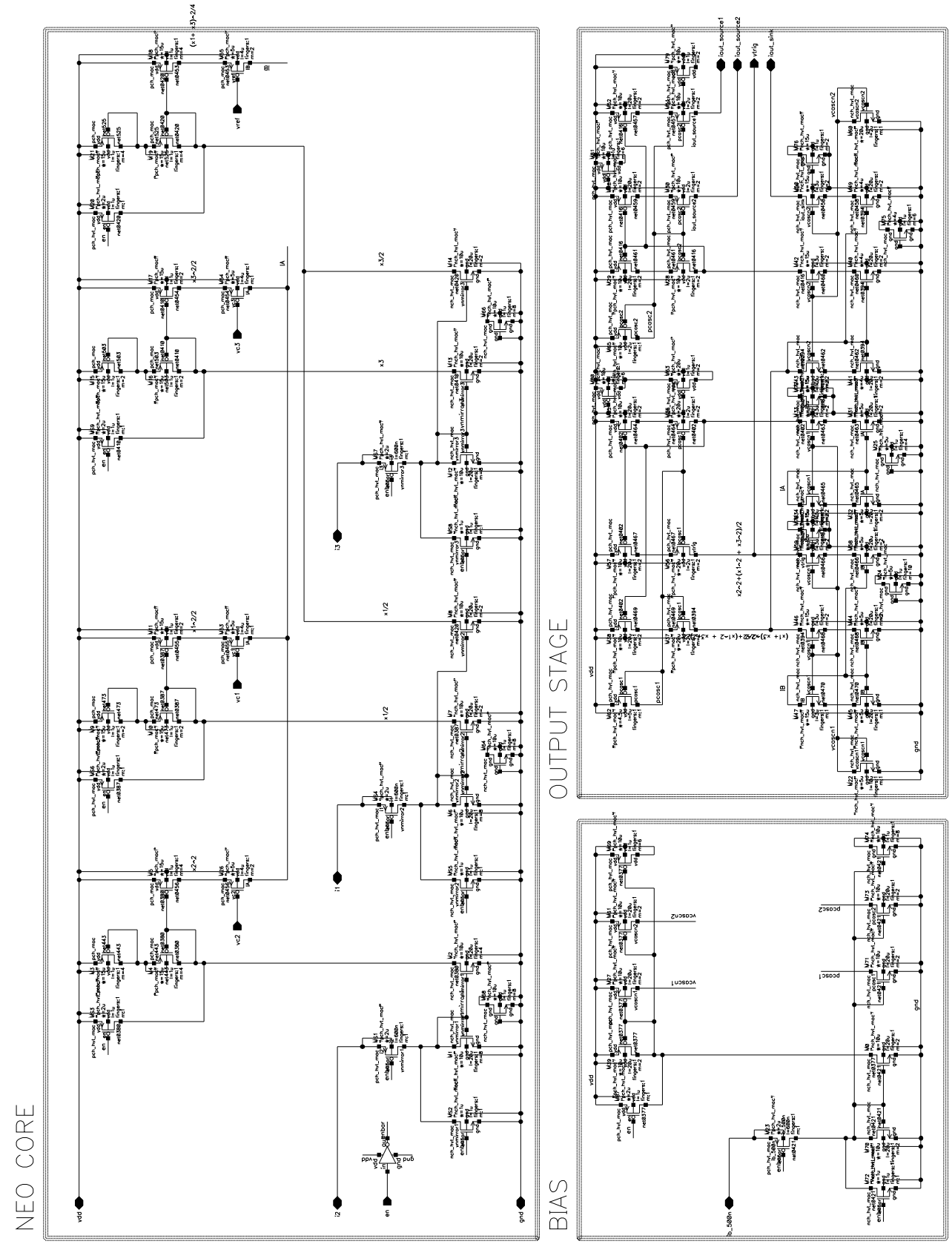

Figura C.2: Esquema do circuito NEO 


\section{Apêndice D - Layouts dos circuitos}

\section{D.1 Layout do circuito conversor de tensão a corrente}

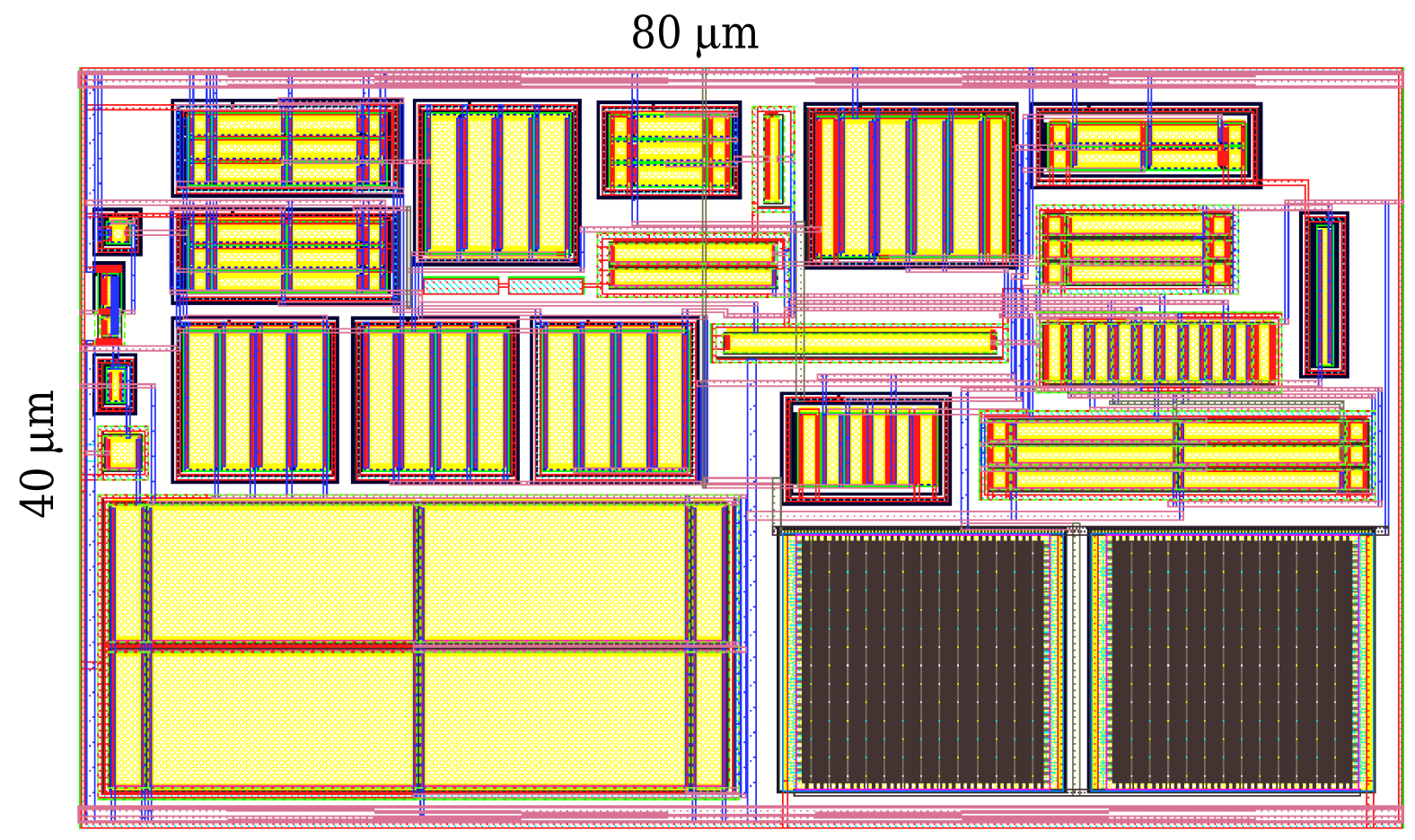

Figura D.1: Layout do circuito conversor de tensão a corrente. 


\section{D.2 Layout do circuito NEO}

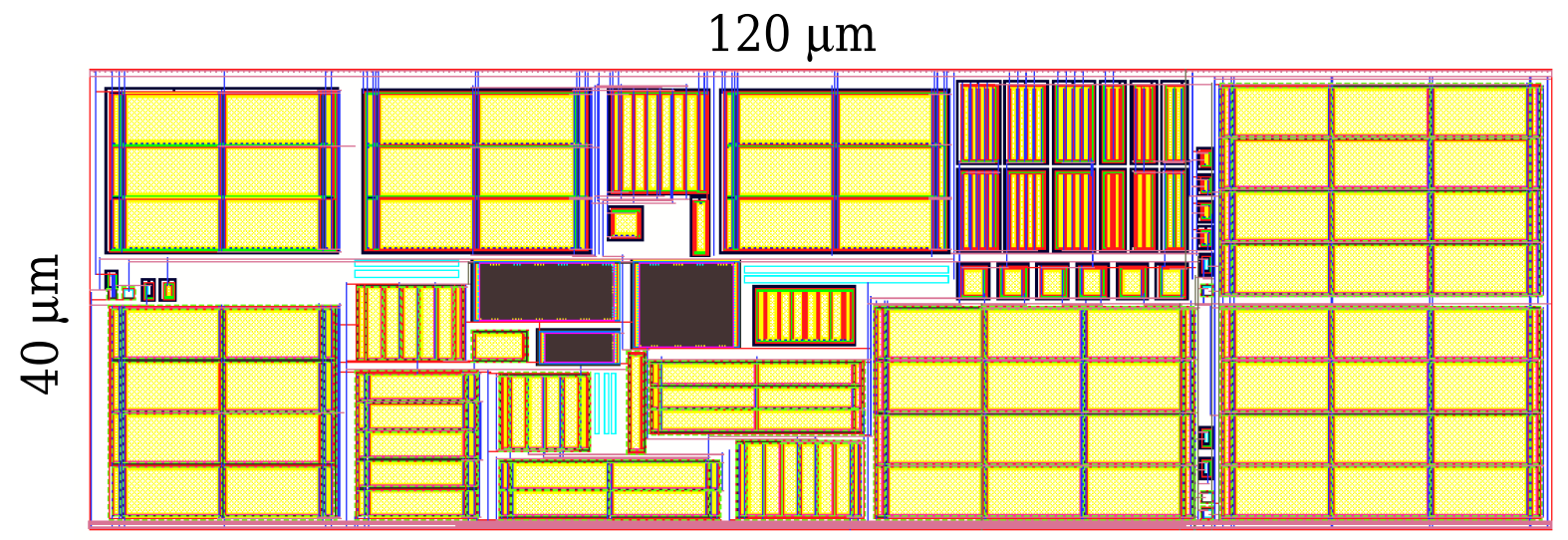

Figura D.2: Layout do circuito NEO 


\section{Apêndice E - Nestlists dos circuitos projetados}

A seguir apresenta-se os netlists dos circuitos projetados nesta tese, com o objetivo de viabilizar a reprodução dos resultados (através de uma operação copiar-colar seguida de simulação como o programa spectre da Cadence).

\section{E.1 Netlist do circuito NEO}

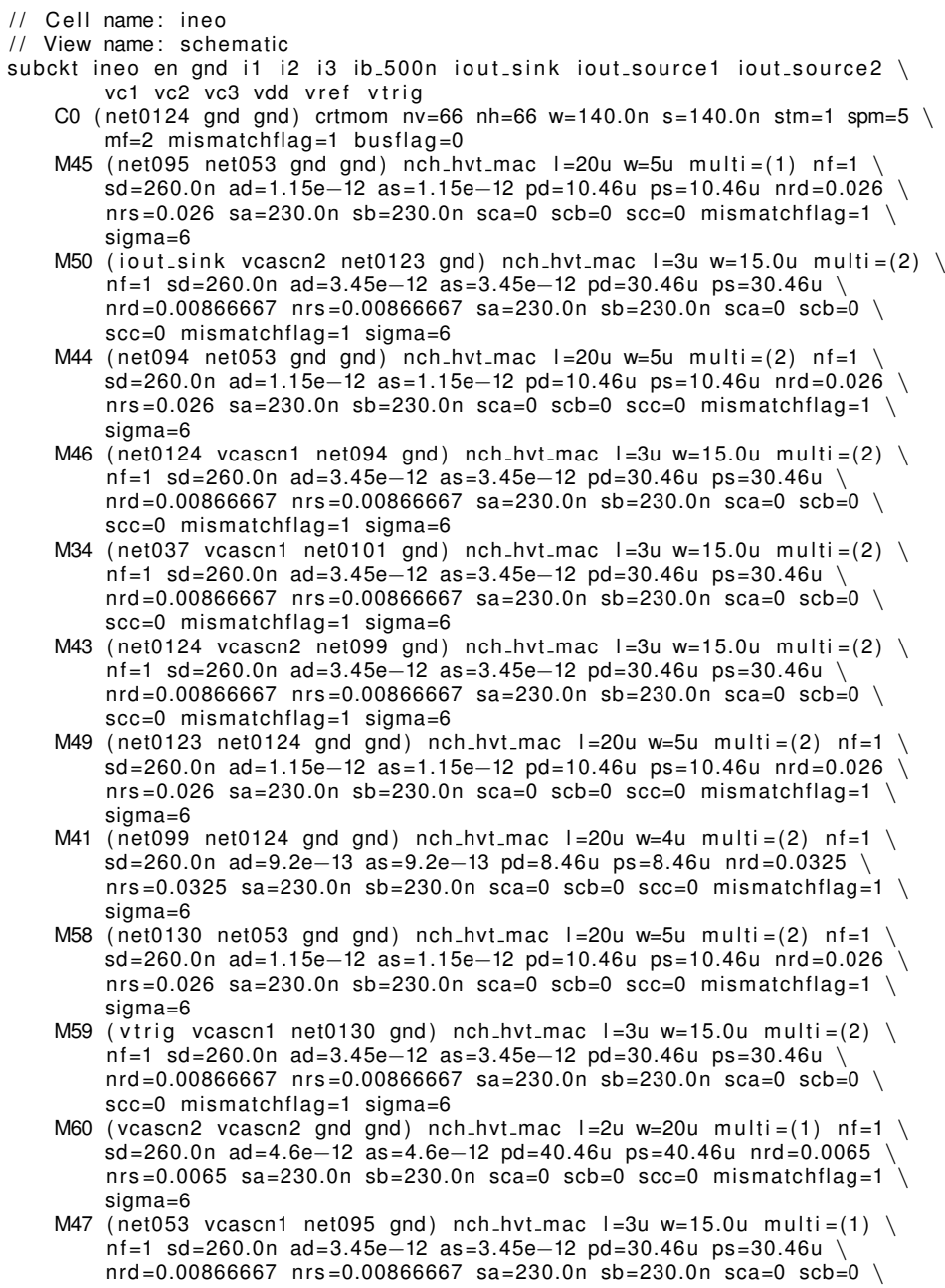

M44 (net094 net053 gnd gnd) nch_hvt_mac $I=20 u \quad w=5 u$ multi $=(2) \quad n f=1$ $\mathrm{sd}=260.0 \mathrm{n}$ ad $=1.15 \mathrm{e}-12$ as $=1.15 \mathrm{e}-12 \mathrm{pd}=10.46 \mathrm{u} \mathrm{ps}=10.46 \mathrm{u} \mathrm{nrd}=0.026$ $n r s=0.026 \mathrm{sa}=230.0 \mathrm{n} \quad \mathrm{sb}=230.0 \mathrm{n} \quad \mathrm{sca}=0 \mathrm{scb}=0 \mathrm{scc}=0$ mismatchflag=1 sigma $=6$

M46 (net0124 vcascn1 net094 gnd) nch_hvt_mac I=3u w=15.0u multi=(2) $\mathrm{nf}=1 \mathrm{sd}=260.0 \mathrm{n}$ ad $=3.45 \mathrm{e}-12$ as $=3.45 \mathrm{e}-12 \mathrm{pd}=30.46 \mathrm{u} \mathrm{ps}=30.46 \mathrm{u}$ $\mathrm{nrd}=0.00866667 \mathrm{nrs}=0.00866667 \mathrm{sa}=230.0 \mathrm{n} \mathrm{sb}=230.0 \mathrm{n} \quad \mathrm{sca}=0 \mathrm{scb}=0$ $\mathrm{scc}=0$ mismatchflag $=1$ sigma $=6$

M34 (net037 vcascn1 net0101 gnd) nch hvt mac $I=3 u w=15.0 u$ multi $=$ (2) $\mathrm{nf}=1 \mathrm{sd}=260.0 \mathrm{n}$ ad=3.45e-12 as $=3.45 \mathrm{e}-12 \mathrm{pd}=30.46 \mathrm{u}$ ps $=30.46 \mathrm{u}$ $\mathrm{nrd}=0.00866667 \mathrm{nrs}=0.00866667 \quad \mathrm{sa}=230.0 \mathrm{n} \quad \mathrm{sb}=230.0 \mathrm{n} \quad \mathrm{sca}=0 \quad \mathrm{scb}=0$ $\mathrm{scc}=0$ mismatchflag $=1$ sigma $=6$

M43 (net0124 vcascn2 net099 gnd) nch_hvt_mac $\mathrm{I}=3 \mathrm{u}$ w $=15.0 \mathrm{u}$ multi=(2) $\mathrm{nf}=1 \mathrm{sd}=260.0 \mathrm{n}$ ad $=3.45 \mathrm{e}-12$ as $=3.45 \mathrm{e}-12 \mathrm{pd}=30.46 \mathrm{u} \mathrm{ps}=30.46 \mathrm{u}$ $\mathrm{nrd}=0.00866667 \mathrm{nrs}=0.00866667 \quad \mathrm{sa}=230.0 \mathrm{n} \quad \mathrm{sb}=230.0 \mathrm{n}$ sca $=0 \quad \mathrm{scb}=0$ $\mathrm{scC}=0$ mismatchflag $=1$ sigma $=6$

M49 (net0123 net0124 gnd gnd) nch_hvt_mac $1=20 u \quad w=5 u$ multi=(2) nf=1 $\mathrm{sd}=260.0 \mathrm{n}$ ad $=1.15 \mathrm{e}-12$ as $=1.15 \mathrm{e}-12 \mathrm{pd}=10.46 \mathrm{u}$ ps $=10.46 \mathrm{u}$ nrd $=0.026$ $\mathrm{nrs}=0.026 \mathrm{sa}=230.0 \mathrm{n} \mathrm{sb}=230.0 \mathrm{n}$ sca $=0 \mathrm{scb}=0 \mathrm{scc}=0$ mismatchflag $=1$ sigma $=6$

M41 ( $\mathrm{sd}=260.0 \mathrm{n}$ ad $=9.2 \mathrm{e}-13$ as $=9.2 \mathrm{e}-13 \mathrm{pd}=8.46 \mathrm{u}$ ps $=8.46 \mathrm{u} \mathrm{nrd}=0.0325$ $\mathrm{nrs}=0.0325 \mathrm{sa}=230.0 \mathrm{n} \mathrm{sb}=230.0 \mathrm{n}$ sca $=0 \quad \mathrm{scb}=0 \quad \mathrm{scc}=0$ mismatchflag $=1$ sigma $=6$

M58 (net0130 net053 gnd gnd) nch hvt mac $I=20 u w=5 u$ multi $=(2) \quad n f=1$ $\mathrm{sd}=260.0 \mathrm{n}$ ad $=1.15 \mathrm{e}-12$ as $=1.15 \mathrm{e}-12 \mathrm{pd}=10.46 \mathrm{u} \mathrm{ps}=10.46 \mathrm{u} \mathrm{nrd}=0.026$ $\mathrm{nrs}=0.026 \mathrm{sa}=230.0 \mathrm{n} \quad \mathrm{sb}=230.0 \mathrm{n}$ sca $=0 \mathrm{scb}=0 \mathrm{scc}=0$ mismatchflag $=1$ sigma $=6$

M59 (vtrig vcascn1 net0130 gnd) nch_hvt_mac $\mathrm{I}=3 \mathrm{u} \mathrm{w}=15.0 \mathrm{u}$ multi=(2) $\mathrm{nf}=1 \mathrm{sd}=260.0 \mathrm{n}$ ad $=3.45 \mathrm{e}-12$ as $=3.45 \mathrm{e}-12 \mathrm{pd}=30.46 \mathrm{u}$ ps $=30.46 \mathrm{u}$ $\mathrm{nrd}=0.00866667 \mathrm{nrs}=0.00866667 \mathrm{sa}=230.0 \mathrm{n}$ sb=230.0n sca $=0 \quad \mathrm{scb}=0$ $\mathrm{scC}=0$ mismatchflag $=1$ sigma $=6$

M60 (vcascn2 vcascn2 gnd gnd) nch_hvt_mac $\mid=2 \mathrm{u} \quad \mathrm{W}=20 \mathrm{u}$ multi $=(1) \quad \mathrm{nt}=1$ $\mathrm{sd}=260.0 \mathrm{n}$ ad $=4.6 \mathrm{e}-12$ as $=4.6 \mathrm{e}-12 \mathrm{pd}=40.46 \mathrm{u} \mathrm{ps}=40.46 \mathrm{u} \mathrm{nrd}=0.0065$ $\mathrm{nrs}=0.0065 \mathrm{sa}=230.0 \mathrm{n} \quad \mathrm{sb}=230.0 \mathrm{n}$ sca $=0 \mathrm{scb}=0 \mathrm{scc}=0$ mismatchflag=1 sigma $=6$

M47 (net053 vcascn1 net095 gnd) nch_hvt_mac $I=3 \mathrm{u} w=15.0 \mathrm{u}$ multi $=(1)$ $\mathrm{nf}=1 \mathrm{sd}=260.0 \mathrm{n}$ ad $=3.45 \mathrm{e}-12$ as $=3.45 \mathrm{e}-12 \mathrm{pd}=30.46 \mathrm{u} \mathrm{ps}=30.46 \mathrm{u}$ $\mathrm{nrd}=0.00866667 \mathrm{nrs}=0.00866667 \mathrm{sa}=230.0 \mathrm{n} \quad \mathrm{sb}=230.0 \mathrm{n}$ sca $=0 \mathrm{scb}=0$ 
$\mathrm{scc}=0$ mismatchflag $=1$ sigma $=6$

M33 (net070 vcascn1 net0100 gnd) nch_hvt_mac I=3u $\mathrm{w}=15.0 \mathrm{u}$ multi=(2) $n f=1 \quad s d=260.0 n$ ad $=3.45 e-12$ as $=3.45 e-12$ pd=30.46u ps $=30.46 u$ $\mathrm{nrd}=0.00866667 \mathrm{nrs}=0.00866667 \mathrm{sa}=230.0 \mathrm{n} \quad \mathrm{sb}=230.0 \mathrm{n}$ sca $=0 \quad \mathrm{scb}=0$ $\mathrm{scC}=0$ mismatchflag $=1$ sigma $=6$

M42 (net085 vcascn2 net0104 gnd) nch hvt_mac $I=3 u$ w $=15.0 u$ multi $=(2)$ $\mathrm{nf}=1 \mathrm{sd}=260.0 \mathrm{n}$ ad $=3.45 \mathrm{e}-12$ as $=3.45 \mathrm{e}-12 \mathrm{pd}=30.46 \mathrm{u} \quad \mathrm{ps}=30.46 \mathrm{u}$

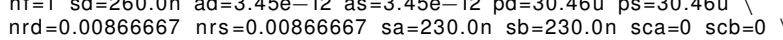
scc $=0$ mismatchflag $=1$ sigma $=6$

M40 (net0104 net0124 gnd gnd) nch_hvt_mac I=20u w=4u multi=(2) nf=1 \ $\mathrm{sd}=260.0 \mathrm{n}$ ad $=9.2 \mathrm{e}-13$ as $=9.2 \mathrm{e}-13 \mathrm{pd}=8.46 \mathrm{u} \mathrm{ps}=8.46 \mathrm{u} \mathrm{nrd}=0.0325$ $\mathrm{nrs}=0.0325 \mathrm{sa}=230.0 \mathrm{n} \mathrm{sb}=230.0 \mathrm{n}$ sca $=0 \mathrm{scb}=0 \mathrm{scc}=0$ mismatchflag=1 । sigma $=6$

M31 (net0100 net037 gnd gnd) nch_hvt_mac I=20u w=5u multi=(2) nf=1 $\mathrm{sd}=260.0 \mathrm{n}$ ad=1.15e -12 as $=1.15 \mathrm{e}-12 \mathrm{pd}=10.46 \mathrm{u}$ ps $=10.46 \mathrm{u}$ nrd $=0.026$ $\mathrm{nrs}=0.026 \mathrm{sa}=230.0 \mathrm{n} \quad \mathrm{sb}=230$. 0 n $\mathrm{sca}=0 \quad \mathrm{scb}=0 \quad \mathrm{scc}=0$ mismatchflag $=1$ । sigma $=6$

M14 (net043 vnmirror3 gnd gnd) nch_hvt_mac I=20u w=10u multi=(2) $n f=1$ । $\mathrm{sd}=260.0 \mathrm{n}$ ad $=2.3 \mathrm{e}-12$ as $=2.3 \mathrm{e}-12 \mathrm{pd}=20.46 \mathrm{u} \mathrm{ps}=20.46 \mathrm{u} \mathrm{nrd}=0.013$ $\mathrm{nrs}=0.013 \mathrm{sa}=230.0 \mathrm{n} \mathrm{sb}=230.0 \mathrm{n} \quad \mathrm{sca}=0 \mathrm{scb}=0 \mathrm{scc}=0$ mismatchflag $=1$ sigma $=6$

M12 (vnmirror3 vnmirror3 gnd gnd) nch_hvt_mac $\mathrm{I}=20 \mathrm{u}$ w $=10 \mathrm{u}$ multi $=(8)$ ) $n f=1 \quad s d=260.0 n$ ad $=2.3 e-12$ as $=2.3 e-12$ pd $=20.46 u$ ps $=20.46 u$ nrd $=0.013$ $\mathrm{nrs}=0.013$ sa $=230.0 n$ sb $=230.0$ n sca $=0 \quad$ scb $=0 \quad$ scc $=0$ mismatchflag $=1$ । $\mathrm{nrs}=0.013$
sigma $=6$

M13 (net0105 vnmirror3 gnd gnd) nch_hvt_mac $\mathrm{I}=20 \mathrm{u} \mathrm{w}=10 \mathrm{u}$ multi=(4) $\mathrm{nf}=1$ $\mathrm{sd}=260.0 \mathrm{n}$ ad $=2.3 \mathrm{e}-12$ as $=2.3 \mathrm{e}-12 \mathrm{pd}=20.46 \mathrm{u} \mathrm{ps}=20.46 \mathrm{u} \mathrm{nrd}=0.013$ $\mathrm{nrs}=0.013 \mathrm{sa}=230.0 \mathrm{n} \mathrm{sb}=230.0 \mathrm{n} \quad \mathrm{sca}=0 \mathrm{scb}=0 \mathrm{scc}=0$ mismatchflag $=1$ sigma $=6$

MS8 (vnmirror3 enbar gnd gnd) nch_hvt_mac $\mathrm{I}=1 \mathrm{u} \quad \mathrm{w}=1 \mathrm{u}$ multi $=(4) \mathrm{nf}=1$ ) $\mathrm{sd}=260.0 \mathrm{n} \mathrm{ad}=2.3 \mathrm{e}-13$ as $=2.3 \mathrm{e}-13 \mathrm{pd}=2.46 \mathrm{u} \mathrm{ps}=2.46 \mathrm{u} \mathrm{nrd}=0.13$ $\mathrm{nrs}=0.13 \mathrm{sa}=230.0 \mathrm{n} \mathrm{sb}=230.0 \mathrm{n}$ sca $=0 \mathrm{scb}=0 \mathrm{scc}=0$ mismatchflag $=1$ । sigma $=6$

M6 (vnmirror2 vnmirror2 gnd gnd) nch_hvt_mac $\mathrm{I}=20 \mathrm{u} \mathrm{w}=10 \mathrm{u}$ multi $=(8)$ $n f=1 \quad s d=260.0 n$ ad $=2.3 e-12$ as $=2.3 e-12$ pd $=20.46 u$ ps $=20.46 u$ nrd $=0.013$ $n r s=0.013 \mathrm{sa}=230.0 \mathrm{n} \mathrm{sb}=230.0 \mathrm{n} \mathrm{sca}=0 \mathrm{scb}=0 \mathrm{scc}=0$ mismatchflag $=1$ । sigma $=6$

M8 (net043 vnmirror2 gnd gnd) nch_hvt_mac $\mathrm{I}=20 \mathrm{u} \mathrm{w}=10 \mathrm{u}$ multi $=(2) \mathrm{nf}=1$ $\mathrm{sd}=260.0 \mathrm{n}$ ad $=2.3 \mathrm{e}-12$ as $=2.3 \mathrm{e}-12 \mathrm{pd}=20.46 \mathrm{u} \mathrm{ps}=20.46 \mathrm{u} \mathrm{nrd}=0.013$ $\mathrm{sd}=260.0 \mathrm{n}$ ad $=2.3 \mathrm{e}-12 \mathrm{as}=2.3 \mathrm{e}-12 \mathrm{pd}=20.46 \mathrm{u} \mathrm{ps}=20.46 \mathrm{u}$ nrd $=0.013$ $\mathrm{nrs}=0.013$
sigma $=6$

M7 (net098 vnmirror2 gnd gnd) nch_hvt_mac $I=20 \mathrm{u} w=10 \mathrm{u}$ multi $=(4) \quad \mathrm{nf}=1$ $\mathrm{sd}=260.0 \mathrm{n} \mathrm{ad}=2.3 \mathrm{e}-12$ as $=2.3 \mathrm{e}-12 \mathrm{pd}=20.46 \mathrm{u}$ ps $=20.46 \mathrm{u} \mathrm{nrd}=0.013$ $\mathrm{nrs}=0.013 \mathrm{sa}=230.0 \mathrm{n} \mathrm{sb}=230.0 \mathrm{n}$ sca $=0 \mathrm{scb}=0 \mathrm{scc}=0$ mismatchflag=1 sigma $=6$

M1 (vnmirror1 vnmirror1 gnd gnd) nch_hvt_mac $\mathrm{I}=20 \mathrm{u} \mathrm{w}=10 \mathrm{u}$ multi $=(8)$ $\mathrm{nf}=1 \quad \mathrm{sd}=260.0 \mathrm{n}$ ad $=2.3 \mathrm{e}-12$ as $=2.3 \mathrm{e}-12 \mathrm{pd}=20.46 \mathrm{u} \quad \mathrm{ps}=20.46 \mathrm{u}$ nrd $=0.013$ $\mathrm{nrs}=0.013 \mathrm{sa}=230.0 \mathrm{n}$ sb $=230$.0n $\mathrm{sca}=0 \quad \mathrm{scb}=0 \quad \mathrm{scc}=0$ mismatchflag $=1$ । sigma $=6$

M2 (net092 vnmirror1 gnd gnd) nch_hvt_mac $\mathrm{I}=20 \mathrm{u} \mathrm{w=10u}$ multi $=(4) \quad \mathrm{nf}=1$ $\mathrm{sd}=260.0 \mathrm{n}$ ad $=2.3 \mathrm{e}-12$ as $=2.3 \mathrm{e}-12 \mathrm{pd}=20.46 \mathrm{u} \mathrm{ps}=20.46 \mathrm{u} \mathrm{nrd}=0.013$ । $\mathrm{nrs}=0.013 \mathrm{sa}=230.0 \mathrm{n} \mathrm{sb}=230.0 \mathrm{n} \mathrm{sca}=0 \mathrm{scb}=0 \mathrm{scc}=0$ mismatchflag $=1$ sigma $=6$

MS5 (vnmirror2 enbar gnd gnd) nch_hvt_mac $I=1 \mathrm{u} w=1 \mathrm{u}$ multi=(4) $\mathrm{nf}=1$ $\mathrm{sd}=260.0 \mathrm{n}$ ad $=2.3 \mathrm{e}-13$ as $=2.3 \mathrm{e}-13 \mathrm{pd}=2.46 \mathrm{u}$ ps $=2.46 \mathrm{u} \mathrm{nrd}=0.13$ $\mathrm{nrs}=0.13 \mathrm{sa}=230.0 \mathrm{n} \quad \mathrm{sb}=230.0 \mathrm{n} \quad \mathrm{sca}=0 \quad \mathrm{scb}=0 \quad \mathrm{scc}=0$ mismatchflag $=1$ $\mathrm{nrs}=0.13$
sigma $=6$

M22 (vascn1 vcascn1 and gnd) nch hvt mac $l=10 u w=5 u$ multi $=(1) \quad n f=1$ Y $\mathrm{sd}=260.0 \mathrm{n}$ ad $=1.15 \mathrm{e}-12$ as $=1.15 \mathrm{e}-12 \mathrm{pd}=10.46 \mathrm{u}$ ps $=10.46 \mathrm{u}$ nrd $=0.026$ $\mathrm{nrs}=0.026 \mathrm{sa}=230.0 \mathrm{n}$ sb=230.0n sca $=0$ scb $=0$ scc $=0$ mismatchflag $=1$ । sigma $=6$

M32 (net0101 net037 gnd gnd) nch_hvt_mac $\mathrm{I}=20 \mathrm{u} w=5 \mathrm{u}$ multi $=(2) \mathrm{nf}=1$ > $\mathrm{sd}=260.0 \mathrm{n}$ ad $=1.15 \mathrm{e}-12$ as $=1.15 \mathrm{e}-12 \mathrm{pd}=10.46 \mathrm{u}$ ps $=10.46 \mathrm{u}$ nrd $=0.026$ $\mathrm{nrs}=0.026 \mathrm{sa}=230.0 \mathrm{n} \quad \mathrm{sb}=230.0 \mathrm{n} \quad \mathrm{sca}=0 \quad \mathrm{scb}=0 \quad \mathrm{scc}=0$ mismatchflag $=1 \backslash$ sigma $=6$

MS2 (vnmirror1 enbar gnd gnd) nch_hvt_mac $I=1 \mathrm{u} \quad \mathrm{w}=1 \mathrm{u}$ multi $=(4) \mathrm{nf}=1$ $\mathrm{sd}=260.0 \mathrm{n}$ ad $=2.3 \mathrm{e}-13 \mathrm{as}=2.3 \mathrm{e}-13 \mathrm{pd}=2.46 \mathrm{u} \mathrm{ps}=2.46 \mathrm{u} \mathrm{nrd}=0.13$ $\mathrm{nrs}=0.13 \mathrm{sa}=230.0 \mathrm{n} \mathrm{sb}=230.0 \mathrm{n}$ sca $=0 \mathrm{scb}=0 \mathrm{scc}=0$ mismatchflag=1 sigma $=6$

M27 (vcascn1 ib_500n vdd vdd) pch_hvt_mac $\mathrm{I}=5 \mathrm{u} \quad \mathrm{w}=2.5 \mathrm{u}$ multi $=(2) \mathrm{nf}=1$ $\mathrm{sd}=260.0 \mathrm{n} \mathrm{ad}=5.75 \mathrm{e}-13$ as $=5.75 \mathrm{e}-13 \mathrm{pd}=5.46 \mathrm{u}$ ps $=5.46 \mathrm{u} \mathrm{nrd}=0.052$ $\mathrm{nrs}=0.052 \mathrm{sa}=230.0 \mathrm{n} \mathrm{sb}=230.0 \mathrm{n} \mathrm{sca}=0 \mathrm{scb}=0 \mathrm{scc}=0$ mismatchflag=1 $\mathrm{nrs}=0.052$
sigma $=6$

M29 (net0122 net085 vdd vdd) pch_hvt_mac $\mathrm{I}=20 \mathrm{u}$ w=1u multi $=(2) \mathrm{nf}=1$ $\mathrm{sd}=260.0 \mathrm{n}$ ad=2.3e-13 as $=2.3 \mathrm{e}-13 \mathrm{pd}=2.46 \mathrm{u} \mathrm{ps}=2.46 \mathrm{u} \mathrm{nrd}=0.13$ । $\mathrm{sd}=260.0 \mathrm{n} \quad \mathrm{ad}=2.3 \mathrm{e}-13$ as $=2.3 \mathrm{e}-13 \mathrm{pd}=2.46 \mathrm{u} \mathrm{ps}=2.46 \mathrm{u} \mathrm{nrd}=0.13$
$\mathrm{nrs}=0.13$ sa $=230.0 \mathrm{n}$ sb $=230.0 \mathrm{n}$ sca $=0 \quad \mathrm{scb}=0 \quad \mathrm{scc}=0$ mismatchflag $=1$ $\mathrm{nrs}=0.13$
sigma $=6$

M30 (iout_source2 ib_500n net0121 vdd) pch_hvt_mac $I=5 \mathrm{u} \quad \mathrm{w}=20 \mathrm{u}$ multi $=$ (2) $n f=1 \quad s d=260.0 n$ ad $=4.6 e-12$ as $=4.6 e-12$ pd=40.46u ps $=40.46 u$ $\mathrm{nrd}=0.0065 \mathrm{nrs}=0.0065$ sa $=230.0 \mathrm{n} \quad \mathrm{sb}=230.0 \mathrm{n} \quad \mathrm{sca}=0 \quad \mathrm{scb}=0 \quad \mathrm{scc}=0$ । mismatchflag $=1$ sigma $=6$

M48 (net0121 net085 vdd vdd) pch_hvt_mac $\mathrm{I}=20 \mathrm{u} w=1 \mathrm{u}$ multi=(2) $\mathrm{nf}=1$ > $\mathrm{sd}=260.0 \mathrm{n} \mathrm{ad}=2.3 \mathrm{e}-13$ as $=2.3 \mathrm{e}-13 \mathrm{pd}=2.46 \mathrm{u}$ ps $=2.46 \mathrm{u} \mathrm{nrd}=0.13$ $\mathrm{nrs}=0.13 \mathrm{sa}=230.0 \mathrm{n} \mathrm{sb}=230.0 \mathrm{n} \quad \mathrm{sca}=0 \mathrm{scb}=0 \mathrm{scc}=0$ mismatchflag=1 । sigma $=6$

M51 (iout_source1 ib_500n net0116 vdd) pch_hvt_mac I=5u w=20u multi=(2) $n f=1 \quad s d=260.0 n$ ad $=4.6 e-12$ as $=4.6 e-12$ pd=40.46u ps $=40.46 u$ $\mathrm{nrd}=0.0065 \mathrm{nrs}=0.0065 \mathrm{sa}=230.0 \mathrm{n} \mathrm{sb}=230.0 \mathrm{n} \mathrm{sca}=0 \mathrm{scb}=0 \mathrm{scc}=0$ mismatchflag $=1$ sigma $=6$

M39 (ib_500n ib_500n vdd vdd) pch_hvt_mac $I=5 \mathrm{u} w=2.5 \mathrm{u}$ multi=(2) $n f=1$ $\mathrm{sd}=260.0 \mathrm{n}$ ad $=5.75 \mathrm{e}-13$ as $=5.75 \mathrm{e}-13 \mathrm{pd}=5.46 \mathrm{u}$ ps $=5.46 \mathrm{u} \mathrm{nrd}=0.052$ $\mathrm{nrs}=0.052 \mathrm{sa}=230.0 \mathrm{n} \mathrm{sb}=230.0 \mathrm{n} \quad \mathrm{sca}=0 \mathrm{scb}=0 \quad \mathrm{scc}=0$ mismatchflag $=1$ sigma $=6$

M52 (net0116 net085 vdd vdd) pch_hvt_mac $\mathrm{I}=20 \mathrm{u} w=1 \mathrm{u}$ multi $=(2) \mathrm{nf}=1$ > $\mathrm{sd}=260.0 \mathrm{n}$ ad $=2.3 \mathrm{e}-13 \mathrm{as}=2.3 \mathrm{e}-13 \mathrm{pd}=2.46 \mathrm{u} \mathrm{ps}=2.46 \mathrm{u} \mathrm{nrd}=0.13$ । $\mathrm{nrs}=0.13 \mathrm{sa}=230.0 \mathrm{n} \mathrm{sb}=230.0 \mathrm{n}$ sca $=0 \mathrm{scb}=0 \mathrm{scc}=0$ mismatchflag=1 । sigma $=6$

M35 (net097 net070 vdd vdd) pch_hvt_mac $\quad \mathrm{I}=20 \mathrm{u}$ w=8u multi=(2) nf=1 । 
$\mathrm{sd}=260.0 \mathrm{n}$ ad $=1.84 \mathrm{e}-12$ as $=1.84 \mathrm{e}-12 \mathrm{pd}=16.46 \mathrm{u} \mathrm{ps}=16.46 \mathrm{u} \mathrm{nrd}=0.01625$ । $\mathrm{nrs}=0.01625 \mathrm{sa}=230.0 \mathrm{n} \mathrm{sb}=230$. 0 n sca $=0 \mathrm{scb}=0$ scc=0 mismatchflag=1 \} sigma $=6$

M38 (net096 net070 vdd vdd) pch_hvt_mac $\mathrm{I}=20 \mathrm{u}$ w=8u multi=(2) $\mathrm{nf}=1$ $\mathrm{sd}=260.0 \mathrm{n}$ ad $=1.84 \mathrm{e}-12$ as $=1.84 \mathrm{e}-12 \mathrm{pd}=16.46 \mathrm{u} \mathrm{ps}=16.46 \mathrm{u} \mathrm{nrd}=0.01625$ $\mathrm{nrs}=0.01625$ sa=230.0n $\mathrm{sb}=230.0 \mathrm{n} \quad \mathrm{sca}=0 \quad \mathrm{scb}=0$ scc=0 mismatchflag=1 sigma $=6$

M37 (net0124 ib 500n net096 vdd) pch hvt mac $\mathrm{I}=3 \mathrm{u}$ w=15.0u multi $=(2)$ $\mathrm{nf}=1 \quad \mathrm{sd}=260.0 \mathrm{n}$ ad $=3.45 \mathrm{e}-12$ as $=3.45 \mathrm{e}-12 \mathrm{pd}=30.46 \mathrm{u}$ ps $=30.46 \mathrm{u}$ $\mathrm{nrd}=0.00866667 \mathrm{nrs}=0.00866667 \quad \mathrm{sa}=230.0 \mathrm{n} \quad \mathrm{sb}=230.0 \mathrm{n} \quad \mathrm{sca}=0 \quad \mathrm{scb}=0$ $\mathrm{scC}=0$ mismatchflag $=1$ sigma $=6$

M56 (vtrig ib_500n net0131 vdd) pch_hvt_mac $I=3 \mathrm{u} w=15.0 \mathrm{u}$ multi=(2) $\mathrm{nf}=1 \quad \mathrm{sd}=260.0 \mathrm{n}$ ad=3.45e -12 as $=3.45 \mathrm{e}-12 \mathrm{pd}=30.46 \mathrm{u}$ ps $=30.46 \mathrm{u}$ $\mathrm{nrd}=0.00866667 \mathrm{nrs}=0.00866667 \mathrm{sa}=230.0 \mathrm{n} \mathrm{sb}=230.0 \mathrm{n} \mathrm{sca}=0 \quad \mathrm{scb}=0$ $\mathrm{scc}=0$ mis matchflag $=1$ sigma $=6$

M57 (net0131 net070 vdd vdd) pch_hvt_mac $I=20 u \quad w=8 u$ multi $=(2) \quad n f=1$ $\mathrm{sd}=260.0 \mathrm{n}$ ad $=1.84 \mathrm{e}-12$ as $=1.84 \mathrm{e}-12 \mathrm{pd}=16.46 \mathrm{u}$ ps $=16.46 \mathrm{u}$ nrd $=0.01625$ $\mathrm{nrs}=0.01625 \mathrm{sa}=230.0 \mathrm{n} \mathrm{sb}=230.0 \mathrm{n} \mathrm{sca}=0 \mathrm{scb}=0 \mathrm{scc}=0$ mismatchflag=1 sigma $=6$

M61 (vcascn2 ib_500n vdd vdd) pch_hvt_mac $I=5 u \quad w=2.5 u$ multi $=(2) \quad n f=1$ $\mathrm{sd}=260.0 \mathrm{n}$ ad $=5.75 \mathrm{e}-13$ as $=5.75 \mathrm{e}-13 \mathrm{pd}=5.46 \mathrm{u} \mathrm{ps}=5.46 \mathrm{u} \mathrm{nrd}=0.052$ $\mathrm{nrs}=0.052 \mathrm{sa}=230.0 \mathrm{n} \mathrm{sb}=230.0 \mathrm{n}$ sca $=0 \mathrm{scb}=0 \mathrm{scc}=0$ mismatchflag $=1$ sigma $=6$

M36 (net070 ib_500n net097 vdd) pch_hvt_mac $\mathrm{I}=3 \mathrm{u}$ w=15.0u multi $=(2)$ $\mathrm{nf}=1 \mathrm{sd}=260.0 \mathrm{n}$ ad $=3.45 \mathrm{e}-12$ as $=3.45 \mathrm{e}-12 \mathrm{pd}=30.46 \mathrm{u}$ ps $=30.46 \mathrm{u}$

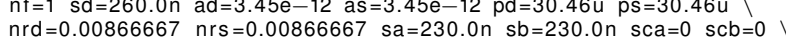
$\mathrm{scc}=0$ mismatchflag $=1$ sigma $=6$

M20 (net043 en vdd vdd) pch_hvt_mac $I=600 \mathrm{n} w=2 u$ multi $=(1) \quad n f=1$ ) $\mathrm{sd}=260.0 \mathrm{n}$ ad $=4.6 \mathrm{e}-13$ as $=4.6 \mathrm{e}-13 \mathrm{pd}=4.46 \mathrm{u}$ ps $=4.46 \mathrm{u}$ nrd $=0.065$ $\mathrm{nrs}=0.065 \mathrm{sa}=230.0 \mathrm{n} \mathrm{sb}=230.0 \mathrm{n}$ sca $=0 \quad \mathrm{scb}=0$ scc $=0$ mismatchflag $=1$ sigma $=6$

MS7 (vnmirror3 enbar i3 i3) pch_hvt_mac $\mathrm{I}=600 \mathrm{n} \mathrm{w}=2 \mathrm{u}$ multi $=(1) \mathrm{nf}=1$ $\mathrm{sd}=260.0 \mathrm{n}$ ad $=4.6 \mathrm{e}-13$ as $=4.6 \mathrm{e}-13 \mathrm{pd}=4.46 \mathrm{u} \mathrm{ps}=4.46 \mathrm{u} \mathrm{nrd}=0.065$ $n r s=0.065 \mathrm{sa}=230.0 \mathrm{n} \quad \mathrm{sb}=230.0 \mathrm{n}$ sca $=0 \mathrm{scb}=0 \mathrm{scc}=0$ mismatchflag $=1$ sigma $=6$

MS4 (vnmirror2 enbar i1 i1) pch_hvt_mac $I=600 \mathrm{n} w=2 u$ multi $=(1) \mathrm{nf}=1$ । $\mathrm{sd}=260.0 \mathrm{n}$ ad $=4.6 \mathrm{e}-13$ as $=4.6 \mathrm{e}-13 \mathrm{pd}=4.46 \mathrm{u} \mathrm{ps}=4.46 \mathrm{u} \mathrm{nrd}=0.065$ $\mathrm{nrs}=0.065 \mathrm{sa}=230.0 \mathrm{n} \mathrm{sb}=230.0 \mathrm{n} \quad \mathrm{sca}=0 \mathrm{scb}=0 \mathrm{scc}=0$ mismatchflag $=1$ sigma $=6$

Msg (net0105

en vdd vdd) pch_hvt_mac $I=600 \mathrm{n} \quad \mathrm{w}=2 \mathrm{u}$ multi $=(1) \quad \mathrm{nf}=1$ $\mathrm{sd}=260.0 \mathrm{n}$ ad $=4.6 \mathrm{e}-13$ as $=4.6 \mathrm{e}-13 \mathrm{pd}=4.46 \mathrm{u}$ ps $=4.46 \mathrm{u} \mathrm{nrd}=0.065$ $\mathrm{nrs}=0.065 \mathrm{sa}=230.0 \mathrm{n} \quad \mathrm{sb}=230.0 \mathrm{n} \quad \mathrm{sca}=0 \quad \mathrm{scb}=0 \quad \mathrm{scc}=0$ mismatchflag $=1$ sigma $=6$

MS1 (vnmirror1 enbar i2 i2) pch_hvt_mac $\quad l=600 n \quad w=2 u$ multi $=(1) \quad n f=1$ $\mathrm{sd}=260.0 \mathrm{n}$ ad $=4.6 \mathrm{e}-13$ as $=4.6 \mathrm{e}-13 \mathrm{pd}=4.46 \mathrm{u}$ ps $=4.46 \mathrm{u}$ nrd $=0.065$ $\mathrm{nrs}=0.065 \mathrm{sa}=230.0 \mathrm{n} \mathrm{sb}=230.0 \mathrm{n}$ sca $=0 \quad \mathrm{scb}=0$ scc $=0$ mismatchflag $=1$ sigma $=6$

MS6 ( $\mathrm{sd}=260.0 \mathrm{n}$ ad $=4.6 \mathrm{e}-13$ as $=4.6 \mathrm{e}-13 \mathrm{pd}=4.46 \mathrm{u}$ ps $=4.46 \mathrm{u}$ nrd $=0.065$ $\mathrm{nrs}=0.065 \mathrm{sa}=230.0 \mathrm{n} \mathrm{sb}=230.0 \mathrm{n} \mathrm{sca}=0 \mathrm{scb}=0 \mathrm{scc}=0$ mismatchflag $=1$ । sigma $=6$

MS3 ( net092 en vdd vdd) pch_hvt_mac $I=600 \mathrm{n} w=2 u$ multi $=(1) \mathrm{nf}=1$ $\mathrm{sd}=260.0 \mathrm{n}$ ad $=4.6 \mathrm{e}-13$ as $=4.6 \mathrm{e}-13 \mathrm{pd}=4.46 \mathrm{u} \mathrm{ps}=4.46 \mathrm{u} \mathrm{nrd}=0.065$ $\mathrm{nrs}=0.065 \mathrm{sa}=230.0 \mathrm{n} \mathrm{sb}=230.0 \mathrm{n} \quad \mathrm{sca}=0 \mathrm{scb}=0 \mathrm{scc}=0$ mismatchflag $=1$ sigma $=6$

M28 (net085 ib_500n net0122 vdd) pch_hvt_mac I $=5 \mathrm{u} w=20 \mathrm{u}$ multi=(2) $n f=1$ V $\mathrm{sd}=260.0 \mathrm{n}$ ad $=4.6 \mathrm{e}-12$ as $=4.6 \mathrm{e}-12 \mathrm{pd}=40.46 \mathrm{u}$ ps $=40.46 \mathrm{u} \mathrm{nrd}=0.0065$ $\mathrm{sd}=260.0 \mathrm{n}$ ad $=4.6 \mathrm{e}-12$ as $=4.6 \mathrm{e}-12 \mathrm{pd}=40.46 \mathrm{u} \mathrm{ps}=40.46 \mathrm{u} \mathrm{nrd}=0.0065$
$\mathrm{nrs}=0.0065$ sa $=230.0 \mathrm{n} \quad \mathrm{sb}=230.0 \mathrm{n}$ sca $=0 \quad \mathrm{scb}=0 \quad \mathrm{scc}=0$ mismatchflag $=1$ $\mathrm{nrs}=0.006$

110 (gnd en enbar vdd) inv hvt

M26 (net037 vc2 net027 vdd) pch_mac $I=4 \mathrm{u} \quad \mathrm{w}=5 \mathrm{u}$ multi=(2) $\mathrm{nf}=1 \mathrm{sd}=260.0 \mathrm{n}$ $\mathrm{ad}=1.15 \mathrm{e}-12$ as $=1.15 \mathrm{e}-12 \mathrm{pd}=10.46 \mathrm{u} \mathrm{ps}=10.46 \mathrm{u} \mathrm{nrd}=0.026 \mathrm{nrs}=0.026$ $\mathrm{sa}=230.0 \mathrm{n} \quad \mathrm{sb}=230$. $0 \mathrm{n} \quad \mathrm{sca}=0 \quad \mathrm{scb}=0 \quad \mathrm{scc}=0$ mismatchflag $=1$ sigma $=6$

M53 (net037 vc1 net0140 vdd) pch_mac $I=4 \mathrm{u} \quad w=5 \mathrm{u}$ multi=(1) $\mathrm{nf}=1$ $\mathrm{sd}=260.0 \mathrm{n}$ ad $=1.15 \mathrm{e}-12$ as $=1.15 \mathrm{e}-12 \mathrm{pd}=10.46 \mathrm{u} \mathrm{ps}=10.46 \mathrm{u}$ nrd $=0.026$ $\mathrm{nrs}=0.026 \mathrm{sa}=230.0 \mathrm{n} \quad \mathrm{sb}=230$. 0 n sca $=0 \quad \mathrm{scb}=0$ scc $=0$ mismatchflag $=1$ । sigma $=6$

M54 (net037 vc3 net0120 vdd) pch_mac I=4u $w=5 u$ multi $=(1) \quad n f=1$ ) $\mathrm{sd}=260.0 \mathrm{n}$ ad=1.15e -12 as $=1.15 \mathrm{e}-12 \mathrm{pd}=10.46 \mathrm{u} \mathrm{ps}=10.46 \mathrm{u} \mathrm{nrd}=0.026 \backslash$ $\mathrm{nrs}=0.026 \mathrm{sa}=230.0 \mathrm{n} \mathrm{sb}=230.0 \mathrm{n}$ sca $=0 \mathrm{scb}=0 \mathrm{scc}=0$ mismatchflag=1 sigma $=6$

M55 (net053 vref net0119 vdd) pch_mac $I=4 u \quad w=5 u$ multi $=$ (2) $n f=1$ $\mathrm{sd}=260.0 \mathrm{n}$ ad $=1.15 \mathrm{e}-12$ as $=1.15 \mathrm{e}-12 \mathrm{pd}=10.46 \mathrm{u} \mathrm{ps}=10.46 \mathrm{u} \mathrm{nrd}=0.026$ $\mathrm{sd}=260.0 \mathrm{n}$ ad $=1.15 \mathrm{e}-12$ as $=1.15 \mathrm{e}-12 \mathrm{pd}=10.46 \mathrm{u} \mathrm{ps}=10.46 \mathrm{u}$ nrd $=0.026$
$\mathrm{nrs}=0.026$ sa $=230.0 \mathrm{n}$ sb $=230.0 \mathrm{n}$ sca $=0$ scb $=0$ scc $=0$ mismatchflag $=1$ $\mathrm{nrs}=0.026$

M21 (net75 net75 vdd vdd) pch_mac $I=1 \mathrm{u} \quad \mathrm{w}=15.0 \mathrm{u}$ multi $=(4) \quad \mathrm{nf}=1$ ) $\mathrm{sd}=260.0 \mathrm{n}$ ad $=3.45 \mathrm{e}-12$ as $=3.45 \mathrm{e}-12 \mathrm{pd}=30.46 \mathrm{u} \mathrm{ps}=30.46 \mathrm{u}$ $\mathrm{nrd}=0.00866667 \mathrm{nrs}=0.00866667 \mathrm{sa}=230.0 \mathrm{n} \quad \mathrm{sb}=230.0 \mathrm{n}$ sca $=0 \quad \mathrm{scb}=0$ । $\mathrm{scC}=0$ mismatchflag $=1$ sigma $=1$

M19 (net043 net043 net75 net75) pch_mac $I=1 \mathrm{u} w=15.0 \mathrm{u}$ multi=(4) $n f=1$ । $\mathrm{sd}=260.0 \mathrm{n}$ ad $=3.45 \mathrm{e}-12$ as $=3.45 \mathrm{e}-12 \mathrm{pd}=30.46 \mathrm{u}$ ps $=30.46 \mathrm{u}$ $\mathrm{nrd}=0.00866667 \mathrm{nrs}=0.00866667 \mathrm{sa}=230.0 \mathrm{n} \quad \mathrm{sb}=230.0 \mathrm{n} \quad \mathrm{sca}=0 \quad \mathrm{scb}=0$ । $\mathrm{scC}=0$ mismatchflag $=1$ sigma $=1$

M18 (net0119 net043 vdd vdd) pch_mac $I=1 \mathrm{u} \mathrm{w}=15.0 \mathrm{u}$ multi=(4) $\mathrm{nf}=1$ ) $\mathrm{sd}=260.0 \mathrm{n}$ ad $=3.45 \mathrm{e}-12$ as $=3.45 \mathrm{e}-12 \mathrm{pd}=30.46 \mathrm{u}$ ps $=30.46 \mathrm{u}$ $\mathrm{nrd}=0.00866667 \mathrm{nrs}=0.00866667 \mathrm{sa}=230.0 \mathrm{n} \mathrm{sb}=230.0 \mathrm{n} \mathrm{sca}=0 \mathrm{scb}=0$ $\mathrm{scC}=0$ mismatchflag $=1$ sigma $=1$

M17 (net0120 net0105 vdd vdd) pch_mac $I=1 \mathrm{u} \quad \mathrm{w}=15.0 \mathrm{u}$ multi=(2) $\mathrm{nf}=1$ । $\mathrm{sd}=260.0 \mathrm{n}$ ad $=3.45 \mathrm{e}-12$ as $=3.45 \mathrm{e}-12 \mathrm{pd}=30.46 \mathrm{u} \mathrm{ps}=30.46 \mathrm{u}$ $\mathrm{nrd}=0.00866667 \mathrm{nrs}=0.00866667 \mathrm{sa}=230.0 \mathrm{n} \quad \mathrm{sb}=230.0 \mathrm{n} \quad \mathrm{sca}=0 \quad \mathrm{scb}=0$ । $\mathrm{nrd}=0.008667 \mathrm{hrs}=0.00866667$

M16 (net0105 net0105 net54 net54) pch_mac $I=1 \mathrm{u} w=15.0 \mathrm{u}$ multi=(4) $\mathrm{nf}=1$ । $\mathrm{sd}=260.0 \mathrm{n}$ ad $=3.45 \mathrm{e}-12$ as $=3.45 \mathrm{e}-12 \mathrm{pd}=30.46 \mathrm{u}$ ps $=30.46 \mathrm{u}$ $\mathrm{sd}=260.0 \mathrm{n} \mathrm{ad}=3.45 \mathrm{e}-12$ as $=3.45 \mathrm{e}-12 \mathrm{pd}=30.46 \mathrm{u} \mathrm{ps}=30.46 \mathrm{u}$
$\mathrm{nrd}=0.00866667 \mathrm{nrs}=0.00866667 \quad \mathrm{sa}=230.0 \mathrm{n} \quad \mathrm{sb}=230.0 \mathrm{n} \quad \mathrm{sca}=0 \quad \mathrm{scb}=0$ $\mathrm{nrd}=0.00866667 \mathrm{nrs}=0.0086666$

M4 (net092 net092 net19 net19) pch_mac $\mathrm{I}=1 \mathrm{u}$ w $=15.0 \mathrm{u}$ multi $=(4) \quad \mathrm{nf}=1$ ) $\mathrm{sd}=260.0 \mathrm{n}$ ad $=3.45 \mathrm{e}-12$ as $=3.45 \mathrm{e}-12 \mathrm{pd}=30.46 \mathrm{u}$ ps $=30.46 \mathrm{u}$ $\mathrm{nrd}=0.00866667 \mathrm{nrs}=0.00866667 \mathrm{sa}=230.0 \mathrm{n} \mathrm{sb}=230.0 \mathrm{n} \quad \mathrm{sca}=0 \quad \mathrm{scb}=0 \backslash$ 
$\mathrm{scC}=0$ mismatchflag $=1$ sigma $=1$

M9 (net42 net42 vdd vdd) pch_mac $\mathrm{I}=1 \mathrm{u} \quad \mathrm{w}=15.0 \mathrm{u}$ multi=(4) $\mathrm{nf}=1 \mathrm{sd}=260.0 \mathrm{n}$ ) $\mathrm{ad}=3.45 \mathrm{e}-12$ as $=3.45 \mathrm{e}-12 \mathrm{pd}=30.46 \mathrm{u}$ ps $=30.46 \mathrm{u} \mathrm{nrd}=0.00866667$ nrs $=0.00866667$ sa $=230.0$ n sb $=230.0$ n sca $=0 \quad s c b=0 \quad s c c=0$ । mismatchflag $=1$ sigma $=1$

M10 (net098 net098 net42 net42) pch mac $I=1 \mathrm{u} w=15.0 \mathrm{u}$ multi $=(4) \quad \mathrm{nf}=1$ $\mathrm{sd}=260.0 \mathrm{n}$ ad $=3.45 \mathrm{e}-12$ as $=3.45 \mathrm{e}-12 \mathrm{pd}=30.46 \mathrm{u}$ ps $=30.46 \mathrm{u}$ $\mathrm{sd}=260.0 \mathrm{n}$ ad $=3.45 \mathrm{e}-12 \mathrm{as}=3.45 \mathrm{e}-12 \mathrm{pd}=30.46 \mathrm{u} \mathrm{ps}=30.46 \mathrm{u}$
$\mathrm{nrd}=0.00866667 \mathrm{nrs}=0.00866667 \quad \mathrm{sa}=230.0 \mathrm{n} \quad \mathrm{sb}=230.0 \mathrm{n} \quad \mathrm{sca}=0 \quad \mathrm{scb}=0$ $\mathrm{nrd}=0.00866667$
$\mathrm{scc}=0$ mismatchflag $=1$ sigma $=1$

M15 (net54 net54 vdd vdd) pch_mac $\mathrm{I}=1 \mathrm{u} \quad \mathrm{w}=15.0 \mathrm{u}$ multi=(4) $\mathrm{nf}=1$ । $\mathrm{sd}=260.0 \mathrm{n}$ ad $=3.45 \mathrm{e}-12$ as $=3.45 \mathrm{e}-12 \mathrm{pd}=30.46 \mathrm{u}$ ps $=30.46 \mathrm{u}$ $\mathrm{nrd}=0.00866667 \mathrm{nrs}=0.00866667 \mathrm{sa}=230.0 \mathrm{n} \mathrm{sb}=230.0 \mathrm{n}$ sca $=0 \mathrm{scb}=0$ । $\mathrm{scc}=0$ mis matchflag $=1$ sigma $=1$

M11 (net0140 net098 vdd vdd) pch_mac I=1u $\mathrm{W}=15.0 \mathrm{u}$ multi=(2) $\mathrm{nf}=1$ ) $\mathrm{sd}=260.0 \mathrm{n}$ ad $=3.45 \mathrm{e}-12$ as $=3.45 \mathrm{e}-12 \mathrm{pd}=30.46 \mathrm{u} \mathrm{ps}=30.46 \mathrm{u}$ $\mathrm{nrd}=0.00866667 \mathrm{nrs}=0.00866667 \mathrm{sa}=230.0 \mathrm{n} \quad \mathrm{sb}=230.0 \mathrm{n} \quad \mathrm{sca}=0 \quad \mathrm{scb}=0$ । $\mathrm{scC}=0$ mismatchflag $=1$ sigma $=1$

M5 (net027 net092 vdd vdd) pch_mac $I=1 \mathrm{u} w=15.0 \mathrm{u}$ multi $=(4) \mathrm{nf}=1$ ) $\mathrm{sd}=260.0 \mathrm{n}$ ad $=3.45 \mathrm{e}-12$ as $=3.45 \mathrm{e}-12 \mathrm{pd}=30.46 \mathrm{u}$ ps $=30.46 \mathrm{u}$ $\mathrm{nrd}=0.00866667 \mathrm{nrs}=0.00866667 \mathrm{sa}=230.0 \mathrm{n} \quad \mathrm{sb}=230.0 \mathrm{n} \quad \mathrm{sca}=0 \quad \mathrm{scb}=0$ $\mathrm{scC}=0$ mismatchflag $=1$ sigma $=1$

M3 (net19 net19 vdd vdd) pch $\_$mac $I=1 \mathrm{u}$ w=15.0u multi=(4) $n f=1 \quad \mathrm{sd}=260.0 \mathrm{n}$ $\mathrm{ad}=3.45 \mathrm{e}-12$ as $=3.45 \mathrm{e}-12 \mathrm{pd}=30.46 \mathrm{u}$ ps $=30.46 \mathrm{u} \mathrm{nrd}=0.00866667$ $\mathrm{nrs}=0.00866667 \quad \mathrm{sa}=230.0 \mathrm{n} \mathrm{sb}=230.0 \mathrm{n} \quad \mathrm{sca}=0 \quad \mathrm{scb}=0 \quad \mathrm{scc}=0$ ends ineo $\begin{array}{ll}\mathrm{nrs}=0.00866667 & \mathrm{sa}=230.0 \\ \mathrm{mismatchflag}=1 & \text { sigma }=1\end{array}$

\section{E.2 Netlist do circuito bloco com 4 elementos de atrasso}

// Cell name: delay 4

subcht delay4 schematic

subckt delay4 in out vctrl vdd vss

M18 (out net038 vss vss) nch_mac $I=200 \mathrm{n} w=800 \mathrm{n}$ multi=(1) $\mathrm{nf}=1$ । $\mathrm{sd}=260.0 \mathrm{n}$ ad $=1.84 \mathrm{e}-13$ as $=1.84 \mathrm{e}-13 \mathrm{pd}=2.06 \mathrm{u}$ ps $=2.06 \mathrm{u} \mathrm{nrd}=0.1625$ $\mathrm{nrs}=0.1625 \mathrm{sa}=230.0 \mathrm{n} \mathrm{sb}=230.0 \mathrm{n}$ sca $=0 \mathrm{scb}=0 \mathrm{scc}=0$ mismatchflag $=1$ sigma $=1$

M15 (net038 net046 vss vss) nch_mac $\mathrm{I}=200 \mathrm{n} \mathrm{w}=400 \mathrm{n}$ multi $=(1) \mathrm{nf}=1$ > $\mathrm{sd}=260.0 \mathrm{n}$ ad $=9.2 \mathrm{e}-14$ as $=9.2 \mathrm{e}-14 \mathrm{pd}=1.26 \mathrm{u}$ ps $=1.26 \mathrm{u} \mathrm{nrd}=0.325$ $\mathrm{nrs}=0.325 \mathrm{sa}=230.0 \mathrm{n} \quad \mathrm{sb}=230.0 \mathrm{n} \quad \mathrm{sca}=0 \mathrm{scb}=0 \mathrm{scc}=0$ mismatchflag=1 sigma $=1$

M12 (net046 n5 vss vss) nch_mac $I=200 n \quad w=400 n$ multi $=(1) \quad n f=1 \quad s d=260.0 n$ ) $\mathrm{ad}=9.2 \mathrm{e}-14$ as $=9.2 \mathrm{e}-14 \mathrm{pd}=1.26 \mathrm{u} \mathrm{ps}=1.26 \mathrm{u} \mathrm{nrd}=0.325 \mathrm{nrs}=0.325$

11 (n5 n4 net044 vss) nch_mac $I=200 \mathrm{n} \quad \mathrm{w}=400 \mathrm{n}$ multi=(1) $\mathrm{nf}=1 \mathrm{sd}=260.0 \mathrm{n}$ $\mathrm{ad}=9.2 \mathrm{e}-14$ as $=9.2 \mathrm{e}-14 \mathrm{pd}=1.26 \mathrm{u}$ ps $=1.26 \mathrm{u} \mathrm{nrd}=0.325 \mathrm{nrs}=0.325$ $\mathrm{sa}=230.0 \mathrm{n} \quad \mathrm{sb}=230.0 \mathrm{n} \quad \mathrm{sca}=0 \quad \mathrm{scb}=0 \quad \mathrm{scc}=0$ mismatchflag $=1$ sigma $=1$

M10 (net044 vctrl vss vss) nch_mac $I=10 u \quad w=600 \mathrm{n}$ multi $=(1) \quad \mathrm{nf}=1$, $\mathrm{sd}=260.0 \mathrm{n}$ ad $=1.38 \mathrm{e}-13$ as $=1.38 \mathrm{e}-13 \mathrm{pd}=1.66 \mathrm{u} \mathrm{ps}=1.66 \mathrm{u} \mathrm{nrd}=0.216667$ $n r s=0.216667 \quad s a=230.0 n \quad s b=230.0 n \quad s c a=0 \quad s c b=0 \quad s c c=0$ mismatchflag $=1$ sigma $=1$

M9 (net024 vctrl vss vss) nch_mac $I=10 \mathrm{u} w=600 \mathrm{n}$ multi $=(1) \mathrm{nf}=1$ $\mathrm{sd}=260.0 \mathrm{n}$ ad $=1.38 \mathrm{e}-13$ as $=1.38 \mathrm{e}-13 \mathrm{pd}=1.66 \mathrm{u} \mathrm{ps}=1.66 \mathrm{u} \mathrm{nrd}=0.216667$ $\mathrm{nrs}=0.216667 \mathrm{sa}=230.0 \mathrm{n} \quad \mathrm{sb}=230.0 \mathrm{n} \quad \mathrm{sca}=0 \quad \mathrm{scb}=0 \quad \mathrm{scc}=0$ mismatchflag $=1$ sigma $=1$

M7 ( $n 4 \mathrm{n} 3$ vss vss) nch_mac $\mathrm{I}=200 \mathrm{n} \mathrm{w}=400 \mathrm{n}$ multi $=(1) \mathrm{nf}=1 \mathrm{sd}=260.0 \mathrm{n}$ ) $\mathrm{ad}=9.2 \mathrm{e}-14$ as $=9.2 \mathrm{e}-14 \mathrm{pd}=1.26 \mathrm{u} \mathrm{ps}=1.26 \mathrm{u} \mathrm{nrd}=0.325 \mathrm{nrs}=0.325$ $\mathrm{sa}=230.0 \mathrm{n} \quad \mathrm{sb}=230.0 \mathrm{n} \quad \mathrm{sca}=0 \quad \mathrm{scb}=0 \quad \mathrm{scc}=0$ mismatchflag $=1$ sigma $=1$

M5 (n3 n2 net024 vss) nch_mac $I=200 n \quad w=400 n$ multi $=(1)$ nf $=1$ sd $=260$.0n $\mathrm{ad}=9.2 \mathrm{e}-14$ as $=9.2 \mathrm{e}-14 \mathrm{pd}=1.26 \mathrm{u} \mathrm{ps}=1.26 \mathrm{u} \mathrm{nrd}=0.325 \mathrm{nrs}=0.325$

MO ( $\mathrm{n} 1$ in net18 vss) nch 1 mac $I=200 \mathrm{n} \quad \mathrm{w}=400 \mathrm{n}$ multi $=(1) \mathrm{nf}=1 \quad \mathrm{sd}=260.0 \mathrm{n}$ $\mathrm{ad}=9.2 \mathrm{e}-14$ as $=9.2 \mathrm{e}-14 \mathrm{pd}=1.26 \mathrm{u}$ ps $=1.26 \mathrm{u} \mathrm{nrd}=0.325 \mathrm{nrs}=0.325$ $\mathrm{sa}=230.0 \mathrm{n} \quad \mathrm{sb}=230.0 \mathrm{n} \quad \mathrm{sca}=0 \quad \mathrm{scb}=0 \quad \mathrm{scc}=0$ mismatchflag $=1$ sigma $=1$

M4 (n2 n1 vss vss) nch_mac $I=200 n \quad w=400 n$ multi $=(1) \quad n f=1 \quad s d=260.0 n$ । $\mathrm{ad}=9.2 \mathrm{e}-14$ as $=9.2 \mathrm{e}-14 \mathrm{pd}=1.26 \mathrm{u}$ ps $=1.26 \mathrm{u} \mathrm{nrd}=0.325 \mathrm{nrs}=0.325$ $\mathrm{sa}=230.0 \mathrm{n} \quad \mathrm{sb}=230.0 \mathrm{n} \quad \mathrm{sca}=0 \quad \mathrm{scb}=0 \quad \mathrm{scc}=0$ mismatchflag $=1$ sigma $=1$

M1 (net18 vctrl vss vss) nch_mac $I=10 u \quad w=600 \mathrm{n}$ multi $=$ (1) $\mathrm{nf}=1 \mathrm{sd}=260.0 \mathrm{n}$ $\mathrm{ad}=1.38 \mathrm{e}-13$ as $=1.38 \mathrm{e}-13 \mathrm{pd}=1.66 \mathrm{u}$ ps $=1.66 \mathrm{u}$ nrd $=0.216667$ । $\mathrm{nrs}=0.216667 \mathrm{sa}=230.0 \mathrm{n} \mathrm{sb}=230.0 \mathrm{n}$ sca $=0 \quad \mathrm{scb}=0 \quad \mathrm{scc}=0$ mismatchflag $=1$ sigma $=1$

M17 (out net038 vdd vdd) pch_mac $I=200 n \quad w=1.6 u$ multi=(1) $n f=1$ ) $\mathrm{sd}=260.0 \mathrm{n} \mathrm{ad}=3.68 \mathrm{e}-13$ as $=3.68 \mathrm{e}-13 \mathrm{pd}=3.66 \mathrm{u} \mathrm{ps}=3.66 \mathrm{u} \mathrm{nrd}=0.08125$ । $n r s=0.08125 \mathrm{sa}=230.0 \mathrm{n}$ sb=230.0n $\mathrm{sca}=0 \mathrm{scb}=0 \mathrm{scc}=0$ mismatchflag $=1$ sigma $=1$

M16 ( $\mathrm{sd}=260.0 \mathrm{n}$ ad $=1.84 \mathrm{e}-13$ as $=1.84 \mathrm{e}-13 \mathrm{pd}=2.06 \mathrm{u} \mathrm{ps}=2.06 \mathrm{u}$ nrd $=0.1625$ $\mathrm{nrs}=0.1625 \mathrm{sa}=230.0 \mathrm{n} \mathrm{sb}=230.0 \mathrm{n} \quad \mathrm{sca}=0 \mathrm{scb}=0 \quad \mathrm{scc}=0$ mismatchflag $=1$ sigma $=1$

M14 ( net046 n5 vdd vdd) pch_mac $I=200 \mathrm{n} w=800 \mathrm{n}$ multi=(1) $\mathrm{nf}=1 \mathrm{sd}=260.0 \mathrm{n}$ $\mathrm{ad}=1.84 \mathrm{e}-13$ as $=1.84 \mathrm{e}-13 \mathrm{pd}=2.06 \mathrm{u}$ ps $=2.06 \mathrm{u} \mathrm{nrd}=0.1625 \mathrm{nrs}=0.1625$, $\mathrm{sa}=230.0 \mathrm{n} \quad \mathrm{sb}=230$. On $\mathrm{sca}=0 \quad \mathrm{scb}=0 \quad \mathrm{scc}=0$ mismatchflag $=1$ sigma $=1$

M13 ( $\mathrm{n} 5 \mathrm{n} 4$ vdd vdd) pch_mac $\mathrm{I}=200 \mathrm{n} \mathrm{w}=800 \mathrm{n}$ multi=(1) $\mathrm{nf}=1 \mathrm{sd}=260.0 \mathrm{n}$ $\mathrm{ad}=1.84 \mathrm{e}-13$ as $=1.84 \mathrm{e}-13 \mathrm{pd}=2.06 \mathrm{u}$ ps $=2.06 \mathrm{u} \mathrm{nrd}=0.1625 \mathrm{nrs}=0.1625$ । $\mathrm{sa}=230.0 \mathrm{n} \quad \mathrm{sb}=230$. 0 n $\mathrm{sca}=0 \quad \mathrm{scb}=0 \quad \mathrm{scc}=0$ mismatchflag $=1$ sigma $=1$

M8 (n4 n3 vdd vdd) pch_mac $I=200 n \quad w=800 n$ multi=(1) $n f=1 \quad s d=260$.0n $\mathrm{ad}=1.84 \mathrm{e}-13$ as $=1.84 \mathrm{e}-13 \mathrm{pd}=2.06 \mathrm{u}$ ps $=2.06 \mathrm{u}$ nrd $=0.1625 \mathrm{nrs}=0.1625$ । $\mathrm{sa}=230.0 \mathrm{n} \quad \mathrm{sb}=230.0 \mathrm{n} \mathrm{sca}=0 \mathrm{scb}=0 \mathrm{scc}=0$ mismatchflag $=1$ sigma $=1$

M6 (n3 n2 vdd vdd) pch_mac $I=200 n \quad w=800 n$ multi $=(1) \quad n f=1 \quad s d=260$.0n $\mathrm{ad}=1.84 \mathrm{e}-13 \mathrm{as}=1.84 \mathrm{e}-13 \mathrm{pd}=2.06 \mathrm{u}$ ps $=2.06 \mathrm{u} \mathrm{nrd}=0.1625 \mathrm{nrs}=0.1625$ $\mathrm{sa}=230 . \mathrm{n} \quad \mathrm{sb}=230 . \mathrm{n} \mathrm{sca}=0 \mathrm{scb}=0 \mathrm{scc}=0$ mismatchflag $=1$ sigma $=1$

M3 (n2 n1 vdd vdd) pch_mac $I=200 n w=800 n$ multi=(1) $n f=1 \quad s d=260$.0n 
$\mathrm{ad}=1.84 \mathrm{e}-13 \mathrm{as}=1.84 \mathrm{e}-13 \mathrm{pd}=2.06 \mathrm{u}$ ps $=2.06 \mathrm{u} \mathrm{nrd}=0.1625 \mathrm{nrs}=0.1625$ $\mathrm{sa}=230.0 \mathrm{n} \quad \mathrm{sb}=230.0 \mathrm{n} \quad \mathrm{sca}=0 \mathrm{scb}=0 \mathrm{scc}=0$ mismatchflag $=1$ sigma $=1$

M2 ( $\mathrm{n} 1$ in vdd vdd) pch mac $\mathrm{I}=200 \mathrm{n} \mathrm{w}=800 \mathrm{n}$ multi $=(1) \mathrm{nf}=1 \mathrm{sd}=260$. On

$\mathrm{ad}=1.84 \mathrm{e}-13$ as $=1.84 \mathrm{e}-13 \mathrm{pd}=2.06 \mathrm{u} \mathrm{ps}=2.06 \mathrm{u} \mathrm{nrd}=0.1625 \mathrm{nrs}=0.1625$ ends delay 4

$\mathrm{sa}=230.0 \mathrm{n} \quad \mathrm{sb}=230.0 \mathrm{n} \quad \mathrm{sca}=0 \quad \mathrm{scb}=0 \quad \mathrm{scc}=0$ mismatchflag $=1$ sigma $=1$

\section{E.3 Netlist da simulação do calculo de distância}

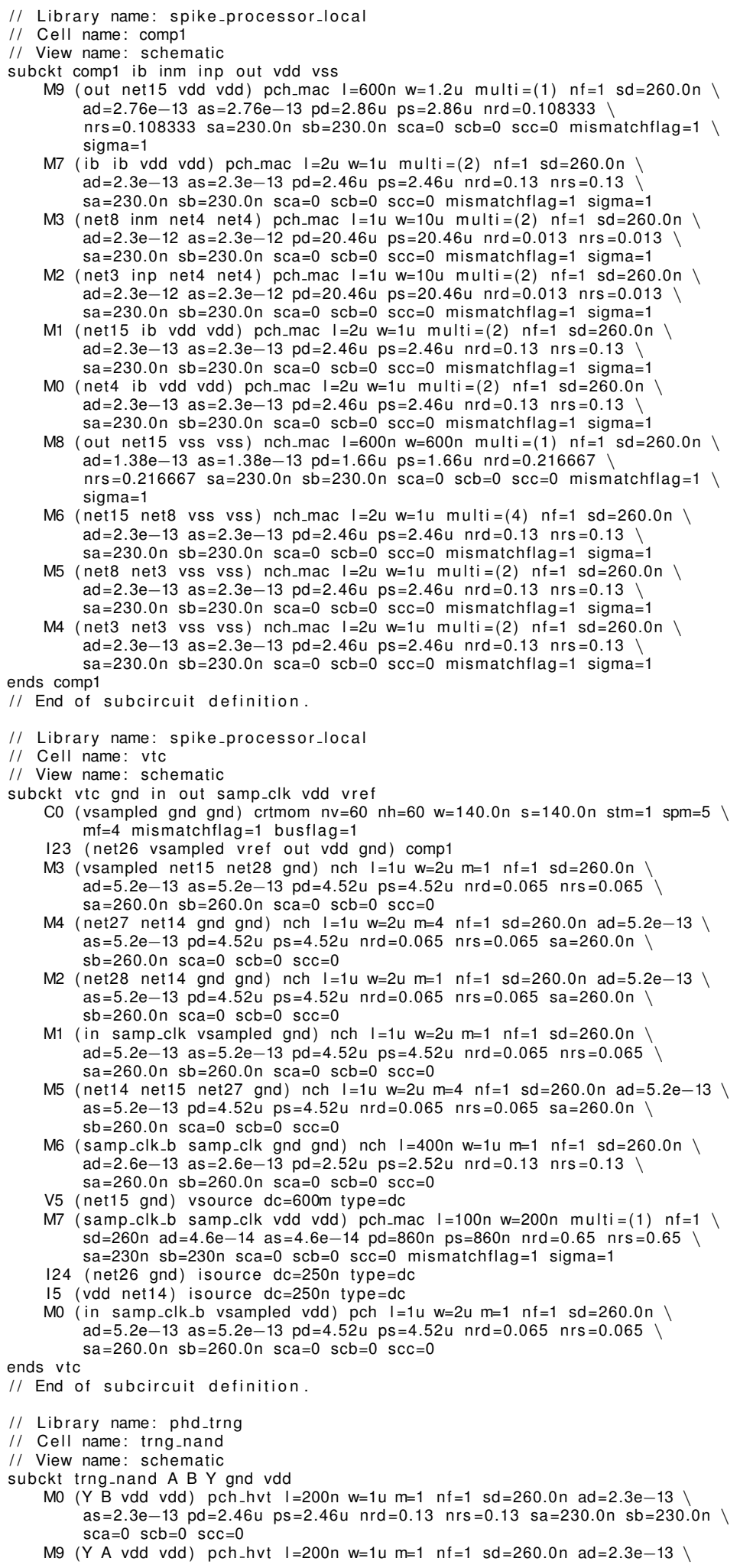


as $=2.3 \mathrm{e}-13 \mathrm{pd}=2.46 \mathrm{u} \mathrm{ps}=2.46 \mathrm{u} \mathrm{nrd}=0.13 \mathrm{nrs}=0.13 \mathrm{sa}=230.0 \mathrm{n} \quad \mathrm{sb}=230.0 \mathrm{n} \backslash$ $\mathrm{sca}=0 \quad \mathrm{scb}=0 \quad \mathrm{scc}=0$

M1 (net30 A gnd gnd) nch_hvt $\mathrm{I}=200 \mathrm{n} \mathrm{w}=500 \mathrm{n} \mathrm{m}=1 \mathrm{nf}=1 \mathrm{sd}=260.0 \mathrm{n}$ $\mathrm{ad}=1.15 \mathrm{e}-13 \mathrm{as}=1.15 \mathrm{e}-13 \mathrm{pd}=1.46 \mathrm{u}$ ps $=1.46 \mathrm{u} \mathrm{nrd}=0.26 \mathrm{nrs}=0.26$ । $\mathrm{sa}=230.0 \mathrm{n} \quad \mathrm{sb}=230$. 0 n $\mathrm{sca}=0 \quad \mathrm{scb}=0 \quad \mathrm{scc}=0$

M8 ( $Y$ B net30 gnd) nch hvt $I=200 n \quad w=500 n \quad m=1 \quad n f=1 \quad s d=260.0 n$ $\mathrm{ad}=1.15 \mathrm{e}-13$ as $=1.15 \mathrm{e}-13 \mathrm{pd}=1.46 \mathrm{u} \mathrm{ps}=1.46 \mathrm{u} \mathrm{nrd}=0.26 \mathrm{nrs}=0.26$ । $\mathrm{ad}=1.15 \mathrm{e}-13$ as $=1.15 \mathrm{e}-13$ pd $=1.46 \mathrm{u} \quad \mathrm{ps}=1$
$\mathrm{sa}=230.0 \mathrm{n}$ sb $=230.0$ n $\mathrm{sca}=0 \quad \mathrm{scb}=0 \quad \mathrm{scc}=0$

ends tring_nand

// End of subcircuit definition.

// Library name: spike_processor_local

// Cell name: delay4

// View name: schematic

subckt delay4 in out vctrl vdd vss

M18 (out net038 vss vss) nch_mac $I=200 n \quad w=800 n$ multi $=(1) \quad n f=1$ $\mathrm{sd}=260.0 \mathrm{n}$ ad $=1.84 \mathrm{e}-13$ as $=1.84 \mathrm{e}-13 \mathrm{pd}=2.06 \mathrm{u} \mathrm{ps}=2.06 \mathrm{u} \mathrm{nrd}=0.1625$ $\mathrm{nrs}=0.1625 \mathrm{sa}=230.0 \mathrm{n} \mathrm{sb}=230.0 \mathrm{n}$ sca $=0 \mathrm{scb}=0 \mathrm{scc}=0$ mismatchflag=1 sigma $=1$

M15 (net038 net046 vss vss) nch_mac $I=200 n \mathrm{w}=400 \mathrm{n}$ multi $=(1) \mathrm{nf}=1$ $\mathrm{sd}=260.0 \mathrm{n}$ ad $=9.2 \mathrm{e}-14$ as $=9.2 \mathrm{e}-14 \mathrm{pd}=1.26 \mathrm{u} \mathrm{ps}=1.26 \mathrm{u} \mathrm{nrd}=0.325$ $\mathrm{nrs}=0.325 \mathrm{sa}=230.0 \mathrm{n} \quad \mathrm{sb}=230.0 \mathrm{n} \quad \mathrm{sca}=0 \quad \mathrm{scb}=0 \quad \mathrm{scc}=0$ mismatchflag $=1$ $\mathrm{nrs}=0.325$
sigma $=1$

M12 (net046 n5 vss vss) nch mac $I=200 \mathrm{n} w=400 \mathrm{n}$ multi=(1) $\mathrm{nf}=1 \mathrm{sd}=260.0 \mathrm{n}$ \ $\mathrm{ad}=9.2 \mathrm{e}-14$ as $=9.2 \mathrm{e}-14 \mathrm{pd}=1.26 \mathrm{u}$ ps $=1.26 \mathrm{u}$ nrd $=0.325 \mathrm{nrs}=0.325$ $\mathrm{sa}=230.0 \mathrm{n} \quad \mathrm{sb}=230.0 \mathrm{n} \quad \mathrm{sca}=0 \quad \mathrm{scb}=0 \quad \mathrm{scc}=0$ mismatchflag $=1$ sigma $=1$

M11 (n5 n4 net044 vss) nch_mac $I=200 \mathrm{n} w=400 \mathrm{n}$ multi=(1) $\mathrm{nf}=1$ sd=260.0n $\mathrm{ad}=9.2 \mathrm{e}-14 \mathrm{as}=9.2 \mathrm{e}-14 \mathrm{pd}=1.26 \mathrm{u} \mathrm{ps}=1.26 \mathrm{u} \mathrm{nrd}=0.325 \mathrm{nrs}=0.325$ $\mathrm{sa}=230.0 \mathrm{n} \quad \mathrm{sb}=230.0 \mathrm{n} \quad \mathrm{sca}=0 \quad \mathrm{scb}=0 \quad \mathrm{scc}=0$ mismatchflag $=1$ sigma $=1$

M10 (net044 vctrl vss vss) nch_mac $\mathrm{I}=10 \mathrm{u} \mathrm{w}=600 \mathrm{n}$ multi $=(1) \mathrm{nf}=1$ ) $\mathrm{sd}=260.0 \mathrm{n} \mathrm{ad}=1.38 \mathrm{e}-13$ as $=1.38 \mathrm{e}-13 \mathrm{pd}=1.66 \mathrm{u}$ ps $=1.66 \mathrm{u}$ nrd $=0.216667$ $\mathrm{nrs}=0.216667 \mathrm{sa}=230.0 \mathrm{n} \quad \mathrm{sb}=230.0 \mathrm{n} \quad \mathrm{sca}=0 \quad \mathrm{scb}=0 \quad \mathrm{scc}=0$ mismatchflag $=1$ sigma $=1$

M9 (net024 vctrl vss vss) nch_mac $\mathrm{I}=10 \mathrm{u} w=600 \mathrm{n}$ multi $=(1) \mathrm{nf}=1$ ) $\mathrm{sd}=260.0 \mathrm{n}$ ad $=1.38 \mathrm{e}-13$ as $=1.38 \mathrm{e}-13 \mathrm{pd}=1.66 \mathrm{u} \mathrm{ps}=1.66 \mathrm{u} \mathrm{nrd}=0.216667$ $\mathrm{nrs}=0.216667 \mathrm{sa}=230.0 \mathrm{n} \mathrm{sb}=230.0 \mathrm{n}$ sca $=0 \mathrm{scb}=0 \mathrm{scc}=0$ mismatchflag=1 \} sigma $=1$

M7 (n4 n3 vss vss) nch_mac $I=200 n \quad w=400 n$ multi $=(1) n f=1 \quad s d=260.0 n$ ) $\mathrm{ad}=9.2 \mathrm{e}-14$ as $=9.2 \mathrm{e}-14 \mathrm{pd}=1.26 \mathrm{u}$ ps $=1.26 \mathrm{u} \mathrm{nrd}=0.325 \mathrm{nrs}=0.325$ $\mathrm{ad}=9.2 \mathrm{e}-14$ as $=9.2 \mathrm{e}-14 \mathrm{pd}=1.26 \mathrm{u}$ ps $=1.26 \mathrm{u}$ nrd $=0.325 \mathrm{nrs}=0.325$

M5 (n3 n2 net024 vss) nch_mac $I=200 n \quad w=400 n$ multi=(1) $n f=1 \quad s d=260.0 n$ $\mathrm{ad}=9.2 \mathrm{e}-14$ as $=9.2 \mathrm{e}-14 \mathrm{pd}=1.26 \mathrm{u}$ ps $=1.26 \mathrm{u}$ nrd $=0.325 \mathrm{nrs}=0.325$ $\mathrm{ad}=9.2 \mathrm{e}-14$ as $=9.2 \mathrm{e}-14 \quad \mathrm{pd}=1.26 \mathrm{u} \mathrm{ps}=1.26 \mathrm{u} \mathrm{nrd}=0.325 \mathrm{nrs}=0.325$
$\mathrm{sa}=230.0 \mathrm{n} \quad \mathrm{sb}=230.0 \mathrm{n} \quad \mathrm{sca}=0 \quad \mathrm{scb}=0 \quad \mathrm{scc}=0$ mismatchflag $=1$ sigma $=1$

MO ( $\mathrm{n} 1$ in net18 vss) nch_mac $\mathrm{I}=200 \mathrm{n} \quad \mathrm{w}=400 \mathrm{n}$ multi $=(1) \mathrm{nf}=1 \quad \mathrm{sd}=260.0 \mathrm{n}$ $\mathrm{ad}=9.2 \mathrm{e}-14$ as $=9.2 \mathrm{e}-14 \mathrm{pd}=1.26 \mathrm{u}$ ps $=1.26 \mathrm{u} \mathrm{nrd}=0.325 \mathrm{nrs}=0.325$ $\mathrm{sa}=230.0 \mathrm{n} \mathrm{sb}=230.0 \mathrm{n} \quad \mathrm{sca}=0 \quad \mathrm{scb}=0 \quad \mathrm{scc}=0$ mismatchflag $=1$ sigma $=1$

M4 (n2 n1 vss vss) nch_mac $I=200 n \mathrm{w}=400 \mathrm{n}$ multi $=(1) \mathrm{nf}=1 \mathrm{sd}=260.0 \mathrm{n}$ । $\mathrm{ad}=9.2 \mathrm{e}-14$ as $=9.2 \mathrm{e}-14 \mathrm{pd}=1.26 \mathrm{u}$ ps $=1.26 \mathrm{u} \mathrm{nrd}=0.325 \mathrm{nrs}=0.325$ $\mathrm{sa}=230.0 \mathrm{n} \quad \mathrm{sb}=230.0 \mathrm{n}$ sca $=0 \quad \mathrm{scb}=0 \quad \mathrm{scc}=0$ mismatchflag $=1$ sigma $=1$

M1 (net18 vctrl vss vss) nch_mac $I=10 u \quad w=600 n$ multi $=(1) \quad n f=1 \quad s d=260.0 n$ $\mathrm{ad}=1.38 \mathrm{e}-13$ as $=1.38 \mathrm{e}-13 \mathrm{pd}=1.66 \mathrm{u} \mathrm{ps}=1.66 \mathrm{u} \mathrm{nrd}=0.216667$ $\mathrm{nrs}=0.216667 \mathrm{sa}=230.0 \mathrm{n} \mathrm{sb}=230.0 \mathrm{n} \mathrm{sca}=0 \mathrm{scb}=0 \mathrm{scc}=0$ mismatchflag=1 । sigma $=1$

M17 ( out net038 vdd vdd) pch_mac $I=200 \mathrm{n} w=1.6 \mathrm{u}$ multi=(1) $n f=1$ । $\mathrm{sd}=260.0 \mathrm{n}$ ad $=3.68 \mathrm{e}-13 \mathrm{as}=3.68 \mathrm{e}-13 \mathrm{pd}=3.66 \mathrm{u}$ ps $=3.66 \mathrm{u} \mathrm{nrd}=0.08125$ । $\mathrm{nrs}=0.08125$ sa $=230.0 \mathrm{n}$ sb $=230.0 \mathrm{n} \quad \mathrm{sca}=0 \quad \mathrm{scb}=0 \quad \mathrm{scc}=0$ mismatchflag $=1$ sigma $=1$

M16 (net038 net046 vdd vdd) pch_mac $I=200 \mathrm{n} w=800 \mathrm{n}$ multi $=(1) \mathrm{nf}=1$ $\mathrm{sd}=260.0 \mathrm{n}$ ad $=1.84 \mathrm{e}-13$ as $=1.84 \mathrm{e}-13 \mathrm{pd}=2.06 \mathrm{u}$ ps $=2.06 \mathrm{u}$ nrd $=0.1625$ $\mathrm{nrs}=0.1625 \mathrm{sa}=230.0 \mathrm{n} \mathrm{sb}=230.0 \mathrm{n}$ sca $=0 \quad \mathrm{scb}=0 \quad \mathrm{scc}=0$ mismatchflag $=1$ । sigma $=1$

M14 (net046 n5 vdd vdd) pch_mac $I=200 n \quad w=800 n$ multi=(1) $n f=1 \quad s d=260.0 n$ $\mathrm{ad}=1.84 \mathrm{e}-13$ as $=1.84 \mathrm{e}-13 \mathrm{pd}=2.06 \mathrm{u}$ ps $=2.06 \mathrm{u} \mathrm{nrd}=0.1625 \mathrm{nrs}=0.1625$ । $\mathrm{sa}=230.0 \mathrm{n} \quad \mathrm{sb}=230.0 \mathrm{n} \quad \mathrm{sca}=0 \quad \mathrm{scb}=0 \quad \mathrm{scc}=0$ mismatchflag $=1$ sigma $=1$

M13 (n5 n4 vdd vdd) pch_mac $I=200 n \quad w=800 n$ multi=(1) $n f=1 \quad s d=260$.0n $\mathrm{ad}=1.84 \mathrm{e}-13 \mathrm{as}=1.84 \mathrm{e}-13 \mathrm{pd}=2.06 \mathrm{u}$ ps $=2.06 \mathrm{u} \mathrm{nrd}=0.1625 \mathrm{nrs}=0.1625$ $\mathrm{sa}=230.0 \mathrm{n} \quad \mathrm{sb}=230.0 \mathrm{n} \quad \mathrm{sca}=0 \quad \mathrm{scb}=0 \quad \mathrm{scc}=0$ mismatchflag $=1$ sigma $=1$

M8 (n4 n3 vdd vdd) pch_mac $I=200 n \quad w=800 n$ multi=(1) $n f=1 \quad s d=260$. On $\mathrm{ad}=1.84 \mathrm{e}-13 \mathrm{as}=1.84 \mathrm{e}-13 \mathrm{pd}=2.06 \mathrm{u}$ ps $=2.06 \mathrm{u} \mathrm{nrd}=0.1625 \mathrm{nrs}=0.1625$ ) $\mathrm{sa}=230.0 \mathrm{n} \quad \mathrm{sb}=230.0 \mathrm{n} \quad \mathrm{sca}=0 \quad \mathrm{scb}=0 \quad \mathrm{scc}=0$ mismatchflag $=1$ sigma $=1$

M6 (n3 n2 vdd vdd) pch_mac I=200n $w=800 n$ multi $=(1) \quad n f=1 \quad s d=260$. 0 n $\mathrm{ad}=1.84 \mathrm{e}-13$ as $=1.84 \mathrm{e}-13 \quad \mathrm{pd}=2.06 \mathrm{u} \quad \mathrm{ps}=2.06 \mathrm{u}$ nrd $=0.1625$ nrs $=0.1625$
$\mathrm{sa}=230.0 \mathrm{n} \quad \mathrm{sb}=230.0 \mathrm{n}$ sca $=0 \quad \mathrm{scb}=0 \quad \mathrm{scc}=0$ mismatchflag $=1$ sigma $=1$

13 (n2 1 . $\mathrm{ad}=1.84 \mathrm{e}-13$ as $=1.84 \mathrm{e}-13 \mathrm{pd}=2.06 \mathrm{u} \mathrm{ps}=2.06 \mathrm{u} \mathrm{nrd}=0.1625 \mathrm{nrs}=0.1625$ । $\mathrm{ad}=1.84 \mathrm{e}-13$ as $=1.84 \mathrm{e}-13 \mathrm{pd}=2.06 \mathrm{ps}=2.06 \mathrm{n}$ m $\mathrm{sb}=230.0 \mathrm{n} \quad \mathrm{sca}=0 \quad \mathrm{scb}=0 \quad \mathrm{scc}=0$ mismatchflag $=1$ sigma $=1$

M2 ( $\mathrm{n} 1$ in vdd vdd) pch_mac $\quad I=200 \mathrm{n} \quad \mathrm{w}=800 \mathrm{n}$ multi=(1) $\mathrm{nf}=1 \quad \mathrm{sd}=260.0 \mathrm{n}$ $\mathrm{ad}=1.84 \mathrm{e}-13$ as $=1.84 \mathrm{e}-13 \mathrm{pd}=2.06 \mathrm{u} \mathrm{ps}=2.06 \mathrm{u} \mathrm{nrd}=0.1625 \mathrm{nrs}=0.1625$ । $\mathrm{sa}=230.0 \mathrm{n}$ sb $=230.0 \mathrm{n}$ sca $=0 \quad \mathrm{scb}=0 \quad \mathrm{scc}=0$ mismatchflag $=1$ sigma $=1$ ends delay 4

// End of subcircuit definition.

// Library name: phd_trng

// Cell name: manhattan_xor

// View name: schematic

subckt manhattan_xor A B Y gnd vdd

M6 ( $Y$ net019 vdd vdd) pch_hvt $\mathrm{l}=1 \mathrm{u} \quad \mathrm{w}=2 \mathrm{u} \quad \mathrm{m}=1 \quad \mathrm{nf}=1 \quad \mathrm{sd}=260.0 \mathrm{n}$ ad=5.2e-13 as $=5.2 \mathrm{e}-13 \mathrm{pd}=4.52 \mathrm{u} \mathrm{ps}=4.52 \mathrm{u} \mathrm{nrd}=0.065 \mathrm{nrs}=0.065 \mathrm{sa}=260.0 \mathrm{n}$ $\mathrm{sb}=260.0 \mathrm{n} \quad \mathrm{sca}=0 \quad \mathrm{scb}=0 \quad \mathrm{scc}=0$

M4 (net019 B net017 vdd) pch_hvt $\mathrm{I}=1 \mathrm{u} w=2 \mathrm{u} \mathrm{m}=1 \mathrm{nf}=1 \mathrm{sd}=260.0 \mathrm{n}$ $\mathrm{ad}=5.2 \mathrm{e}-13$ as $=5.2 \mathrm{e}-13 \mathrm{pd}=4.52 \mathrm{u} \mathrm{ps}=4.52 \mathrm{u} \mathrm{nrd}=0.065 \mathrm{nrs}=0.065$ $\mathrm{ad}=5.2 \mathrm{e}-13$ as $=5.2 \mathrm{e}-13$ pd $=4.52 \mathrm{u}$ ps $=4.52$
$\mathrm{sa}=260.0 \mathrm{n} \quad \mathrm{sb}=260.0 \mathrm{n} \quad \mathrm{sca}=0 \quad \mathrm{scb}=0 \quad \mathrm{scc}=0$

M3 (net017 A vdd vdd) pch _hvt $I=1 \mathrm{u}^{\mathrm{w}} \mathrm{w}=2 \mathrm{u} \quad \mathrm{m}=1 \quad \mathrm{nf}=1 \mathrm{sd}=260.0 \mathrm{n}$ ad=5.2e-13 as $=5.2 \mathrm{e}-13 \mathrm{pd}=4.52 \mathrm{u}$ ps $=4.52 \mathrm{u}$ nrd $=0.065 \mathrm{nrs}=0.065 \mathrm{sa}=260.0 \mathrm{n}$ $\mathrm{sb}=260.0 \mathrm{n} \quad \mathrm{sca}=0 \quad \mathrm{scb}=0 \quad \mathrm{scc}=0$

M7 ( $\mathrm{Y}$ net019 gnd gnd) nch_hvt $\mathrm{I}=1 \mathrm{u} \quad \mathrm{w}=1 \mathrm{u} \mathrm{m}=1 \quad \mathrm{nf}=1 \mathrm{sd}=260.0 \mathrm{n}$ ad=2.6e-13 as $=2.6 \mathrm{e}-13 \mathrm{pd}=2.52 \mathrm{u}$ ps $=2.52 \mathrm{u} \mathrm{nrd}=0.13 \mathrm{nrs}=0.13 \mathrm{sa}=260.0 \mathrm{n} \quad \mathrm{sb}=260.0 \mathrm{n}$ $\mathrm{sca}=0 \quad \mathrm{scb}=0 \quad \mathrm{scc}=0$ 
M5 (net019 B A gnd) nch_hvt $\mathrm{I}=1 \mathrm{u} \quad \mathrm{w}=1 \mathrm{u} \mathrm{m}=1 \quad \mathrm{nf}=1 \quad \mathrm{sd}=260.0 \mathrm{n}$ ad=2.6e-13 as $=2.6 \mathrm{e}-13 \mathrm{pd}=2.52 \mathrm{u}$ ps=2.52u $\mathrm{nrd}=0.13 \mathrm{nrs}=0.13 \mathrm{sa}=260.0 \mathrm{n} \quad \mathrm{sb}=260.0 \mathrm{n} \backslash$ $\mathrm{sca}=0 \quad \mathrm{scb}=0 \quad \mathrm{scc}=0$

M2 (net017 A gnd gnd) nch - hvt $\mathrm{I}=1 \mathrm{u} \quad \mathrm{w}=1 \mathrm{u} \mathrm{m}=1 \mathrm{nf}=1 \mathrm{sd}=260.0 \mathrm{n}$ ad=2.6e-13 as $=2.6 \mathrm{e}-13 \mathrm{pd}=2.52 \mathrm{u}$ ps $=2.52 \mathrm{u} \mathrm{nrd}=0.13 \mathrm{nrs}=0.13 \mathrm{sa}=260.0 \mathrm{n} \quad \mathrm{sb}=260.0 \mathrm{n}$ as $=2.6 \mathrm{e}-13$ pd $=2.52$
$\mathrm{sca}=0 \quad \mathrm{scb}=0 \quad \mathrm{scc}=0$

ends manhattan xor

$/ /$ End of subcircuit definition.

// Library name: spike_processor_local

/l Cell name: sim_distance_single_source

// View name: schematic

$|106 \backslash<1|>$ (vss vin $\backslash<1 \backslash>$ pulse_in $\backslash<1\rangle>$ samp_clk vdd vref) vtc

$|106|<2 \mid>$ (vss vin $|<2|>$ pulse_in $|<2|>$ samp_clk vdd vref) vtc

$|106 \backslash<3|>$ (vss vin $\backslash<3 \mid>$ pulse_in $\backslash<3 \backslash>$ samp_clk vdd vref) vtc

$|106|<4 \mid>$ (vss vin $\backslash<4 \mid>$ pulse_in $|<4|>$ samp_clk vdd vref) vtc

$|106|<5 \backslash>$ (vss vin $\backslash<5 \backslash>$ pulse_in $\mid<5 \backslash>$ samp_clk vdd vref) vtc

$|106|<6 \mid>$ (vss vin $\backslash<6 \mid>$ pulse_in $|<6|>$ samp_clk vdd vref) vtc

$|106|<7\rangle>$ (vss vin $\backslash<7\rangle>$ pulse_in $|<7\rangle>$ samp_clk vdd vref) vtc

$\mid 106 \backslash<8 \backslash>$ (vss vin $\backslash<8 \backslash>$ pulse_in $\backslash<8 \backslash>$ samp_clk vdd vref) vtc

$|106|<9\rangle>$ (vss vin $\backslash<9\rangle>$ pulse in $|<9|>$ samp_clk vdd vref) vtc

$\mid 106 \backslash<10 \backslash>$ (vss vin $\backslash<10 \backslash>$ pulse_in $\backslash<10 \backslash>$ samp_clk vdd vref) vtc

$|106|<11 \backslash>$ (vss vin $\langle<11 \backslash>$ pulse_in $\mid<11\rangle>$ samp_clk vdd vref) vtc

$1106<<12>$ (vss vin $<12 \mid>$ puls. in $<12<$ samp_clk vdd vref) vtc

$1106\langle<13\rangle$ (vss vin $\langle 13\rangle$ puls $<1<13\rangle$ samp_clk vdd vref) vtc

$100<<13<>$ (vss vin $<13<>$ pulse_in $\langle<13<>$ samp_clk vdd vref) vtc

$1106<<14 \mid>$ (vss vin $\langle<14|>$ pulse_in $\langle<14|>$ samp_clk vdd vref) vtc

$1106 \backslash<15 \backslash>$ (vss vin $\backslash<15 \backslash>$ pulse_in $\backslash<15 \backslash>$ samp_clk vdd vref) vtc

$\mid 106 \backslash<16 \backslash>$ (vss vin $\backslash<16 \backslash>$ pulse_in $\backslash<16 \backslash>$ samp_clk vdd vref) vtc

$1106 \backslash<17 \backslash>$ (vss vin $\backslash<17 \backslash>$ pulse_in $\backslash<17 \backslash>$ samp_clk vdd vref) vtc

$|106|<18 \mid>$ (vss vin $|<18|>$ pulse_in $|<18|>$ samp_clk vdd vref) vtc

$|106|<19 \mid>$ (vss vin $\langle<19|>$ pulse_in $\backslash<19 \mid>$ samp_clk vdd vref) vtc

$|106|<20 \mid>$ (vss vin $|<20|>$ pulse_in $|<20|>$ samp_clk vdd vref) vtc

$\mid 106 \backslash<21 \backslash>$ (vss vin $\mid<21 \backslash>$ pulse_in $\backslash<21 \backslash>$ samp_clk vdd vref) vtc

$|106|<22 \mid>$ (vss vin $|<22|>$ pulse_in $\backslash<22\rangle>$ samp_clk vdd vref) vtc

$\mid 106 \backslash<23 \backslash>$ (vss vin $\backslash<23 \backslash>$ pulse_in $\backslash<23 \backslash>$ samp_clk vdd vref) vtc

$\mid 106 \backslash<24 \backslash>$ (vss vin $\backslash<24 \backslash>$ pulse_in $\backslash<24 \backslash>$ samp_clk vdd vref) vtc

$\mid 106 \backslash<25 \backslash>$ (vss vin $\langle<25 \backslash>$ pulse in $|<25 \backslash>$ samp_clk vdd vref) vtc

$|106|\langle 26|>$ (vss vin $|<26|>$ pulse in $|<26|>$ samp_clk vdd vref) vtc

$1106 \mid<27>$ (vss vin $|<27\rangle$ pulse in $\mid<27>$ samp_clk vdd vref) vtc

$106 \backslash<28)>$ (vss vin $\langle<28|>$ pulse in $\langle<28\rangle$ samp_clk vdd vref) vtc

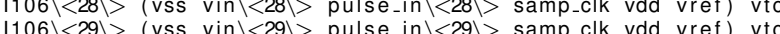

$100 \mid<29>>$ (vss vin $\langle<29|>$ pulse $e_{-}\left|n_{1}\right|<29 \mid>$ samp_clk vdd vref) vtc

C0 (distance vss vss) crtmom $n v=60 \mathrm{nh}=60 \mathrm{w}=140.0 \mathrm{n} \quad \mathrm{s}=140.0 \mathrm{n} \quad \mathrm{stm}=1 \mathrm{spm}=3$ $\mathrm{mf}=10$ mismatchflag=1 busflag=1

V137 $($ win $\backslash<8 \backslash>0$ ) vsource type=pulse edgetype=halfsine val0=0 val1 $=1.2$ period $=30 /$ fsamp delay $=7.5 /$ fsamp $+150 \mathrm{n}$ rise $=200 \mathrm{p}$ fall $=200 \mathrm{p} \backslash$ width $=1 /$ fsamp -200 n

V142 $($ win $\backslash<13 \backslash>0$ ) vsource type=pulse edgetype=halfsine val0=0 val1=1.2 period $=30 /$ fsamp delay $=12.5 /$ fsamp $+150 \mathrm{n}$ rise $=200 \mathrm{p}$ fall $=200 \mathrm{p}$ । width $=1 /$ fsamp -200 n

V141 $($ win $\backslash<12 \backslash>0$ ) vsource type=pulse edgetype=halfsine val0=0 val1=1.2 period $=30 /$ fsamp delay $=11.5 /$ fsamp $+150 \mathrm{n}$ rise $=200 \mathrm{p}$ fall $=200 \mathrm{p}$ । width $=1 /$ fsamp -200 n

V143 ( win $\backslash<14 \backslash>0$ ) vsource type=pulse edgetype=halfsine val0=0 val1=1.2 period $=30 /$ fsamp delay $=13.5 /$ fsamp +150 n rise $=200 p$ fall $=200 p$ width $=1 /$ fsamp -200 n

V144 (win $\backslash<15 \backslash>0$ ) vsource type=pulse edgetype=halfsine val0=0 val1=1.2 period $=30 /$ fsamp delay $=14.5 /$ fsamp $+150 \mathrm{n}$ rise $=200 \mathrm{p}$ fall $=200 \mathrm{p}$ । width $=1$ /fsamp-200n

V136 $($ win $\backslash<7 \backslash>0)$ vsource type=pulse edgetype=halfsine val0=0 val1 $=1.2 \backslash$ period $=30 /$ fsamp delay $=6.5 /$ fsamp +150 n rise $=200 p$ f all $=200 p \backslash$ width $=1 /$ fsamp -200 n

V138 ( $\operatorname{win} \backslash\langle 9 \backslash>0$ ) vsource type=pulse edgetype=halfsine val0=0 val1 $=1.2$ । period $=30 /$ fsamp delay $=8.5 /$ fsamp +150 n rise $=200 p$ fall $=200 p$ । width $=1 /$ fsamp -200 n

V145 $(\operatorname{win} \backslash<30 \backslash>0)$ vsource type=pulse edgetype=halfsine val0=0 val1 $=1.2$ । period $=30 /$ fsamp delay $=29.5 /$ fsamp +150 n rise $=200 p$ fall $=200 p$ । width $=1 /$ fsamp -200 n

V146 ( win $\backslash<29 \backslash>0$ ) vsource type=pulse edgetype=halfsine val0=0 val1=1.2 period $=30 /$ fsamp delay $=28.5 /$ fsamp +150 n rise $=200 p$ fall $=200 p$ width $=1 /$ fsamp -200 n

V147 (win $\backslash<28 \backslash>0$ ) vsource type=pulse edgetype=halfsine val0=0 val1=1.2 period $=30 /$ fsamp delay $=27.5 /$ fsamp +150 n rise $=200$ f fall $=200 p$ । width $=1 /$ fsamp -200 n

V148 ( win $\backslash<27 \backslash>0$ ) vsource type=pulse edgetype=halfsine val0=0 val1=1.2 period $=30 /$ fsamp delay $=26.5 /$ fsamp $+150 \mathrm{n}$ rise $=200 \mathrm{p}$ fall $=200 \mathrm{p}$ । width $=1 /$ fsamp -200 n

V149 ( win $\backslash<26 \backslash>0)$ vsource type=pulse edgetype=halfsine val0=0 val1=1.2 \} period $=30 /$ fsamp delay $=25.5 /$ fsamp $+150 \mathrm{n}$ rise $=200 \mathrm{p}$ fall $=200 \mathrm{p}$ width $=1 /$ fsamp -200 n

V150 $($ win $\backslash<25 \backslash>0)$ vsource type=pulse edgetype=halfsine val0=0 val1 $=1.2 \backslash$ period $=30 / \mathrm{fsamp}$ delay $=24.5 / \mathrm{fsamp}+150 \mathrm{n}$ rise $=200 \mathrm{p}$ fall $=200 \mathrm{p}$ । width $=1 /$ fsamp -200 n

V151 ( win $\backslash<24 \backslash>0$ ) vsource type=pulse edgetype=halfsine val0=0 val1=1.2 \} period $=30 /$ fsamp delay $=23.5 /$ fsamp +150 n rise $=200 p$ fall $=200 p$ । width $=1 /$ fsamp -200 n

V131 $($ win $\backslash<2 \backslash>0)$ vsource type=pulse edgetype=halfsine val0=0 val1=1.2 $\backslash$ period $=30 /$ fsamp delay $=1.5 /$ fsamp +150 n rise $=200 p$ fall $=200 p \backslash$ width $=1 /$ fsamp -200 n

V135 ( win $\backslash<6 \backslash>0$ ) vsource type=pulse edgetype=halfsine val0=0 val1 $=1.2$ period $=30 /$ fsamp delay $=5.5 /$ fsamp +150 n rise $=200$ f fall $=200 p$, width $=1 /$ fsamp -200 n

V139 $($ win $\backslash<10 \backslash>0$ ) vsource type=pulse edgetype=halfsine val0=0 val1=1.2 period $=30 /$ fsamp delay $=9.5 /$ fsamp $+150 \mathrm{n}$ rise $=200 \mathrm{p}$ fall $=200 \mathrm{p} \backslash$ width $=1 /$ fsamp -200 n

V133 $($ win $\backslash<4 \backslash>0)$ vsource type=pulse edgetype=halfsine valo=0 val1 $=1.2$ । period $=30 /$ fsamp delay $=3.5 /$ fsamp $+150 \mathrm{n}$ rise $=200 \mathrm{p}$ fall $=200 \mathrm{p} \backslash$ width $=1 /$ fsamp -200 n 
V132 ( $\operatorname{win} \backslash<3 \backslash>0)$ vsource type=pulse edgetype=halfsine val0=0 val1 $=1.2$ period $=30 /$ fsamp delay $=2.5 /$ fsamp $+150 \mathrm{n}$ rise $=200 \mathrm{p}$ fall $=200 \mathrm{p} \backslash$ width $=1 /$ fsamp -200 n

V109 $($ win $\backslash<1 \backslash>0)$ vsource type=pulse edgetype=halfsine val0=0 val1 $=1.2$ period $=30 /$ fsamp delay $=0.5 /$ fsamp +150 n rise $=200 \mathrm{p}$ fall $=200 p$ width $=1 /$ fsamp -200 n

v98 (samp_clk 0) vsource type=pulse edgetype=halfsine val0=0 val1=1.2 period $=1 /$ fsamp delay $=0.5 /$ fsamp rise $=100 p$ fall $=100 p$ width $=1 \mathrm{u}$

V140 ( win $\backslash<11 \backslash\rangle 0)$ vsource type=pulse edgetype=half $\operatorname{sine}$ val0=0 val1 $=1.2$ period $=30 /$ fsamp delay $=10.5 /$ fsamp +150 n rise $=200 p$ fall $=200 p \backslash$ width $=1$ /fsamp -200 n

V134 ( win $\backslash<5 \backslash>0$ ) vsource type=pulse edgetype=halfsine val0=0 val1=1.2 \} period $=30 /$ fsamp delay $=4.5 /$ fsamp $+150 \mathrm{n}$ rise $=200 \mathrm{p}$ fall $=200 \mathrm{p} \backslash$ width $=1 /$ fsamp -200 n

V152 $($ win $\backslash<23 \backslash>0)$ vsource type=pulse edgetype=halfsine val0=0 val1=1.2 \} period $=30 /$ fsamp delay $=22.5 /$ fsamp $+150 \mathrm{n}$ rise $=200 \mathrm{p}$ fall $=200 \mathrm{p}$ । width $=1 /$ fsamp -200 n

V153 $($ win $\backslash<22 \backslash>0)$ vsource type=pulse edgetype=halfsine val0=0 val1=1.2 \} period $=30 /$ fsamp delay $=21.5 /$ fsamp +150 n rise $=200 p$ fall $=200 p$

V154 ( win $\backslash<21 \backslash>0$ ) vsource type=pulse edgetype=halfsine val0=0 val1=1.2 period $=30 /$ fsamp delay $=20.5 /$ fsamp $+150 \mathrm{n}$ rise $=200 \mathrm{p}$ fall $=200 \mathrm{p} \backslash$ width $=1 /$ fsamp -200 n

V155 (win $\backslash<20\rangle>0$ ) vsource type=pulse edgetype=half $\operatorname{sine}$ val0=0 val1 $=1.2$ period $=30 /$ fsamp delay $=19.5 /$ fsamp $+150 \mathrm{n}$ rise $=200 \mathrm{p}$ fall $=200 \mathrm{p}$ । width 1 /fsamp-200n

V156 ( win $\backslash<19 \backslash>0$ ) vsource type=pulse edgetype=halfsine val0=0 val1 $=1.2$ । period $=30 /$ fsamp delay $=18.5 /$ fsamp $+150 \mathrm{n}$ rise $=200 \mathrm{p}$ fall $=200 \mathrm{p}$ width $=1 /$ fsamp -200 n

V157 ( win $\backslash<18 \backslash>0$ ) vsource type=pulse edgetype=halfsine val0=0 val1 $=1.2$ period $=30 /$ fsamp delay $=17.5 /$ fsamp $+150 \mathrm{n}$ rise $=200 \mathrm{p}$ fall $=200 \mathrm{p}$ । width $=1 /$ fsamp -200 n

V158 $($ win $\backslash<17 \backslash>0)$ vsource type=pulse edgetype=halfsine val0=0 val1 $=1.2$ । period $=30 /$ fsamp delay $=16.5 /$ fsamp +150 n rise $=200 p$ fall $=200 p$ width $=1 /$ fsamp -200 n

V159 ( win $\backslash<16 \backslash>0$ ) vsource type=pulse edgetype=halfsine val0=0 val1=1.2 period $=30 /$ fsamp delay $=15.5 /$ fsamp +150 n rise $=200 p$ fall $=200 p$ । width $=1 /$ fsamp -200 n

V99 ( window 0 ) vsource type $=$ pulse edgetype $=$ halfsine val0 $=1.2$ val $1=0$ period $=1 /$ fsamp delay $=0.5 /$ fsamp rise $=100 p$ fall $=100 p$ । width $=0.25 /$ fsamp

V100 (rst 0) vsource type $=$ pulse edgetype $=$ half $\operatorname{sine}$ val $0=1.2$ val $1=0$ period $=30 /$ fsamp delay $=0.5 /$ fsamp $+1 u$ rise $=100 p$ fall $=100 p$ width $=30 /$ fsamp $-2 u$ ।

$\mid 143 \backslash<1 \backslash>($ en $\backslash<1 \backslash>$ win $\backslash<1 \backslash>$ enb $\backslash<1 \backslash>$ vss vdd) trng_nand

$|143|<2 \mid>($ en $|<2|>$ win $\backslash<2 \mid>$ enb $|<2|>$ vss vdd) trng_nand

$\mid 143 \backslash<3 \backslash>($ en $\backslash<3 \mid>$ win $\backslash<3 \mid>$ enb $\backslash<3 \backslash>$ vss vdd) trng_nand

$\mid 143 \backslash<4 \backslash>($ en $\backslash<4 \mid>$ win $\backslash<4 \backslash>$ enb $\backslash<4 \backslash>$ vss vdd) trng_nand

$|143|<5 \mid>($ en $\mid<5 \backslash>$ win $\mid<5 \backslash>$ enb $\mid<5 \backslash>$ vss vdd) trng_nand

$|143|<6 \mid>($ en $|<6|>$ win $\backslash<6 \mid>$ enb $\backslash<6 \mid>$ vss vdd) trng_nand

$\mid 143 \backslash<7 \backslash>($ en $\mid<7 \backslash>$ win $\backslash<7 \backslash>$ enb $\mid<7 \backslash>$ vss vdd) trng_nand

$|143 \backslash<8|>($ en $|<8|>$ win $\backslash<8 \mid>$ enb $|<8|>$ vss vdd) trng_nand

$|143 \backslash<9|>($ en $|<9|>$ win $\backslash<9 \mid>$ enb $|<9|>$ vss vdd) trng_nand

$\mid 143 \backslash<10 \backslash>($ en $\backslash<10 \backslash>$ win $\backslash<10 \backslash>$ enb $\backslash<10 \backslash>$ vss vdd) trng_nand

$\mid 143 \backslash<11 \backslash>($ en $\backslash<11 \backslash>$ win $\backslash<11 \backslash>$ enb $|<11|>$ vss vdd) trng_nand
$|143 \backslash<12|>$

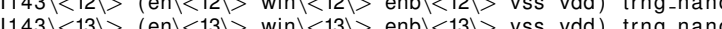

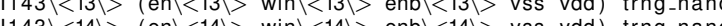

1143 $\mid<14>>($ en $\mid<14>>$ win $|<14|>$ en $|<14|>$ vss vdd) trng_nand

$1143 \mid<15>($ in $|<15|>$ win $|<15|>$ enb $|<15|>$ vss vdd) trng_nand

$|143 \backslash<16|>($ en $|<16|>$ win $|<16|>$ enb $|<16|>$ vss vdd) trng_nand

$1143 \backslash<17 \backslash>($ en $|<17\rangle>$ win $\backslash<17 \backslash>$ enb $\backslash<17 \backslash>$ vss vdd) trng_nand

$|143 \backslash<18|>($ en $|<18|>$ win $|<18|>$ enb $|<18|>$ vss vdd) trng_nand

$|143 \backslash<19|>($ en $|<19|>$ win $|<19|>$ enb $|<19|>$ vss vdd) trng_nand

$|143 \backslash<20|>($ en $|<20|>$ win $\backslash<20 \mid>$ enb $|<20|>$ vss vdd) trng_nand

$|143 \backslash<21|>($ en $\mid<21 \backslash>$ win $\backslash<21 \backslash>$ enb $\mid<21 \backslash>$ vss vdd) trng_nand

$|143|<22 \mid>($ en $|<22|>$ win $\backslash<22 \mid>$ enb $|<22|>$ vss vdd) trng_nand

$|143 \backslash<23|>($ en $\backslash<23 \backslash>$ win $\backslash<23 \backslash>$ enb $\backslash<23 \backslash>$ vss vdd $)$ trng_nand

$|143 \backslash<24|>($ en $\backslash<24 \backslash>$ win $\backslash<24 \backslash>$ enb $\backslash<24 \backslash>$ vss vdd) trng_nand

$\mid 143 \backslash<25 \backslash>($ en $\backslash<25 \backslash>$ win $\backslash<25 \backslash>$ enb $\backslash<25 \backslash>$ vss vdd) trng nand

$|143|<26 \mid>($ en $|<26|>$ win $\backslash<26 \mid>$ enb $|<26|>$ vss vdd) trng_nand

$|143|<27\rangle>($ en $|<27\rangle>$ win $\backslash\langle 27\rangle>$ enb $|<27\rangle>$ vss vdd) trng nand

$|143 \backslash<28|>($ en $|<28|>$ win $|<28|>$ enb $|<28|>$ vss vdd) trng nand

$|143|<29 \mid>($ en $|<29|>$ win $|<29|>$ enb $|<29|>$ vss vdd) trng-nand

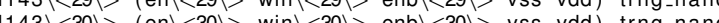

143 $\langle<30|>($ en $\langle<30\rangle>$ win $|<30|>$ enb $|<30|>$

V6 (vref 0) vsource $\mathrm{dc}=400 \mathrm{~m}$ type $=\mathrm{dc}$

V96 ( vin $\backslash<13 \backslash>0)$ vsource dc=Vl13 type $=\mathrm{dc}$

V95 $(\operatorname{vin}|<15|>0)$ vsource $\mathrm{dc}=\mathrm{V} 115$ type $=\mathrm{d}$

V94 $(\operatorname{vin} \backslash<14 \backslash>0)$ vsource $\mathrm{dc}=\mathrm{VI14}$ type $=\mathrm{d}$

V93 $(\operatorname{vin} \backslash<10 \backslash>0)$ vsource $\mathrm{dc}=\mathrm{Vl} 10$ type $=\mathrm{dc}$

V92 $(\operatorname{vin} \backslash<12 \backslash>0)$ vsource $\mathrm{dc}=\mathrm{Vl1}$ type $=\mathrm{dc}$

V91 $(\operatorname{vin} \backslash<11 \backslash>0)$ vsource $d c=V I 11$ type $=d c$

V90 $(\operatorname{vin} \backslash\langle 9\rangle>0)$ vsource $\mathrm{dc}=\mathrm{VI} 9$ type $=\mathrm{dc}$

V82 $(\operatorname{vin}\langle<1\rangle>0)$ vsource $d c=V I 1$ type $=d c$

V81 $(\operatorname{vin} \mid\langle 6 \backslash>0)$ vsource $\mathrm{dc}=\mathrm{VI} 6$ type $=\mathrm{dc}$

V80 $(\operatorname{vin} \backslash<8 \backslash>0)$ vsource $\mathrm{dc}=\mathrm{VI} 8$ type $=\mathrm{dc}$

V79 $(\operatorname{vin} \backslash<7 \backslash>0)$ vsource $\mathrm{dc}=\mathrm{VI} 17$ type $=\mathrm{dc}$

V78 $(\operatorname{vin} \backslash<5 \backslash>0)$ vsource $d c=V I 5$ type $=\mathrm{dc}$

V77 (vin $\langle<3 \backslash>0)$ vsource $d c=V I 3$ type $=d c$

V76 $($ vin $\backslash<4 \mid>0)$ vsource $d c=V I 4$ type $=d c$

V75 (vin $\langle<2>0$ ) vsource $d c=V 14$ type $=d c$

V75 $(v i n\langle 2<0)$ vsource $d c=V 12$ type $=\mathrm{dc}$

V66 $(v \operatorname{vet}|<20|>0)$ vsource $d c=V C 20$ type $=d c$

V65 (vctr $\mid\langle<22 \backslash>0)$ vsource $d c=V C 22$ type $=d$

V64 $(\operatorname{vctr} I \mid<23 \backslash>0)$ vsource $d c=V C 23$ type $=d c$

V63 $(v \operatorname{vetr} I \mid<21 \backslash>0)$ vsource $d c=V C 21$ type $=d c$

V62 (vctr $\mid\langle<17\rangle>0)$ vsource dc=VC17 type $=d c$

V61 $(v \operatorname{vet} \mid \backslash\langle 19 \backslash>0)$ vsource dc=VC19 type $=\mathrm{dc}$

V60 $(v \operatorname{vetr} \mid\langle<18 \backslash>0)$ vsource $d c=V C 18$ type $=d c$

V59 $(\operatorname{vctr} I \backslash<16 \backslash>0)$ vsource dc=VC16 type $=d c$ 
$\begin{array}{lll}\text { V47 }(v \operatorname{tr} I \backslash<24 \backslash>0) & \text { vsource } d c=V C 24 \text { type }=d c \\ \text { V46 }(\text { vctr } \mid \backslash<26 \backslash>0) & \text { vsource } d c=V C 26 \text { type }=d c\end{array}$

V45 $(v \operatorname{vetr} \mid\langle<25 \backslash>0)$ vsource $d c=V C 25$ type $=d c$

V44 $(v \operatorname{vtr} \mid \backslash<28 \backslash>0)$ vsource $d c=V C 28$ type $=d c$

V43 (vctrl $<<30 \backslash>0)$ vsource $d c=V C 30$ type $=d c$

V42 (vctrl $<29>0)$ vsource dc $=$ V 30 type $=\mathrm{dc}$

V42 (vctr $|<29|>0)$ vsource $d c=V C 29$ type $=d c$

V41 (vctr $\backslash\langle<27 \backslash>0)$ vsource $d c=V C 27$ type $=d$

V89 (vin $|<26|>0)$ vsource $\mathrm{dc}=\mathrm{VI} 26$ type $=\mathrm{dc}$

V88 $(\operatorname{vin} \backslash<25 \backslash>0)$ vsource $\mathrm{dc}=\mathrm{VI} 25$ type $=\mathrm{dc}$

V87 $(\operatorname{vin} \backslash<28 \backslash>0)$ vsource $\mathrm{dc}=\mathrm{VI} 28$ type $=\mathrm{dc}$

V86 $(\operatorname{vin} \backslash<30 \backslash>0)$ vsource $\mathrm{dc}=\mathrm{VI} 30$ type $=\mathrm{dc}$

V85 $(\operatorname{vin} \backslash<29 \backslash>0)$ vsource $d c=V I 29$ type $=d c$

V84 $(\operatorname{vin} \mid<27 \backslash>0)$ vsource $d c=V I 27$ type $=d c$

V74 $(\operatorname{vin} \backslash<22\rangle>0)$ vsource $d c=V I 22$ type $=d c$

V73 $(\operatorname{vin} \backslash<23 \backslash>0)$ vsource dc=VI23 type $=d c$

V72 $(\operatorname{vin} \backslash<21 \backslash>0)$ vsource $d c=V I 21$ type $=d c$

V71 $(\operatorname{vin} \backslash<17 \backslash>0)$ vsource dc=VI17 type $=d c$

V70 $($ vin $\backslash<19 \backslash>0)$ vsource $d c=V 119$ type $=d c$

V69 $(\operatorname{vin} \backslash<18 \backslash>0)$ vsource $d c=V I 18$ type $=d c$

V68 $(\operatorname{vin} \backslash\langle 16\rangle>0)$ vsource $d c=V \mid 16$ type $=d c$

V67 $(\operatorname{vin} \backslash<20 \backslash>0)$ vsource $\mathrm{dc}=\mathrm{VI} 20$ type $=\mathrm{dc}$

V37 (vctr $\mid \backslash<13 \backslash>0)$ vsource dc=VC13 type=dc

V37 $(v c \operatorname{tr} \mid\langle<13 \backslash>0)$ vsource $d c=V C 13$ type $=d c$

V36 ( vctr $\mid \backslash<15 \backslash>0)$ vsource $d c=V C 15$ type $=d c$

V34 (vctr $\mid\langle<14 \backslash>0)$ vsource dc=VC14 type $=d c$

V33 $(\operatorname{vctr} \mid\langle<10 \backslash>0)$ vsource $d c=V C 10$ type $=d c$

V32 (vctrl $\mid<12 \backslash>0)$ vsource $d c=V C 12$ type $=d c$

V31 $(v \operatorname{vat} \mid \backslash<11 \backslash>0)$ vsource $d c=V C 11$ type $=d$

V30 (vctrl $\langle<9 \backslash>0)$ vsource $d c=V C 9$ type $=d c$

V83 ( vin $\backslash<24 \backslash>0)$ vsource $d c=V I 24$ type $=d c$

V4 (vdd vss) vsource $\mathrm{dc}=1.2$ type $=\mathrm{dc}$

V3 (vss 0 ) vsource $\mathrm{dc}=0$ type $=\mathrm{dc}$

V8 $(\operatorname{vctr} I \mid<1 \backslash>0)$ vsource dc=VC1 type $=$ dc

V21 (vctr ||$<6 \backslash>0$ ) vsource dc=VC6 type $=d c$

V20 $(v \operatorname{vet}||\langle 8\rangle>0)$ vsource $d c=V C 8$ type $=d c$

V19 $(v \operatorname{vet}||\langle 7\rangle>0)$ vsource dc=VC7 type $=d c$

V18 (vctrl $\mid<5 \backslash>0)$ vsource dc=VC5 type $=$ dc

V11 $(v \operatorname{vat} \mid \backslash\langle 3\rangle>0)$ vsource dc=VC3 type $=\mathrm{dc}$

V10 ( vctrl $|<4|>0)$ vsource $d c=V C 4$ type $=$ dc

$V 10$
$v 9(v \operatorname{vat} I|<2\rangle>0)$

V9 $($ v ctrl $|<2|>0)$ vsource dc=VC2 type=dc

$|11|<1 \mid>$ (pulse_in $\backslash<1 \backslash>$ pulse_out $\mid<1 \backslash>$ vctrl $|<1\rangle>$ vdd vss) delay4

$|11|<2\rangle>$ (pulse_in $|<2\rangle>$ pulse_out $|<2|>$ vctrl $|<2\rangle>$ vdd vss) delay 4

$|11|<3 \backslash>$ (pulse_in $\mid<3 \backslash>$ pulse_out $\mid<3 \backslash>$ vctrl $\mid<3 \backslash>$ vdd vss) delay 4

$|11|<4 \backslash>$ (pulse_in $\mid<4 \backslash>$ pulse_out $\mid<4 \backslash>$ vctrl $\mid<4 \backslash>$ vdd vss) delay 4

$|11|<5 \backslash>$ (pulse_in $\mid<5 \backslash>$ pulse_out $\mid<5 \backslash>$ vctrl $\mid<5 \backslash>$ vdd vss) delay 4

$|11|<6 \mid>$ (pulse_in $\mid<6 \backslash>$ pulse_out $|<6|>$ vctrl $|<6|>$ vdd vss) delay4

$|11|<7\rangle>$ (pulse_in $|<7\rangle>$ pulse_out $|<7|>$ vctrl $|<7|>$ vdd vss) delay4

$|11|<8 \mid>$ (pulse_in $|<8|>$ pulse_out $|<8|>$ vctrl $|<8|>$ vdd vss) delay 4

$|11|<9 \mid>$ (pulse_in $|<9|>$ pulse_out $|<9|>$ vctrl $|<9|>$ vdd vss) delay4

$\mid 11 \backslash<10 \backslash>$ (pulse_in $\backslash<10 \backslash>$ pulse_out $\backslash<10 \backslash>$ vctrl $\backslash<10 \backslash>$ vdd vss) delay 4

$|11|<11 \mid>$ (pulse_in $|<11|>$ pulse_out $|<11|>$ vctrl $\mid\langle 11|>$ vdd vss) delay 4

$|11|<12\rangle>$ (pulse_in $|<12|>$ pulse_out $|<12|>$ vctrl $|<12|>$ vdd vss) delay 4

$|11|<13 \backslash>$ (pulse_in $\backslash<13 \backslash>$ pulse_out $\mid<13 \backslash>$ vctr $\mid\langle<13 \backslash>$ vdd vss) delay 4

$|11|<14 \mid>$ (pulse_in $\backslash<14 \backslash>$ pulse_out $|<14|>$ vctrl $|<14|>$ vdd vss) delay 4

$|11|<15\rangle>$ (pulse_in $\backslash<15 \backslash>$ pulse_out $|<15|>$ vctrl $|<15|>$ vdd vss) delay 4

$|11|\langle 16\rangle$ (pulse in $|<16\rangle>$ pulse out $\mid\langle 16\rangle>$ vatr| $|<16|>$ vdd vss) delay 4

$111<<16>>$ (pulse_in $\langle<16>>$ pulse_out $\langle<1<>$ vctr $|<16>>$ vdd vss) delay 4

$111|<17\rangle>$ (pulse_in $|<17\rangle>$ pulse_out $|<17|>v \operatorname{vetr} \mid<17 \backslash>$ vdd vss) delay 4

$\mid 11 \backslash<18 \backslash>$ (pulse_in $\backslash<18 \backslash>$ pulse_out $\backslash<18 \backslash>$ vctr $\mid \backslash<18 \backslash>$ vdd vss) delay 4

$|11|<19 \mid>$ (pulse in $|<19|>$ pulse_out $|<19|>$ vctr $\mid\langle<19|>$ vdd vss) delay 4

$111|<20|>$ (pulse _in $|<20|>$ pulse_out $|<20|>$ vctr $|<20|>$ vad vss) delay 4

$111 \backslash<21 \backslash>$ (pulse_in $\backslash<21 \backslash>$ pulse_out $\mid<21 \backslash>$ vctr $\mid\langle 21 \backslash>$ vdd vss) delay 4

$|11|<22 \mid>$ (pulse_in $|<22|>$ pulse_out $|<22|>$ vctrl $|<22|>$ vdd vss) delay 4

$\mid 11 \backslash<23 \backslash>$ (pulse in $|<23|>$ pulse put $|<23|>$ vctrl $\mid<23 \backslash>$ vdd vss) delay 4

$|11 \backslash<24|>$ (pulse_in $|<24|>$ pulse_out $|<24|>$ vctrl $|<24|>$ vdd vss) delay 4

$|11|<25 \mid>$ (pulse_in $\mid<25 \backslash>$ pulse_out $|<25|>$ vctrl $|<25|>$ vdd vss) delay 4

$|11|<26 \mid>$ (pulse_in $|<26|>$ pulse_out $|<26|>$ vctrl $|<26|>$ vdd vss) delay 4

$|11|<27 \backslash>$ (pulse_in $\backslash<27 \backslash>$ pulse_out $\backslash<27 \backslash>$ vctrl $\mid<27 \backslash>$ vdd vss) delay 4

$|11|<28 \mid>$ (pulse_in $|<28|>$ pulse_out $\mid<28 \backslash>$ vctrl $\mid<28 \backslash>$ vdd vss) delay 4

$|11|<29 \mid>$ (pulse_in $\backslash<29 \mid>$ pulse_out $|<29|>$ vctrl $|<29|>$ vdd vss) delay 4

$|11|\langle 30|>$ (pulse_in $\backslash<30 \backslash>$ pulse_out $\backslash<30 \backslash>$ vctrI $\backslash<30 \backslash>$ vdd vss) delay 4

M3 (distance rst vss vss) nch_hvt_mac $I=1 \mathrm{u} \quad \mathrm{w}=5 \mathrm{u}$ multi $=(1) \mathrm{nf}=1 \mathrm{sd}=260.0 \mathrm{n}$ $\mathrm{ad}=1.15 \mathrm{e}-12$ as $=1.15 \mathrm{e}-12 \mathrm{pd}=10.46 \mathrm{u} \mathrm{ps}=10.46 \mathrm{u} \mathrm{nrd}=0.026 \mathrm{nrs}=0.026$ । $\mathrm{ad}=1.15 \mathrm{e}-12$ as $=1.15 \mathrm{e}-12 \mathrm{pd}=10.46 \mathrm{u} \mathrm{ps}=10.46 \mathrm{u} \mathrm{nrd}=0.026 \mathrm{nrs}=0.026$

1104 (net025 vss) isource dc=25n type $=$ dc

M1 (A net025 vdd vdd) pch_hvt_mac $I=5 \mathrm{u} \quad \mathrm{w}=1 \mathrm{u}$ multi=(1) $\mathrm{nf}=1 \quad \mathrm{sd}=260.0 \mathrm{n}$ ) $\mathrm{ad}=2.3 \mathrm{e}-13$ as $=2.3 \mathrm{e}-13 \mathrm{pd}=2.46 \mathrm{u} \mathrm{ps}=2.46 \mathrm{u}$ nrd $=0.13 \mathrm{nrs}=0.13$ । $\mathrm{sa}=230.0 \mathrm{n} \quad \mathrm{sb}=230.0 \mathrm{n} \quad \mathrm{sca}=0 \quad \mathrm{scb}=0 \quad \mathrm{scc}=0$ mismatchflag $=1$ sigma $=1$

MO (net025 net025 vdd vdd) pch_hvt_mac $I=5 \mathrm{u} \quad \mathrm{W}=1 \mathrm{u}$ multi=(1) $\mathrm{nf}=1$ sd=260.0n $\mathrm{ad}=2.3 \mathrm{e}-13$ as $=2.3 \mathrm{e}-13 \mathrm{pd}=2.46 \mathrm{u}$ ps $=2.46 \mathrm{u}$ nrd $=0.13 \mathrm{nrs}=0.13$ । $\mathrm{sa}=230.0 \mathrm{n} \quad \mathrm{sb}=230.0 \mathrm{n} \quad \mathrm{sca}=0 \quad \mathrm{scb}=0 \quad \mathrm{scc}=0$ mismatchflag $=1$ sigma $=1$

$\mathrm{M} 2\langle\langle 1\rangle$ (distance enb $\langle<1 \backslash>A$ vdd) pch_hvt_mac $I=1 \mathrm{u} \quad \mathrm{w}=1 \mathrm{u}$ multi $=(1) \mathrm{nf}=1\rangle$ $\mathrm{sd}=260.0 \mathrm{n}$ ad $=2.3 \mathrm{e}-13$ as $=2.3 \mathrm{e}-13 \mathrm{pd}=2.46 \mathrm{u}$ ps $=2.46 \mathrm{u} \mathrm{nrd}=0.13$ $\mathrm{nrs}=0.13 \mathrm{sa}=230.0 \mathrm{n} \quad \mathrm{sb}=230.0 \mathrm{n} \quad \mathrm{sca}=0 \quad \mathrm{scb}=0 \quad \mathrm{scc}=0$ mismatchflag $=1$ । sigma $=1$

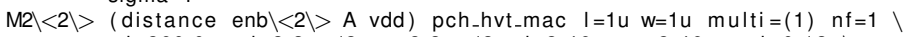
$\mathrm{sd}=260.0 \mathrm{n}$ ad $=2.3 \mathrm{e}-13$ as $=2.3 \mathrm{e}-13 \mathrm{pd}=2.46 \mathrm{u} \mathrm{ps}=2.46 \mathrm{u} \mathrm{nrd}=0.13$ $\mathrm{nrs}=0.13 \mathrm{sa}=230.0 \mathrm{n} \mathrm{sb}=230.0 \mathrm{n} \mathrm{sca}=0 \mathrm{scb}=0 \mathrm{scc}=0$ mismatchflag $=1$ sigma $=1$

$\mathrm{M} 2 \backslash\langle 3 \backslash>$ (distance enb $\backslash<3 \backslash>A$ vdd) pch_hvt_mac $\mathrm{I}=1 \mathrm{u}$ w=1u multi=(1) $n f=1 \backslash$ $\mathrm{sd}=260.0 \mathrm{n}$ ad $=2.3 \mathrm{e}-13$ as $=2.3 \mathrm{e}-13 \mathrm{pd}=2.46 \mathrm{u} \mathrm{ps}=2.46 \mathrm{u} \mathrm{nrd}=0.13$ $\mathrm{nrs}=0.13$ sa $=230.0 \mathrm{n} \quad \mathrm{sb}=230$. 0 n $\mathrm{sca}=0 \quad \mathrm{scb}=0 \quad \mathrm{scc}=0$ mismatchflag $=1$ $\mathrm{nrs}=0.13$
sigma $=1$

$\mathrm{M} 2 \backslash<4 \backslash>($ distance enb $\backslash<4 \backslash>A$ vdd) pch_hvt_mac $\mathrm{I}=1 \mathrm{u} \quad \mathrm{w}=1 \mathrm{u}$ multi $=$ (1) $n f=1$ $\mathrm{sd}=260.0 \mathrm{n}$ ad $=2.3 \mathrm{e}-13$ as $=2.3 \mathrm{e}-13 \mathrm{pd}=2.46 \mathrm{u}$ ps $=2.46 \mathrm{u} \mathrm{nrd}=0.13$ $\mathrm{nrs}=0.13 \mathrm{sa}=230.0 \mathrm{n} \mathrm{sb}=230.0 \mathrm{n}$ sca $=0 \mathrm{scb}=0 \mathrm{scc}=0$ mismatchflag $=1$ । sigma $=1$

$\mathrm{M} 2 \backslash<5 \backslash>$ (distance enb $\backslash<5 \backslash>A$ vdd) pch_hvt_mac $\mathrm{I}=1 \mathrm{u} \quad \mathrm{w}=1 \mathrm{u}$ multi $=$ (1) $n f=1\rangle$ $\mathrm{sd}=260.0 \mathrm{n}$ ad $=2.3 \mathrm{e}-13$ as $=2.3 \mathrm{e}-13 \mathrm{pd}=2.46 \mathrm{u} \mathrm{ps}=2.46 \mathrm{u} \mathrm{nrd}=0.13$ । $\mathrm{nrs}=0.13 \mathrm{sa}=230.0 \mathrm{n} \quad \mathrm{sb}=230.0 \mathrm{n}$ sca $=0 \quad \mathrm{scb}=0 \quad \mathrm{scc}=0$ mismatchflag=1 
sigma $=1$

M2 $\langle<|>$ (distance enb $\backslash\langle 6 \backslash>A$ vdd) pch_hvt_mac $\mathrm{I}=1 \mathrm{u} \quad \mathrm{w}=1 \mathrm{u}$ multi $=(1) \mathrm{nf}=1$ $\mathrm{sd}=260.0 \mathrm{n}$ ad $=2.3 \mathrm{e}-13$ as $=2.3 \mathrm{e}-13 \mathrm{pd}=2.46 \mathrm{u} \mathrm{ps}=2.46 \mathrm{u} \mathrm{nrd}=0.13$ $\mathrm{nrs}=0.13 \mathrm{sa}=230.0 \mathrm{n} \mathrm{sb}=230.0 \mathrm{n}$ sca=0 $\mathrm{scb}=0 \mathrm{scc}=0$ mismatchflag=1 sigma $=1$

$M 2 \backslash\langle 7\rangle\rangle$ (distance enb $\langle\langle 7\rangle>$ A vdd) pch_hvt_mac $\mathrm{I}=1 \mathrm{u} \quad \mathrm{w}=1 \mathrm{u}$ multi $=(1) \mathrm{nf}=1$ $\mathrm{sd}=260.0 \mathrm{n}$ ad $=2.3 \mathrm{e}-13$ as $=2.3 \mathrm{e}-13 \mathrm{pd}=2.46 \mathrm{u}$ ps $=2.46 \mathrm{u} \mathrm{nrd}=0.13$ । $\mathrm{sd}=260.0 \mathrm{n}$ ad $=2.3 \mathrm{e}-13 \mathrm{as}=2.3 \mathrm{e}-13 \mathrm{pd}=2.46 \mathrm{u}$ ps $=2.46 \mathrm{u} \mathrm{nrd}=0.13$
$\mathrm{nrs}=0.13$ sa $=230.0 \mathrm{n}$ sb $=230.0 \mathrm{n}$ sca $=0 \quad \mathrm{scb}=0$ scc $=0$ mismatchflag $=1$ $\mathrm{nrs}=0.13$
sigma $=1$

$\mathrm{M} 2\rangle\langle 8\rangle>$ (distance enb $\backslash<8 \backslash>$ A vdd) pch_hvt_mac $\mathrm{I}=1 \mathrm{u} \quad \mathrm{w}=1 \mathrm{u}$ multi $=(1) \mathrm{nf}=1\rangle$ $\mathrm{sd}=260.0 \mathrm{n}$ ad $=2.3 \mathrm{e}-13$ as $=2.3 \mathrm{e}-13 \mathrm{pd}=2.46 \mathrm{u} \mathrm{ps}=2.46 \mathrm{u} \mathrm{nrd}=0.13$ $\mathrm{nrs}=0.13 \mathrm{sa}=230.0 \mathrm{n} \quad \mathrm{sb}=230.0 \mathrm{n}$ sca=0 $\mathrm{scb}=0 \mathrm{scc}=0$ mismatchflag=1 । sigma $=1$

M2 $|<9|>$ (distance enb $\backslash<9 \backslash>A$ vdd) pch_hvt_mac $I=1 \mathrm{u} \quad \mathrm{w}=1 \mathrm{u}$ multi=(1) $n f=1$ $\mathrm{sd}=260.0 \mathrm{n}$ ad $=2.3 \mathrm{e}-13$ as $=2.3 \mathrm{e}-13 \mathrm{pd}=2.46 \mathrm{u}$ ps $=2.46 \mathrm{u} \mathrm{nrd}=0.13$ । $\mathrm{nrs}=0.13 \mathrm{sa}=230.0 \mathrm{n} \mathrm{sb}=230$. 0 n $\mathrm{sca}=0 \mathrm{scb}=0 \quad \mathrm{scc}=0$ mismatchflag $=1$ । sigma $=1$

$\mathrm{M} 2 \backslash<10 \backslash>$ (distance enb $\backslash<10 \backslash>\mathrm{A}$ vdd) pch_hvt_mac $\mathrm{I}=1 \mathrm{u} \mathrm{w}=1 \mathrm{u}$ multi=(1) $\mathrm{nf}=1$ $\mathrm{sd}=260.0 \mathrm{n} \mathrm{ad}=2.3 \mathrm{e}-13$ as $=2.3 \mathrm{e}-13 \mathrm{pd}=2.46 \mathrm{u} \mathrm{ps}=2.46 \mathrm{u} \mathrm{nrd}=0.13$ । $n r s=0.13 \mathrm{sa}=230.0 \mathrm{n} \quad \mathrm{sb}=230.0 \mathrm{n}$ sca $=0 \mathrm{scb}=0 \mathrm{scc}=0$ mismatchflag $=1$ sigma $=1$

$\mathrm{M} 2\rangle\langle 11 \backslash\rangle$ (distance enb $\backslash\langle 11 \backslash\rangle$ A vdd) pch_hvt_mac $\mathrm{I}=1 \mathrm{u} \mathrm{w}=1 \mathrm{u}$ multi=(1) $\mathrm{nf}=1$ $\mathrm{sd}=260.0 \mathrm{n}$ ad $=2.3 \mathrm{e}-13$ as $=2.3 \mathrm{e}-13 \mathrm{pd}=2.46 \mathrm{u}$ ps $=2.46 \mathrm{u} \mathrm{nrd}=0.13$ । $\mathrm{sd}=260.0 \mathrm{n} \quad \mathrm{ad}=2.3 \mathrm{e}-13 \mathrm{as}=2.3 \mathrm{e}-13 \mathrm{pd}=2.46 \mathrm{u} \quad \mathrm{ps}=2.46 \mathrm{u} \mathrm{nrd}=0.13$
$\mathrm{nrs}=0.13 \mathrm{sa}=230.0 \mathrm{n} \quad \mathrm{sb}=230.0 \mathrm{n} \quad \mathrm{sca}=0 \quad \mathrm{scb}=0 \quad \mathrm{scc}=0$ mismatchflag $=1$ $\mathrm{nrs}=0.13$
sigma $=1$

$\mathrm{M} 2 \backslash\langle 12 \backslash>$ (distance enb $\backslash<12 \backslash>A$ vdd) pch_hvt_mac $\mathrm{I}=1 \mathrm{u} \mathrm{w}=1 \mathrm{u}$ multi=(1) $\mathrm{nf}=1$ $\mathrm{sd}=260.0 \mathrm{n}$ ad $=2.3 \mathrm{e}-13$ as $=2.3 \mathrm{e}-13 \mathrm{pd}=2.46 \mathrm{u}$ ps $=2.46 \mathrm{u} \mathrm{nrd}=0.13$ $\mathrm{nrs}=0.13 \mathrm{sa}=230.0 \mathrm{n}$ sb=230.0n $\mathrm{sca}=0 \mathrm{scb}=0 \mathrm{scc}=0$ mismatchflag=1 sigma $=1$

$\mathrm{M} 2 \backslash<13 \backslash>$ (distance enb $\backslash<13 \backslash>A$ vdd) pch_hvt_mac $\mathrm{I}=1 \mathrm{u} \mathrm{w}=1 \mathrm{u}$ multi=(1) $\mathrm{nf}=1$ $\mathrm{sd}=260.0 \mathrm{n}$ ad $=2.3 \mathrm{e}-13$ as $=2.3 \mathrm{e}-13 \mathrm{pd}=2.46 \mathrm{u}$ ps $=2.46 \mathrm{u} \mathrm{nrd}=0.13$ । $\mathrm{nrs}=0.13 \mathrm{sa}=230.0 \mathrm{n} \mathrm{sb}=230.0 \mathrm{n} \quad \mathrm{sca}=0 \mathrm{scb}=0$ scc=0 mismatchflag=1 sigma $=1$

$\mathrm{M} 2 \backslash<14 \backslash>$ (distance enb $\backslash<14 \backslash>\mathrm{A}$ vdd) pch_hvt_mac $\mathrm{I}=1 \mathrm{u}$ w=1u multi=(1) $n f=1$ $\mathrm{sd}=260.0 \mathrm{n}$ ad $=2.3 \mathrm{e}-13 \mathrm{as}=2.3 \mathrm{e}-13 \mathrm{pd}=2.46 \mathrm{u} \mathrm{ps}=2.46 \mathrm{u} \mathrm{nrd}=0.13$ $n r s=0.13 \mathrm{sa}=230.0 \mathrm{n} \mathrm{sb}=230.0 \mathrm{n}$ sca $=0 \mathrm{scb}=0 \mathrm{scc}=0$ mismatchflag=1 sigma $=1$

M2 $\langle<15 \backslash>$ (distance enb $\backslash<15 \backslash>A$ vdd) pch_hvt_mac I=1u w=1u multi=(1) nf=1 $\mathrm{sd}=260.0 \mathrm{n}$ ad $=2.3 \mathrm{e}-13$ as $=2.3 \mathrm{e}-13 \mathrm{pd}=2.46 \mathrm{u}$ ps $=2.46 \mathrm{u} \mathrm{nrd}=0.13$ $\mathrm{nrs}=0.13$ sa=230.0n $\mathrm{sb}=230.0 \mathrm{n} \quad \mathrm{sca}=0 \quad \mathrm{scb}=0 \quad \mathrm{scc}=0$ mismatchflag=1 $\mathrm{nrs}=0.13$
sigma $=1$

M2 $\langle<16 \backslash>$ (distance enb $\backslash\langle 16 \backslash>A$ vdd) pch_hvt_mac $\mathrm{I}=1 \mathrm{u}$ w=1u multi=(1) $n f=1\rangle$ $\mathrm{sd}=260.0 \mathrm{n} \mathrm{ad}=2.3 \mathrm{e}-13$ as $=2.3 \mathrm{e}-13 \mathrm{pd}=2.46 \mathrm{u}$ ps $=2.46 \mathrm{u} \mathrm{nrd}=0.13$ $\mathrm{nrs}=0.13 \mathrm{sa}=230.0 \mathrm{n}$ sb=230.0n $\mathrm{sca}=0 \mathrm{scb}=0 \mathrm{scc}=0$ mismatchflag=1 sigma $=1$

$\mathrm{M} 2 \backslash<17 \backslash>$ (distance enb $\backslash<17 \backslash>A$ vdd) pch_hvt_mac $\mathrm{I}=1 \mathrm{u} \quad \mathrm{w}=1 \mathrm{u}$ multi=(1) $n f=1\rangle$ $\mathrm{sd}=260.0 \mathrm{n}$ ad $=2.3 \mathrm{e}-13$ as $=2.3 \mathrm{e}-13 \mathrm{pd}=2.46 \mathrm{u}$ ps $=2.46 \mathrm{u} \mathrm{nrd}=0.13$ । $\mathrm{nrs}=0.13 \mathrm{sa}=230.0 \mathrm{n} \quad \mathrm{sb}=230.0 \mathrm{n}$ sca $=0 \quad \mathrm{scb}=0 \quad \mathrm{scc}=0$ mismatchflag $=1$ । sigma $=1$

$\mathrm{M} 2\langle<18 \backslash>$ (distance enb $\backslash<18 \backslash>$ A vdd) pch_hvt_mac $\mathrm{I}=1 \mathrm{u} \mathrm{w}=1 \mathrm{u}$ multi=(1) $\mathrm{nf}=1$ $\mathrm{sd}=260.0 \mathrm{n}$ ad $=2.3 \mathrm{e}-13 \mathrm{as}=2.3 \mathrm{e}-13 \mathrm{pd}=2.46 \mathrm{u}$ ps $=2.46 \mathrm{u} \mathrm{nrd}=0.13$ $\mathrm{nrs}=0.13 \mathrm{sa}=230.0 \mathrm{n} \mathrm{sb}=230.0 \mathrm{n}$ sca $=0 \mathrm{scb}=0 \mathrm{scc}=0$ mismatchflag=1 sigma $=1$

$\mathrm{M} 2\langle<19 \backslash>$ (distance enb $\backslash<19 \backslash>$ A vdd) pch_hvt_mac I=1u w=1u multi=(1) $n f=1$ $\mathrm{sd}=260.0 \mathrm{n}$ ad $=2.3 \mathrm{e}-13$ as $=2.3 \mathrm{e}-13 \mathrm{pd}=2.46 \mathrm{u}$ ps $=2.46 \mathrm{u} \mathrm{nrd}=0.13$ $\mathrm{nrs}=0.13$ sa $=230.0 \mathrm{n} \quad \mathrm{sb}=230.0 \mathrm{n} \quad \mathrm{sca}=0 \quad \mathrm{scb}=0 \quad \mathrm{scc}=0$ mismatchflag=1। $\mathrm{nrs}=0.13$
$\mathrm{sigma}=1$

$\mathrm{M} 2 \backslash\langle 20 \backslash\rangle$ (distance enb $\backslash<20 \backslash>\mathrm{A}$ vdd) pch_hvt_mac I=1u w=1u multi=(1) $n f=1$ $\mathrm{sd}=260.0 \mathrm{n}$ ad $=2.3 \mathrm{e}-13$ as $=2.3 \mathrm{e}-13 \mathrm{pd}=2.46 \mathrm{u}$ ps $=2.46 \mathrm{u}$ nrd $=0.13$ । $\mathrm{nrs}=0.13 \mathrm{sa}=230.0 \mathrm{n} \quad \mathrm{sb}=230.0 \mathrm{n} \quad \mathrm{sca}=0 \quad \mathrm{scb}=0 \quad \mathrm{scc}=0$ mismatchflag $=1$ sigma $=1$

$\mathrm{M} 2 \backslash\langle 21 \backslash>$ (distance enb $\backslash<21 \backslash>A$ vdd) pch_hvt_mac $\mathrm{I}=1 \mathrm{u} \mathrm{w}=1 \mathrm{u}$ multi=(1) $n f=1\rangle$ $\mathrm{sd}=260.0 \mathrm{n}$ ad $=2.3 \mathrm{e}-13$ as $=2.3 \mathrm{e}-13 \mathrm{pd}=2.46 \mathrm{u} \mathrm{ps}=2.46 \mathrm{u} \mathrm{nrd}=0.13$ $\mathrm{nrs}=0.13 \mathrm{sa}=230.0 \mathrm{n} \quad \mathrm{sb}=230.0 \mathrm{n} \quad \mathrm{sca}=0 \quad \mathrm{scb}=0 \quad \mathrm{scc}=0$ mismatchflag $=1$ । sigma $=1$

$\mathrm{M} 2 \backslash<22\rangle>$ (distance enb $\backslash<22\rangle>$ A vdd) pch_hvt_mac $\mathrm{I}=1 \mathrm{u}$ w=1u multi=(1) $\mathrm{nf}=1$ $\mathrm{sd}=260.0 \mathrm{n}$ ad $=2.3 \mathrm{e}-13 \mathrm{as}=2.3 \mathrm{e}-13 \mathrm{pd}=2.46 \mathrm{u} \mathrm{ps}=2.46 \mathrm{u} \mathrm{nrd}=0.13$ $\mathrm{nrs}=0.13 \mathrm{sa}=230.0 \mathrm{n} \mathrm{sb}=230.0 \mathrm{n}$ sca $=0 \mathrm{scb}=0 \mathrm{scc}=0$ mismatchflag $=1$ । sigma $=1$

$\mathrm{M} 2 \backslash<23 \backslash>$ (distance enb $\backslash<23 \backslash>$ A vdd) pch_hvt_mac I=1u w=1u multi=(1) $n f=1$ $\mathrm{sd}=260.0 \mathrm{n}$ ad $=2.3 \mathrm{e}-13$ as $=2.3 \mathrm{e}-13 \mathrm{pd}=2.46 \mathrm{u}$ ps $=2.46 \mathrm{u} \mathrm{nrd}=0.13$ । $\mathrm{nrs}=0.13 \mathrm{sa}=230.0 \mathrm{n} \mathrm{sb}=230.0 \mathrm{n} \quad \mathrm{sca}=0 \mathrm{scb}=0 \quad \mathrm{scc}=0$ mismatchflag=1 $\mathrm{nrs}=0.13$
sigma $=1$

M2 $\backslash<24 \backslash>$ (distance enb $\backslash<24 \backslash>$ A vdd) pch_hvt_mac I=1u w=1u multi=(1) $n f=1$ $\mathrm{sd}=260.0 \mathrm{n}$ ad $=2.3 \mathrm{e}-13$ as $=2.3 \mathrm{e}-13 \mathrm{pd}=2.46 \mathrm{u}$ ps $=2.46 \mathrm{u} \mathrm{nrd}=0.13$ । $\mathrm{nrs}=0.13$ sa $=230.0 \mathrm{n} \quad \mathrm{sb}=230.0 \mathrm{n} \quad \mathrm{sca}=0 \quad \mathrm{scb}=0 \quad \mathrm{scc}=0$ mismatchflag $=1$ $\mathrm{nrs}=0.13$
$\mathrm{sigma}=1$

$\mathrm{M} 2 \backslash<25 \backslash>($ distance enb $\backslash<25 \backslash>\mathrm{A}$ vdd) pch_hvt_mac $\mathrm{I}=1 \mathrm{u} \mathrm{w}=1 \mathrm{u}$ multi=(1) $\mathrm{nf}=1 \backslash$ $\mathrm{sd}=260.0 \mathrm{n}$ ad=2.3e -13 as $=2.3 \mathrm{e}-13 \mathrm{pd}=2.46 \mathrm{u} \mathrm{ps}=2.46 \mathrm{u} \mathrm{nrd}=0.13$ $\mathrm{nrs}=0.13 \mathrm{sa}=230.0 \mathrm{n} \mathrm{sb}=230$. 0 n sca=0 $\mathrm{scb}=0$ scc=0 mismatchflag=1 sigma $=1$

$\mathrm{M} 2\langle<26 \backslash>$ (distance enb $\backslash<26 \backslash>$ A vdd) pch_hvt_mac $\mathrm{I}=1 \mathrm{u}$ w=1u multi=(1) $n f=1\rangle$ $\mathrm{sd}=260.0 \mathrm{n}$ ad $=2.3 \mathrm{e}-13 \mathrm{as}=2.3 \mathrm{e}-13 \mathrm{pd}=2.46 \mathrm{u} \mathrm{ps}=2.46 \mathrm{u} \mathrm{nrd}=0.13$ $\mathrm{nrs}=0.13 \mathrm{sa}=230.0 \mathrm{n} \mathrm{sb}=230.0 \mathrm{n}$ sca=0 $\mathrm{scb}=0 \mathrm{scc}=0$ mismatchflag=1 sigma $=1$

$\mathrm{M} 2 \backslash\langle 27\rangle>$ (distance enb $\backslash<27 \backslash>A$ vdd) pch_hvt_mac I=1u w=1u multi=(1) $n f=1$ $\mathrm{sd}=260.0 \mathrm{n}$ ad $=2.3 \mathrm{e}-13$ as $=2.3 \mathrm{e}-13 \mathrm{pd}=2.46 \mathrm{u}$ ps $=2.46 \mathrm{u} \mathrm{nrd}=0.13$ $\mathrm{nrs}=0.13 \mathrm{sa}=230.0 \mathrm{n} \mathrm{sb}=230.0 \mathrm{n} \mathrm{sca}=0 \mathrm{scb}=0 \mathrm{scc}=0$ mismatchflag=1 sigma $=1$

$\mathrm{M} 2 \backslash<28 \backslash>$ (distance enb $\backslash<28 \backslash>\mathrm{A}$ vdd) pch_hvt_mac $\mathrm{I}=1 \mathrm{u}$ w=1u multi=(1) $\mathrm{nf}=1$ $\mathrm{sd}=260.0 \mathrm{n}$ ad $=2.3 \mathrm{e}-13$ as $=2.3 \mathrm{e}-13 \mathrm{pd}=2.46 \mathrm{u}$ ps $=2.46 \mathrm{u} \mathrm{nrd}=0.13$ । $\mathrm{nrs}=0.13$ sa $=230.0 \mathrm{n} \quad \mathrm{sb}=230.0 \mathrm{n} \quad \mathrm{sca}=0 \quad \mathrm{scb}=0 \quad \mathrm{scc}=0$ mismatchflag $=1$ । $\mathrm{nrs}=0.13$
$\mathrm{sigma}=1$

$\mathrm{M} 2 \backslash<29 \backslash>$ (distance enb $\backslash<29 \backslash>A$ vdd) pch_hvt_mac $\mathrm{I}=1 \mathrm{u} \mathrm{w}=1 \mathrm{u}$ multi $=(1) \mathrm{nf}=1\rangle$ $\mathrm{sd}=260.0 \mathrm{n}$ ad $=2.3 \mathrm{e}-13$ as $=2.3 \mathrm{e}-13 \mathrm{pd}=2.46 \mathrm{u}$ ps $=2.46 \mathrm{u} \mathrm{nrd}=0.13$ $\mathrm{nrs}=0.13 \mathrm{sa}=230.0 \mathrm{n} \mathrm{sb}=230$. 0 n sca=0 $\mathrm{scb}=0 \mathrm{scc}=0$ mismatchflag=1 sigma $=1$

$\mathrm{M} 2 \backslash<30 \backslash>($ distance enb $\backslash<30 \backslash>\mathrm{A}$ vdd) pch_hvt_mac $\mathrm{I}=1 \mathrm{u}$ w=1u multi=(1) $n f=1 \backslash$ 
$\mathrm{sd}=260.0 \mathrm{n}$ ad $=2.3 \mathrm{e}-13$ as $=2.3 \mathrm{e}-13 \mathrm{pd}=2.46 \mathrm{u} \mathrm{ps}=2.46 \mathrm{u} \mathrm{nrd}=0.13$ $\mathrm{nrs}=0.13 \mathrm{sa}=230.0 \mathrm{n} \mathrm{sb}=230.0 \mathrm{n} \quad \mathrm{sca}=0 \mathrm{scb}=0 \mathrm{scc}=0$ mismatchflag=1 \} sigma $=1$

$\mid 72 \backslash<1 \backslash>$ (pulse_out $\backslash<1 \backslash>$ window en $\backslash<1 \backslash>$ vss vdd) manhattan_xor $172 \backslash<2\rangle$ (pulse_out $|<2\rangle>$ window en $|<2\rangle>$ vss vdd) manhattan_xor $172 \backslash<3\rangle>$ (pulse_out $\langle<3\rangle>$ window en $|<3\rangle>$ vss vdd) manhattan_xor $172 \backslash<4 \backslash>$ (pulse_out $\langle<4\rangle$ window en $\mid<4 \backslash>$ vss vdd) manhattan_xor $172\langle<5\rangle>$ (pulse out $\mid<5 \backslash>$ window en $\mid<5 \backslash>$ vss vdd) manhattan_xor $172\langle<6\rangle>$ (pulse_out $|<6\rangle>$ window en $|<6|>$ vss vdd) manhattan_xor $172\langle<6>>$ (pulse_out $|<6 \backslash>$ window en $|<6|>$ vss vdd) manhattan_xor
$172\langle<\rangle>$ (pulse_out $\langle<7>$ window en $|<7 \backslash>$ vss vdd) manhattan_xor $172|<8|>$ (pulse_out $|<8|>$ window en $|<8|>$ vss vdd) manhattan_xor $172 \backslash<9 \mid>$ (pulse_out $\backslash<9 \mid>$ window en $\backslash<9 \backslash>$ vss vdd) manhattan_xor $172 \backslash<10 \backslash>$ (pulse_out $\backslash<10 \backslash>$ window en $\backslash<10 \backslash>$ vss vdd) manhattan_xor $172 \backslash<11 \backslash>$ (pulse_out $\backslash<11 \backslash>$ window en $\backslash<11 \backslash>$ vss vdd) manhattan_xor $|72 \backslash<12|>$ (pulse_out $\backslash<12 \mid>$ window en $\backslash<12\rangle>$ vss vdd) manhattan _xor $172 \backslash<13 \backslash>$ (pulse_out $\backslash<13 \backslash>$ window en $\backslash<13 \backslash>$ vss vdd) manhattan_xor $172 \backslash<14 \mid>$ (pulse_out $\backslash<14 \backslash>$ window en $\backslash<14 \mid>$ vss vdd) manhattan_xor $172 \backslash<15 \backslash>$ (pulse_out $\backslash<15 \backslash>$ window en $\backslash<15 \backslash>$ vss vdd) manhattan_xor $\mid 72\langle<16\rangle>$ (pulse_out $\langle<16\rangle>$ window en $\backslash<16 \mid>$ vss vdd) manhattan_xor $172 \backslash<17\rangle>$ (pulse_out $\backslash<17 \backslash>$ window en $\backslash<17 \backslash>$ vss vdd) manhattan_xor $172<<19 \mid>$ (pulse 0 (1) $|<19|>$ window en $<19 \mid>$ vss vdd) manhattan $x$ or

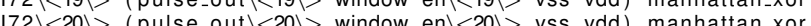
172) $<21>$ (pulse_out $<20>$ window en $<201>$ vss vdd) manhattan_xor $172\langle<21\rangle$ (pulse _out $\langle<21\rangle$ window en $\mid<21 \backslash>$ vss vdd) manhattan_xor $172|<22|>$ (pulse_out $|<22|>$ window en $|<22|>$ vss vdd) manhattan_xor $172|<23|>$ (pulse_out $|<23|>$ window en $|<23|>$ vss vdd) manhattan_xor $172 \backslash<24 \backslash>$ (pulse_out $\backslash<24 \backslash>$ window en $\backslash<24 \backslash>$ vss vdd) manhattan_xor $172 \backslash<25 \backslash>$ (pulse_out $\backslash<25 \backslash>$ window en $\backslash<25 \backslash>$ vss vdd) manhattan_xor $172 \backslash<26 \mid>$ (pulse_out $\backslash<26 \backslash>$ window en $\backslash<26 \backslash>$ vss vdd) manhattan_xor $172 \backslash<27 \backslash>$ (pulse_out $\backslash<27 \backslash>$ window en $\backslash<27 \backslash>$ vss vdd) manhattan_xor $172 \backslash<28 \backslash>$ (pulse_out $\backslash<28 \backslash>$ window en $\backslash<28 \backslash>$ vss vdd) manhattan_xor $172 \backslash<29 \backslash>$ (pulse_out $\backslash<29 \backslash>$ window en $\backslash<29 \backslash>$ vss vdd) manhattan_xor $172 \backslash<30 \backslash>$ (pulse_out $\backslash<30 \backslash>$ window en $\backslash<30 \backslash>$ vss vdd) manhattan_xor 


\section{Referências}

ABBOTT, A. In search of the sixth sense. Nature, v. 442, n. 13, p. 125-127, Jul 2006.

ABDELHALIM, K.; KOKAROVTSEVA, L.; VELAZQUEZ, J. L. P.; GENOV, R. 915-MHz FSK/OOK Wireless Neural Recording SoC With 64 Mixed-Signal FIR Filters. IEEE Journal of Solid-State Circuits, v. 48, n. 10, p. 2478-2493, Oct. 2013.

AFLALO, T.; KELLIS, S.; KLAES, C.; LEE, B.; SHI, Y.; PEJSA, K.; SHANFIELD, K.; HAYES-JACKSON, S.; AISEN, M.; HECK, C.; LIU, C.; ANDERSEN, R. A. Decoding motor imagery from the posterior parietal cortex of a tetraplegic human. Science, v. 348, n. 6237, p. 906-910, may. 2015.

AKIN, T.; NAJAFI, K.; BRADLEY, R. M. A Wireless Implantable Multichannel Digital Neural Recording System for a Micromachined Sieve Electrode. IEEE Journal of Solid-State Circuits, v. 33, n. 1, p. 109-118, Jan. 1998.

BERGER, T. W.; SONG, D.; CHAN, R. H. M.; MARMARELIS, V. Z.; LACOSS, J.; WILLS, J.; HAMPSON, R. E.; DEADWYLER, S. A.; GRANACKI, J. J. A Hippocampal Cognitive Prosthesis: Multi-Input, Multi-Output Nonlinear Modeling and VLSI Implementation. IEEE Transactions on Neural Systems and Rehabilitation Engineering, v. 20, p. 198-211, mar. 2012.

BLUM, R. A.; ROSS, J. D.; BROWN, E. A.; DEWEERTH, S. P. An integrated system for simultaneous, multichannel neural stimulation and recording. IEEE Transactions on Circuits ans Systems I: Fundamental Theory and Applications, v. 54, n. 12, p. 2608-2618, Dec. 2007.

BROWN, E. A.; ROSS, J. D.; BLUM, R. A.; NAM, Y.; WHEELER, B. C.; DEWEERTH, S. P. Stimulus-artifact elimination in a multi-electrode system. IEEE Transactions on Biomedical Circuits and Systems, v. 2, n. 1, p. 10-21, Mar. 2008.

CHAE, M.; LIU, W.; YANG, Z.; CHEN, T.; KIM, J.; SIVAPRAKASAM, M.; YUCE, M. A 128-Channel $6 \mathrm{~mW}$ wireless neural recording IC with on-the-fly spike sorting and UWB transmitter. In: IEEE INTERNATIONAL SOLID-STATE CIRCUITS CONFERENCE. San Francisco, 2008. p. 145-147.

CHAE, M. S.; LIU, W.; SIVAPRAKASAM, M. Design Optimization for Integrated Neural Recording Systems. IEEE Journal of Solid-State Circuits, v. 43, n. 9, p. 123-133, Sep. 2008.

CHAE, M. S.; YANG, Z.; YUCE, M. R.; HOANG, L.; LIU, W. A 128-Channel 6 mW Wireless Neural Recording IC With Spike Feature Extraction and UWB Transmitter. IEEE Transactions on Neural Systems and Rehabilitation Engineering, v. 17, p. 312-321, aug. 2009. 
CHOI, J. H.; JUNG, H. K.; KIM, T. A New Action Potential Detector Using MTEO and Its Effects on Spike Sorting Systems at Low Signal-to-Noise Ratios. IEEE Transactions on Biomedical Engineering, v. 53, n. 4, p. 738-746, Apr. 2006.

COLLINGER, J. L.; WODLINGER, B.; DOWNEY, J.; WANG, W.; TYLER-KABARA, E. C.; WEBER, D. J.; MCMORLAND, A. J. C.; VELLISTE, M.; BONINGER, M. L.; SCHWARTZ, A. B. High-performance neuroprosthetic control by an individual with tetraplegia. Lancet, v. 381, p. 557-564, feb. 2013.

DATTA-CHAUDHURI, T.; SENEVIRATHNA, B.; SMELA, A. C. ans E.; ABSHIRE, P. An active micro-electrode array with spike detection and asynchronous readout. In: IEEE BIOMEDICAL CIRCUITS AND SYSTEMS CONFERENCE. Lausanne, 2014. p. 588-591.

DEJEMOUAI, A.; SAWAN, M.; SLAMANI, M. A 200 MHz Frequency-Locked loop based on new frequency-to-voltage converters approach. In: IEEE INTERNATIONAL SYMPOSIUM ON CIRCUITS AND SYSTEM. Orlando, Florida, 1999. p. 89-92.

DHANASEKARAN, V.; GAMBHIR, M.; ELSAYED, M. M.; SANCHEZ-SINENCIO, E.; SILVA-MARTINEZ, J.; MISHRA, C.; CHEN, L.; PANKRATZ, E. J. A continuous time multi-bit $\triangle \Sigma$ ADC using time domain quantizer and feedback element. IEEE Journal of Solid-State Circuits, v. 46, n. 3, p. 639-650, Mar. 2011.

DO, A. T.; YEO, K. S. A hybrid NEO-based spike detection algorithm for implantable brain-IC interface applications. In: IEEE INTERNATIONAL SYMPOSIUM ON CIRCUITS AND SYSTEM. Melbourne, 2014. p. 2393-2396.

DRAGAS, J.; JACKEL, D.; HIERLEMANN, A.; FRANKE, F. Complexity Optimization and High-Throughput Low-Latency Hardware Implementation of a Multi-Electrode Spike-Sorting Algorithm. IEEE Transactions on Neural Systems and Rehabilitation Engineering, v. 23, p. 149-158, Mar 2015.

FETZ, E. Operant conditioning of cortical unit activity. Science, v. 28, n. 163, p. 955-958, Feb 1969.

GAO, H.; WALKER, R. M.; NUYUJUKIAN, P.; MAKINWA, K.; SHENOY, K. V.; MENG, B. M. ans T. H. Hermese: A 96-channel full data rate direct neural interface in $0.13 \mu \mathrm{m}$ cmos. IEEE Journal of Solid-Stat Circuits, v. 47, n. 4, p. 1043-1055, Abr. 2012.

GIBSON, S. Technology-Aware Algorithm Design for Neural Spike Detection, Feature Extraction, and Dimensionality Reduction. IEEE Transactions on Neural Systems and Rehabilitation Engineering, v. 18, n. 5, p. 469-478, 2010.

GIBSON, S.; JUDY, J. W.; MARKOVIĆ, D. Comparison of spike-sorting algorithms for future hardware implementation. In: 30TH ANNUAL INTERNATIONAL IEEE EMBS CONFERENCE, 2008, Vancouver. Proceedings... Vancouver: IEEE, 2008. p. 5015-5020.

GOSSELIN, B.; SAWAN, M. An Ultra Low-Power CMOS Automatic Action Potential Detector. IEEE Transactions on Neural Systems and Rehabilitation Engineering, v. 17, n. 4 , p. 346-353, 2009. 
GRAIMANN, B.; ALLISON, B.; PFURTSCHELLER, G. Brain-computer interfaces: a gentle introduction. In: GRAIMANN, B.; ALLISON, B.; PFURTSCHELLER, G. (Ed.). Berlin: Springer, 2010. cap. Brain-computer interfaces, p. 1-27.

GUTTMAN, M. M.; ROBERTS, G. W. Sampled-Data IIr Filtering Using Time-Mode Signal Processing Circuits. In: IEEE INTERNATIONAL SYMPOSIUM ON CIRCUITS AND SYSTEM. Taipei, 2009. p. 2285-2288.

HARRIS, K. D.; HENZE, D. A.; CSICSVARI, J.; HIRASE, H.; BUZSÁKI, G. Accuracy of tetrode spikes separation as determined by intracelullar and extracellular measurements. Journal of Neurophysiology, v. 84, n. 1, p. 401-414, Jul. 2000.

HARRISON, R.; WATKINS, P.; KIER, R.; LOVEJOY, R.; BLACK, D.; NORMANN, R.; SOLZBACHER, F. A Low-Power Integrated Circuit for a Wireless 100-Electrode Neural Recording System. In: IEEE INTERNATIONAL SOLID-STATE CIRCUITS CONFERENCE. San Francisco, 2006. p. 2258-2267.

HARRISON, R. R. A Low-Power Integrated Circuit for Adaptive Detection of Action Potentials in Noisy Signals. In: $25^{\text {th }}$ ANNUAL INTERNATIONAL CONFERENCE OF THE IEEE EMBS, 2003, Cancun. Proceedings... Cancun: IEEE, 2003. p. 3325-3328.

HARRISON, R. R.; CHARLES, C. A Low-Power Low-Noise CMOS Amplifier for Neural Recording Applications. IEEE Journal of Solid-State Circuits, v. 38, n. 6, p. 958-965, Jun. 2003.

HE, B. Neural Engineering. 2. ed. New York: Springer New York, 2013.

HENZE, D. A.; BORTHEGYI, Z.; CSICSVARI, J.; MAMIYA, A.; HARRIS, K. D.; BUZSÁKI, G. Intracellular features predicted by extracellular recordings in the hippocampus in vivo. Journal of Neurophysiology, v. 84, n. 1, p. 390-400, Jul. 2000.

HISENI, S.; SAWIGUN, C.; NGAMKHAM, W.; SERDIJN, W. A. A Compact, Nano-Power CMOS Action Potential Detector. In: IEEE BIOMEDICAL CIRCUITS AND SYSTEMS CONFERENCE. Beijing, China, 2009. p. 97-100.

HOCHBERG, L. R.; BACHER, D.; JAROSIEWICZ, B.; MASSE, N. Y.; SIMERAL, J. D.; VOGEL, J.; HADDADIN, S.; LIU, J.; CASH, S. S.; SMAGT, P. van der; DONOGHUE, J. P. Reach and grasp by people with tetraplegia using a neurally controlled robotic arm. Nature, v. 485, p. 372-377, may. 2012.

HOCHBERG, L. R.; SERRUYA, M. D.; FRIEHS, G. M.; MUKAND, J. A.; SALEH, M.; CAPLAN, A. H.; BRANNER, A.; CHEN, D.; PENN, R. D.; DONOGHUE, J. P. Neuronal ensemble control of prosthetic devices by a human with tetraplegia. Nature, v. 442, p. 164-171, jul. 2006.

HOLLEMAN, J.; MISHRA, A.; DIORIO, C.; OTIS, B. A Micro-Power Neural Spike Detector and Feature Extractor in $0.13 \mu \mathrm{m}$ CMOS. In: IEEE CUSTOM INTEGRATED CIRCUITS CONFERENCE. San Jose, CA, 2008. p. 333-336.

HOUSE, W.; URBAN, J. Long term results of electrode implantation and electronic stimulation of the cochlea in man. Annals of Otology, Rhinology and Laryngology, v. 82, p. 504-715, jul-aug 1973. 
IFFT, P. J.; SHOKUR, S.; LI, Z.; LEBEDEV, M. A.; NICOLELIS, M. A. L. A Brain-Machine Interface Enables Bimanual Arm Movements in Monkeys. Science Translation Medicine, v. 5, n. 210, p. 1-15, 2013.

JACKSON, A. Brain-controlled robot grabs attention. Nature, v. 485, p. 317-318, may. 2012.

KAISER, J. F. On a simple algorithm to calculate the energy of a signal. In: INTERNATIONAL CONFERENCE OF ACOUSTIC, SPEECH AND SIGNAL PROCESSING, 1990, Albuquerque. Proceedings... New Mexico: IEEE, 1990. p. 381-384.

KAISER, J. F. On Teager's Energy Algorithm and Its Generalization to Continuous Signals. In: 4TH IEEE DSP WORKSHOP, 1990, Monhonk, NY. Proceedings... [S.I.]: IEEE, 1990.

KARKARE, V.; GIBSON, S.; MARKOVIC, D. A 75- $\mu \mathrm{w}, 16$-channel neural spike-sorting processor with unsupervised clustering. IEEE Journal of Solid-State Circuits, v. 48, p. 2230-2238, Sep. 2013.

KIM, S.; TATHIREDDY, P.; NORMANN, R. A.; SOLZBACHER, F. Thermal impact of an active 3-D microelectrode array implanted in the brain. IEEE Transactions on Neural Systems and Rehabilitation Engineering, v. 15, n. 4, p. 493-501, Dez. 2007.

KOUTSOS, E.; PARASKEVOPOULOU, S.; CONSTANDINOU, T. A 1.5 uW NEO-baed Spike Detector with Adaptive-Threshold for Calibration-free Multichannel Neural Interfaces. In: IEEE INTERNATIONAL SYMPOSIUM ON CIRCUITS AND SYSTEM. Beijing, 2013. p. 1922-1925.

KWOK, R. Once more, with feeling. Nature, v. 497, p. 176-178, may. 2013.

LAPOLLI Ângelo C.; COPPA, B.; HÉLIOT, R. Low-Power Hardware for Neural Spike Compression in BMIs. In: $35^{\text {th }}$ ANNUAL INTERNATIONAL CONFERENCE OF THE IEEE EMBS, 2013, Osaka, Japan. Proceedings... Osaka, Japan: IEEE, 2013. p. 2156-2159.

LI, H.; XU, Q. Sub-threshold-based ultra-low-power neural spike detector. Electronics Letters, v. 47, n. 6, p. 1-2, Mar. 2011.

LI, Y.-G.; MA, Q.; HAIDER, M. R.; MASSOUD, Y. Ultra-Low-Power High Sensitivity Spike Detectors Based on Modified Nonlinear Energy Operator. In: IEEE INTERNATIONAL SYMPOSIUM ON CIRCUITS AND SYSTEM. Beijing, 2013. p. 137-140.

LIU, S.; KRAMER, J.; INDIVERI, G.; DELBRüCK, T.; DOUGLAS, R. Analog VLSI: Circuits ans Principles. 1. ed. Massachusetts: Massachusetts Institute of Technology, 2002.

LÓPEZ-MARTÍN, A. J.; ESPARZA-ALFARO, F.; RAMIREZ-ANGULO, J.; CARVAJAL, R. G. Accurate micropower class ab cmos voltage-to-current converter. In: 20TH EUROPEAN CONFERENCE ON CIRCUIT THEORY AND DESIGN (ECCTD), 2011, Linkoping. Proceedings... Linkoping: IEEE, 2011. p. 114-117. 
MEAD, C. Analog VLSI and Neural Systems. 1. ed. Reading,MA: Addison Wesley, 1989.

MESTAIS, C.; CHAVERT, G.; SAUTER-STARACE, F.; FOESTER, M.; RATEL, D.; BENABID, A. L. WIMAGINE: Wireless 64-channel ECoG recording implant for long term clinical applications. IEEE Transactions on Neural Systems and Rehabilitation Engineering, v. 23, n. 1, p. 10-21, Jan. 2015.

MOHAMAD, S.; TANG, F.; BERMAK, A.; AMIRA, A.; BENAMMAR, M. A Low Power Temperature Sensor based on a Voltage to Time Converter Cell. In: 25TH INTERNATIONAL CONFERENCE ON MICROELECTRONICS. Beirut, 2013. p. 1-4.

MOHSENO, P.; NAJAFI, K. A Fully Integrated Neural Recording Amplifier With DC Input Stabilization. IEEE Transactions on Biomedical Engineering, v. 51, n. 5, p. 832-837, May. 2004.

MONGE, M.; RAJ, M.; HONAVAR-NAZARI, M.; CHANG, H.-C.; ZHAO, Y.; WEILAND, J.; HUMAYUN, M.; TAI, Y.-C.; EMAMI-NEYESTANAK, A. A Fully Intraocular $0.0169 \mathrm{~mm}^{2} /$ pixel $512-C h a n n e l$ Self-Calibrating Epiretinal Prosthesis in 65nm CMOS. In: IEEE INTERNATIONAL SOLID-STATE CIRCUITS CONFERENCE. San Francisco, 2013. p. 296-297.

MUKHOPADHYAY, S.; RAY, G. C. A new interpretation of nonlinear energy operator and its efficacy in spike detection. IEEE Transactions on Biomedical Circuits and Systems, v. 45, n. 2, p. 180-187, Feb. 1998.

MULLER, R.; LE, H.-P.; LI, W.; LEDOCHOWITSCH, P.; GAMBINI, S.; BJORNINEN, T.; KORALEK, A.; CARMENA, J. M.; MAHABARBIZ, M. M.; ALON, E.; RABAEY, J. M. A Miniaturized 64-Channel 225uW Wireless Electrocorticographic Neural Sensor. In: IEEE INTERNATIONAL SOLID-STATE CIRCUITS CONFERENCE. San Francisco, 2014. p. 412-414.

MULLER, R.; LE, H.-P.; LIM, W.; LEDOCHOWITSCH, P.; GAMBINI, S.; BJORNINEN, T.; KORALEK, A.; CARMENA, J. M.; MAHARIZ, M. M.; ALON, E.; RABAEY, J. M. A Minimally Invansive 64-Channel Wireless $\mu$ ECoG Implant. IEEE Journal of Solid-State Circuits, v. 50, n. 1, p. 344-359, Jan. 2015.

NADEAU, P. M.; PAIDIMARRI, A.; CHANDRAKASAN, A. P. Ultra Low-Energy Relaxation Oscillator With $230 \mathrm{fJ} /$ cycle Efficiency. IEEE Journal of Solid-State Circuits, v. 51, n. 4, p. 798-808, Apr. 2016.

NAJAFI, K.; WISE, K. A High-Yield IC-Compatible Multichannel Recording Array. IEEE Transactions on Electron Devices, ED-32, n. 7, p. 1206-1211, Jul. 1985.

NAVAJAS, J.; BARSAKCIOGLU, D. Y.; EFTEKHAR, A.; JACKSON, A.; CONSTANDINOU, T. G.; QUIROGA, R. Q. Minimum requirements for accurate and efficient real-time on-chip spike sorting. Journal of Neuroscience Methods, v. 230, p. 51-64, Jun 2014.

NG, K. A.; XU, Y. P. A Multi-Channel Neural-Recording Amplifier System with 90dB CMRR EMploying CMOS-Inverter-Baased OTAs with CMFB Through SUpply Rails in 
65nm CMOS. In: IEEE INTERNATIONAL SOLID-STATE CIRCUITS CONFERENCE. San Francisco, 2015. p. 205-207.

OBEID, I. Comparison of spike detectors based on simultaneous intracellular and extracellular recordings. In: 3RD INTERNATIONAL IEEE EMBS CONFERENCE ON NEURAL ENGINEERING, 2007, Kohala Coast, HI. Proceedings... USA: IEEE, 2007. p. 410-413.

OLSSON-III, R. H.; WISE, K. D. A three-dimensional neural recording microsystem with implantable data compression circuitry. IEEE Journal of Solid-State Circuits, v. 40 , n. 12, p. 2796-2804, Dec. 2005.

PARASKEVOPOULOU, S. E.; BARSAKCIOGLU, D. Y.; SABERI, M. R. Feature extraction using first and second derivative extrema (fsde) for real-time and hardwareefficient spike sorting. Journal of Neuroscience Methods, v. 215, n. 1, p. 29-37, Apr 2013.

PEKAU, H.; YOUSIF, A.; HASLETT, J. W. A CMOS Integrated Linear VOltage-toPulse-Delay-Time Converter for Time Based Analog-to-Digital Converters. In: IEEE INTERNATIONAL SYMPOSIUM ON CIRCUITS AND SYSTEM. Island of Kos, 2006. p. 2373-2376.

QUIROGA, R. Q.; NADASDY, Z.; BEN-SHAUL, Y. Unsupervised Spike Detection and Sorting with Wavelets and Superparamagnetic Clustering. Neural Computation, v. 16, n. 8, aug 2004.

RAVINUTHULA, V.; GARG, V.; HARRIS, J. G.; FORTES, J. A. B. Time-mode circuits for analog computation. International Journal of Circuit Theory and Applications, v. 37, n. 5, p. 631-659, Jun. 2009.

RAZAVI, B. Design of Analog CMOS Integrated Circuits. 1. ed. New York, NY: McGraw-Hill, 2001.

RIZK, M.; WOLF, P. D. Optimizing the Automatic Selection of Spike Detection Thresholds Using a Multiple of the Noise Level. Medical and biological engineering and computing, v. 47, n. 9, p. 955-966, Sep 2009.

RODRÍGUEZ-PÉREZ, A.; RUIZ-AMAYA, J.; DELGADO-RESTITUTO, M.; RODRÍGUEZ-VÁZQUEZ Ángel. A low-power programmable neural spike detection channel with embedded calibration and data compression. IEEE Transactions on Biomedical Circuits and Systems, v. 6, n. 2, p. 87-100, Apr. 2012.

RUTISHAUSER, U.; SCHUMAN, E. M.; MAMELAK, A. N. Online detection and sorting of extracellularly recorded action potentials in human medial temporal lobe recordings, in vivo. Journal of Neuroscience Methods, v. 154, p. 204-224, apr 2006.

SALAM, M. T.; NGUYEN, D. K.; SAWAN, M. A Low-power Implantable Device for Epileptic Seizure Detection and Neurostimulation. In: IEEE BIOMEDICAL CIRCUITS AND SYSTEMS CONFERENCE. Paphos, 2010. p. 154-157. 
SALDAÑA-PUMARICA, J.; DEL-MORAL-HERNANDEZ, E. A CMOS Implementation of the Discrete Time Nonlinear Energy Operator Based on a Transconductor-Squarer Circuit. In: IEEE LATIN AMERICAN SYMPOSIUM ON CIRCUITS AND SYSTEM (LASCAS). Florianopolis, SC, Brazil, 2016.

SALDAÑA-PUMARICA, J.; DEL-MORAL-HERNANDEZ, E. A CMOS Implementation of the Discrete Time Nonlinear Energy Operator Based on Current Squarer Circuit. In: IBERCHIP. Florianopolis, SC, Brazil, 2016.

SANTHANAM, G.; LINDERMAN, M. D.; GILJA, V.; AFSHAR, A.; RYU, S. I.; MENG, T.; SHENOY, K. HermesB: A continuous neural recording systems for freely behaving primates. IEEE Transactions on Biomedical Engineering, v. 54, n. 11, p. 2037-2050, Nov. 2007.

SCHALK, G. Can Electrocorticography (ECoG) support robust and powerful brain-computer interfaces? Frontiers in Neuroengineering, v. 3, n. 9, Jun. 2010.

SCHWARTZ, D. A.; LEBEDEV, M. A.; HANSON, T. L.; DIMITROV, D. F.; LEHEW, G.; MELOY, J.; RAJANGAM, S.; SUBRAMANIAN, V.; IFFT, P. J.; LI, Z.; RAMAKRISHNA, A.; TATE, A.; ZHUANG, K. Z.; NICOLELIS, M. A. L. Chronic, wireless recordings of large-scale brain activity in freely moving rhesus monkeys. Nature Methods, v. 11, p. 670-679, Jun. 2014.

SODAGAR, A. M.; PERLIN, G. E.; YAO, Y.; WISE, K. D.; NAJAFI, K. An Implantable Microsystem for Wireless Multi-Channel Cortical Recording. In: SOLID-STATE SENSORS, ACTUATORS AND MICROSYSTEMS CONFERENCE, 2007, Lyon. Proceedings... Antalya: IEEE, 2007. p. 69-72.

SODAGAR, A. M.; PERLIN, G. E.; YAO, Y.; NAJAFI, K.; WISE, K. D. An implantable 64-channel wireless microsystem for single-unit neural recording. IEEE Journal of Solid-State Circuits, v. 44, n. 9, p. 2591-2604, Sep. 2009.

SODAGAR, A. M.; WISE, K. D.; NAJAFI, K. A Fully-Integrated Mixed-Signal Neural Processing Module for IMplantable Multi-Channel Cortical Recording. In: IEEE BIOMEDICAL CIRCUITS AND SYSTEMS CONFERENCE. London, 2006. p. 37-40.

TAILLEFER, C. S.; ROBERTS, G. W. Delta-Sigma A/D conversion via time-mode signal processing. IEEE Transactions on Circuits and Systems-I: Regular Papers, v. 56, n. 9, p. 1908-1920, Set. 2009.

TAO, S.; RUSU, A. A Power-Efficient Continuous-Time Incremental Sigma-Delta ADC for Neural Recording Systems. IEEE Transactions on Circuits and Systems-I:

Regular Papers, v. 62, n. 6, p. 1489-1498, Jun. 2015.

TEAGER, H. M.; TEAGER, S. M. Speech production and speech modelling. In: HARDCASTLE, W. J.; MARCHAL, E. A. (Ed.). France: NATO Advanced Study Inst. Series D, 1989. v. 55, cap. Evidence for Nonlinear Sound Production Mechanism in the Vocal Tract.

TSIVIDIS, Y. Operation and Modeling of THE MOS Transitor. 2. ed. Oxford New York: Oxford University Press, 1999. 
TSIVIDIS, Y.; MCANDREW, C. Operation and Modeling of THE MOS Transitor. 3. ed. Oxford New York: Oxford University Press, 2011.

UNNIKRISHNAN, V.; VESTERBACKA, M. Time-Mode Analog-to-Digital Conversion Using Standard Cells. IEEE Transactions on Circuits and Systems-I: Regular Papers, v. 61, n. 12, p. 3348-3357, Dez. 2014.

VIGRAHAM, B.; KUPPAMBATTI, J.; KINGET, P. R. Switched-Mode Operational Amplifiers and Their Application to Continuous-Time Filters in Nanoscale CMOS. IEEE Journal of Solid-Stat Circuits, v. 49, n. 12, p. 2758-2772, Dez. 2014.

WISE, K. D.; ANGELL, J. B. A Low-Cpacitance Multielectrode Probe for Use in Extracellular Neurophysiology. IEEE Transactions on Biomedical Engineering, BME-22, n. 3, p. 212-219, May. 1975.

WOOD, F.; BLACK, M. J.; VARGAS-IRWIN, C.; FELLOWS, M.; DONOGHUE, J. P. On the Variability of Manual Spike Sorting. IEEE Transactions on Biomedical Engineering, v. 51, n. 6, p. 912-918, 2004.

YANG, Y.; MASON, A. J. Optimization of nonlinear energy operator based spike detection circuit for high density neural recordings. In: IEEE INTERNATIONAL SYMPOSIUM ON CIRCUITS AND SYSTEMS. Melbourne, 2014. p. 1396-1399.

ZAMANI, M.; DEMOSTHENOUS, A. Feature Extraction Using Extrema Sampling of Discrete Derivatives for Spike Sorting in Implantable Upper-Limb Neural Prostheses. IEEE Transactions on Neural Systems and Rehabilitation Engineering, v. 22, p. 716-726, Jul 2014.

ZEINOLABEDIN, S. M. A.; DO, A. T.; YEO, K. S.; KIM, T.-H. Design of an hybrid neural spike detection algorithm for implantable integrated brain circuits. In: IEEE INTERNATIONAL SYMPOSIUM ON CIRCUITS AND SYSTEM. Lisbon, Portugal, 2015. p. 794-797.

ZHENG, L.; LEENE, L. B.; LIU, Y.; CONSTANDINOU, T. G. An Adaptive 16/64kHz, 9-bit SAR ADC with Peak-Aligned Sampling for Neural Spike Recording. In: IEEE INTERNATIONAL SYMPOSIUM ON CIRCUITS AND SYSTEMS. Melbourne, 2014. p. 2385-2388.

ZHU, G.; YUAN, F.; KHAN, G. Time-Mode Approach for Mixed Analog-Digital Signal Processing. Journal of Electrical \& Electronic Systems, v. 2, n. 1, p. 1-4, jan 2013.

ZOU, X.; LIU, L.; CHEONG, J. H.; YAO, L.; LI, P.; CHENG, M.-Y.; GOH, W. L.; RAJKUMAR, R.; DAWE, G. S.; CHENG, K.-W.; JE, M. A 100-channel 1-mw implantable neural recording ic. IEEE Transactions on Circuits and Systems, v. 60, n. 10, p. 2584-2596, Out. 2013.

ZVIAGINTSEV, A.; PERELMAN, Y.; GINOSAR, R. Algoeithms and architectures for low power spike detection and alignment. Journal of Neural Engineering, v. 3, p. 35-42, Jan. 2006. 